\title{
Directional Wavenumber Characteristics of Short Sea Waves
}

\author{
by \\ Nicole Marie Suoja \\ B.S., Mechanical Engineering, Washington State University (1994) \\ M.S., Ocean Engineering, Massachusetts Institute of Technology (1996) \\ Submitted in partial fulfillment of the \\ requirements for the degree of \\ DOCTOR OF PHILOSOPHY IN OCEANOGRAPHIC ENGINEERING \\ at the \\ MASSACHUSETTS INSTITUTE OF TECHNOLOGY \\ and the \\ WOODS HOLE OCEANOGRAPHIC INSTITUTION
}

February 2000

(C) 2000 Nicole Marie Suoja

All rights reserved.

The author hereby grants to MIT and WHOI permission to reproduce paper and electronic copies of this thesis in whole or in part and to distribute them publicly.

Signature of Author

Joint Program in Applied Ocean(Sciende)and Engineering

Massachusetts Institute of Technology (MIT)

Woods Hole Oceanographic Institution (WHOI)

Certified by

Dr. Jerome Milgram

Professor, Department of Ocean Engineering, MIT

Thesis supervisor

Certifiel by

Dr. Erik J. Bock

Interdiscipliplinary Center for Scientific Computing, University of Heidelberg

Thesis supervisor

Accepted by

Dr. Michael Triantafyllou

Professor, Department of Ocean Engineering, MIT

Chairman, Joint Committee for Applied Ocean Science and Engineering, MIT/WHOI 


\title{
Directional Wavenumber Characteristics of Short Sea Waves
}

\author{
by \\ Nicole Marie Suoja \\ Submitted February, 2000 in partial fulfillment of the \\ requirements for the degree of \\ DOCTOR OF PHILOSOPHY IN OCEANOGRAPHIC ENGINEERING
}

\begin{abstract}
Interest in short waves on the ocean surface has been growing over the last three decades because they play an important role in surface electromagnetic (e.m.) scattering. Currently radars and scatterometers which use e.m. scattering to remotely examine the ocean can produce estimates of the surface wind field, surface currents, and other scientifically important ocean processes. These estimates are based on models which depend on a thorough understanding of electromagnetic scattering mechanisms, and of the three-dimensional surface wave field. Electromagnetic scattering theory is well developed, but the short wavelength portion of the surface wave field has only recently been experimentally explored. A single, consistent, and accurate model of the energy distribution on the ocean surface, also known as the wave height spectrum, has yet to be developed.

A new instrument was developed to measure the height of waves with $2-30 \mathrm{~cm}$ wavelengths at an array of locations which can be post-processed to generate an estimate of the two-dimensional wave height spectrum. This instrument (a circular wire wave gage buoy) was deployed in an experiment which gathered not only in situ measurements of the two-dimensional wave height spectrum, but also coincident scatterometer measurements, allowing the comparison of current e.m. scattering and surface wave height spectrum models with at sea data,

The experiment was conducted at the Buzzards Bay Tower located at the mouth of Buzzards Bay in Massachusetts. A rotating X-band scatterometer, a sonic anemometer, and a capacitive wire wave gage were mounted on the tower. The wave gage buoy was deployed nearby. The resulting data supports a narrowing trend in the two-dimensional spectral width as a function of wavenumber. Two current spectral models support this to some extent, while other models do not. The data also shows a similar azimuthal width for the scatterometer return and the width of the short wavelength portion of the wave height spectrum after it has been averaged and extrapolated out to the appropriate Bragg wavelength. This appears to support current e.m. composite surface (two-scale) theories which suggest that the scattered return from the ocean at intermediate incidence angles is dominated by Bragg scattering which depends principally on the magnitude and shape of the two-dimensional wave height spectrum. However, the mean wind direction (which corresponds well with the peak of the scatterometer energy distribution) and the peak of 20 minute averages of the azimuthal energy distribution were out of alignment in two out of three data sets, once was by nearly $90^{\circ}$. There are a number of tenable explanations for this including instrument physical limitations and the possibility of significant surface currents, but none that would explain such a significant variation. Given that there are so few measurements of short wave directional spectra, however, these results should be considered preliminary in the field and more extensive measurements are required to fully understand the angular distribution of short wave energy and the parameters upon which it depends.
\end{abstract}

Thesis Supervisor: Dr. Jerome Milgram

Professor, Department of Ocean Engineering, MIT

Thesis Supervisor: Dr. Erik J. Bock

Interdisciplinary Center for Scientific Computing, University of Heidelberg 


\section{Acknowledgments}

Scientific and engineering accomplishments do not occur in a vacuum. I have been very fortunate to have been aided in my endeavors by a host of outstanding scientists, engineers, and family members.

Professor Jerome Milgram and Dr. Erik Bock, my thesis co-supervisors, have argued against, defended, struggled with, supported, and most importantly laughed at (and with) me at all the times when each was necessary. They both have my deepest respect and gratitude for their ability to let me find my own way (even when it wasn't the shortest path from $A$ to $B)$.

Other key contributors to my success include my office mate Sean Mckenna whose quiet patience and dilligence I hope to some day equal, my mentor Ken Prada whose integrity and friendship helped me remember why I began this journey in the first place, my soonto-be husband Al Tervalon who picked me up, dusted me off, and sent me back into battle the many times when I thought to surrender, and to my old (and new) family who have encouraged me to reach farther, see higher, and smile bigger.

Specific groups in the MIT and WHOI community have made my work not only successful, but enjoyable. They are the administrative staff in the Ocean Engineering department at MIT, the Carpenter's Shop, the Mechanical Shop, the Buoy Group, the Security Staff, Marine Operations, and the Education Office at WHOI.

Finally, I need to thank the many organizations who have provided funding for me to build my instrument and perform my experiments: the MIT Ocean Engineering Department, the WHOI Rienhart Coastal Research Center, the WHOI Education Office, the National Defense Science and Engineering Graduate Fellowship Program, and grant N0001493-1 0726 from the Office of Naval Research. 


\section{Contents}

1 Introduction $\quad 12$

2 Background and Theory $r$

2.1 Surface Wave Theory . . . . . . . . . . . . . . . . . 15

2.1.1 Wave Propagation . . . . . . . . . . . . . 15

2.1 .2 Energy Distribution . . . . . . . . . . . . . . . 16

2.2 Electromagnetic Scattering Theory and Remote Sensing . . . . . . . . . 22

2.2.1 General Theoretical Development . . . . . . . . . . . . . . 22

2.2.2 Mathematical Development . . . . . . . . . . . . . . . . . 24

2.3 Wave Measuring Instruments . . . . . . . . . . . . . . . 27

3 Circular Wire Wave Gage Buoy 29

3.1 Overview $/$ Introduction . . . . . . . . . . . . . . . . 30

3.1 .1 System Layout . . . . . . . . . . . . . . . . . . 30

3.1.2 Fundamental Operating Principle . . . . . . . . . . . . 30

3.2 Buoy Structure and Housings . . . . . . . . . . . . . . . . . 32

3.3 Analog Electronics (Filtering Package) . . . . . . . . . . . . . . 36

3.3 .1 Layout . . . . . . . . . . . . . . . . . 36

3.3.2 Filter and Modifications . . . . . . . . . . . . . . . 36

3.3.3 Noise Reduction and Signal Conditioning . . . . . . . . . . . . 38

3.4 Data Acquisition Hardware . . . . . . . . . . . . . . . . . 42

3.5 Power ................................. 43

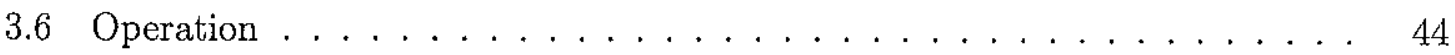


3.6.1 Startup and Data Download ... . . . . . . . . . . 44

3.6.2 Basic Commands, Calibration, and File Formats . . . . . . . . . 47

3.6 .3 Diagnostic Programs . . . . . . . . . . . . . . . . 51

3.7 Data Processing . . . . . . . . . . . . . . . . . . 52

3.7 .1 Process Description . . . . . . . . . . . . . . . 52

3.7 .2 Program Implementation $\ldots \ldots \ldots \ldots 59$

3.7.3 Calibration (step 2): postcal.for ........... 60

3.7.4 Calculation of Covariance: correl.c . . . . . . . . . . . 60

3.7.5 Calculation of Directional Spectrum: dfsum*.for ........ 61

4 Experimental Results $\quad 62$

4.1 Buzzards Bay Tower Experiment . . . . . . . . . . . . . . . . . . . 62

4.1 .1 Experimental Setup ........................ 62

4.1 .2 Buzzards Bay Tower NOAA Data . . . . . . . . . . . . . . 69

4.1 .3 Results . . . . . . . . . . . . . . . . . 70

4.2 Comparison of Data Processing Methods . . . . . . . . . . . . . . . 87

4.3 Discussion . . . . . . . . . . . . . . . . . 100

5 The Wave Height Spectrum and Scatterometry 102

5.1 The Wave Height Spectrum . . . . . . . . . . . . . . . . 103

5.1 .1 Frequency Spectrum . . . . . . . . . . . . . . . . . 103

5.1 .2 One- and Two-Dimensional Wavenumber Spectra . . . . . . . . . 106

5.2 Electromagnetic Scattering Models . . . . . . . . . . . . . . . . 122

5.3 Conclusions . . . . . . . . . . . . . . . . . 128

6 Summary 131

6.1 Future Work . . . . . . . . . . . . . . . . . . . 132

$\begin{array}{ll}\text { A Spectral Model References } & 142\end{array}$

$\begin{array}{ll}\text { B Scattering Model References } & 145\end{array}$

$\begin{array}{ll}\text { C Wave Measuring Instrument References } & 148\end{array}$ 
D Analog Electronics Interface Board Electrical Diagram 151

$\begin{array}{ll}\text { E Oscillator Circuit Electrical Diagram } & 153\end{array}$

F Data Acquisition Design Criteria $\quad 155$

G Data Acquisition Hardware Schematic $\quad 156$

$\begin{array}{ll}\text { H Data Acquisition Software } & 158\end{array}$

H.1 Wave.c . . . . . . . . . . . . . . . . . . . 158

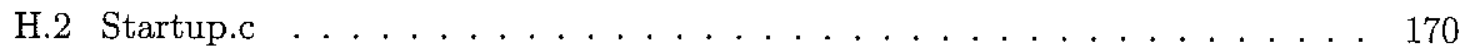

I Capacitive Wire Calibration $\quad 172$ 


\section{List of Figures}

2-1 Energy Density in Sea Surface Waves as a Function of Frequency [13] . . 15

3-1 Components of the Circular Wire Wave Gage Buoy . . . . . . . . . . . 30

3-2 Voltage Divider Relationship for Wire Wave Gage . . . . . . . . . . . 31

3-3 Top view and partial side view of the assembled circular wire wave gage buoy 32

3-4 Central Analog Electronics Housing . . . . . . . . . . . . . . . . 33

3-5 PVC Canisters for Batteries . . . . . . . . . . . . . . . . . . 34

3-6 Canister Mounting Brackets . . . . . . . . . . . . . . . . . . . 34

3-7 Data Acquisition Canister Endcap . . . . . . . . . . . . . . . 35

3-8 Image of Analog Electronics Boards Stacked for Installation in Buoy . . . . 37

3-9 Single Channel Front End Analog Electronics . . . . . . . . . . . . . . . . 39

3-10 Frequency Response for Analog Filtering of Each Channel . . . . . . . . . 41

3-11 Battery Wiring and Charging Diagram . . . . . . . . . . . . . . 44

3-12 Data Format for *. wav File . . . . . . . . . . . . . . . . . 50

3-13 Output File Format of wav2asci.exe Program . . . . . . . . . . . . 52

4-1 Buzzard's Bay Tower . . . . . . . . . . . . . . . . . . . 63

$4-2 \quad$ BUZM3 Instrument Layout, Top View . . . . . . . . . . . . . . . . . . 64

4-3 BUZM3 Instrument Layout, Side View . . . . . . . . . . . . . . . . . 64

4-4 Circular Wire Wave Gage Buoy Drift Track . . . . . . . . . . . . . . 66

4-5 Capacitive Wire Wave Gage Hexagonal Frame and Wire Location Diagram 68

4-6 Hourly Barometric Pressure and Air Temperature from BUZM3 Tower . . . 71

4-7 10 Minute Averaged Wind Direction and Velocity from BUZM3 Tower . . . 72 
4-8 CSAT3 Sonic Anemometer Data from Data Set 10281315 . . . . . . . . . 73

4-9 CSAT3 Sonic Anemometer Data from Data Set 10281344 . . . . . . . . . . 74

4-10 CSAT3 Sonic Anemometer Data from Data Set 10281414 . . . . . . . . . . 75

4-11 Wind Direction Histogram for Data Set 10281315 . . . . . . . . . . . 76

4-12 Capacitive Wire Wave Gage Single Wire Frequency Spectrum from Data Set

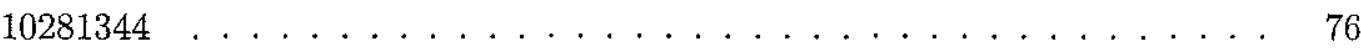

4-13 Capacitive Wire Wave Gage One-Dimensional Frequency Spectra Averaged From All 6 Wires From Data Sets 10281315, 10281344, and 10281414 . . . . 77

4-14 Capacitive Wire Wave Gage Two-Dimensional Frequency Spectrum from Data Set $10281315 \ldots \ldots \ldots \ldots \ldots$. . . . . . . . . . . . . . . . . . . . .

4-15 Capacitive Wire Wave Gage Two-Dimensional Frequency Spectrum from Data Set $10281344 \ldots \ldots \ldots \ldots$. . . . . . . . . . . . 80

4-16 Capacitive Wire Wave Gage Two-Dimensional Frequency Spectrum from Data Set $10281414 \ldots \ldots \ldots$. . . . . . . . . . . . . . . 81

4-17 Directional X-band Scatterometer Return from Data Set 10281315 . . . . . 83

4-18 Directional X-band Scatterometer Return from Data Set 10281344 . . . . . 84

4-19 Directional X-band Scatterometer Return from Data Set 10281414 . . . . . 85

4-20 Circular Wire Wave Gage Frequency Spectrum Averaged from 16 Single Wire Spectra from Data Sets 10281415, 10281445, and $10281515 \ldots \ldots$. . . . 86

4-21 Circular Wire Wave Gage Two-Dimensional Wave Height Spectrum from Data Set 10281415 (corresponding to 1315 EDT on October 28, 1998) . . 88

4-22 Circular Wire Wave Gage Two-Dimensional Wave Height Spectrum from Data Set 10281445 (corresponding to 1345 EDT on October 28, 1998) . . . 89

4-23 Circular Wire Wave Gage Two-Dimensional Wave Height Spectrum from Data Set 10281515 (corresponding to 1415 EDT on October 28, 1998) . . 90

4-24 Omnidirectional Spectra from the Circular Wire Wave Gage Data Sets 10281415, 10281445 , and $10281515 \ldots \ldots \ldots \ldots 1 \ldots \ldots \ldots \ldots$

4-25 Directional Slices of Circular Wire Wave Gage Two-Dimensional Wavenumber Spectra from Data Sets 10281415, 10281445, and 10281515 . . . . . . 92 
4-26 Scanning Laser Slope Gage Normalized Two-Dimensional Slope Spectrum Processed with SLSGproc from Data Sets ds023255.198 and ds023909.198 .

4-27 Scanning Laser Slope Gage Two-Dimensional Slope Spectrum Processed with CWWGproc from Data Sets ds023255.198 and ds023909.198 . . . . . . . . 97

4-28 Directional Comparison Between SLSGproc and CWWGproc . . . . . . . 99

4-29 Comparison of the Frequency Spectrum as Measured by the Capacitive Wire Wave Gage and the Circular Wire Wave Gage . . . . . . . . . . . . . . 101

5-1 Comparison of the DHH Frequency Spectrum Model with a Frequency Spectrum Measured at the Buzzards Bay Tower . . . . . . . . . . . . . 105

5-2 Comparison of the Omnidirectional Spectrum Models and Wavenumber Spectrum Slice Models with Data Collected at the Buzzards Bay Tower (10281415) 112

5-3 Comparison of the Omnidirectional Spectrum Models and Wavenumber Spectrum Slice Models with Data Collected at the Buzzards Bay Tower (10281445) 113

5-4 Comparison of the Omnidirectional Spectrum Models and Wavenumber Spectrum Slice Models with Data Collected at the Buzzards Bay Tower (10281515) 114

5-5 Comparison of the Normalized Directional Spreading Models with Data Collected at the Buzzards Bay Tower (10281415) . . . . . . . . . . . . 116

5-6 Comparison of the Normalized Directional Spreading Models with Data Collected at the Buzzards Bay Tower (10281445) . . . . . . . . . . . 117

5-7 Comparison of the Normalized Directional Spreading Models with Data Collected at the Buzzards Bay Tower (10281515) . . . . . . . . . . . 118

$5-8$ Buoy Orientation . . . . . . . . . . . . . . . . 120

5 9 Comparison of the Widths of the Directional Spreading Models with Data Collected at the Buzzards Bay Tower (10281415) . . . . . . . . . . . . 121

5-10 Convergence of Circular Wire Wave Gage Spectral Width Measurements . . 123

5-11 Directional X-Band Scatterometer Return from Data Set 10281315 . . . . . 124

5-12 Comparison of the Widths Between Spreading Models, the Circular Wire Wave Gage, and the X-Band Scatterometer (10281415 and 10281315)) . . 126

5-13 Comparison of the Widths Between Spreading Models, the Circular Wire Wave Gage, and the X-Band Scatterometer (10281445 and 10281344)) . . . 127 
D-1 Analog Electronics Interface Board . . . . . . . . . . . . . . . 152

E-1 Oscillator Circuit Diagram . . . . . . . . . . . . . . . 154

G-1 Data Acquisition Hardware Schematic . . . . . . . . . . . . . 157 


\section{List of Tables}

3.1 Circuit Diagrams and System Functions for Analog Filter . . . . . . . . 40

3.2 Data Acquisition Basic Specifications . . . . . . . . . . . . . . 43

3.3 Setup Configuration and User Prompted Information . . . . . . . . . . . . 46

3.4 Serial Console Configuration . . . . . . . . . . . . . . . . . 46

3.5 Command Line Options for wave. c . . . . . . . . . . . . . . . . 48

3.6 KVH C100 Compass Data Format . . . . . . . . . . . . . . . 51

4.1 Sonic Anemometer Calibration Coefficients . . . . . . . . . . . . 69

5.1 Directional Spreading Functions from Various Models . . . . . . . . . . 108

5.2 High Frequency Wavenumber Spectral Slice in the Peak Energy Direction from Various Models . . . . . . . . . . . . . . . . . . . . . . . . . . 109

A.1 Chronological Listing of Spectral Model References (1950's - 1990's) . . . . 142

B.1 Chronological Listing of Scattering Model References (1950's - 1990's) . . . 145

C.1 Chronological Listing of Wave Measuring Instrument References (1950's-1990's) 148

I.1 Calibration Data for Capacative Wire Wave Gage . . . . . . . . . . . . 172 


\section{Chapter 1}

\section{Introduction}

The emergence of airborne and space borne remote sensing capabilities have provided a longawaited tool for studying the surface and sub-surface properties of the world's oceans. This is because electromagnetic backscatter from the sea surface depends chiefly on the ocean surface roughness at both large and very small scales. The surface roughness is affected predominantly by the wind field, but also by other factors including surface currents, ocean floor topography, and surface films. Electromagnetic scattering techniques operated on satellite or airborne platforms have the capability, therefore, of providing information about these ocean processes on potentially synoptic scales. Understanding the mechanisms by which electromagnetic measurements are coupled to the surface roughness via the primary mechanism of Bragg scattering, and through the surface roughness to the actual wind field, surface currents, etc., however, is a very difficult task which requires detailed information about the surface wave field. While the large gravity waves which contribute to surface roughness have been under investigation for many years, technological advances allowed the study of the smaller capillary and capillary-gravity waves to begin in earnest only in the last three or four decades. As the theories describing electromagnetic wave interactions with the ocean surface have improved, however, so has the need for more and more detailed spatiotemporal information about the surface wave field.

The directional spreading of the surface spectrum has historically been the weakest aspect of spectral and scattering models because of the difficulty in obtaining at-sea measurements of it at small enough wavelengths. Toward that end, a new instrument has been 
developed to aid in the study of the directional characteristics of these very sort waves on the ocean surface. It is known as the circular wire wave gage. It is an autonomous buoy capable of providing directional energy distribution information about waves with wavelengths between 2 and 30 centimeters. This is the wavelength range which impacts the Bragg scattering mechanisms important in microwave backscatter. It was deployed on October 28, 1998 at the mouth of the Buzzards Bay in Massachusetts as part of an experiment designed to compare in situ measurements of both the surface wave height spectrum and electromagnetic backscatter with currently proposed models for both of these processes.

In this work the current theoretical knowledge about the surface wave height spectrum and electromagnetic backscatter from the ocean surface is coupled with in situ experimental data, providing evidence not previously available about the applicability and limitations of these theories. Chapter 2 presents the current surface wave and electromagnetic theories as well as describing other available techniques and instruments which have been used to measure short surface waves. A detailed description of the new instrument being used to measure directional distribution of short waves is given in Chapter 3. Chapter 4 describes the instruments deployed and the data collected during the Buzzards Bay Tower experiment. And finally, Chapter 5 ties the theory to the experimental data to illustrate the strengths and weaknesses of both. 


\section{Chapter 2}

\section{Background and Theory}

Accurate analytical and predictive models for ocean surface waves have been under development for many years. Long waves have historically received most of the attention because of the large amount of energy they contain, as is illustrated in Figure 2-1. The shorter waves saw little attention until World War II when the Navy became interested in understanding 'sea clutter', a radar phenomenon which could mask radar signatures of surface ships and submarines caused by wind roughened water surface $[14,69]$. Since that time much has been learned about short waves and their influence on important fields of study such as air-sea interactions (e.g. gas transfer) and wave growth and generation.

Many in situ and remote instruments have been developed to aid the study of these short waves. In order to understand the strengths and limitations of these instruments it is necessary to appreciate the physical phenomena they are measuring. The first section of this chapter, therefore, describes surface wave propagation and energy distribution. The remote sensing techniques used to study ocean surface waves require knowledge of the interaction of electromagnetic waves with the water surface. Accordingly, the second section describes electromagnetic scattering and radar cross section calculations. Finally, to add perspective to the comparison of instruments described later in this thesis, a section is included describing the historical development of wave measuring instruments and their capabilities. 


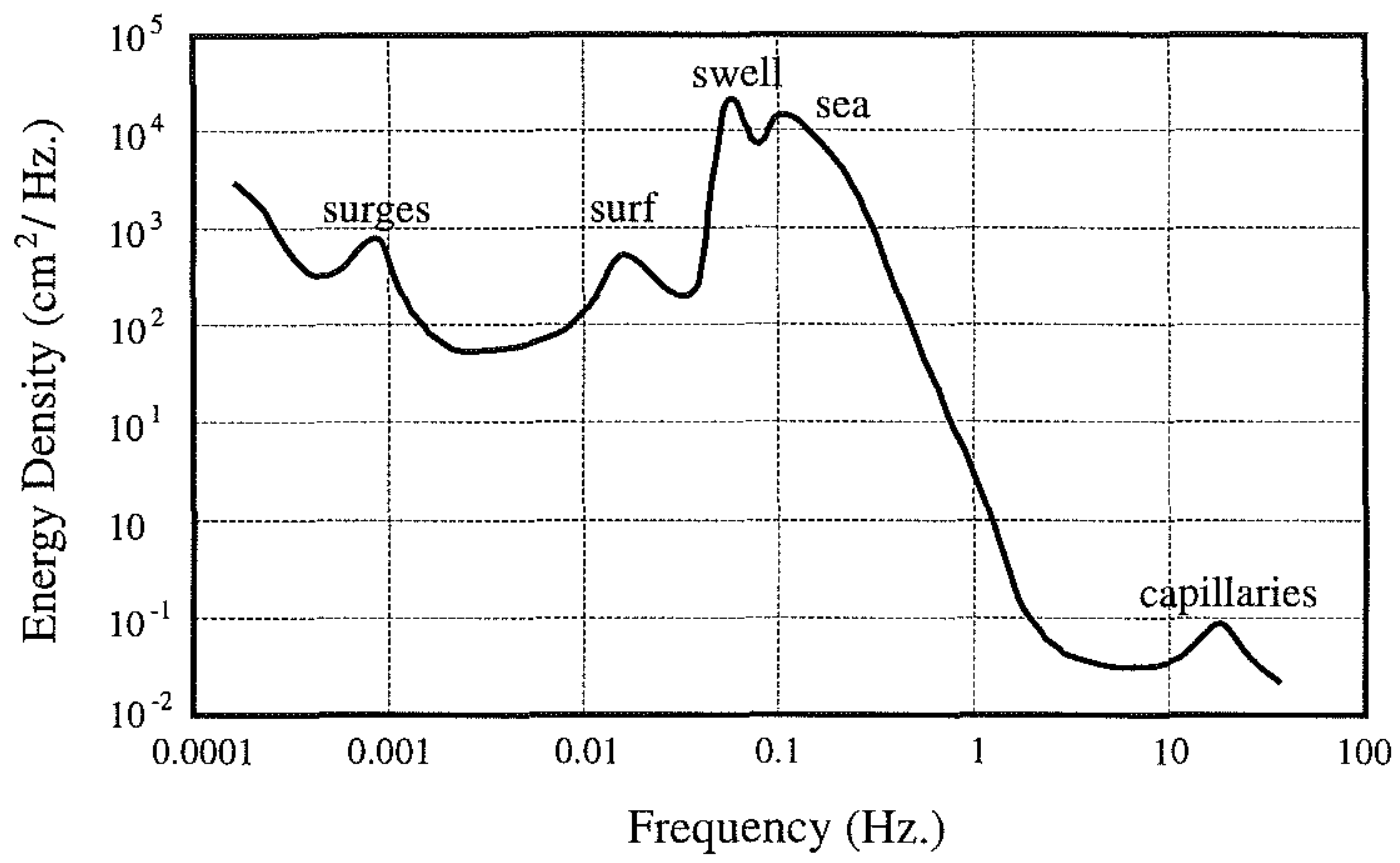

Figure 2-1: Energy Density in Sea Surface Waves as a Function of Frequency [13]

\subsection{Surface Wave Theory}

\subsubsection{Wave Propagation}

Ocean surface waves behave in a predictable manner (if one overlooks their generation and dissipation). While the ocean surface wave height as a function of space and time is a stochastic process [43] (see discussion below), the kinematics of freely-propagating waves is governed by the dispersion relation, which relates the frequency of a surface wave, $\omega$ $(\mathrm{rad} / \mathrm{s})$, to its wavenumber, $k(\mathrm{rad} / \mathrm{cm})$ where $k=2 \pi / \lambda$ with $\lambda=$ wavelength $(\mathrm{cm})$. For a linear, inviscid, irrotational flow, in the absence of currents, with both gravity and surface tension restoring forces, it takes the following form

$$
\omega^{2}=\left[g k+\frac{\tau k^{3}}{\rho}\right] \tanh (k h)
$$


In deep water $(k h \gg 1)$ this simplifies to

$$
\omega^{2}=g k+\frac{\tau k^{3}}{\rho}
$$

where $g$ is the gravitational constant $\left(981 \mathrm{~cm} / \mathrm{sec}^{2}\right), h$ is the water depth $(\mathrm{cm}), \tau$ is the surface tension $(\sim 73$ dynes $/ \mathrm{cm})$, and $\rho$ is the density of sea water $\left(\sim 1 \mathrm{~g} / \mathrm{cm}^{3}\right)$.

When $k \ll 3.7 \mathrm{rad} / \mathrm{cm}$ the gravitational term in equation 2.2 dominates and the waves are termed 'gravity waves'. For $k \gg 3.7 \mathrm{rad} / \mathrm{cm}$ the surface tension term dominates and the waves are known as 'capillary waves'. Because the demarkation between these regions is not very abrupt, waves with $k \sim 3.7$ are usually termed 'gravity-capillary' waves.

Equation 2.2 relates the wavelength of a particular wave to its frequency and, thus, relates the temporal characteristics of waves to their spatial characteristics. This is important in practice because measuring the temporal properties of surface waves is easier than measuring their spatial properties. It is, however, the spatial attributes of the gravity-capillary waves which dominate microwave electromagnetic scattering, as will be discussed in Section 2.2. Additionally, the dispersion relation is modified in the ocean environment because of the effects of surface currents, wave-wave interactions, and other nonlinear phenomena such as wave breaking. Even the linear processes, like Doppler shifting, alter the apparent frequencies (and wavelengths) of short waves making the transformation from the frequency (temporal) domain via the wavenumber (spatial) domain using the dispersion relation is of limited value. The significance of these difficulties will be explored further in Section 2.3 and is one of the motivations for the work presented in this thesis.

\subsubsection{Energy Distribution}

Assuming one can model an ocean surface wave field as a random process, the power (or wave) spectrum of the waves is described by the spatial and temporal energy distribution. As described by Kinsman [37] the covariance of the surface vertical displacement is defined as

$$
\rho(\boldsymbol{x}, \boldsymbol{r} ; t, \tau)=\overline{\eta(\boldsymbol{x}, t) \eta(\boldsymbol{x}+\boldsymbol{r}, t+\tau)}
$$


where $\eta$ is the sea surface vertical displacement as a function of position, $x$, and time, $t, r$ is the separation vector between two position vectors, and $\tau$ is the time separation. Its Fourier transform, known as the general three-dimensional power spectrum of the sea surface displacement, is

$$
\Phi(\boldsymbol{k}, \omega ; \boldsymbol{x}, t)=(2 \pi)^{-3} \iiint_{-\infty}^{\infty} \rho(\boldsymbol{x}, \boldsymbol{r} ; t, \tau) \exp [-i(\boldsymbol{k} \cdot \boldsymbol{r}-\omega \tau)] d \boldsymbol{r} d \tau
$$

where $k=\left(k_{x}, k_{y}\right)$ is the wavenumber vector, and $\omega$ is the frequency.

Many interesting aspects of the full three-dimensional spectrum can be visualized by reducing the dimensionality of the spectrum. These include the time evolution of wavenumber spectra for wave growth studies, the evaluation of wavenumber-frequency spectra for studies of free and bound waves, and the angular distribution of surface waves to study directionality and spreading. The reason for reducing the three-dimensional spectrum into a two-dimensional one in the context of the present study is that electromagnetic scattering theory, discussed in the next section, depends only on an instantaneous image of the sea surface, so the temporal variable is not needed for the scattering analysis. To this end, when one takes the three-dimensional spectrum from equation 2.4 and assumes a homogeneous and stationary wave field,

$$
\Phi(k, \omega ; \boldsymbol{x}, t) \Rightarrow \Phi(k, \omega)
$$

and then integrates out the frequency, $\omega$, one is left with

$$
\Phi(k)=(2 \pi)^{-2} \int_{-\infty}^{\infty} \rho(r) \exp [-i(k \cdot r)] d r
$$

where the surface covariance becomes

$$
\rho(\boldsymbol{r})=\overline{\eta(\boldsymbol{x}) \eta(\boldsymbol{x}+\boldsymbol{r})}
$$

which holds true if it is assumed that the sea surface is homogenous, implying that the surface covariance depends only on the relative lag between measurements $(\boldsymbol{r})$ and not the absolute location of the measurement $(x)$, and if it is assumed that the sea surface is 
stationary, implying that the surface covariance does not depend on time. Both of these assumptions are generally justified for sea states which are not fetch limited or which have a steady wind field. It is with these assumptions that surface wave height array data collected with a buoy deployed in relatively unchanging meteorological conditions over a limited period of time can be considered statistically homogeneous or consistent.

The two-dimensional spectrum from equation 2.6 describes how each wavenumber component of the surface wave field contributes to the total mean square wave energy, $\overline{\eta^{2}}$ where

$$
\overline{\eta^{2}}=\iint_{k} \Phi(k) d k
$$

Numerous experiments have been conducted to measure this two-dimensional wavenumber spectrum. Many have measured time series wave heights at an array of locations, either in wave tanks or at-sea, and used the dispersion relation to transform the results into the wavenumber domain. For the long wave portion of the spectrum this has been very successful. Other experiments have used remote sensing instruments such as scatterometers or radars to try to solve the inverse problem: given a measured scattered return and a model for the surface scattering as a function of the two-dimensional wavenumber spectrum, among other things, what is the appropriate wavenumber spectrum which would reproduce the observed scattered return? Each experiment has helped generate a better defined model of the 'true' two-dimensional ocean wave height spectrum.

In this model generation process a common means of modeling the wavenumber spectrum has developed. It uses a slice of the wavenumber spectrum in the direction of the dominant wind waves at the spectral peak in conjunction with a spreading function which models the directional spreading of the energy on the surface. The result is

$$
\Phi(k)=\Phi\left(k_{x}, k_{y}\right) \Rightarrow \Phi(k, \theta)=\Phi\left(k, \theta_{\max }\right) D(k, \theta)
$$

where $\theta_{\max }$ is the direction around which the majority of the energy in the spectrum is centered, and is believed to be closely aligned with the wind direction. The shape of the wavenumber spectrum for the short wave region in the dominant wave direction has 
generally taken the form

$$
\Phi\left(k, \theta_{\max }\right) \propto k^{-m}
$$

where $m$ has taken on values from 3 to 5 , depending on the experimental conditions [48, $46,7,2]$. The proportionality constant often includes parameters such as wind speed, wind stress, fetch, peak spectral frequency, and/or wave age.

The early models for the directional spreading were based on low frequency limits and low angular resolution using pitch-and-roll buoys such as the work of Longuet-Higgins et al. [40] who modeled the directional spread as a function of frequency

$$
D(f, \theta)=A \cos ^{2 s}\left(\frac{\theta-\theta_{\max }}{2}\right)
$$

where $s$ was determined through a parameterized fit to the data. Later models modified the $s$-parameter, such as that of Hasselmann et al. [26], but agreement on a consistent behavior for $s$ has not been achieved. Because of this inability to adequately define $s$, Donelan et al. [17] (DHH) proposed an alternate form of the spreading function. Their new model was based on the idea that envelope soliton groups of Stokes waves propagating around the main wave direction can be described by a hyperbolic secant. The energy distribution about the primary long wave direction should, therefore, also take the form of a hyperbolic secant, assuming that the wave field consists primarily of these envelope solitons and noting that they survive interactions with each other. $\mathrm{DHH}$ showed that their proposed form of the spectrum

$$
D(\omega, \theta)=A \cosh ^{-2}(\beta \theta)
$$

where $\beta$ is a function of the frequency ratio $\omega / \omega_{\text {peak }}$ and takes on different values in different $\omega / \omega_{\text {peak }}$ regions, fit their capacitive wire wave gage array data more accurately than the previous $\cos ^{2 s}(\theta / 2)$ form did.

As more in situ experimental data was collected at higher frequencies and with better angular resolution, however, it became apparent that the $\mathrm{DHH} \beta$ was not universally ap- 
plicable. Furthermore, Banner [3] disagreed with the DHH extrapolation of $\beta$ to higher wavenumbers using a cutoff criterion which limited spreading at higher wavenumbers and found that when he used the DHH spreading function without the cutoff, he was able to fit both the old and newly available data more accurately with

$$
\beta= \begin{cases}2.28\left(k / k_{p}\right)^{-0.65} & \text { for } 0.97<k / k_{p}<2.56 \\ 10^{-0.4+0.8393 \exp \left[-0.567 \ln \left(k / k_{p}\right)\right]} & \text { for } k / k_{p}>2.56\end{cases}
$$

where $k_{p}$ is the wavenumber at the peak of the spectrum.

Apel [1] followed in Banner's footsteps but chose to use a Gaussian form of the spreading function which matches the Banner modification to the DHH spreading function to within experimental errors. He additionally extended its applicable range out to the gravitycapillary region based on tank tests conducted by Jähne and Riemer [34]. He also used his resulting twomdimensional spectrum as an input to a scattering model and compared the results with available radar data. (Previous models had been very loose when it came to representing the two-dimensional spectrum either as a function of $\omega$ or $k$, but as data has become available in the higher wavenumber region, all the new models use only the wavenumber form for the reasons described earlier.) The spreading function Apel used is

$$
D(k, \theta)=\exp \left(\frac{-\left(\theta-\theta_{\max }\right)^{2}}{2 \theta_{s}^{2}(k)}\right)
$$

where

$$
\frac{1}{2 \theta_{s}^{2}(k)}=\left[0.14+5.0\left(k / k_{p}\right)^{-1.3}\right]
$$

Elfouhaily et al. [19], after analyzing the Bjerkaas and Riedel spectrum [7], the Donelan and Pierson spectrum [18], and the Apel spectrum [1], proposed yet another form of the spreading function based primarily on the Apel shape. This new function, however, included an important aspect that the previous models did not: it required that the spreading function be centrosymmetric (symmetric through the origin). This property is imperative if the spectral model is to be used in electromagnetic scattering models (a key aspect of this thesis) because the electromagnetic scattering theory depends heavily on the Fourier 
transform of the height covariance function. Because the directional spectrum is the Fourier transform of the two-dimensional height covariance function (as was defined previously), it is, by definition, a real and even function, leading to the symmetry requirements on the spreading function. Elfouhaily et al., thus, restructured the form of the spreading function so that

$$
D(k, \theta)=\frac{1}{2 \pi}[1+\Delta(k) \cos (2 \theta)]
$$

When the spreading functions of Mitsuyasu et al., Hasselmann et al., Donelan et al., and Apel are modified to fit Elfouhaily's form they all involve the hyperbolic tangent. Recognizing their contributions, but also their limitations, Elfouhaily et al. modeled $\Delta$ as

$$
\Delta(k)=\tanh \left[a_{0}+a_{p}\left(c / c_{p}\right)^{2.5}+a_{m}\left(c_{m} / c\right)^{2.5}\right]
$$

where $a_{0}=\ln (2) / 4, a_{p}=4, a_{m}=0.13 u^{*} / c_{m}$, and $c_{m}=0.23 \mathrm{~m} / \mathrm{s}$.

Except for Apel's model, the previously described directional spreading models were based on parameterized fits to in situ experimental data. An alternate method for measuring the directional spectrum on the ocean surface is to use remote sensing tools and an inverse processing technique. This will be described in more detail in Section 2.2. Two recent authors have used this method and added their own modifications to Apel's spectrum. Caudal and Hauser [9] argue that the spreading proposed by Banner cannot be extrapolated past the short gravity wavelengths. They choose, therefore, to add a modifying term, $\delta$, to Apel's spectrum which functions to narrow the spectrum at higher wavenumbers.

$$
\frac{1}{2 \theta_{s}^{2}(k)}=\left[0.14+5.0\left(k / k_{p}\right)^{-1.3}+\delta\right]
$$

where his resulting form of $\delta$ after incorporating the directional spectrum into a scattering model and comparing it to $\mathrm{P}$ and $\mathrm{K}_{a}$-band high-frequency radar data is

$$
\delta=10^{\left(p_{1} X^{2}+p_{2} X+p_{3}\right)}
$$

where $X=\log _{10}(k), p_{1}=-0.177, p_{2}=1.11$, and $p_{3}=-2.00$. 
Finally, Romeiser et al. [55], accepted the basis of the Apel form of Donelan's spectrum, but noted that it did not accurately reproduce measured cross-wind/downwind ratios measured with scatterometers from $\mathrm{L}$ to $\mathrm{K}_{a}$-band. The model they propose takes the following form

$$
\begin{aligned}
\frac{1}{2 \theta_{s}^{2}(k)}= & 0.14+0.5\left[1-\exp \left(-\frac{k u_{10}}{c_{1}}\right)\right] \\
& +5 \exp \left[2.5-2.6 \ln \left(\frac{u_{10}}{u_{n}}\right)-1.3 \ln \left(\frac{k}{k_{n}}\right)\right]
\end{aligned}
$$

where $c_{1}=400 \mathrm{rad} / \mathrm{s}, k_{n}=1 \mathrm{rad} / \mathrm{m}, u_{n}=1 \mathrm{~m} / \mathrm{s}$.

A more comprehensive reference list of the development of spectral models over the last five decades is included in Appendix A.

\subsection{Electromagnetic Scattering Theory and Remote Sensing}

Remote sensing instruments, such as radars and scatterometers, generate electromagnetic radiation, direct it at the ocean surface, and then use the backscattered or reflected signals to discern information about ocean surface properties. Interpreting the reflected signals from the surface requires detailed analytical analysis and a thorough understanding of electromagnetic surface scattering theory. The theory of electromagnetic scattering from the ocean surface will be discussed first, and then its mathematical development and application using the normalized radar backscatter cross section will be explained.

\subsubsection{General Theoretical Development}

The first scattering theories were based on geometrical optics arguments which assumed that the reflective surface was smooth, in the sense that the radius of curvature of the surface was large in comparison to the impinging electromagnetic wavelengths. These theories were only able to accurately predict scattering at near normal incidence angles, however, because they modeled the sea surface as being composed only of very long waves. It was later found that short surface waves with wavelengths near the wavelength of the incident electromagnetic field contributed significantly to the backscattered return at moderate in- 
cidence angles. To try and incorporate these small waves a new theory was presented in the 1950's which described the scattering surface as being composed of small perturbations from a smooth, possibly undulating surface [54]. This model, describing 'slightly rough surfaces', used the concept of Bragg type scattering which results from coherent or resonant reflection off of an ordered surface. Bragg scattering predicts that the scattered return from a roughened sea will be proportional to the (two-dimensional) Fourier transform of the surface elevation spatial covariance function at a particular wavenumber. The wavenumber is dependent on the electromagnetic wavelength used by the scatterometer or radar, and the angle of incidence of the radiated beam. Furthermore, if the sea surface is in motion, the scattered return will be Doppler shifted by a frequency associated with its wavelength and the velocity of the waves upon which the Bragg scatterers are traveling. The limitations of this method are the product of its assumptions of small surface displacements compared to the electromagnetic wavelength (inherent in all perturbation approaches) and small surface slopes. While a reasonable approximation, these assumptions do not always apply to realistic sea surfaces, particularly as they become rough due to increased wind speeds. More refined theories were therefore formulated to remedy the discrepancies between observation and mathematical description.

Research in the 1960's showed that these limitations could be overcome if the 'slightly rough surfaces' theory was further modified by assuming that the large scale waves on the sea surface could be broken into small patches of tangent planes upon which the shorter scale scatterers propagated $[4,5,69]$. The perturbation theory, therefore, is applied to individual small patches that are summed to get the total scattered return, assuming that the short waves decorrelate very quickly relative to the large scale waves. This theory, known as composite surface theory or two-scale theory, has been used as a basis for most of the recent models of sea surface scattering $[67,18,52,39]$ despite the limitations imposed by its linear assumption which comes primarily from the limitation that the small patches must be uncorrelated. At the very large and very small incidence angles, higher order approximations using surface currents [56], modulation effects [25, 58], bound or parasitic capillary waves [53,47], and specific wave shape features such as wave crests [36] and bores $[64,42]$ are in development but have not been applied generally in scattering analysis. 


\subsubsection{Mathematical Development}

Maxwell's equations form the basis of electromagnetic theory. A number of texts describe how they can be used with the appropriate boundary conditions and assumptions to develop simple scattered field equations from a smooth surface for both vertically and horizontally polarized incident plane waves $[51,65]$. These scattered field equations can then be used to determine the mean power received by an antenna from a surface. This is accomplished by integrating the scattered field equations over the antenna aperture weighted by the antenna beam pattern, and then normalizing appropriately. Using the slightly rough surface theory assumptions (and assuming the sea surface is homogeneous) it can then be shown that the non-dimensional normalized radar cross section of a slightly rough saltwater surface at moderate incidence angles can be expressed as [50]

$$
\sigma_{0}(\phi, \theta)=8 \pi k_{0}^{4} G(\phi) \Gamma(\phi, \theta)
$$

where $k_{0}$ is the electromagnetic wavenumber in units of radians/length and $G(\phi)$ is a nondimensional polarization dependent geometric reflection coefficient incorporating the dielectric constant such that

$$
G_{H}(\phi)=\frac{\cos ^{4}(\phi)}{[1+(1 / \sqrt{\epsilon}) \cos (\phi)]^{4}}
$$

and

$$
G_{V}(\phi)=\frac{\cos ^{4}(\phi)\left(1+\sin ^{2}(\phi)\right)^{2}}{[\cos (\phi)+(1 / \sqrt{\epsilon})]^{4}}
$$

where $\phi$ is the incidence angle in radians, $\theta$ is the azimuthal look direction in radians, $\epsilon$ is the non-dimensional relative dielectric constant of sea water [57], and finally

$$
\Gamma(\phi, \theta)=\Phi\left(2 k_{0} \sin (\phi), \theta\right)+\Phi\left(2 k_{0} \sin (\phi), \theta+\pi\right)
$$

which has units of length ${ }^{2} /(\text { radian/length })^{2}$. These equations show that the normalized radar cross section from an air-sea interface using the Bragg backscattering model is directly 
proportional to the wavenumber spectrum evaluated at the Bragg resonant wavenumber, $k_{B}=2 k_{0} \sin (\phi)$.

As was noted in Section 2.2.1, when the small slope assumptions are violated, as they are when the small scale roughness 'rides' on longer swell, the pure Bragg model can no longer be used. A modified Bragg model called the two-scale or composite surface theory is used instead. This model takes into account the long wave slope through a probability function as well as altering the apparent incidence and azimuthal look directions at which the terms are evaluated. Mathematically it can be derived from the simple Bragg model by taking a few simple steps. To begin with, the plane of incidence must be tilted to incorporate the long wave slope. Let the long wave slope in the plane of incidence be $\gamma_{L y}$ and out of the plane of incidence be $\gamma_{L x}$. The new local incidence angle then becomes

$$
\phi^{\prime}=\phi-\gamma_{L y}
$$

Furthermore, tilting out-of-plane is equivalent to rotating the tilted surface by a small angle $\theta^{\prime}$ where for small angles $\cos \theta^{\prime} \approx 1$ and $\sin \theta^{\prime} \approx \gamma_{L x} / \sin \phi^{\prime}$ [69]. Now, for a vertically polarized incidence field

$$
\vec{E}=E \sin \theta^{\prime} \hat{a}+E \cos \theta^{\prime} \hat{b}
$$

where $\hat{a}$ is a unit vector lying in the tilted surface and perpendicular to the incident electromagnetic field vector and $\hat{b}$ is a unit vector lying in the local plane of incidence and perpendicular to the incident electromagnetic field vector. The resulting incident vertically polarized electromagnetic field is, thus, a combination of both vertical and horizontal polarizations. The same can be shown for the horizontal incident field. Propagating these modifications through the slightly rough surface equations generates the normalized radar backscatter cross section for the composite surface theory

$$
\sigma_{0}(\phi, \theta)=8 \pi k_{0}^{4} \int G\left(\phi, \gamma_{L x}, \gamma_{L y}\right) \Gamma\left(\phi^{\prime}, \theta^{\prime}\right) P\left(\gamma_{L x}, \gamma_{L y}\right) d \gamma_{L x} d \gamma_{L y}
$$

where $P\left(\gamma_{L x}, \gamma_{L y}\right)$ is the probability distribution of the long wave slopes, and it is generally 
assumed that $\Gamma\left(\phi^{\prime}, \theta^{\prime}\right)$ can be approximated by $\Gamma\left(\phi^{\prime}, \theta\right)$. Additionally, the $G\left(\phi, \gamma_{L x}, \gamma_{L y}\right)$ function is defined as

$$
G_{H}\left(\phi, \gamma_{L x}, \gamma_{L y}\right)=\left\{\sqrt{G_{H}\left(\phi^{\prime}\right)}+\left[\frac{\gamma_{L x}}{\sin \left(\phi^{\prime}\right)}\right]^{2} \sqrt{G_{V}\left(\phi^{\prime}\right)}\right\}^{2}
$$

and

$$
G_{V}\left(\phi, \gamma_{L x}, \gamma_{L y}\right)=\left\{\sqrt{G_{V}\left(\phi^{\prime}\right)}+\left[\frac{\gamma_{L x}}{\sin \left(\phi^{\prime}\right)}\right]^{2} \sqrt{G_{H}\left(\phi^{\prime}\right)}\right\}^{2} \approx G_{V}\left(\phi^{\prime}\right)
$$

since for most incidence angles $G_{V} \gg G_{H}$. From these last two equations it can be seen that the tilt modulation induced by the long wave slopes affects the horizontally polarized return much more than the vertically polarized return. For further detail and explanation of this derivation see reference [51].

Plant [50] went a step further with this derivation by using the Cox and Munk [14] Gaussian approximation of the slope probability distribution and evaluated the integral in equation 2.28. The results were simplified by Lyzenga [41] assuming a long wave zero mean slope and presented in the following form

$$
\bar{\sigma}_{0}(\phi, \theta)=\sigma_{0}(\phi, \theta)\left[1+\frac{\mu_{1}}{2}\left\langle\gamma_{L y}^{2}\right\rangle+\frac{\mu_{2}}{2}\left\langle\gamma_{L x}^{2}\right\rangle\right]
$$

where

$$
\mu_{1}=\frac{1}{\sigma_{0}}\left(\frac{\partial^{2} \sigma_{0}}{\partial \phi^{2}}\right)=\frac{G^{\prime \prime}(\phi)}{G(\phi)}+2 \frac{G^{\prime}(\phi) \Gamma^{\prime}(\phi, \theta)}{G(\phi) \Gamma(\phi, \theta)}+\frac{\Gamma^{\prime \prime}(\phi, \theta)}{\Gamma(\phi, \theta)}
$$

and

$$
\mu_{2}=\frac{1}{\sigma_{0}}\left(\frac{\partial^{2} \sigma_{0}}{\partial \alpha^{2}}\right)=\frac{2(2 \sqrt{R}-1)}{\sin ^{2}(\phi)}
$$

where $\left\langle\gamma_{L y}^{2}\right\rangle$ is the in-plane long wave slope variance, $\left\langle\gamma_{L x}^{2}\right\rangle$ is the out-of-plane long wave slope variance, $R=G_{V} / G_{H}$ for horizontal polarization, $R=G_{H} / G_{V}$ for vertical polarization, and $\alpha=\tan ^{-1}\left(\gamma_{L x}\right)$ which is the tilt angle perpendicular to the plane of incidence [41]. 
Equation 2.31 is an approximation to second order in the tilting angles which can be used to estimate of the influence of long wave slope variations on the normalized radar backscatter cross section.

Other non-linear and non-Bragg mechanisms which have been postulated to affect the radar backscatter cross section will not be addressed in this thesis, but a detailed reference list of the development of scattering models over the last five decades is included in Appendix B.

\subsection{Wave Measuring Instruments}

Many instruments have been developed to measure sea surface spectral characteristics to test and provide data to verify scattering theories. The variety of methods used includes aerial photogrammetry based on sun glint [14], stereo photography based on surface slope [28], resistive wire wave staff measurements [38], capacitive wire wave staff measurements of surface height $[45,17]$, radar and scatterometry measurements $[59,68,21]$, single point laser slope measurements of surface height $[63,29]$, scanning laser slope measurements $[70,32,8]$, and surface imaging devices using video processing [33, 71]. A more detailed review of the development of wave measuring instruments over the past five decades is included in Appendix C.

Each of the techniques described above has its drawbacks. The wire wave gage tools suffer from problems such as disturbing the wave field itself, limited resolution of wave height or wave slope, and difficulties accommodating the many orders of magnitude that wave heights span (i.e. long waves with amplitudes of meters and capillary waves with amplitudes of millimeters). The laser devices can generally only measure slope (although some single point devices can be made with laser ranging capabilities), and many have limitations on the time of day they can be deployed due to the interference of sunlight with optical sensors. Additionally, the laser systems do not deal well with the change in focal length caused by water surface height variation over the sensor area. The remote techniques (photogrammetry, stereo photography, radar and scatterometer methods, surface imaging) suffer from difficulties in absolute calibration, and, in the case of scatterometry, lack of full 
understanding of electromagnetic scattering from the ocean surface. All extant sensors also have difficulties or outright failures when measuring cresting or breaking waves.

The resistive circular wire wave gage described in Chapter 3 is not exempt from the limitations listed above. It is, however, different from the instruments referenced in Appendix $\mathrm{C}$ in that it combines the simplicity of wire wave gages with the higher spatial and directional resolution of recent laser scanning slope gages. This directional resolution combined with the inadequate understanding and experimental data to describe the spreading function described in Section 2.2 makes the comparison of coincident data collected with both the circular wire wave gage and the X-band scatterometer instruments a unique opportunity to improve the current state of knowledge in this area. 


\section{Chapter 3}

\section{Circular Wire Wave Gage Buoy}

The circular wire wave gage buoy and its data processing algorithms were designed to generate an estimate of the two dimensional sea surface spectrum. Details of the prototype design and testing of the circular wire wave gage can be found in the author's Masters thesis [61]. The prototype gage was designed as a proof-of-concept instrument and successfully achieved this goal. It was not, however, constructed with the attributes necessary for its proposed use in the scientific study described in this document. The study involved experiments conducted at sea which have formed the fundamental contribution of this research to the fields of oceanographic remote sensing and surface wave studies. The primary deficiencies of the prototype instrument included the fragile nature of its structural design, the need for an external deployment platform, inconsistent electrical performance, the lack of an external data acquisition system, and finally, the absence of an internal power source.

This chapter details the development of the circular wire wave gage buoy beyond its prototype phase. It describes the system layout, the instrument's basic components, how it is operated, how the data is processed, as well as the major modifications made which facilitated its use in the experiments described in Chapter 4. 
Buoy Structure and Housings

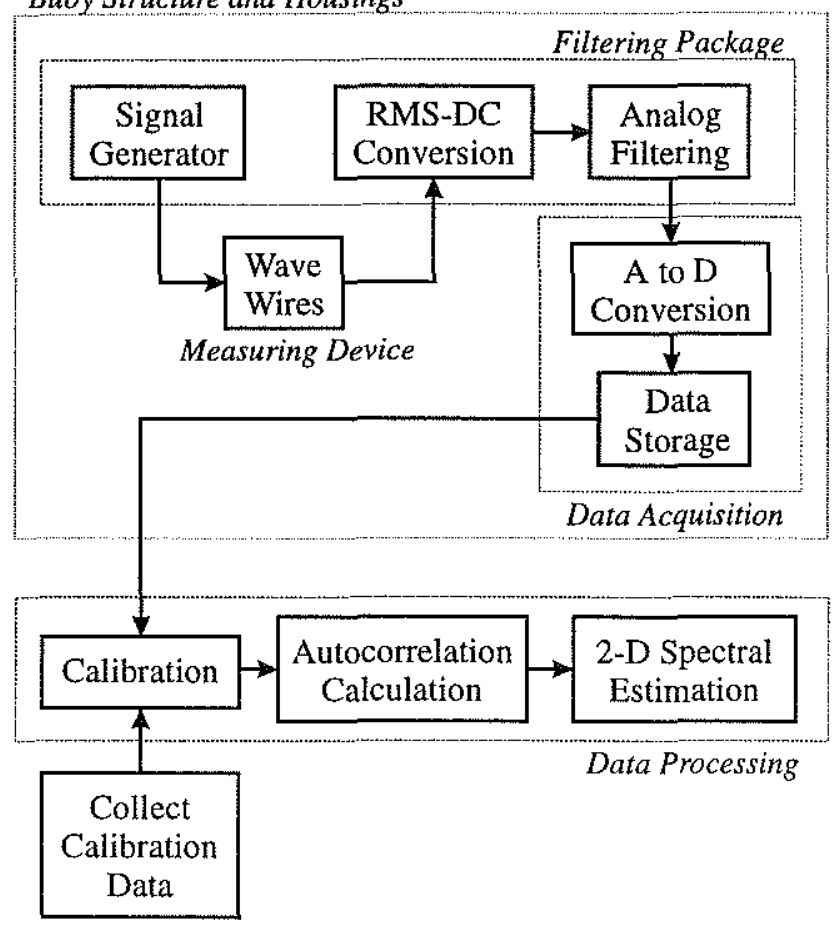

Figure 3-1: Components of the Circular Wire Wave Gage Buoy

\subsection{Overview/Introduction}

\subsubsection{System Layout}

The system, including both the instrument and data processing algorithms, is comprised of five major components: the filtering package, the measuring device, the data acquisition system, the buoy structure and housings, and the data processing package. The relationship between these components is schematically represented in Figure 3-1. Each of the components is described in detail in this chapter.

\subsubsection{Fundamental Operating Principle}

The key component of the wave wire buoy is its measuring device: the 64-element wave wire array. Each of the 34-gauge nickel chromium (nichrome) wave wires has a resistance of 17 ohms/foot and acts as a voltage divider as shown in Figure 3-2. The relationship between 


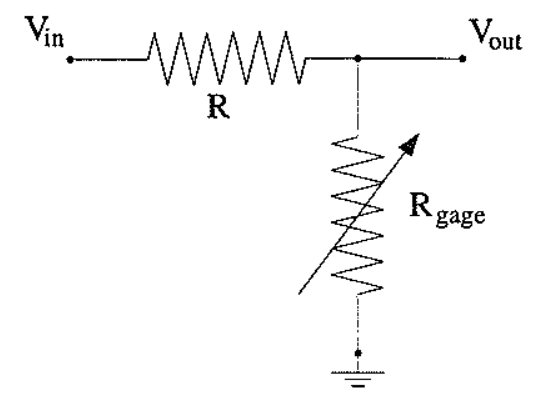

Figure 3-2: Voltage Divider Relationship for Wire Wave Gage

the input and output voltages of a voltage divider is mathematically represented by

$$
V_{\text {out }}=\frac{R_{\text {gage }}}{R+R_{\text {gage }}} V_{\text {in }}
$$

where $R$ is a fixed resistance internal to the wave gage circuitry and $R_{\text {gage }}$ is the resistance of the wave wire. If $R$ is significantly greater than $R_{\text {gage }}$, this relationship can be approximated by

$$
V_{\text {out }} \approx \frac{R_{\text {gage }}}{R} V_{\text {in }}
$$

Because $R$ is fixed, the output voltage will be nearly linearly related to the input voltage through $R_{\text {gage }}$. The seawater, due to its high conductivity, effectively acts as a short circuit between the point where each resistive wire pierces the water surface, and the aluminum center post of the gage which is connected to the electrical ground of the electronics package. As the level of the seawater on each of the wires changes, the effective resistance of each of the wires, $R_{\text {gage }}$, changes, varying the voltage measured across the voltage divider. The voltage measured across each wire is, thus, linearly related to the sea-surface elevation at that point. This linear relationship allows the filtered output signal from the wave gage to be used to reconstruct the input surface elevations. These surface elevations are then used to generate an estimate of the directional sea surface spectrum which is the ultimate product of the instrument and its processing algorithms. 


\subsection{Buoy Structure and Housings}

The circular wire wave gage mechanical design was based around the original circular wire layout arranged by former MIT graduate student, David Chen which arranged the 64 wire wave gages in a 20 centimeter diameter circle [12]. This layout facilitates the data processing and is fairly straightforward to implement. The remainder of the buoy design was chosen primarily to support and locate the wave wires, and additionally to minimize the flow disturbance around them while maintaining a stable long wave following platform. A number of additional constraints on the design were also incorporated. These included low cost, due to limited funding, and ease of deployment, assembly, and disassembly, because of intended single or two-person use and testing. The resulting buoy design is shown in Figure 3-3.

The water-tight central housing contains the pre-processing analog electronics, compass, and temperature probe. The structure also functions as a support and locator for the 64 nichrome wires. It is constructed entirely of aluminum and is shown in Figure 34.

The three canisters which form the base corners of the pyramid of the buoy contain

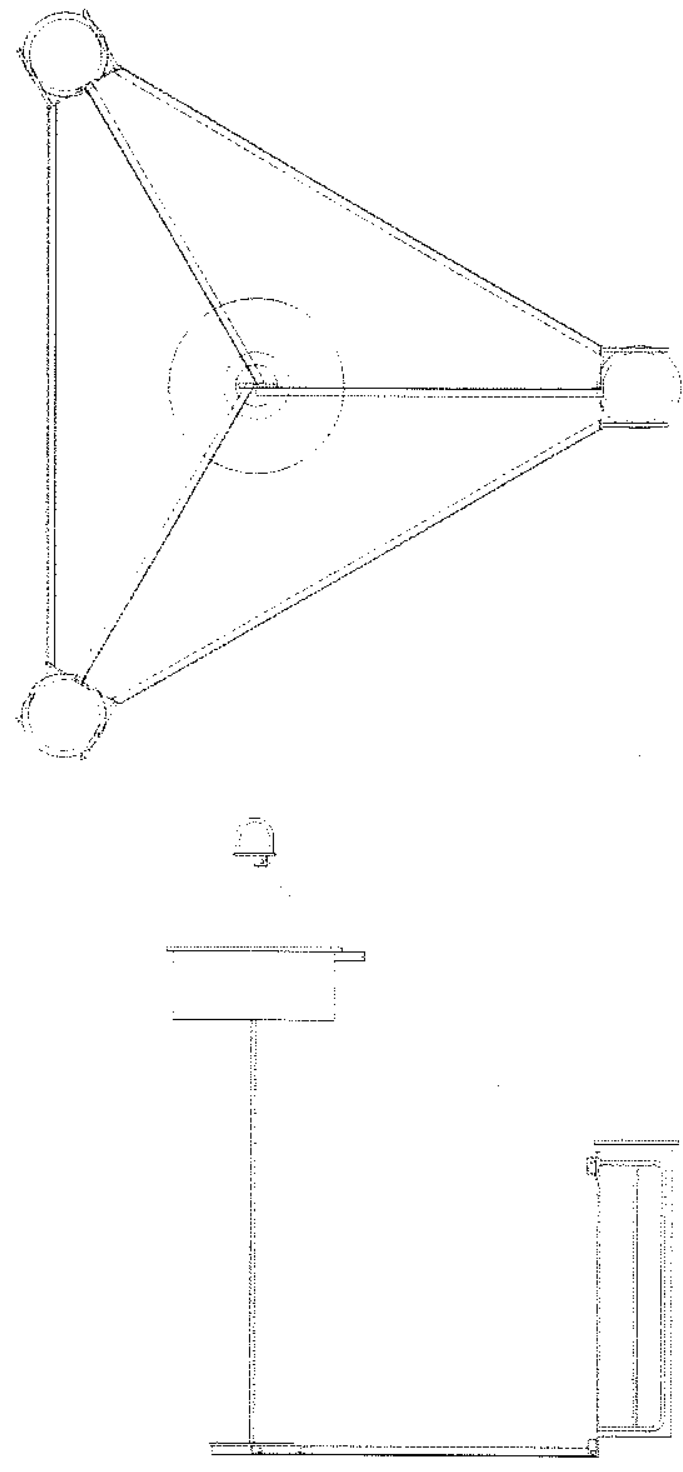

Figure 3-3: Top view and partial side view of the assembled circular wire wave gage buoy. Note that the wave wires (not shown) surround the vertical post of the central filter housing. 


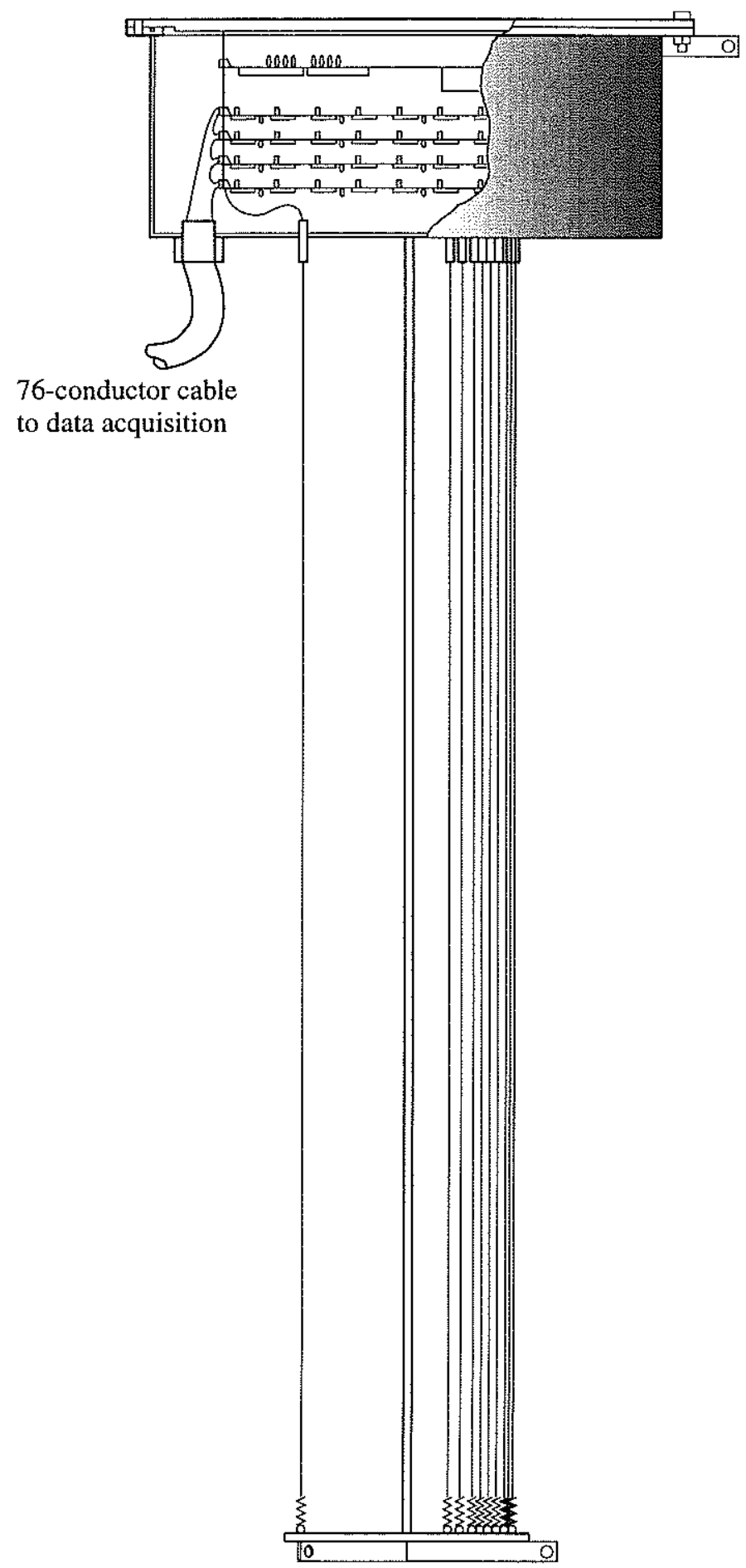

Figure 3-4: Central Analog Electronics Housing 
the data collection system and the batteries. Up to four 12-volt rechargeable leadacid batteries are secured in each of the two battery mounting brackets and loaded into two of the canisters which are constructed of PVC and are shown in Figure 3-5. This allows the flexibility of using up to eight batteries depending on the particular deployment specifications (e.g. duration of deployment, weight considerations, etc.). A vent port in the lid of each battery canister allows for any out-gassed fumes from the batteries to be discharged between buoy deployments and during battery recharging. The third canister houses the PC104 form factor data acquisition system and is constructed of aluminum. The size and weight of each of the three housing with its contents are nearly equal (when using four batteries in each of the battery canisters), reducing the need for additional ballasting. The mounting brackets used to secure the PVC and data acquisition canisters to the buoy are shown in Figure 3-6. Hose clamps are run through the vertical oval holes and around the canisters to keep them from moving after assembly. Figure 3-7 displays the data acquisition canister endcap and describes its ports.

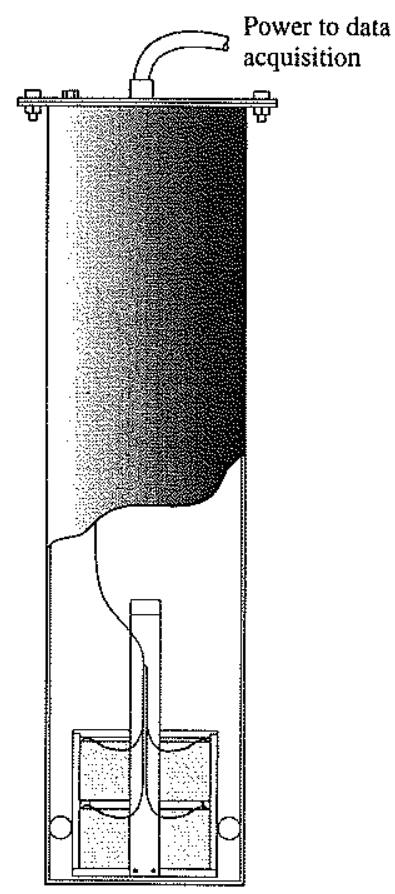

Figure 3-5: PVC Canisters for Batteries
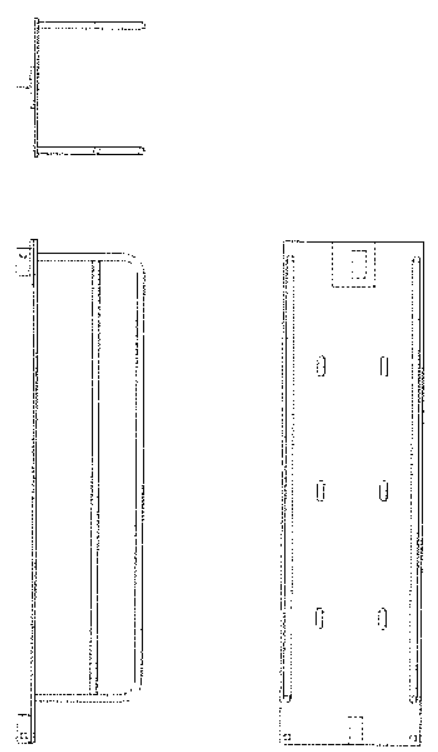

Figure 3 6: Canister Mounting Brackets 


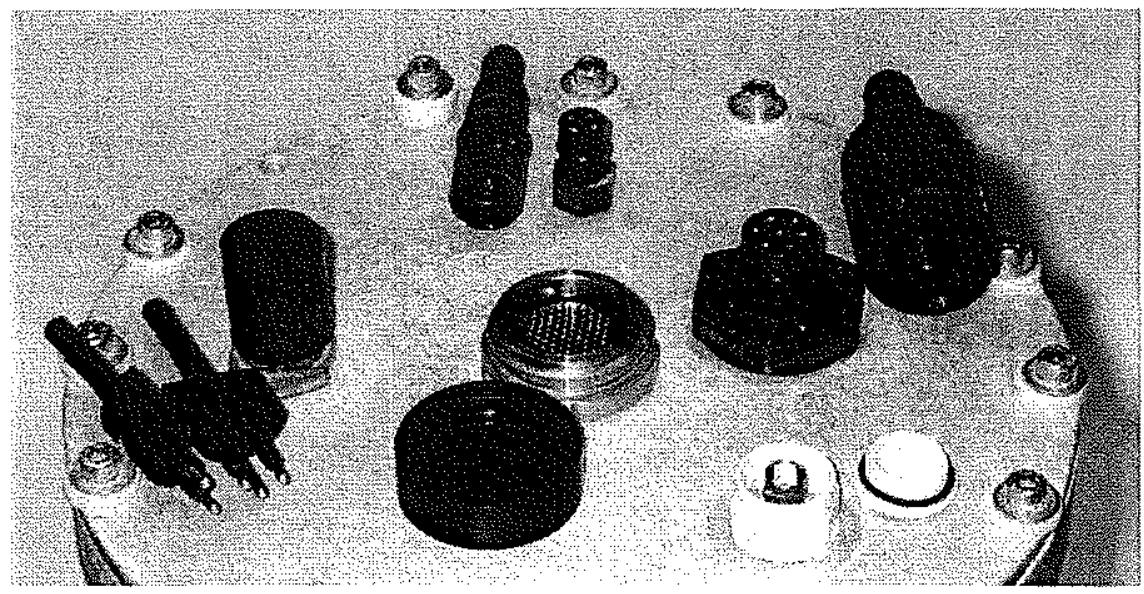

Figure 3-7: Data Acquisition Canister Endcap. Proceeding clockwise from the top center connector: Serial Port 4-pin connector, Unused 7-pin connector, Ethernet 10Base2 connector, Battery 4-pin connector (one 2-pin male connector comes from each battery housing), and in the center, Analog Electronics Output 76-pin connector. The corresponding dummy plugs and watertight covers are shown next to each connector.

The superstructure is constructed of aluminum and fiberglass angle. The angle is bolted to the canisters and the central housing during assembly. While a fixed/welded structure would have been more rigid, the bolted construction was chosen because it would have been awkward to transport and work on the assembled instrument due to its large size (2.1 meters on a side). The resulting modular nature of the super-structure allows easy assembly, disassembly, and transport.

When deployed, the buoy floats with about half the length of the nichrome wave wires exposed above the mean water level. It follows the long waves (waves with wavelengths greater than 5 or 6 meters) very smoothly, and has almost no vertical motion when only high frequency (very short) waves are present. In choppy seas with 2 to 5 meter intermediate steep waves, however, it has difficulty keeping the floatation canisters from dipping below the water surface because of its light weight and broad footprint. For these reasons it can be deployed both in the open ocean and in coastal waters, but should not be deployed in seas where short steep waves or whitecapping is occuring. 


\subsection{Analog Electronics (Filtering Package)}

Many modifications were made to the original pre-processing analog electronics of the instrument. A number of these were based on the prototype performance such as improvements in noise reduction, changes to the analog filter shape, and reduction of analog system power draw. They were originally secondary to the fundamental matter of simply building a buoy which functioned mechanically, and building the new stand-alone data acquisition system. Once the new system was assembled it worked well in the lab, but nearly half the channels were severely attenuated when it was deployed in the ocean. Because of the difficulty in establishing the cause of the attenuation, some of the originally proposed modifications to the analog electronics and numerous new changes were implemented to solve this problem.

\subsubsection{Layout}

The analog electronics are distributed on five PC boards which are stacked horizontally on top of one another. Figure 3-8 shows this configuration. Four of the boards are identical and each contains sixteen identical channels of analog filtering. The fifth board contains the power conditioning, the calibration switch, the flux gate compass, the temperature probe, and acts as the interface between the filtering boards and the data acquisition package, and between the filtering boards and the wire wave gages. The electrical diagram for this interface board is shown in Appendix D.

\subsubsection{Filter and Modifications}

The generation and filtering of each of the 64 channel voltage outputs is accomplished in several steps which are illustrated in the electrical circuit diagram shown in Figure 3-9. First, a $10 \mathrm{KHz}$ oscillating voltage is generated. This carrier signal is common to all the channels. The signal is then passed through a gain adjust, and sent to each of the nichrome wave wires. The water height on each wire amplitude modulates the high frequency carrier. The output of the wave wire voltage divider circuit discussed in Section 3.1.2 is then passed through a gain adjust and then through an RMS-to-DC converter which filters out the carrier frequency and leaves the envelope (the amplitude modulated signal). The signal is 


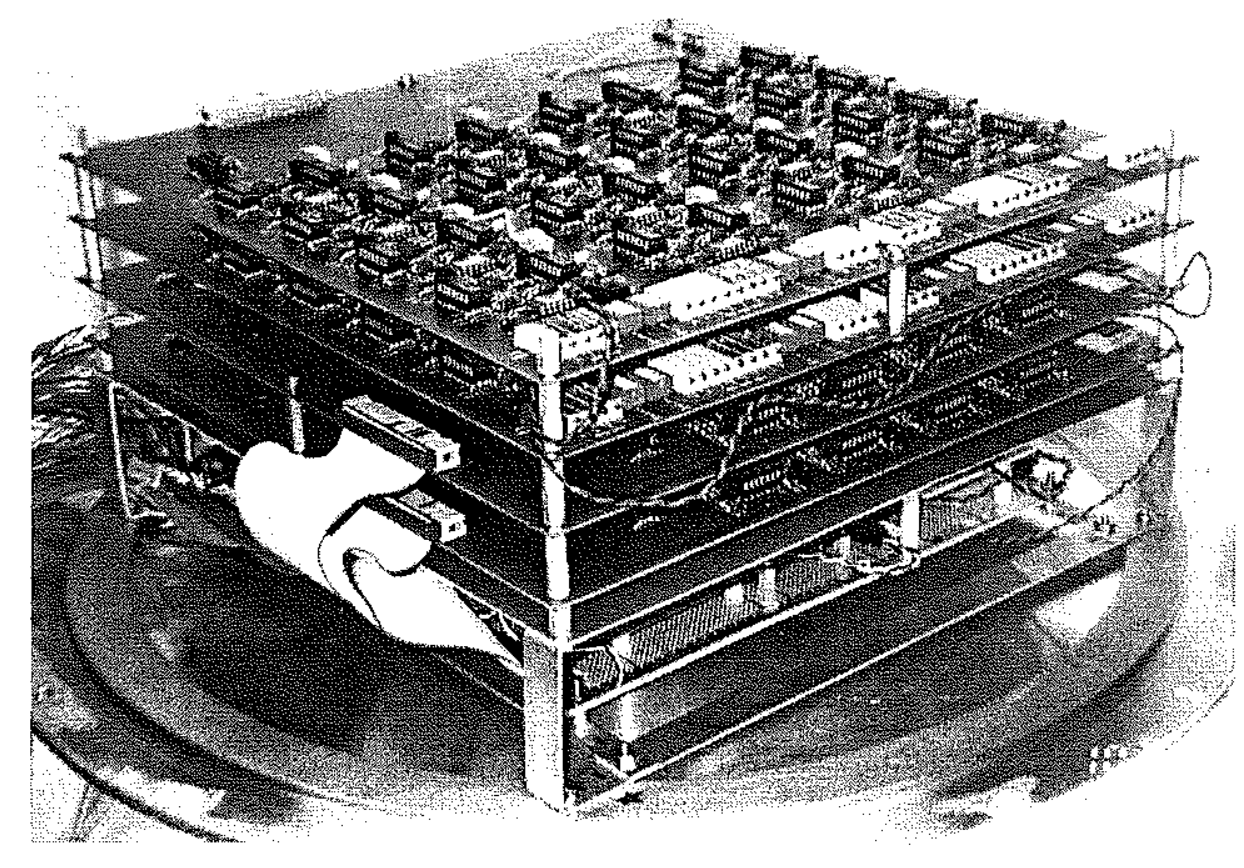

Figure 3-8: Image of Analog Electronics Boards Stacked for Installation in Buoy. The large distance between the lowest board and the four filtering boards is to accommodate the compass. The upper copper shield board is not shown. 
next sent through a trim offset and gain adjust, and then through a bandpass filter. The gain of the signal is again adjusted, and it is sent through a noise rejection low pass filter before finally being sent to the analog-to-digital converters in the data acquisition system.

Modifications made to the original analog electronics are readily apparent when comparing Figure 3-9 with Figure 3.5 from the author's Masters thesis [61]. The key differences are the addition of capacitors across the feedback loops of the filters, new operational amplifiers, and the modification of the filter cutoff frequency and shape accomplished by modifying the resistor and capacitor components of the filters. The manual calibration switch has also been changed to a relay controlled switch, allowing it to be computer controlled. Details about the calibration switch and oscillator input can be found in Appendices D and E.

The system function of the filter electronics, representing the ratio between the Laplace transform of the response of the system to the transform of the source [60], can be mathematically estimated using the nominal values of the circuit elements. Table 3.1 shows the circuit diagrams and resulting mathematical forms of the system functions for the individual steps in the filtering process. Combining all the individual filter system functions by multiplying them together gives the system function for the entire bandpass filter. Substituting in the appropriate element values and evaluating along the frequency axis $(s=j w)$ results in the sinusoidal steady state frequency response of the analog filter for each channel. A plot of this appears in Figure 3-10. The filter shape was chosen primarily to whiten the input energy in the low frequency range. This is necessary because as can be seen from Figure 2-1, the region of the spectrum from $0.3 \mathrm{~Hz}$ out to about $2 \mathrm{~Hz}$ includes nearly four orders of magnitude in energy. In order to resolve this vast range with a data acquisition system having limited resolution it was necessary to whiten the input. It is also important to note that given the Nyquist frequency for the data sampling of $50 \mathrm{~Hz}$, it is evident that the analog filter is adequately designed to prevent any significant aliasing from high frequency input or noise.

\subsubsection{Noise Reduction and Signal Conditioning}

While the noise in the prototype analog electronics was acceptable, since a new housing and power control board were being developed, some effort was spent trying to reduce the noise 


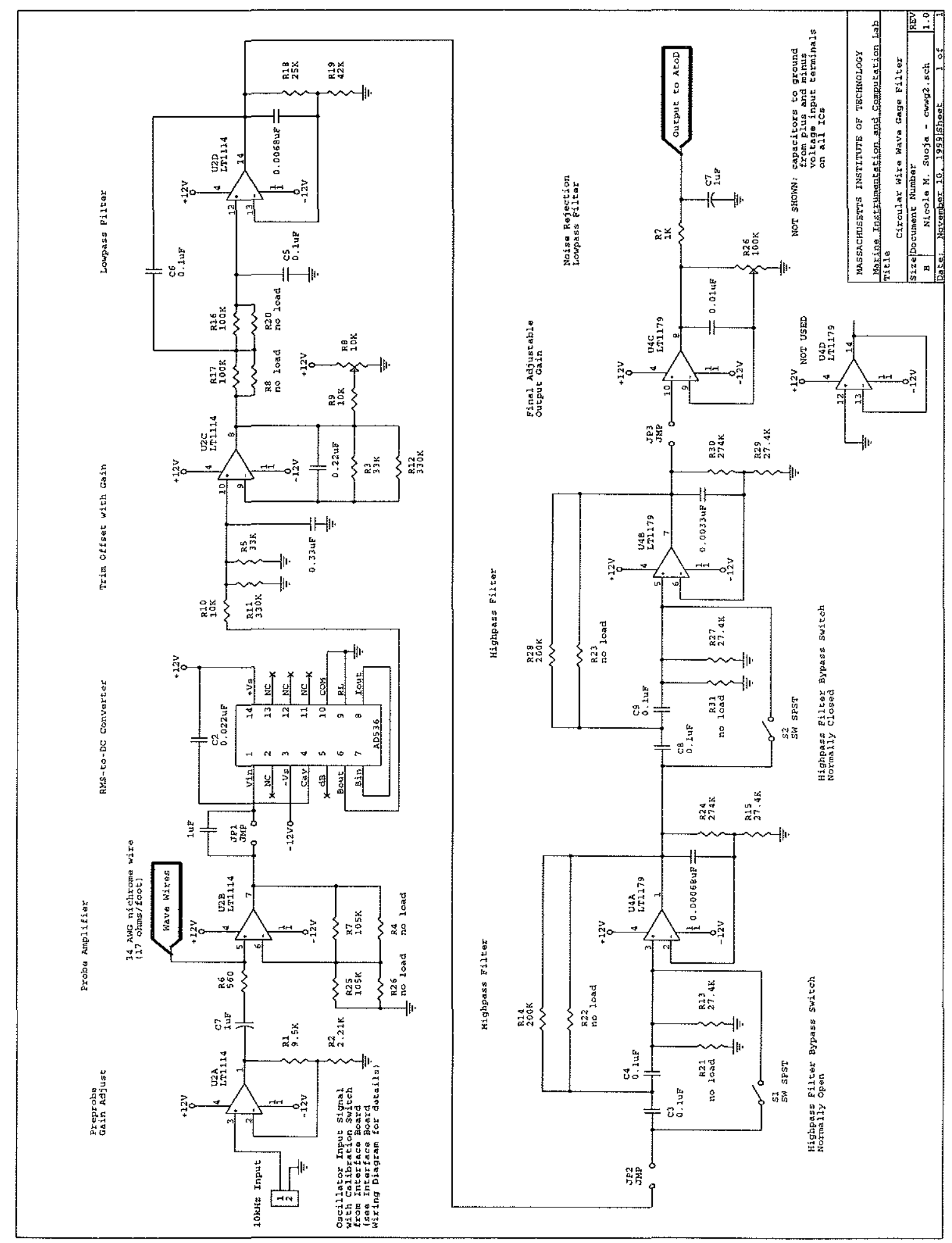

Figure 3-9: Single Channel Front End Analog Electronics 


\begin{tabular}{|c|c|c|}
\hline Name & Circuit Diagram & System Function \\
\hline $\begin{array}{l}\text { Low pass } \\
\text { Filter }\end{array}$ & Rid & 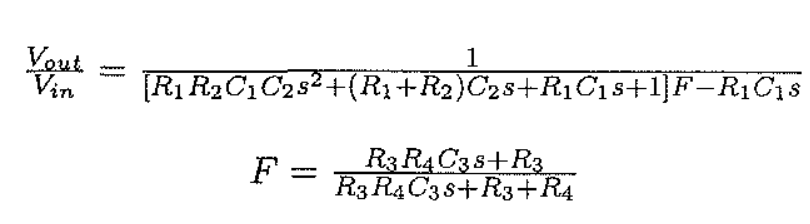 \\
\hline $\begin{array}{l}\text { First } \\
\text { High pass } \\
\text { Filter }\end{array}$ & & $\begin{array}{c}\frac{V_{\text {out }}}{V_{\text {in }}}=\frac{R_{1} R_{2} C_{1} C_{2} s^{2}}{\left[R_{1} R_{2} C_{1} C_{2} s^{2}+\left(R_{1} C_{1}+R_{1} C_{2}+R_{2} C_{2}\right) s+1\right] F-R_{2} C_{2} s} \\
F=\text { same as above }\end{array}$ \\
\hline $\begin{array}{l}\text { Second } \\
\text { High pass } \\
\text { Filter }\end{array}$ & $R_{2}$ & $\frac{V_{\text {out }}}{V_{\text {in }}}=\frac{R_{4}}{R_{4} R_{3} C_{3} s+R_{3}}+1$ \\
\hline $\begin{array}{l}\text { Final } \\
\text { Gain } \\
\text { Adjust }\end{array}$ & $\mathrm{R}_{2}$ & $\frac{V_{\text {out }}}{V_{\text {in }}}=\frac{R_{1}}{R_{1} R_{2} C s+R_{2}}+1$ \\
\hline $\begin{array}{l}\text { Noise } \\
\text { Rejection } \\
\text { Filter }\end{array}$ & 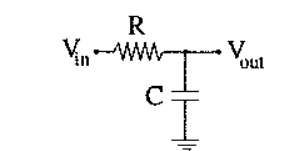 & $\frac{V_{\text {out }}}{V_{\text {in }}}=\frac{1}{R C s+1}$ \\
\hline
\end{tabular}

Table 3.1: Circuit Diagrams and System Functions for Analog Filter 

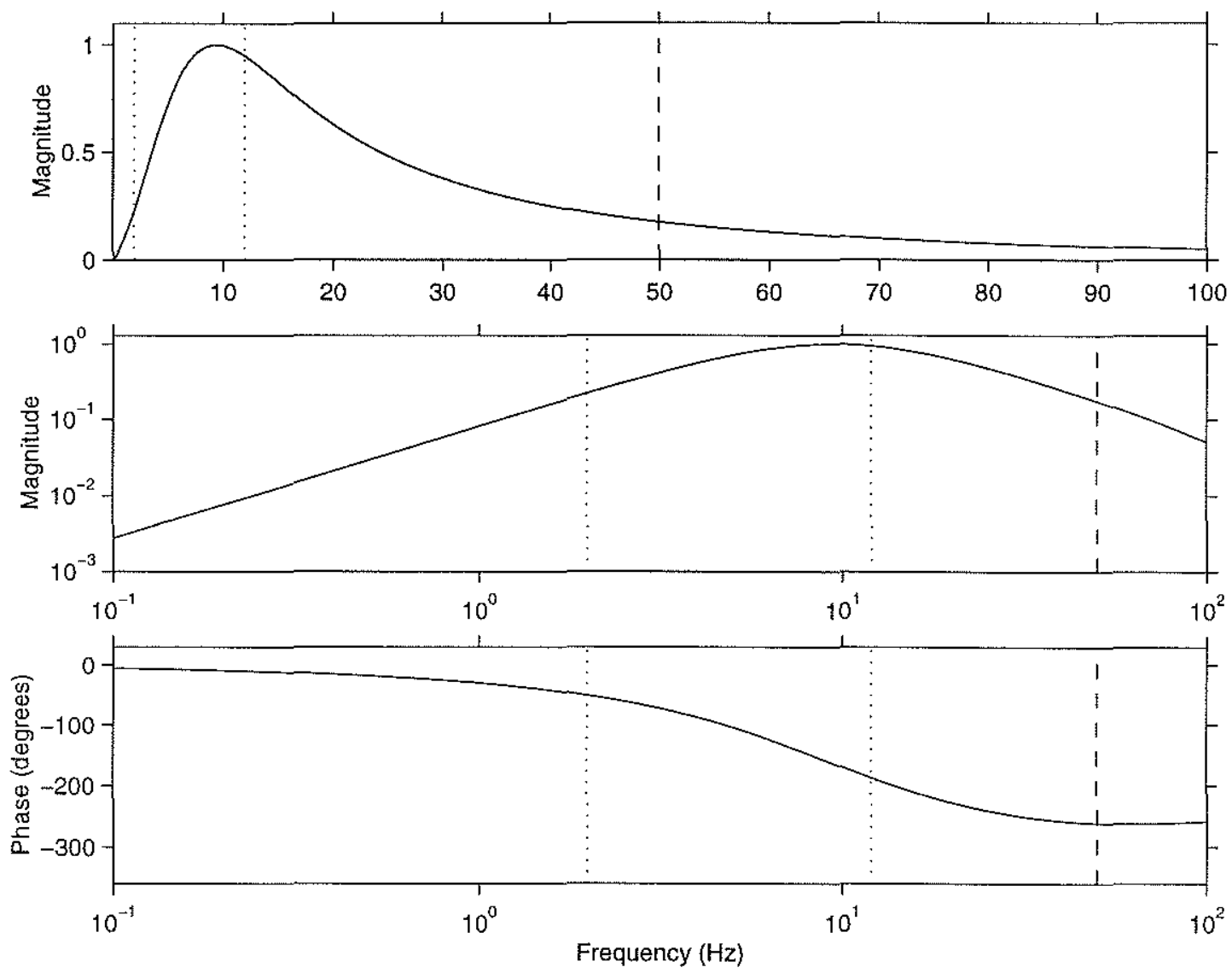

Figure 3-10: Frequency Response for Analog Filtering of Each Channel. The short dashed lines represent the approximate limits of the digital bandpass filter used in the post processing. The long dashed line represents the Nyquist frequency of the data acquisition system. The $\mathrm{x}$-axis is shown in log scale for the lower two plots. These functions are very reproducible from channel to channel. 
even further. To this end, the following modifications were made to the analog system. Some of these modifications were made to help solve the attenuation problem mentioned earlier.

1. Capacitors were added between the plus and minus voltage supplies and ground on all the integrated circuit (IC) chips.

2. New amplifier ICs were used which reduced the quiescent current draw by each amplifier by more than a factor of ten.

3. Plain PC boards with a thin sheet of copper which are the same physical dimensions as the electronic boards were mounted on either end of the electronics stack and grounded to the housing.

4. A ground wire was added to each of the four electronics boards connecting it directly to the housing.

5. The cable running from the analog electronics housing to the data acquisition system was fully shielded.

6. A temperature probe was placed in the analog electronics housing to monitor the environmental temperature of the capacitors so that any temperature changes which might affect the value of the capacitors (which are temperature sensitive) can be taken into account during calibration.

\subsection{Data Acquisition Hardware}

The data acquisition system was developed to sample and store a set (or scan) of the 64 analog outputs at $100 \mathrm{~Hz}$ (i.e. the beginning of each scan is triggered every 0.01 seconds, but the collection of the 64 analog voltages occurs much faster than that). It also collects data from the on-board compass, the batteries, and the temperature probe. It was designed, built, and programmed by Kenneth Prada, at the Woods Hole Oceanographic Institution. The specifications and performance of the system are detailed in Table 3.2. A complete list of the design criteria and constraints is presented in Appendix F, and a schematic of the data acquisition wiring diagram can be found in Appendix G. 


\begin{tabular}{|rl|}
\hline Stack configuration: & PC/104 \\
Processor: & CoreModule 486-II 50 MHz \\
A/D Boards (4 each): & 16 channel RTD DM5406 \\
Network board: & Ampro MiniModule/Ethernet \\
Hard drive: & Western Digital 540 MB, Model WDAL2540 \\
Batteries (8 each): & Yuasa NPH5-12, 12V 5.0Ah rechargeable \\
\hline \hline Data acquisition speed (per scan): & $100 \mathrm{~Hz}$ \\
Burst rate for sampling purposes: & $100 \mathrm{kHz}$ \\
\hline
\end{tabular}

Table 3.2: Data Acquisition Basic Specifications

\subsection{Power}

The wave gage is powered by up to eight 12 volt rechargeable batteries. These batteries are mounted in two wooden storage racks which fit snuggly into the PVC housings to prevent movement during deployment. The four batteries in each housing are wired in parallel with appropriate diodes to allow for recharging and prevent them from discharging each other. The wiring diagram for this arrangement is shown in Figure 3-11. The batteries are typically charged between each deployment. To do this, the vent port in each battery housing is opened and the positive terminal of the charging circuit, which is attached to the vent plug, and the ground connector on the battery output cable are connected to a battery charger. The maximum current which should be used is 0.5 amps per battery, and the maximum voltage charge should be set at 15.7 volts. This will charge the batteries to 13.8 volts, their maximum rated charge, and will allow for losses in the system due to the diodes and load resistors. After a full day's deployment the batteries may need to charge for up to ten hours.

The entire system (digital and analog) draws approximately 19 watts when running. With eight batteries fully charged and a safety factor of two, the instrument can collect over 12 hours of data. In order to conserve power during a deployment, a Watchdog timer is used which shuts down the power to all the systems between data sets, and then restarts them at a user specified interval, or until a user specified number of data files has been collected (see Section 3.6.1). This power save mode allows the instrument to be deployed for longer durations than it would be limited to if it were collecting data continuously. It was found, however, that in productive coastal waters the wires became coated with film 


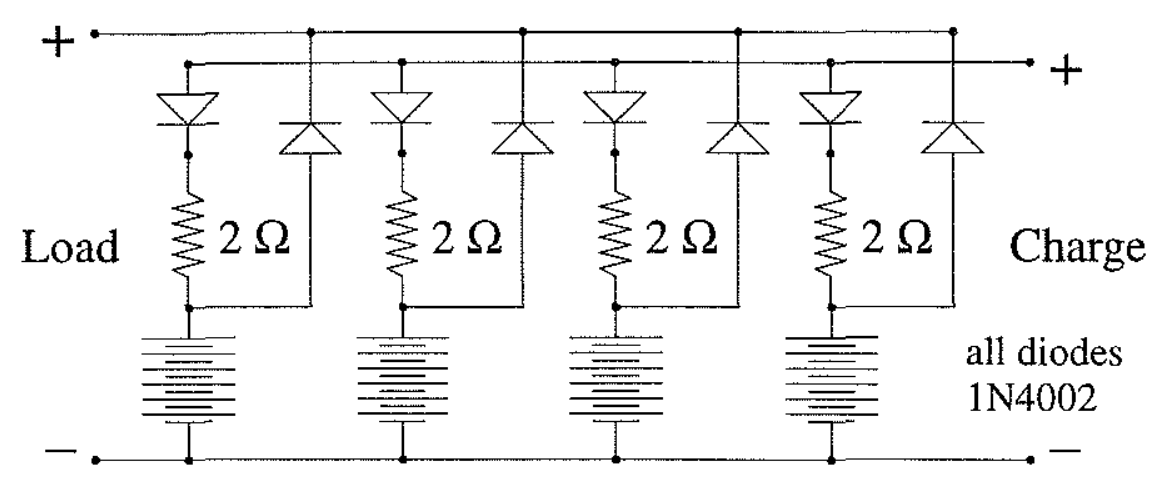

Figure 3-11: Battery Wiring and Charging Diagram

from the water surface, which affected their ability to accurately measure wave height after only about four to five hours, so the deployment duration is not generally controlled by power limitations.

\subsection{Operation}

The wave gage buoy is controlled by two programs. The primary program, wave.c, is the data acquisition code which collects a single data set including signal outputs from the 64 wave wires, the temperature probe, the compass, and the battery voltage. A batch file, run_wave.bat, contains the call to wave.c and its command line options. The secondary program, setup.c, sets the start time for the first call of run_wave, the interval between the beginning of each call to run wave, and the total number of data sets to be collected. Copies of wave.c and setup.c can be found in Appendix H. Once all the data files have been collected, the system shuts down and does not 'wake up' again. Once the data has been collected it can be downloaded through a local network which can be accessed via a 10Base2 port on the data acquisition housing.

\subsubsection{Startup and Data Download}

The data acquisition (daq) system is turned on by plugging the power cords from the two battery housings into their connector on the daq housing endcap. The computer system operates in two basic modes: data collection mode and serial console mode. In the data 
collection mode, which is the default, the system turns on, collects data based on the user specified startup configuration settings, and then goes to sleep (shuts down all power). In the serial console mode the user must be connected to the buoy through a serial cable and have a serial console program running on the externally connected computer. When the system turns on, the user is given three choices for operation, the 'operate' state (data collection mode), the 'normal' state (dummy terminal access to the wave gage buoy computer, i.e. $\mathrm{C}: \backslash$ prompt), or the 'PC-Anywhere' state, which runs the setup programs on the buoy to allow it to be accessed using $\mathrm{PC}$-Anywhere as an alternate means of transferring data to and from the buoy. Data can be downloaded via a 10Base-2 network connection when one is in the serial console mode running in the 'normal' state.

\section{Startup}

The user must do two things to set the buoy up to collect data autonomously. Both must be done in the serial console mode. First, the length of each of the data sets must entered into the run.wave bat program by setting the number of blocks to collect (see Section 3.6.2). This batch file contains only one line:

wave -b ???

where the questions marks are replaced with the appropriate number of blocks to collect.

The second thing the user must do is run the setup program. The setup configuration program is accessed by running the setup. exe program from the $\mathrm{C}: \backslash$ prompt. A description of the program output and expected user input is detailed in Table 3.3. Once the settings have been stored, the system powers down and waits for its initial start time to arrive. If there is a problem with the startup configuration, it can be reset by unplugging the power from the daq system for at least fifteen seconds. This resets the Watchdog timer. Upon reconnection of power the system will run in its default data collection mode unless a serial console is running and a user selects the normal state as described above. The user will then need to rerun the setup program.

The serial console mode is established by first, installing the serial cable between the buoy and a remote computer, second, running a networking software package on the remote 


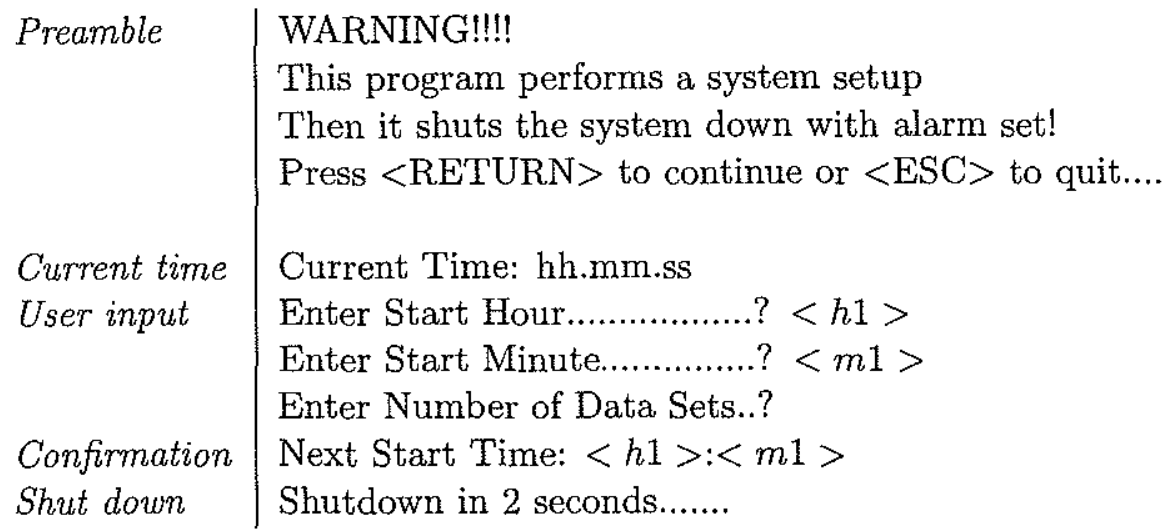

Table 3.3: Setup Configuration and User Prompted Information

computer which allows serial connections, and third, powering up the daq system. The serial cable is a 4-conductor cable which plugs into a water-tight connector on the daq housing endcap and the serial port on the remote computer. The networking software package must be configured as described in Table 3.4. HyperTerminal made by Hilgraeve works well as a simple serial console interface program for this purpose. It should be noted that only programs on the daq system which can be spooled through the serial port can be run on the remote computer when operating in serial mode. For instance, the text editor edit.com cannot be run in serial console mode, but the text editor me.exe (Micro Emacs) can be.

\section{Data Download}

The data stored on the wave gage buoy hard drive can be easily downloaded to another computer without having to open the daq aluminum housing. This is accomplished by using the following procedure:

1. Connect a $50 \Omega$ BNC (10Base-2) cable between the data acquisition endcap and the

\begin{tabular}{|rl|}
\hline Baud Rate: & 9600 \\
Data Bits: & 8 \\
Parity: & None \\
Stop Bit: & 1 \\
Flow Control: & None \\
\hline \hline Terminal Emulation: & ANSI \\
\hline
\end{tabular}

Table 3.4: Serial Console Configuration 
downloading computer network port with $50 \Omega$ terminations at each end

2. Start a serial console mode as described above

3. Change directories to $\mathrm{C}: \backslash$ data on the buoy computer

4. Run the startnet batch file on the buoy computer

5. Run the ftpsrv program on the buoy computer

6. ftp to 128.128 .93 .22 (the buoy IP address) from the downloading computer ${ }^{1}$

7. Set the transfer mode to binary

8. Download the appropriate data files and exit ftp when finished

9. Exit the ftpsrv program on the buoy by typing ' $q$ ' at the buoy computer prompt

10. Restart the buoy computer before using it to collect data again (this clears the network settings from the memory, to enable subsequent data acquisition)

\subsubsection{Basic Commands, Calibration, and File Formats}

\section{Basic Commands}

The data collection mode of operation is entirely controlled through the wave.c program. This program has various command line options which are detailed in Table 3.5. An appropriate use of the command line options for testing purposes would be

$$
\text { wave }-b 100-s
$$

which starts collecting $100 * 5.12=512$ seconds of data (51200 data samples per channel) immediately and does not shut down at the end of the data collection. When planning to use the power saving mode which shuts the system down and then wakes it up at a later time, the run_wave . bat program along with the setup.c programs must be used instead of the basic command line call (as described in the Startup section). It should be noted that

\footnotetext{
${ }^{1}$ The downloading computer must be configured with a $128.128 . *$.* IP address or the buoy computer will not recognize it
} 


$\begin{array}{lll}-\mathrm{g}<\text { gain }> & \text { Set A/D board gain } & \text { (default }=1 \text { ) } \\ -\mathrm{i}<\text { interval } & \text { Set time between beginning of data sets (in min.) } & \text { (default }=15 \text { ) } \\ -\mathrm{b}<\text { nblks }> & \text { Set number of 5.12 sec. data blocks to collect } & \text { (default = 32) } \\ -\mathrm{q} & \text { Sets quiet mode (turns off debug screen displays) } & \\ -\mathrm{s} & \text { Inhibits shutdown of daq after data collection } & \\ -\mathrm{h}<\text { hour }> & \text { Set start hour to (using 24 hour clock) } & \text { (no default) } \\ -\mathrm{m}<\mathrm{minute}> & \text { Set start minute to } & \text { (no default) } \\ -\mathrm{c} & \text { Enable calibration step after first block of data } \\ -? & \text { Prints a help menu for the command line options } \\ & \text { Table 3.5: Command Line Options for wave.c }\end{array}$

while the command line options appear to accomplish the same purposes as the setup.exe program, they should generally not be used except when testing the instrument without using the power saving shut down mode. The reason for this is that each time the buoy 'wakes up' from the power save mode to collect a subsequent data set, it references the run_wave. bat batch file, NOT any original wave.exe program call that may have been entered at the $\mathrm{C}: \backslash$ prompt.

\section{Obtaining Calibration Files}

The calibration files are used in the post processing of the buoy data and should be collected both before and after a deployment while the buoy is out of the water. Their purpose is to provide information about the noise in the system, to verify that each of the channels is functioning properly, and to determine the actual transfer function for each channel. The instrument is calibrated by electronically triggering a relay, which steps the amplitude of the oscillator voltage, and then stores the response of the system to this step. The electronic triggering of the step is controlled in the wave.c program when the $-\mathrm{c}$ command line option is used. To improve the statistical validity of the calibration results, eight files are collected during a standard calibration: four calibration files and four non-calibration (noise) files. This process is automated with a batch file that repeatedly calls the wave.c program with the appropriate command line options. This batch file, calib.bat, is located in the $\mathrm{C}: \backslash \mathrm{calib}$ directory. The eight files generated have the same format and file names as the typical wave. c output files, but they are only 2 blocks (10.24 seconds) long, and they are alternately a noise file, then a calibration file, then a noise file, and so on. 


\section{File Description and Formats}

The output data files are stored on disk with month, day, hour, minute.* file names

mmddhhmm.wav - wave wire data from all 64 channels (stored in binary)

mmddhhmm.tmp - temperature data (stored in ASCII)

mmddhhmm.kvh - compass data (stored in ASCII)

The * wav file has a 64 -byte header and the data is double-multiplexed as two-byte signed integers with 8 data values per line. The data is stored in 5.12 second blocks, is indexed by board, and is also indexed by time step; the first data point (time t1) for the first 16 channels (board 1) is stored, then the second data point (time t2) for the first 16 channels is stored, and so on until 512 data points for each of the 16 channels has been stored. Each of the remaining three boards (channels 17-32, channels 33-48, and channels 49-64) is stored the in the same fashion. Once the first 512 samples of each of the 64 channels have been stored, then the cycle repeats for the second block of 512 samples. Figure 3-12 illustrates this organization. The wav2asci . exe program converts the binary $<$ run numeric $>$. wav file into a <run numeric > asc ASCII file and is described in Section 3.6.3.

The * tmp file has a 64-byte header and each data value, which has units of volts, must be multiplied by a calibration factor of $10^{\circ} \mathrm{C} /$ volt to get an output in degrees Celsius. The temperature probe value is not collected at the $100 \mathrm{~Hz}$ data collection rate of the wave wire data because it does not vary over 0.01 second time intervals, rather it varies over five to ten seconds. It is instead collected once every 5.12 seconds (after each 'block' of data). It's primary use is to monitor the temperature sensitive capacitors in the central analog electronics housing. If any significant temperature shifts (more than $5^{\circ}$ ) occur, the capacitor values in the calibration calculations can be modified.

The *.kvh file has a 64-byte header and each data value is stored in KVH format as described in Table 3.6. The compass data is not collected at $100 \mathrm{~Hz}$, because of its rather coarse resolution $\left(5^{\circ}\right)$ and because it tends to vary only every few seconds. It is, therefore, collected once every second. If the seas are fairly choppy, the compass output can be very noisy because the gimbal on which it is mounted cannot compensate for the full motion of the buoy. In these cases, tests in the lab showed that computing a running window average over 9 seconds was a fairly accurate way to reproduce the approximate compass heading of 


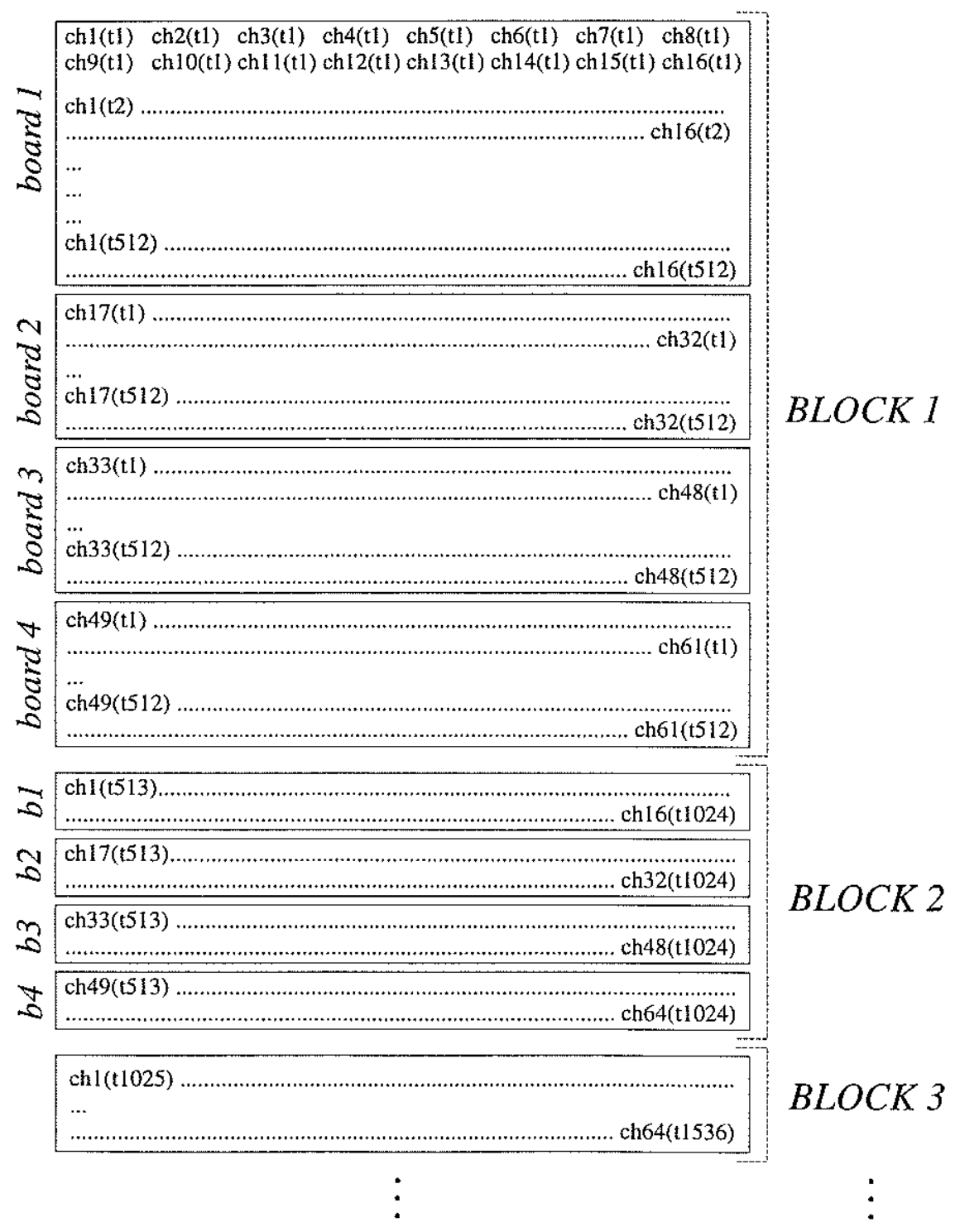

Figure 3-12: Data Format for *. wav File 
EXAMPLE DATA OUTPUT STRING: \$abc.d,e,ff*CHECKSUM<cr>

abc.d compass heading to tenth of a degree

e output mode ( $D$ if in degrees, $M$ if in mils)

ff $\quad$ status of magnetic surroundings ( $\mathrm{OL}=$ overload, $\mathrm{OK}=$ data valid)

* indicates beginning of 2-byte checksum of message

CHECKSUM hexadecimal checksum of all characters between $\$$ and $*$

Table 3.6: KVH C100 Compass Data Format

the instrument.

\subsubsection{Diagnostic Programs}

Numerous diagnostic programs were written to allow trouble shooting and testing of the wave gage buoy operation and data acquisition. They are located in the $c: \backslash$ wave directory on the wave gage buoy computer. The key programs are described below.

test_*.c Samples the 16 channels of board *, displays them, then recaptures them each time $<$ enter $>$ is hit. $<$ ESC $>$ exits.

min_max.c Using 'min_max $<$ board $(1-4)><$ channel $(0-15)>$ ' the user selects a board and channel and program returns a hexadecimal and decimal display of the minimum and maximum voltage output from that channel over a 10 second interval.

chantest.c Using 'chantest < board (1-4)><channel (0-15) >' the user selects a board and channel. The program returns a continuous screen display of the voltage output from that channel in both hexadecimal and decimal units. $<\mathrm{ESC}>$ exits.

test_64.c Outputs a running screen display of all 64 wave wire voltage outputs. $<\mathrm{ESC}>$ exits.

wav2asci.c Converts binary mmddhhmm, wav file into an ASCII file, mmddhhmm.asc, with the format described in Figure 3.6.3

battery.c Returns a continuous display of the current battery voltage. $<$ ESC $>$ or $<$ RETURN $>$ exits. 


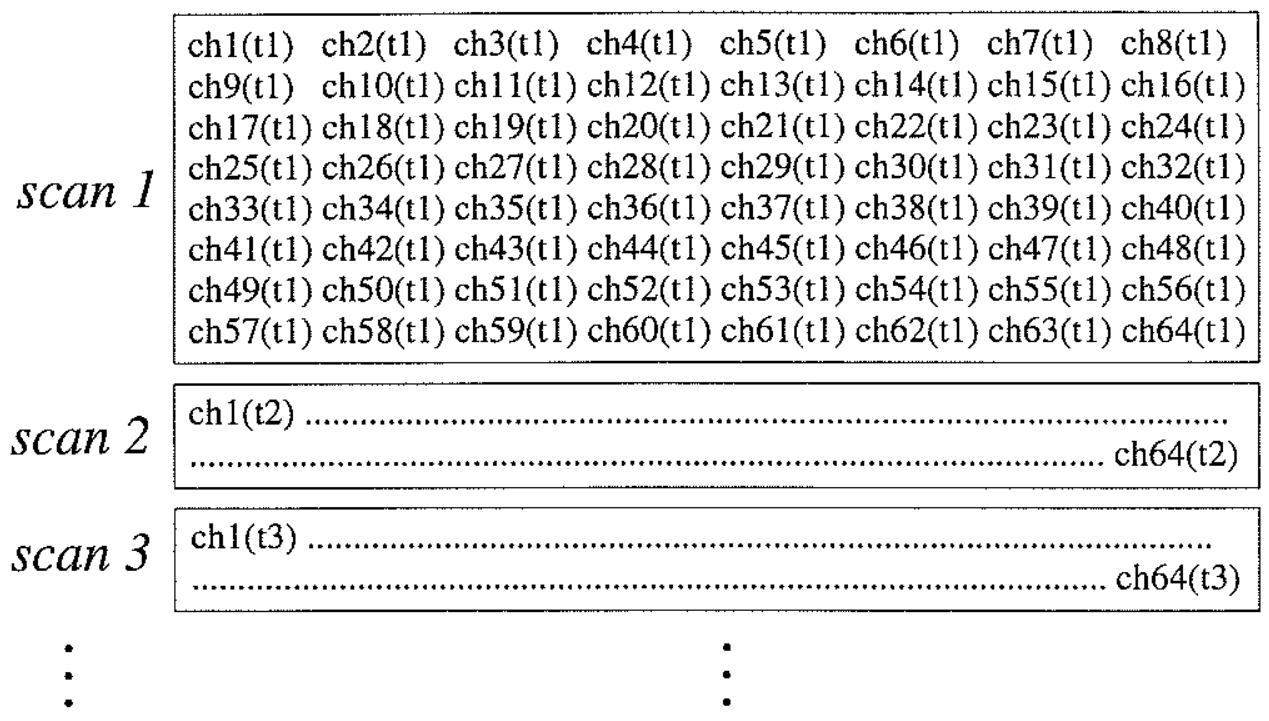

Figure 3-13: Output File Format of wav2asci.exe Program

\subsection{Data Processing}

\subsubsection{Process Description}

One of the primary difficulties in obtaining directional spectra of the sea surface is the physical difficulty in obtaining a spatially two-dimensional measurement of the sea surface elevation. The input signal must also be measured over time at every sampling point to allow temporal averaging if the spatial dimensions of the region are not expansive. One of the features of the analysis used to process the circular wire wave gage data is that evenly spaced data sampling points are not required. Instead of using an evenly spaced grid, a more modest spatial sampling is used. The following analysis describes the method used to obtain the directional spectrum of the sea surface given that the spatial two-dimensional vertical surface covariance can be calculated at some known spatial lags. 


\section{The Vertical Surface Covariance}

Let the sea surface height covariance be defined as in Equation 2.3. The covariance function is assumed to occupy a region in covariance space bounded by

$$
x^{2}+y^{2} \leq R^{2}
$$

To simplify the implementation of the spectral estimation method to be used, a rectangular (or square) region in covariance space is adopted as opposed to a circular one. To accomplish this, additional zero-valued data points have been added to data set in the corners of a box bounded by

$$
|x|<L_{x} \quad \text { and } \quad|y|<L_{y}
$$

where $r=(x, y), L_{x}$ and $L_{y}$ are the horizontal and vertical distances spanned by the spatial differences in the sample locations, and $R$ is the radius of this occupied region $\left(R=L_{x}=L_{y}\right.$ for a square region). A smoothing window is used such that $\rho(\boldsymbol{r} ; t)$, the height covariance, smoothly approaches zero at the boundaries of this region and outside the region it is zero. A smoothing window is used because the covariance function is only known within this finite circular region, and in a similar fashion that one-dimensional smoothing windows are used to reduce side lobe contamination in the Fourier transform of a finite time series records, a two-dimensional window is needed to reduce the side lobe contamination in the resulting transform of the covariance data. This is accomplished with an energy conserving window, $W$, such that given

$$
w=\frac{\sqrt{x^{2}+y^{2}}}{R}
$$

then

$$
W= \begin{cases}2 / \pi\left[\arccos (w)-w \sqrt{1-w^{2}}\right] & \text { if } w \leq 1.0 \\ 0 & \text { otherwise }\end{cases}
$$


so that

$$
\rho_{w i n}(\boldsymbol{r} ; t)=\rho(\boldsymbol{r} ; t) \cdot W
$$

and

$$
\int_{-1}^{1} W d w=1
$$

Let the windowed covariance function be represented by an infinite sum of its Fourier coefficients within the bounds of Equation 3.3 such that

$$
\rho_{w i n}((x, y) ; t)=\sum_{m=-\infty}^{\infty} \sum_{n=-\infty}^{\infty} A_{n m} \exp \left(\frac{i \pi m x}{L_{x}}\right) \exp \left(\frac{i \pi n y}{L_{y}}\right)
$$

where $A_{n m}$ are the corresponding Fourier coefficients.

Since $\rho((x, y) ; t)$ must be symmetric due to the nature of its construction, then $A_{-n-m}=$ $A_{n m}$. The individual terms of Equation 3.8 can now be written as

$$
\begin{aligned}
A_{n m} \exp \left(\frac{i \pi m x}{L_{x}}\right) \exp \left(\frac{i \pi n y}{L_{y}}\right)+A_{-n-m} \exp \left(\frac{-i \pi m x}{L_{x}}\right) & \exp \left(\frac{-i \pi n y}{L_{y}}\right)= \\
& 2 A_{n m} \cos \left(\frac{\pi m x}{L_{x}}+\frac{\pi n y}{L_{y}}\right)
\end{aligned}
$$

Expanding this and rewriting the whole summation from Equation 3.8 gives

$$
\begin{aligned}
\rho_{\text {win }}((x, y) ; t) & =\sum_{n=-\infty}^{\infty} A_{n 0} \cos \left(\frac{\pi n y}{L_{y}}\right)+\sum_{m=1}^{\infty} \sum_{n=-\infty}^{\infty} 2 A_{n m} \cos \left(\frac{\pi m x}{L_{x}}+\frac{\pi n y}{L_{y}}\right) \\
& =A_{00}+2 \sum_{n=1}^{\infty} A_{n 0} \cos \left(\frac{\pi n y}{L_{y}}\right)+\sum_{m=1}^{\infty} \sum_{n=-\infty}^{\infty} 2 A_{n m} \cos \left(\frac{\pi m x}{L_{x}}+\frac{\pi n y}{L_{y}}\right)
\end{aligned}
$$

Because the wire locations on the wave gage are fixed, the vector between any two gage elements has a known value $\left(x_{p}, y_{p}\right)$ for $p=1,2,3, \ldots, p_{\max }$. The covariance function evaluated at the location of each of these vectors is approximated as the time average of the products of the surface elevations at the two corresponding gage elements, as per its definition. Since only a finite set of measured surface elevation data is available, discrete 
values of the covariance function, $\rho\left(\left(x_{p}, y_{p}\right) ; t\right)$, can be calculated from the finite set of measured surface elevation data. The series from Equation 3.10 can then be approximated by its truncated form:

$$
\begin{aligned}
\rho_{w i n}\left(\left(x_{p}, y_{p}\right) ; t\right) \simeq A_{00}+2 \sum_{n=1}^{N-1} A_{n 0} \cos \left(\frac{\pi n y_{p}}{L_{y}}\right) & \\
& +2 \sum_{m=1}^{M-1} \sum_{n=-(N-1)}^{N-1} A_{n m} \cos \left(\frac{\pi m x_{p}}{L_{x}}+\frac{\pi n y_{p}}{L_{y}}\right)
\end{aligned}
$$

The unknowns in this equation are the Fourier coefficients, which are the $A_{n m}$ s. There are $M(2 N-1)-N+1$ of these unknown coefficients. If enough (well behaved) values of the covariance are known, this system of equations can be solved for the $A_{n m}$ s. In other words, if

$$
p_{\max }>M(2 N-1)-N+1
$$

then one can use a minimum mean square error matrix solver technique to find the Fourier coefficients of the covariance matrix by solving a matrix equation of the form

$$
\left[\overline{\rho_{w i n}\left(x_{p}, y_{p}\right)}\right]=\left[A_{n m}\right]\left[C \cos \left(\frac{\pi m x_{p}}{L_{x}}+\frac{\pi n y_{p}}{L_{y}}\right)\right]
$$

where $C$ is a set of constant coefficients determined by putting Equation 3.11 in this matrix form.

\section{The Directional Spectrum}

The classical method of determining a two-dimensional spectrum of a spatially two-dimensional signal is to take the two-dimensional Fourier transform of the covariance of that signal. For a continuous signal this corresponds to computing the following integral

$$
\Phi\left(k_{x}, k_{y}\right)=\frac{1}{(2 \pi)^{2}} \int_{-\infty}^{\infty} \int_{-\infty}^{\infty} \rho(x, y) \exp \left[-i\left(k_{x} x+k_{y} y\right)\right] d x d y
$$


If $\rho(x, y)$ is replaced by its representation from Equation 3.8 and then the exponential terms are simplified, the following form for the two-dimensional spectrum results

$\Phi\left(k_{x}, k_{y}\right)=\frac{1}{(2 \pi)^{2}} \sum_{m=-\infty}^{\infty} \sum_{n=-\infty}^{\infty} \int_{-L_{y}}^{L_{y}} \int_{-L_{x}}^{L_{x}} A_{n m} \exp \left[i x\left(\frac{\pi m}{L_{x}}-k_{x}\right)\right] \exp \left[i y\left(\frac{\pi n}{L_{y}}-k_{y}\right)\right] d x d y$

The integrals can now be separated and individually evaluated giving

$$
\Phi\left(k_{x}, k_{y}\right)=\frac{1}{(2 \pi)^{2}} \sum_{m=-\infty}^{\infty} \sum_{n=-\infty}^{\infty} A_{n m} \frac{2 \sin \left[L_{x}\left(\frac{\pi m}{L_{x}}-k_{x}\right)\right]}{\left(\frac{\pi m}{L_{x}}-k_{x}\right)} \frac{2 \sin \left[L_{y}\left(\frac{\pi n}{L_{y}}-k_{y}\right)\right]}{\left(\frac{\pi n}{L_{y}}-k_{y}\right)}
$$

Simplifying this slightly gives the final result of

$$
\Phi\left(k_{x}, k_{y}\right)=\frac{4}{(2 \pi)^{2}} \sum_{m=-\infty}^{\infty} \sum_{n=-\infty}^{\infty} A_{n m} \frac{\sin \left(\pi m-L_{x} k_{x}\right)}{\left(\pi m-L_{x} k_{x}\right)} \frac{\sin \left(\pi n-L_{y} k_{y}\right)}{\left(\pi n-L_{y} k_{y}\right)}
$$

This equation can be used to calculate the spectrum at any wave number values, $\left(k_{x}, k_{y}\right)$, assuming the $A_{n m}$ coefficients are known. For very particular values of $k_{x}$ and $k_{y}$ the formula above simplifies even further. This is for:

$$
\begin{aligned}
& k_{x}=\frac{\pi m^{\prime}}{L_{x}}, \\
& \quad \Phi\left(k_{x}, k_{y}\right)=\frac{4}{(2 \pi)^{2}} \sum_{n=-\infty}^{\infty} A_{n m^{\prime}} L_{x} L_{y} \frac{\sin \left(\pi n-L_{y} k_{y}\right)}{\left(\pi n-L_{y} k_{y}\right)}
\end{aligned}
$$

$$
\begin{aligned}
& \text { and for } k_{y}=\frac{\pi n^{\prime}}{L_{y}} \text {, } \\
& \qquad \Phi\left(k_{x}, k_{y}\right)=\frac{4}{(2 \pi)^{2}} \sum_{m=-\infty}^{\infty} A_{n^{\prime} m} L_{x} L_{y} \frac{\sin \left(\pi m-L_{x} k_{x}\right)}{\left(\pi m-L_{x} k_{x}\right)}
\end{aligned}
$$

Finally,

$$
\text { if } \begin{aligned}
k_{x}=\frac{\pi m^{\prime}}{L_{x}} & \text { and } k_{y}=\frac{\pi n^{\prime}}{L_{y}} \text { then } \\
\Phi\left(k_{x}, k_{y}\right) & =\frac{4}{(2 \pi)^{2}} A_{n^{\prime} m^{\prime}} L_{x} L_{y}
\end{aligned}
$$


From Equation 3.11 the $A_{n m}$ coefficients can be calculated for $m=1,2, \ldots, M-1$ and $n=-(N-1),-(N-1)+1, \ldots, 0, \ldots,(N-1)$.

The equations above provide the key analytical tools for both estimating the covariance function given a finite set of surface height measurements, and evaluating the twodimensional spectrum at either specific wavenumber locations or at any location, given a Fourier coefficient expansion of the covariance function. The limitations of this method stem primarily from the accuracy of the covariance estimate in Equation 3.11; if there are not enough values of the covariance function or the minimum mean square error fit is not well behaved, the resulting Fourier coefficients will not be good estimates, leading to inaccurate results using Equations 3.17 through 3.20. Every effort has been made to assure that these errors are as small as possible. Some tests to determine the accuracy of the analysis methods were described in the author's Masters thesis [61].

\section{Calibration}

If both the input and output of a linear system are known in time, they can be transformed into the frequency domain and used to generate the frequency response function as described in Section 3.3.2. Once the frequency response has been calculated, it can be used with the system output to reconstruct the input signal - this is how the signal outputs from the wave gage can be used to regenerate the measured wave heights. The goal of calibration is to produce the frequency response.

For any analog electronic system like the wave gage, the shape of the frequency response function can be modeled using the nominal values of the various circuit elements that make up the frequency-dependent portions of the analog filtering. This was illustrated in Table 3.1. This model can predict the shape of the frequency response, but it cannot accurately predict the magnitude or the exact location of the peak of the frequency response. The reason for this is that the values of the circuit elements have some uncertainty associated

with them. For instance, capacitors are generally purchased with specific tolerances such as 5 or $10 \%$. The exact values of all the circuit elements are not known unless measured independently. More important, the values of these elements can and do change with temperature. The modeled theoretical frequency response cannot, therefore, be used alone 
to represent the precise characteristics of the system.

Tests were conducted to measure the temperature dependency by putting the analog electronics in a temperature-controlled oven. The resulting affects on the calibration of each wire as a function of temperature are used during the calibration of the instrument if there is a significant temperature change over the course of the experiment.

The frequency response of the filtering system can most easily be measured by taking the Fourier transform of the time series output of the system's response to an impulse input signal. Unfortunately, generating a 'real-world' impulse response is rather difficult, and due to the nature of the circuitry, it would be difficult to insert the impulse into the filter input if one could be generated. Generating a step input in the system by stepping the amplitude of the high-frequency carrier wave is much easier to accomplish and contains the relevant information that an impulse response would contain. This relationship between Fourier transform of the step response, $Y_{\text {step }}(\omega)$, and the transfer function, $H(\omega)$, is

$$
H(\omega)=Y_{\text {step }}(\omega) \cdot \frac{i \omega}{h}
$$

where $h$ is the effective length in centimeters of nichrome wire that is shorted out to generate the step which is a predetermined constant.

Now that the frequency response for each channel has been both measured and modeled, all that remains is to fit the model to the actual measurement to establish a 'best-fit' model. This new model will be used to 'unfilter' the output signal from each of the channels and return the wave height. The reason a fitted model must be used instead of simply using the actual measured frequency response is because the measured frequency response only contains magnitude information, but the actual frequency response contains phase information as well. The phase information is tied up in the temporal relationship between the step and its response. This temporal coupling is not well measured. A fitting routine is used instead which adjusts the nominal values for the capacitors and resistors in the transfer function model until a best fit between the resulting shape and the measured shape is found. 


\subsubsection{Program Implementation}

Each analysis step in the data processing package section of Figure 3-1 is performed by a separate program. This was done because Matlab, FORTRAN, and C coding are each used to accomplish various analytical operations, to facilitate debugging and testing, and because the programs are each very lengthy both in file size and in processing time. The following sections contain brief descriptions of each of the analysis programs. These descriptions are not meant to expand on the discussion of the signal processing and calibration methods presented earlier in this Chapter and in Chapter 2, but are instead meant as a guide both to the use of the code and to the implementation of the theory.

\section{Calibration (step 1): calib.m}

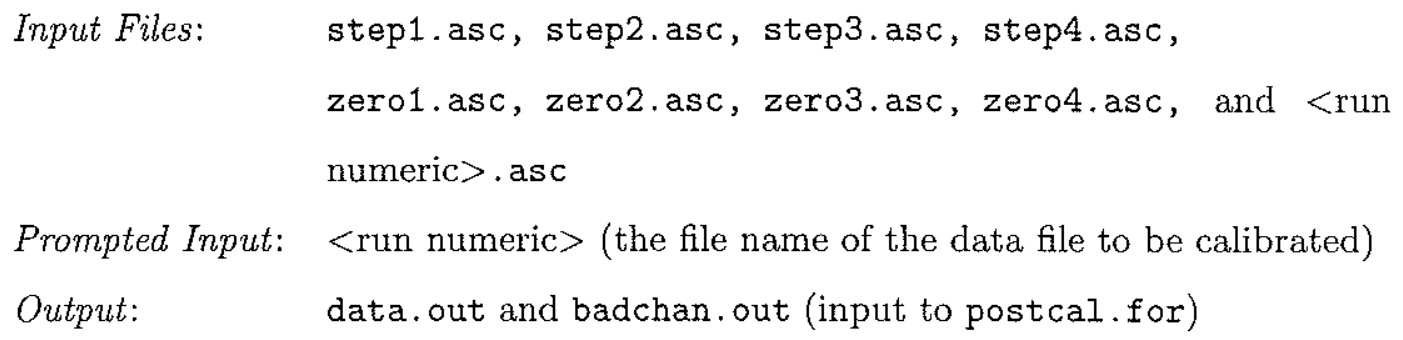

Prompted Input: <run numeric> (the file name of the data file to be calibrated)

Output: data.out and badchan.out (input to postcal.for)

To calibrate the wave gage buoy output a fairly detailed set of steps must be followed:

1. Convert the <run numeric > wav file into an ASCII file using wav2asci.c.

2. Convert the 8 calibration files from binary into ASCII files using wav2asci.c.

3. Rename the files containing step information to step1.asc, step2.asc, step3.asc, and step4.asc, and rename the files containing noise information to zero1.asc, zero2.asc, zero3.asc, and zero4.asc.

4. Copy the step* and zero* files into a directory with the calib.m file.

5. Open Matlab in this directory and run the calib.m Matlab file. 


\subsubsection{Calibration (step 2): postcal.for}

$\begin{array}{ll}\text { Input Files: } & \text { data.out, badchan.out (output from calib.m), and } \\ & \text { <run numeric >.KVH } \\ \text { Prompted Input: } & \text { <run numeric> (the file name of the data file to be calibrated) } \\ \text { Output: } & \text { direct.out, calstats.out, stats.out, } \\ & \text { ch*spec.out, mssstats.dat, newchan*.out, and autoin.dat } \\ & \text { (input to sw_auto.c) }\end{array}$

These programs calibrate the wave wire voltage output and return wave height output at each wire location as a function of time. This is done by comparing the measured impulse response of each of the channels with a theoretical one generated as was described in Section 3.7. The theoretical impulse response is then 'tuned' so that it best matches the calibration data collected. The uncalibrated wave wire data is then divided by the 'tuned' impulse response in the frequency domain for every channel and then it is converted back into the time domain. The resulting time series have effectively had the analog pre-whitening filter backed out of them.

This calibration program also incorporates the compass information by reassigning the channel 1 time series to be the time series of the wire which is closest to magnetic North at each scan. Each of the other channels is reassigned in a corresponding manner relative to channel 1. This allows an absolute directional spectrum to be calculated relative to magnetic North. There is a little over 5 degrees of error in the resulting directional calculations due to the 5.6 degree spacing between each of the wires.

Finally, if any of the wires is determined to be broken or behaving badly, the program interpolates the wave heights between the two adjacent channels. If more than two adjacent channels are determined to be broken the data set is not used. This was not a problem for the data sets presented later in this thesis; only 4 channels needed to be interpolated.

\subsubsection{Calculation of Covariance: correl.c}

Input Files: autoin.dat (output of autocal*.for)

Prompted Input: number of scans to evaluate

Output: $\quad$ correl.mas (input to dfsum*.for) 
This program calculates the covariance of the sea-surface elevation data. This is done by multiplying each data point of a particular scan by every other data point in that scan. Each of these covariance values is a function of the difference in locations of each of the data points used to generate it. The ensemble average of the covariance of each of the 4096 scans is then calculated and printed to a file. Unlike a covariance calculated over time or in one dimension, the covariance calculated in this program is a function of translation in two dimensions, $\mathrm{x}$ and $\mathrm{y}$. A more explicit description of this program is contained in Chen's Masters thesis[12] where it is called sw_auto.c.

\subsubsection{Calculation of Directional Spectrum: dfsum*.for}

Input files: correl.mas (output of correl.c)

Prompted Input: none

Output Files: s.tec, z.tec, sk.tec, anglspec.out, ptspec.out

This program calculates the directional spectrum of an input data set which contains the magnitude of the covariance of the data set and the location at which these covariance values are known. First the covariance data set is convolved with a smoothing window, then the truncated Fourier series coefficients are calculated for the covariance data set, and finally, these Fourier coefficients are used to estimate the directional spectrum at particular wavenumbers in both the North-South and East-West direction. This method is fully described in the author's Masters thesis [61].

In addition to generating the directional spectrum, this program also produces an angle spectrum and a point spectrum. The angle spectrum is a representation of the spectrum along various slices taken along a particular radial direction from the origin. The point spectrum is calculated by integrating the two-dimensional spectrum over a particular wavenumber. These are used to compare the results of the spectral calculations to other experimentally or theoretically generated spectra data collected by other researchers. 


\section{Chapter 4}

\section{Experimental Results}

The at-sea experiment which produced the bulk of the data presented in this document was conducted on October 28, 1998 with the aid of Sam Tolkoff, Monika Bang-Campbell, and Dave Olmstead, the captain of the Woods Hole Oceanographic Institution's R/V Asterias.

\subsection{Buzzards Bay Tower Experiment}

The Buzzards Bay Tower (BUZM3) is located in the southwest entrance to Buzzards Bay, east of Newport, Rhode Island at $41.40 \mathrm{~N}$ latitude, $71.03 \mathrm{~W}$ longitude. It is a triangular C-MAN station which is monitored by the National Oceanographic and Atmospheric Administration (NOAA) and the Woods Hole Coast Guard Aids to Navigation Team. The tower is approximately 25 meters high with two platforms - the lower platform is approximately 2 meters above the mean high water mark with a lower docking level to allow easy access by boat, and the upper platform is approximately 15 meters above mean sea level and is where the bulk of the NOAA instrumentation is located as illustrated in Figure 4-1.

\subsubsection{Experimental Setup}

The instruments were mounted on the lower platform in the positions depicted in Figures 4-2 and 4-3. The circular wire wave gage buoy was deployed just East of the tower. 


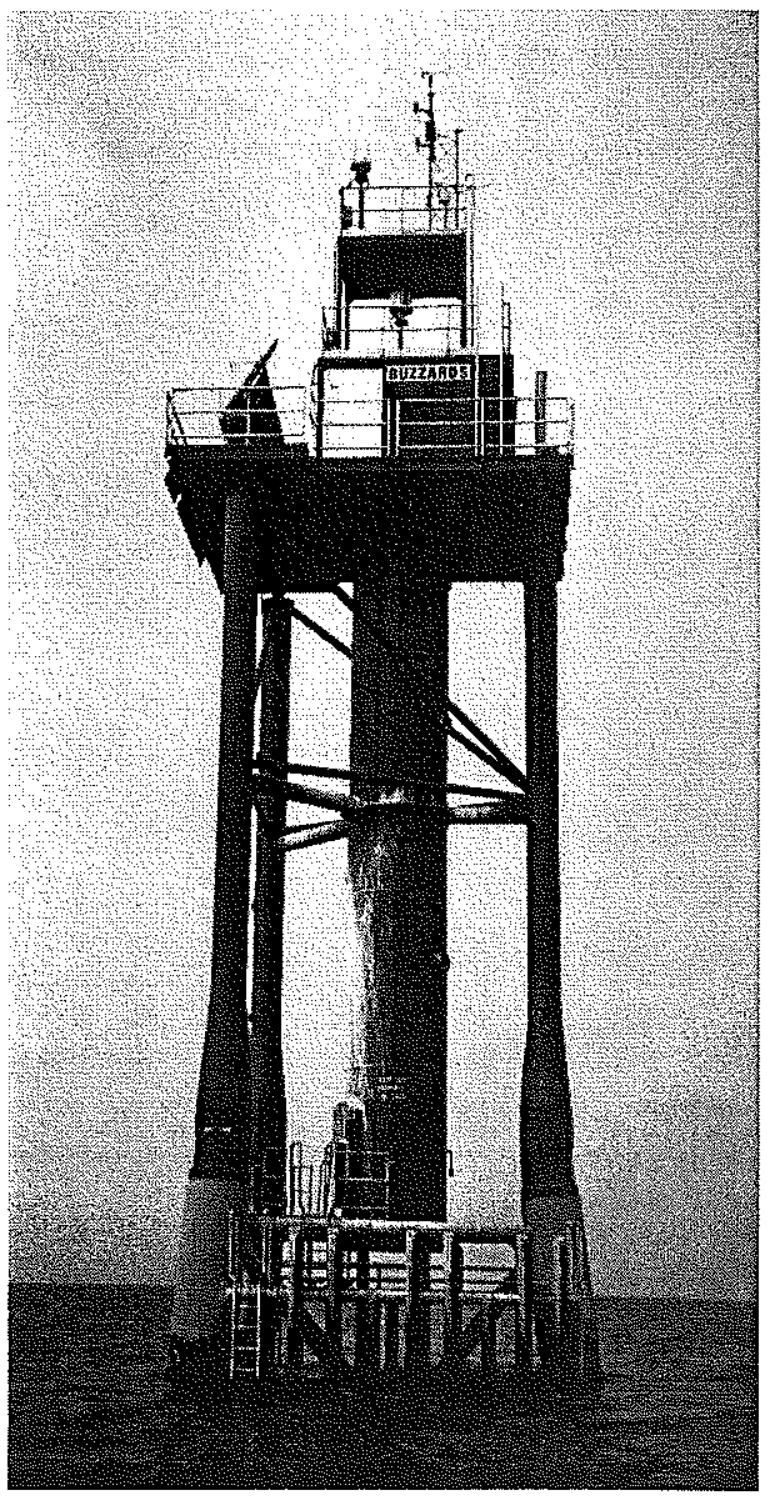

Figure 4-1: Buzzard's Bay Tower 


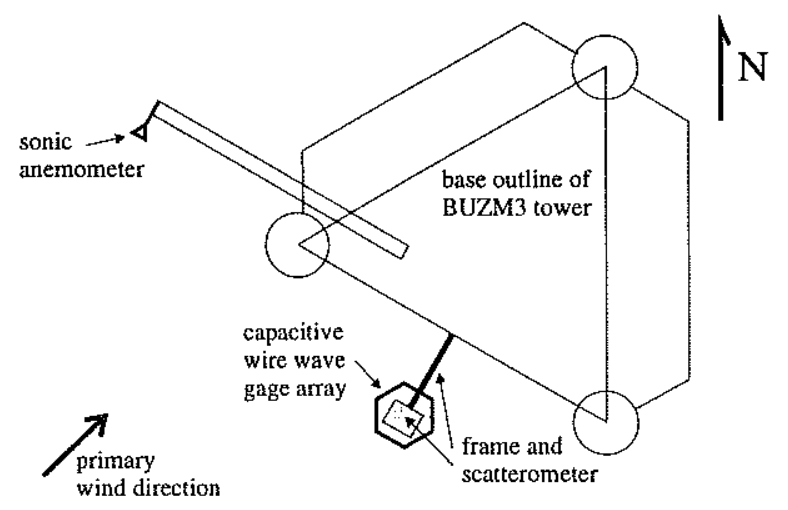

Figure 4-2: BUZM3 Instrument Layout, Top View (not to scale)

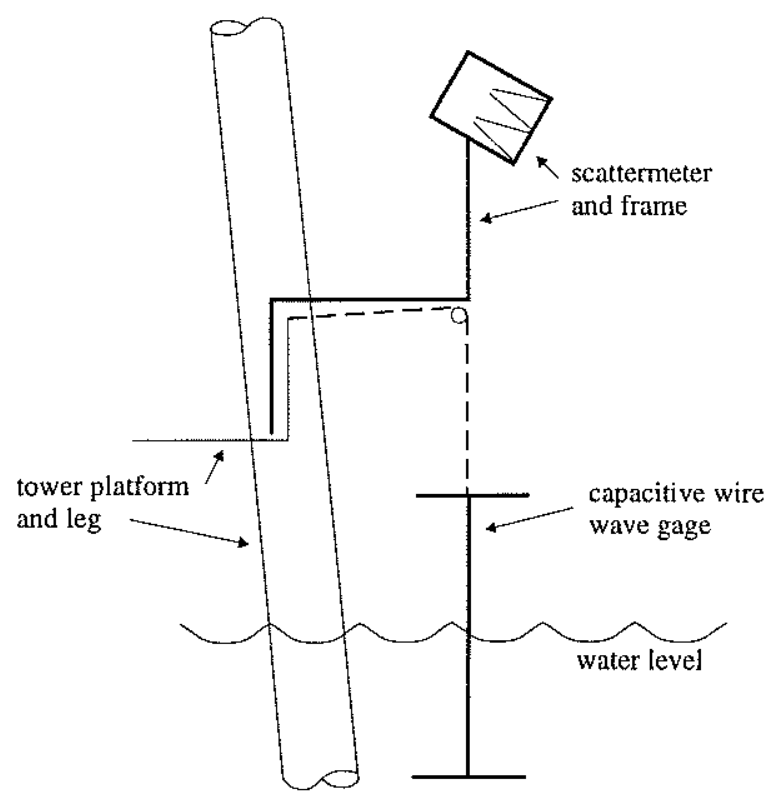

Figure 4-3: BUZM3 Instrument Layout, Side View (not to scale) 


\section{Circular Wire Wave Gage}

The circular wire wave gage was designed to float freely with the surface waves and currents to reduce flow distortion at the wire locations central to the buoy frame. For this experiment it was deployed outside of the expected wind and wave shadow of the tower and allowed to drift for the duration of the experiment. The R/V Asterias occasionally approached the buoy from down wind to obtain a Global Positioning System (GPS) fix. The drift path of the buoy is shown in Figure 4-4. Because the wind was blowing from the open ocean out of the southwest it is assumed that the conditions at the buoy were statistically similar to those experienced at the tower and that the fetch was over 100 kilometers. The similarity of surface wave conditions is important for the comparisons made between measurements collected at the tower and those collected by the buoy. The buoy drifted approximately 2.5 nautical miles over the course of its deployment, however, and the validity of even meteorological similar conditions between the buoy and the tower at these distances could be cited as a possible reason for the discrepancies between the resulting measurements at the two locations.

The buoy was deployed at 1220 EST and was preprogrammed to collect 6 data sets of 293 blocks each ( $\sim 25$ minutes) at 30 minute intervals beginning at 1215 EST. The buoy was recovered at $1506 \mathrm{EST}$. The first and last data sets were not used because the buoy was not in the water and free of the deployment vessel for their entirety.

\section{Scatterometer}

The scatterometer is a dual-polarized continuous-wave X-band Doppler backscatterometer designed and built at the Johns Hopkins University Applied Physics Laboratory and was on loan to WHOI. It has four antennas, two of which transmit the $\mathrm{H}$ - and V-polarized fields at $10.00 \mathrm{GHz}$ and $10.06 \mathrm{GHz}$, respectively, and two which receive the backscattered signals. The output from the scatterometer is a time series of in-phase and quadrature signals in both $\mathrm{HH}$ and VV polarizations (i.e. an $I$ and a $Q$ output for each polarization). The output signals were digitized at $1000 \mathrm{~Hz}$.

The scatterometer was mounted on an L-shaped frame which was bolted to a stand just above the railing of the lower platform of the tower. The frame extended horizontally 


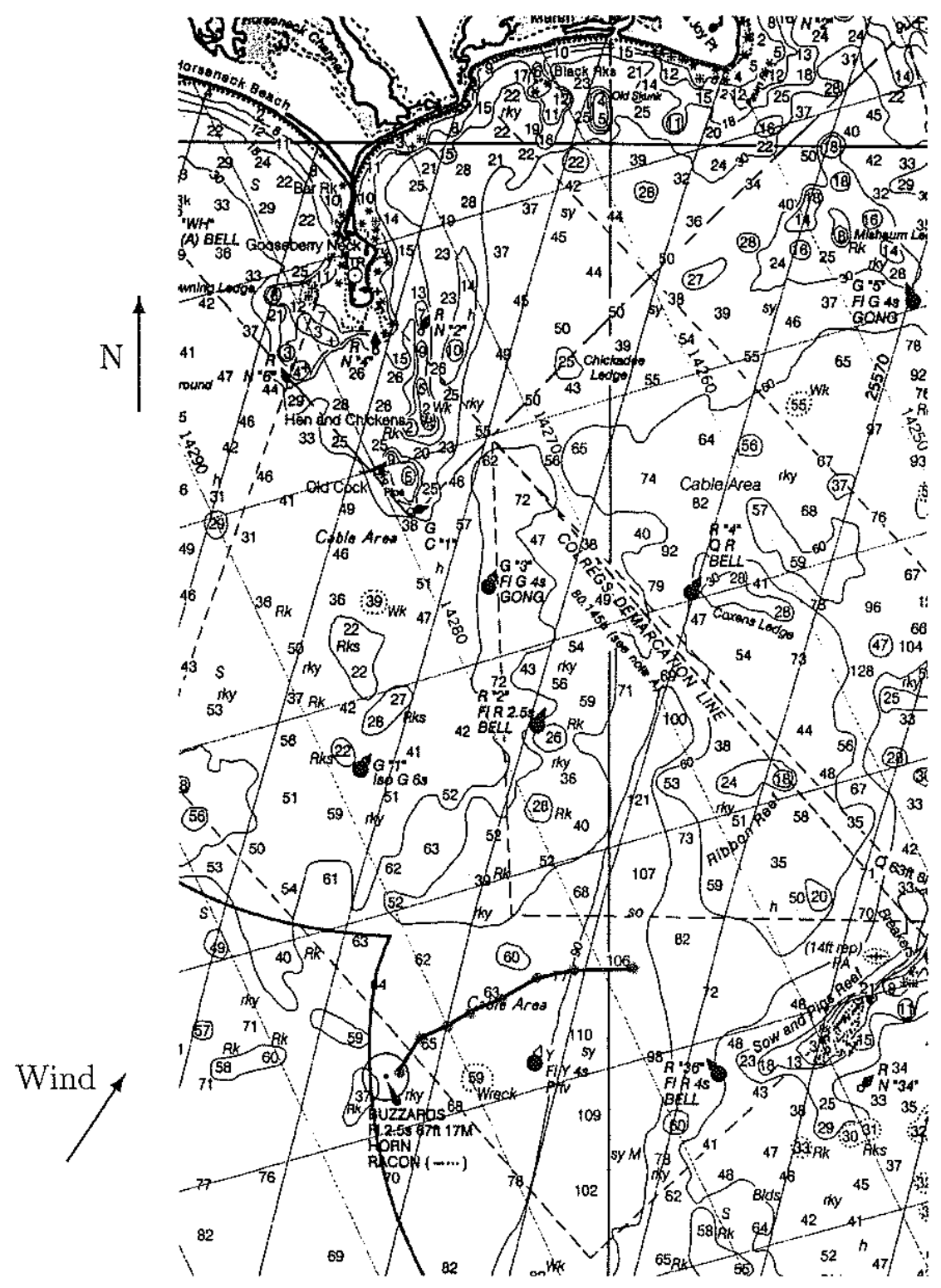

Figure 4-4: Circular Wire Wave Gage Buoy Drift Track. The Buzzards Bay Tower is located in the bottom left corner of the map. The gray dots with the thick line connecting them beginning just to the East of the Tower represent the drift track of the buoy. The gray dots represent the location fixes obtained by the Asterias during deployment. For reference purposes True North is vertically upwards and the entire image is approximately 5.5 nautical miles wide in the $\mathrm{E}-\mathrm{W}$ direction. The depths given on the chart are in feet. 
outward 1.47 meters from the railing and 1.27 meters above the railing. The railing was approximately 1 meter high. Three fiber lines were used to further secure the frame - two extended to either side of the frame and were tied to the railing, the third was secured vertically above the frame. The frame also allowed the scatterometer to be mounted at a user specified angle of declination - an incidence angle of $59^{\circ}$ was used for this experiment. Additionally, the very top portion of the frame housed a motor-driven rotary mechanism which pivoted the scatterometer in an arc of more than $180^{\circ}$, allowing a field of view encompassing the entire windward face of the tower. The scatterometer completed one $180^{\circ}+$ rotation in approximately 28 seconds. At each end of the rotation a limit switch was triggered which reversed the direction of the rotation. An additional $15^{\circ}$ of rotation at each end of the arc allowed enough extra time for the small motion caused by the abrupt change in direction of the scatterometer to be adequately damped, so as to not influence the scatterometer's measurements during the $180^{\circ}$ arc when it was actually viewing the open water and not the tower.

At $1346 \mathrm{EST}$ on the day of the experiment, the mean water level at the tower was approximately 2 meters below the grating on the lower platform. With this water height and the dimensions of the frame given above, the center of the field of view of the scatterometer was just over 7 meters from the tower and the approximate Bragg wavelength was 1.7 centimeters. The distance of the scatterometer footprint was maximized to ensure that any waves reflected from the tower influenced the scattered return as little as possible.

The scatterometer data shown later in this chapter is not presented in calibrated $\sigma_{0}$ form. Because the raw backscattered power is directly proportional to $\sigma_{0}$ through a constant calibration factor, and the only aspect of the scattered return of significance to this study is its azimuthal shape (a purely relative quantity), it was not necessary to absolutely calibrate the instrument.

\section{Capacitive Wire Wave Gage}

A capacitive wave gage array with six wires was hung from the frame on which the scatterometer was mounted. The capacitive wires were constructed following the guidelines described by Chapman and Monaldo [10], the electronics were designed by Andrey Karach- 
intsev at the University of Rhode Island (URI), and the processing code used to generate two dimensional spectra was written by Kurt Hanson [23], also from URI. The instrument operates by measuring the capacitance between the insulated core of the wave wire, and the conductive salt water surrounding it. Each instrument outputs voltages that are digitized at $1000 \mathrm{~Hz}$. Calibration measurements are then used to relate the voltage level to the appropriate capacitance value. The calibration tables for the six wires used are given in Appendix I. The resulting capacitance values can be related to the level of the salt water surrounding the wire and, thus, be used to determine sea surface height.

The wires were distributed unevenly around a hexagonal frame with a minimum spacing between wires of 0.3 meters and a maximum spacing of 0.97 meters as pictured in Figure 45. The upper and lower hexagonal frames which supported the electronics and 1.6 meter long wires were separated by a 1.8 meter long thin vertical pole located in the center of the hexagon. Changes in the mean water level due to the tides required that the frame occasionally be moved vertically in order to insure that the measured waves not be clipped by limited vertical range of the wires.

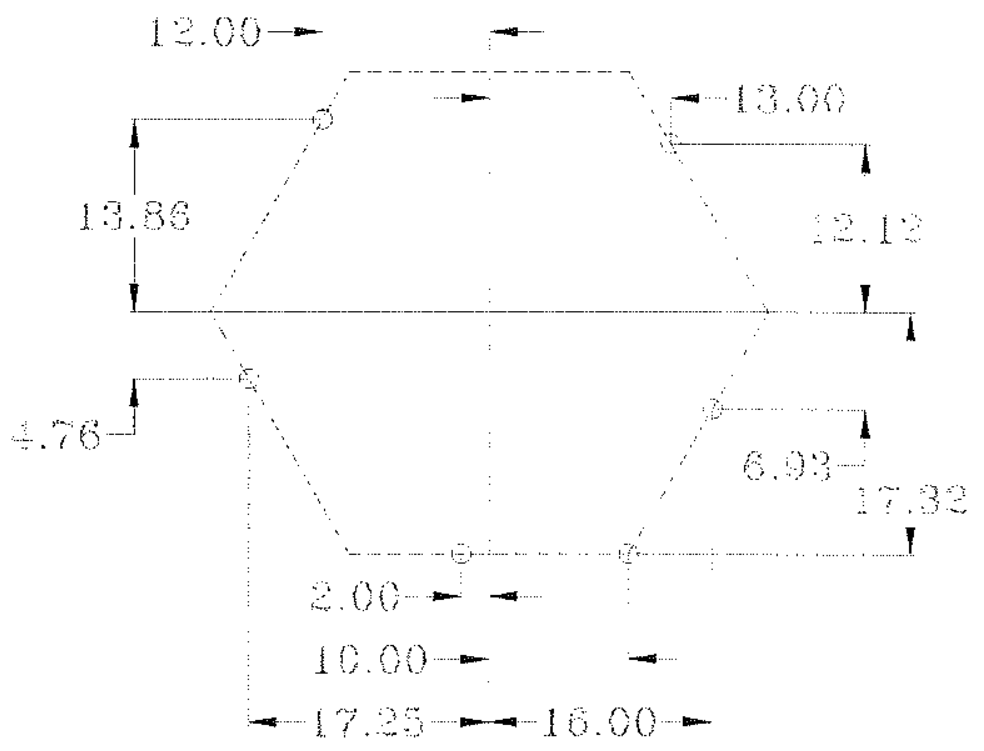

Figure 4-5: Capacitive Wire Wave Gage Hexagonal Frame and Wire Location Diagram. Dimensions are in inches. The hexagonal frame is shown with a dashed line and the wire locations are indicated with open circles 
The top of the array was secured to the scatterometer frame with a pulley and rope and was also tied to the railings to minimize twisting. Because the lower hexagonal frame was not attached to anything, it tilted and rotated slightly with the passing of the larger waves. It was estimated by observation that errors caused by these unavoidable motions were not greater than 2 centimeters in wave height and $10^{\circ}$ in angle.

\section{Sonic Anemometer}

A CSAT3 Campbell Scientific Inc. (Logan, Utah) three-dimensional sonic anemometer was mounted on a triangular antenna frame and extended about 2.6 meters away from the tower base. The boom was extended parallel to the face on which the scatterometer was mounted, and the sensor was turned into the wind to allow for minimum flow distortion as illustrated in Figure 4-2. The location of the sensor was approximately 2.6 meters above the mean water surface. The boom was secured from above with a line which, along with the bolting of the boom to the tower platform, minimized the motion of the sensor. The analog outputs from the sonic anemometer were calibrated using the low range calibration constants provided by the manufacturer in the Campbell Scientific CSAT3 Manual, page 7, and shown in Table 4.1 .

$$
\begin{array}{cccc} 
& u_{x} & u_{y} & u_{z} \\
\text { Slope }\left(\mathrm{m} \mathrm{s}^{-1} \mathrm{~V}^{-1}\right): & 6.5536 & 6.5536 & 1.6384
\end{array}
$$

Table 4.1: Sonic Anemometer Calibration Coefficients

\subsubsection{Buzzards Bay Tower NOAA Data}

The NOAA instrumentation on the BUZM3 C-MAN tower includes the following measurements: wind direction, wind speed, wind gust magnitude, air temperature, and barometric pressure. The anemometer is located 24.8 meters above mean sea level, the air temperature sensor is located 24.5 meters above mean sea level, and the barometer is located 17.4 meters above mean sea level. The other sensors at the tower were either broken or not reliable as recorded at the National Data Buoy Center station information web page on October 28th, 
1998. For further information on the tower and its instrument suite, please reference either the National Weather Service web site http://www.nws.fsu.edu or the National Data Buoy Center web site http://www.ndbc.noaa.gov. Both web sites contain similar information and allow access to archived data.

\subsubsection{Results}

\section{General Ocean and Atmospheric Conditions}

The atmospheric conditions during the experiment on October 28th were fluctuating. The barometer was dropping, the air temperature was rising, and the wind velocity was slowly increasing. These trends can be seen in Figures 4-6 and 4-7. ${ }^{1}$ They are shown for the sole purpose of establishing the synoptic conditions during the experiment. The more detailed wind velocity and direction information shown in Figures 4-8, 4-9, and 4-10 2 is used for the spectral model input parameters used in Chapter 5. A histogram showing the distribution of the wind direction measurements (unaveraged) during the first data set (10281315) is also included in Figure 4-11. Histograms of the other two wind direction data sets show a similar shape. Over the course of the experiment the location of the peak of the wave height frequency spectrum remained constant at approximately $0.30 \mathrm{~Hz}$ as measured by time series spectral calculations using the capacitive wire wave gage data.

The long wave properties were measured using the capacitive wire wave gage. Each wire was sampled at $1000 \mathrm{~Hz}$, but the instrument's maximum resolution is only about $4 \mathrm{~Hz}$ due to electronic and wire limitations, so the data was subsampled down to $100 \mathrm{~Hz}$. This lower frequency is still oversampled, but it prevented any high frequency signal or noise aliasing which can be verified by comparing a single channel frequency spectrum from both the original and the subsampled data sets shown in Figure 4-12. Figure 4-13 shows an average of the one-dimensional frequency spectra from each of the six wires for each data set. These three plots show a consistency both in the location of the peak of the spectrum as well as

\footnotetext{
${ }^{1}$ All azimuthal experimental measurements in this thesis are given in Magnetic North coordinates, except where noted, and will be referred to simply as North. At the time of this experiment the variation between Magnetic North and True North was $15^{\circ} 31^{\prime} \mathrm{W}$.

${ }^{2}$ The wind velocity data shown in Figures 4-8, 4-9, and 4-10 was adjusted from its measurement height of 2.6 meters to a standard 10 meter height using the Charnock model and a neutral stability assumption [11].
} 

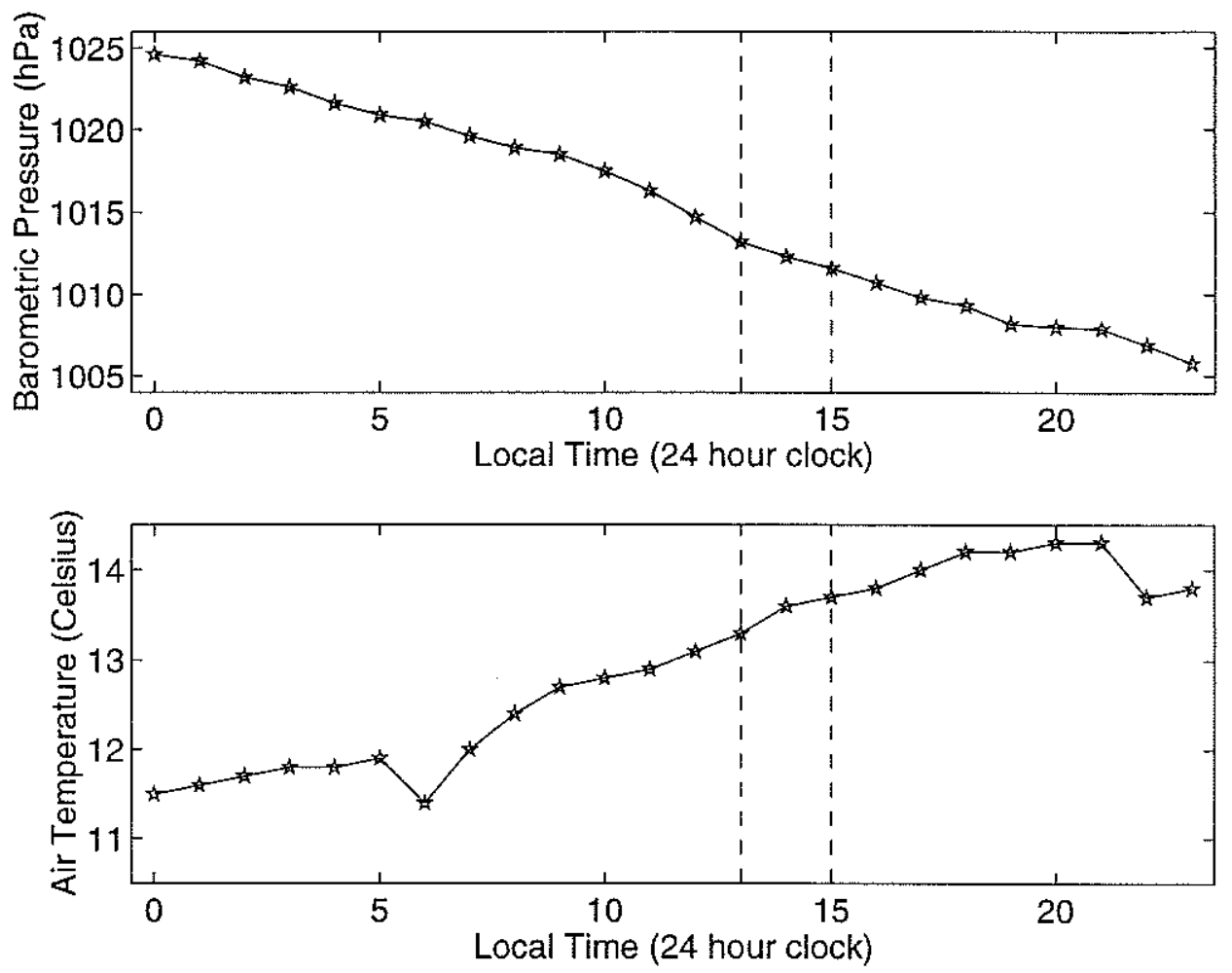

Figure 4-6: Hourly Barometric Pressure and Air Temperature from BUZM3 Tower. The barometer is located 17.4 meters above mean sea level and the temperature sensor is located 24.5 meters above mean sea level. The data points are marked with stars. The dashed lines indicate the approximate temporal duration of the experimental data collected with the author's instruments. 

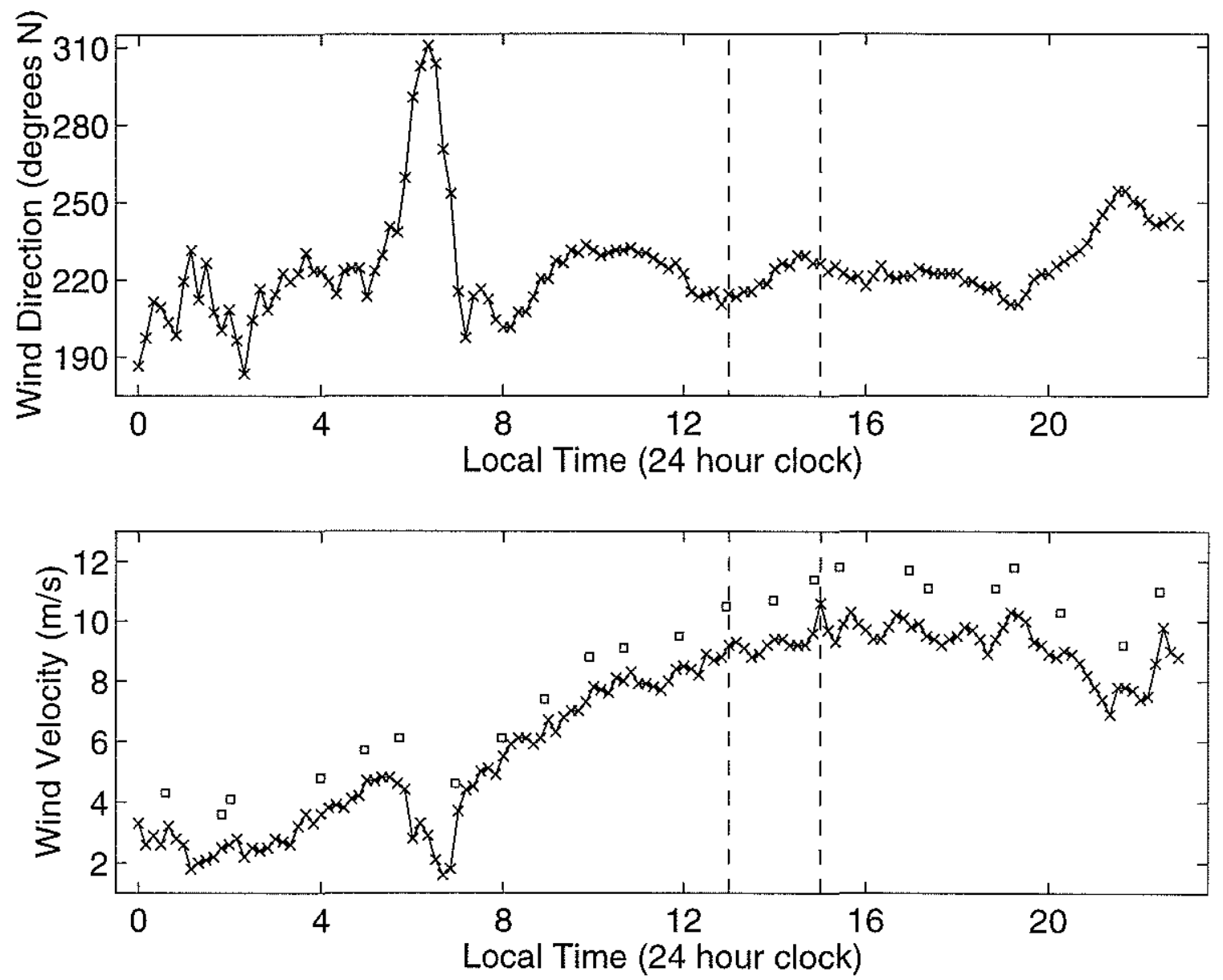

Figure 4-7: 10 Minute Averaged Wind Direction and Velocity from BUZM3 Anemometer. The anemometer is located 24.8 meters above mean sea level. The data points are marked with an ' $x$ '. The dashed lines indicate the approximate temporal duration of the experimental data collected with the author's instruments. The squares indicate the maximum wind velocity during each hour and when it occurred. 

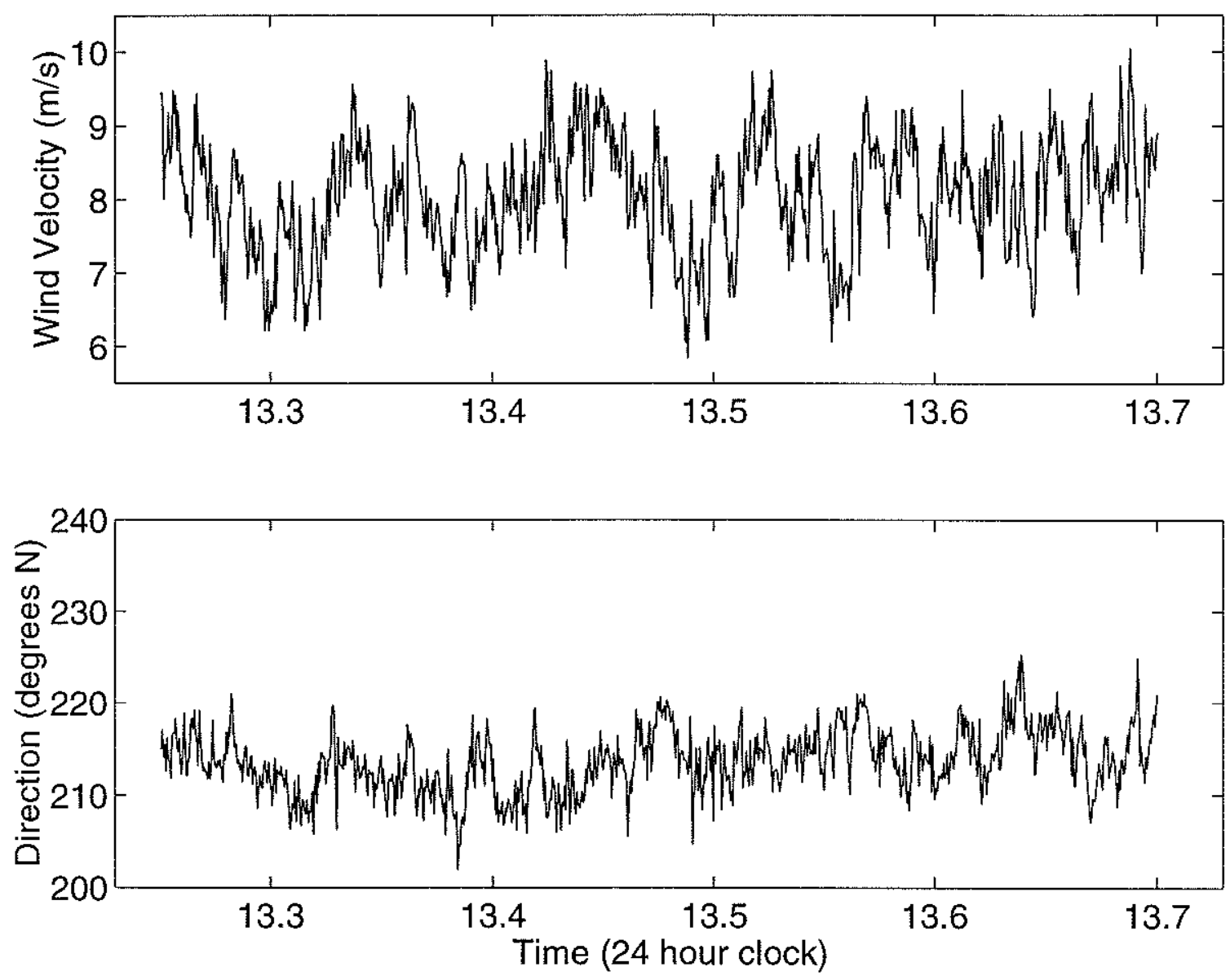

Figure 4-8: CSAT3 Sonic Anemometer Data from Data Set 10281315. The wind velocity is given at a 10 meter reference height. The data has been averaged over 2 second intervals. The mean wind direction is $213.7^{\circ}$ and the mean wind velocity is $8.05 \mathrm{~m} / \mathrm{s}$. 

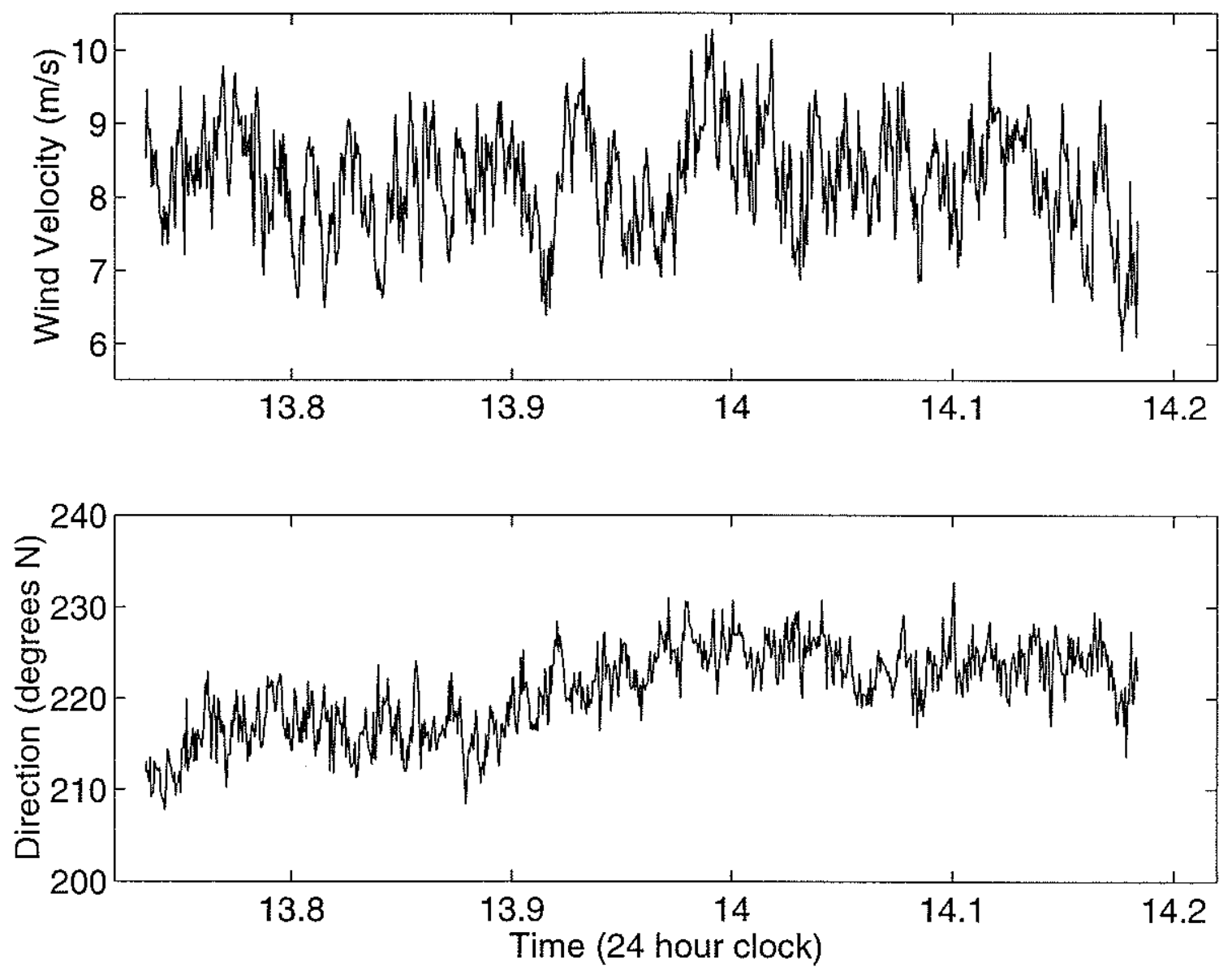

Figure 4-9: CSAT3 Sonic Anemometer Data from Data Set 10281344. The wind velocity is given at a 10 meter reference height. The data has been averaged over 2 second intervals. The mean wind direction is $220.9^{\circ}$ and the mean wind velocity is $8.23 \mathrm{~m} / \mathrm{s}$. 

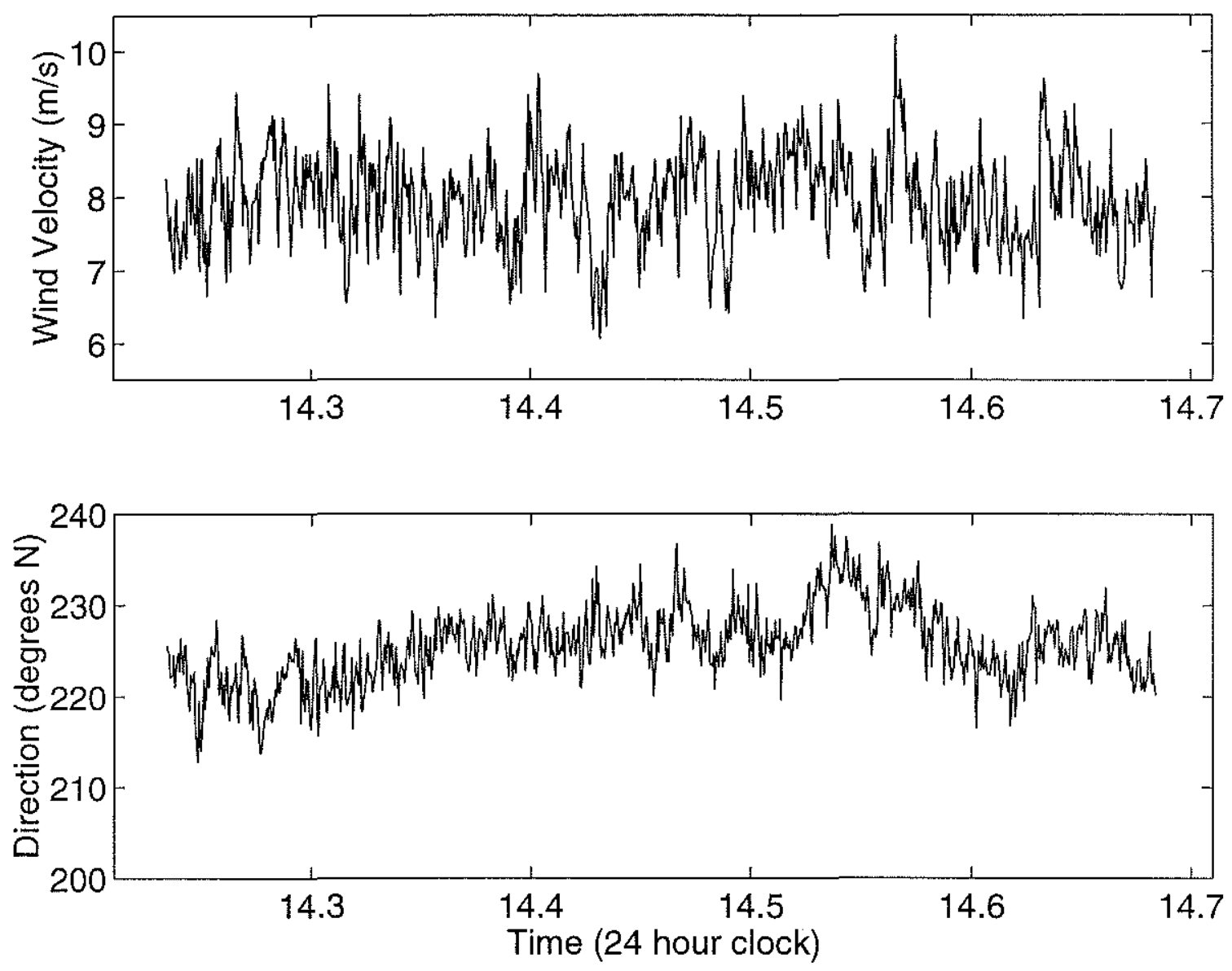

Figure 4-10: CSAT3 Sonic Anemometer Data from Data Set 10281414. The wind velocity is given at a 10 meter reference height. The data has been averaged over 2 second intervals. The mean wind direction is $225.6^{\circ}$ and the mean wind velocity is $7.96 \mathrm{~m} / \mathrm{s}$. 


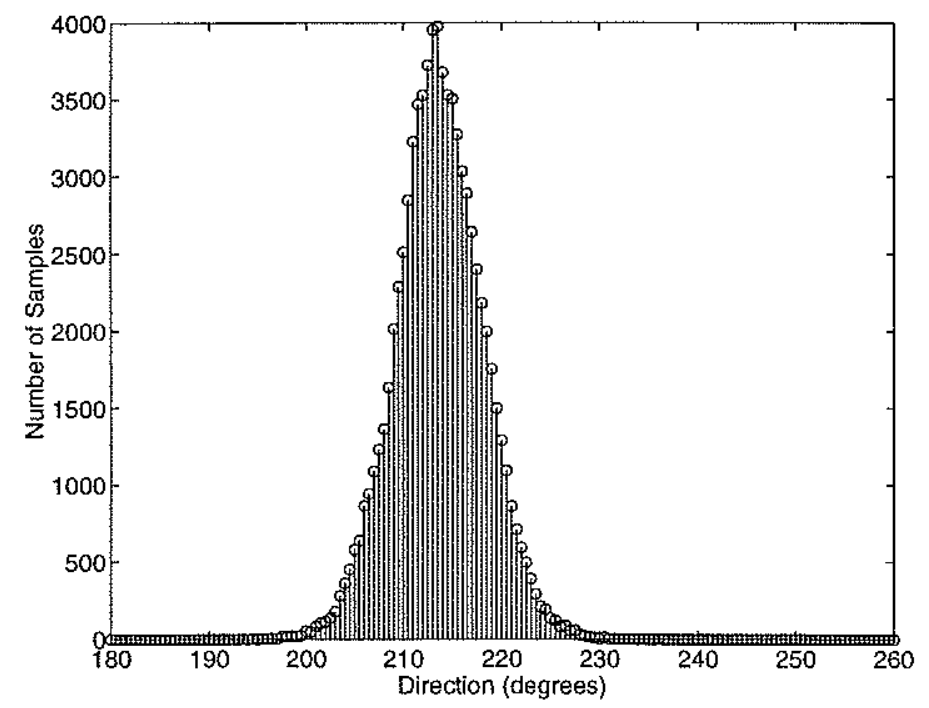

Figure 4-11: Wind Direction Histogram for Data Set 10281315.

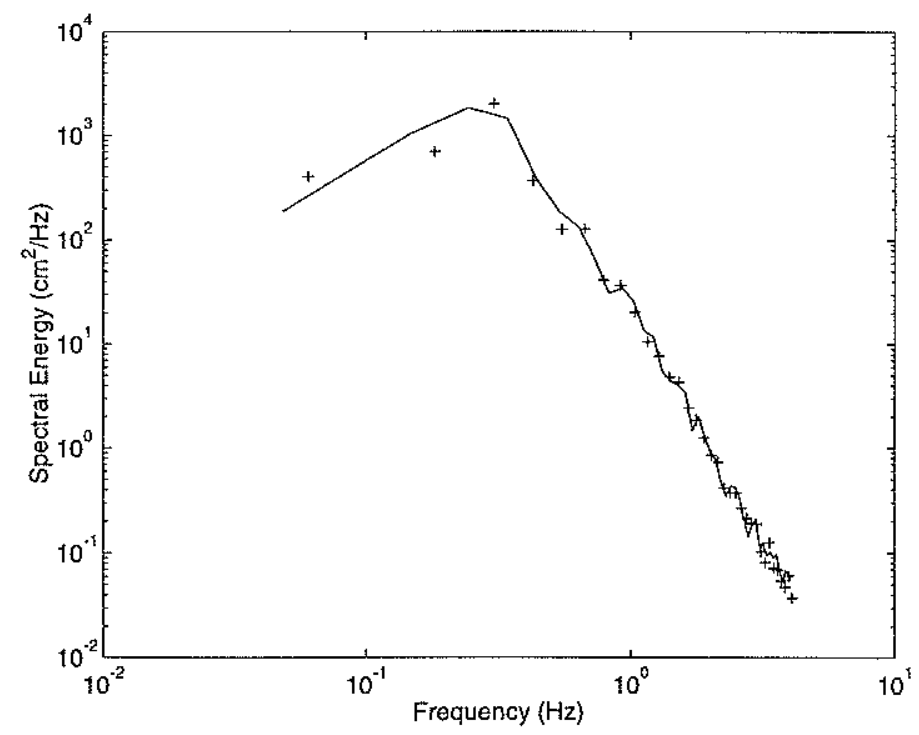

Figure 4-12: Capacitive Wire Wave Gage Single Wire Frequency Spectrum from Data Set 10281344. The pluses are the spectrum calculated from the first 8.7 minutes of the data set which was sampled at $1000 \mathrm{~Hz}$. The solid line represents a spectrum calculated from the first 10.9 minutes of the data set which was a subsampled version of the original with a sampling rate of $100 \mathrm{~Hz}$. The spectrum is shown only out to the resolution of the instrument. The noise floor for each channel plateaus at around $10 \mathrm{~Hz}$ between $10^{-2}$ and $10^{-3} \mathrm{~cm}^{2} / \mathrm{Hz}$. 


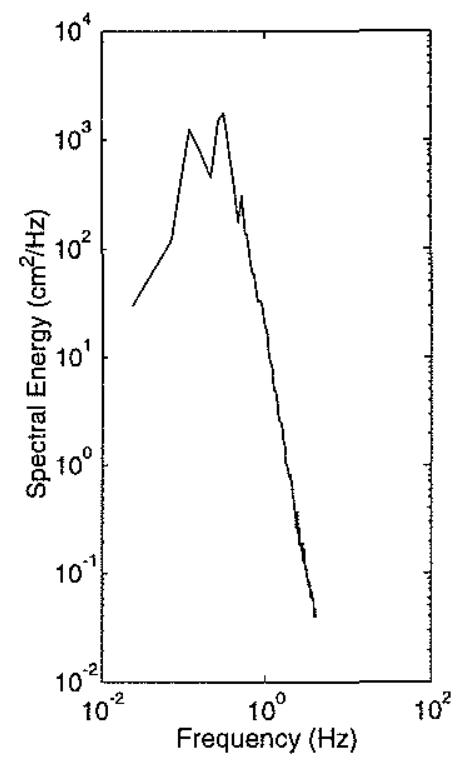

(a)

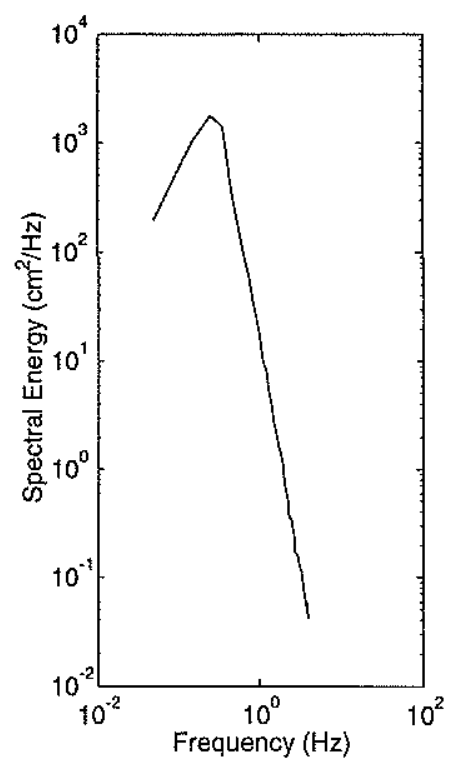

(b)

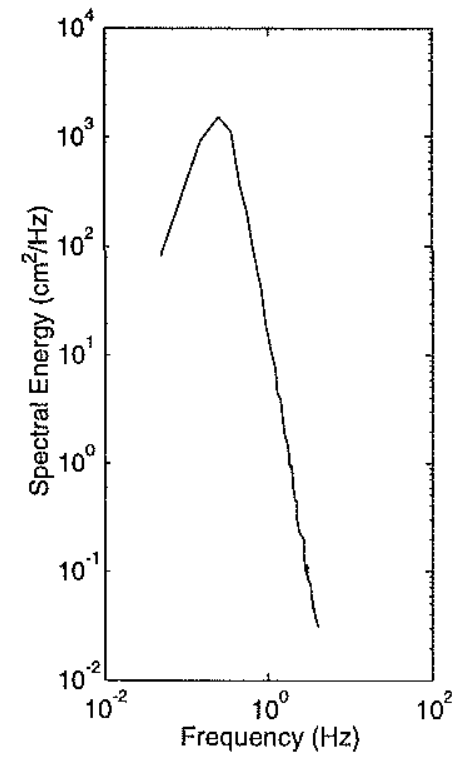

(c)

Figure 4-13: Capacitive Wire Wave Gage One-Dimensional Frequency Spectra Averaged From All 6 Wires From Data Sets 10281315, 10281344, and 10281414. Subfigure (a) was calculated from the first 21.8 minutes of data set 10281315, while subfigures (b) and (c) were calculated from the first 10.9 minutes of data sets 10281344 and 10281414 , respectively.

its shape indicating that while the atmospheric conditions were not stationary, the change in the long wave field energy density between the first and last data sets was not significant.

The directionality of the long wave spectrum is also available from the capacitive wire wave gage array. Plots of the two-dimensional frequency spectrum from each of the data files are given in Figures 4-14, 4-15, and 4-16. The direction from which the wind is blowing is indicated on each plot and correspond well with the calculated energy distribution which should appear 'downwind' on these plots due to the processing algorithm used (e.g. in Figure 4-14 the wind is coming from approximately $214^{\circ}$ and the energy is bunched near the 'downwind' direction of $\left.214-180=34^{\circ}\right)$. Their purpose, however, is to show general directional energy distribution, not specific details, as the resolution and method of deployment for the instrument were not designed for this. Additionally, the low frequency limit in the 
figures of $0.35 \mathrm{~Hz}$ is the result of the directional resolution limit warranted by the maximum wire spacing in the array: a $0.35 \mathrm{~Hz}$ wave has a wavelength of approximately 12.6 meters. The high frequency directional limit of the instrument is $2.29 \mathrm{~Hz}$ corresponding to a wave having a 0.3 meter wavelength. The plots do not extend out to this higher limit because the energy density is plotted on a linear scale, and the power law fall-off of energy density would prevent the representation of energy levels at these higher frequencies. The peaks in the directional energy distribution plots between 0.4 and $0.5 \mathrm{~Hz}$ are secondary peaks in the spectrum which can be seen in the higher resolution point spectrum subfigure (a) in Figure 4-13.

These spectra are computed using a two-dimensional form of the Data Adaptive Spectral Estimator (DASE) proposed by Davis and Regier [15]. This method attempts to estimate a continuous two-dimensional spectrum, $\Phi(k)$, where $k$ is a vector, using the following approximation

$$
<\hat{\Phi}(\boldsymbol{k})>=\int d \boldsymbol{k}^{\prime} \Phi\left(\boldsymbol{k}^{\prime}\right) W\left(\boldsymbol{k}, \boldsymbol{k}^{\prime}\right) W\left(\boldsymbol{k}, \boldsymbol{k}^{\prime}\right)=\sum_{n, m} \alpha_{n m}(\boldsymbol{k}) \exp \left(i \boldsymbol{k}^{\prime} \cdot\left[\boldsymbol{x}_{n}-\boldsymbol{x}_{m}\right]\right)
$$

where $W\left(k, k^{\prime}\right)$ is the

wavenumber window and measures how much an estimate of the spectrum at wavenumber $k$ is affected by the variance density at wavenumber $k^{\prime}$ in exactly the way that the sinc function frequency window encountered in conventional time series analysis measures variance spreading in frequency space. Data adaptive spectral estimators do not try to approximate $W$ with a delta function, however, as in conventional a priori analysis methods. Instead the DASE method attempts to make $W\left(k, k^{\prime}\right)$ small at those values of $k^{\prime} \neq k$ where $\Phi(k)$ is significant.

- Davis and Regier [15]

Another noteworthy detail about this analysis method is that it constrains the wave energy in the estimated spectrum to lie only along the dispersion curve. While this limitation is an approximation, as discussed in Section 2.1 , for gravity waves it is a reasonable one and 


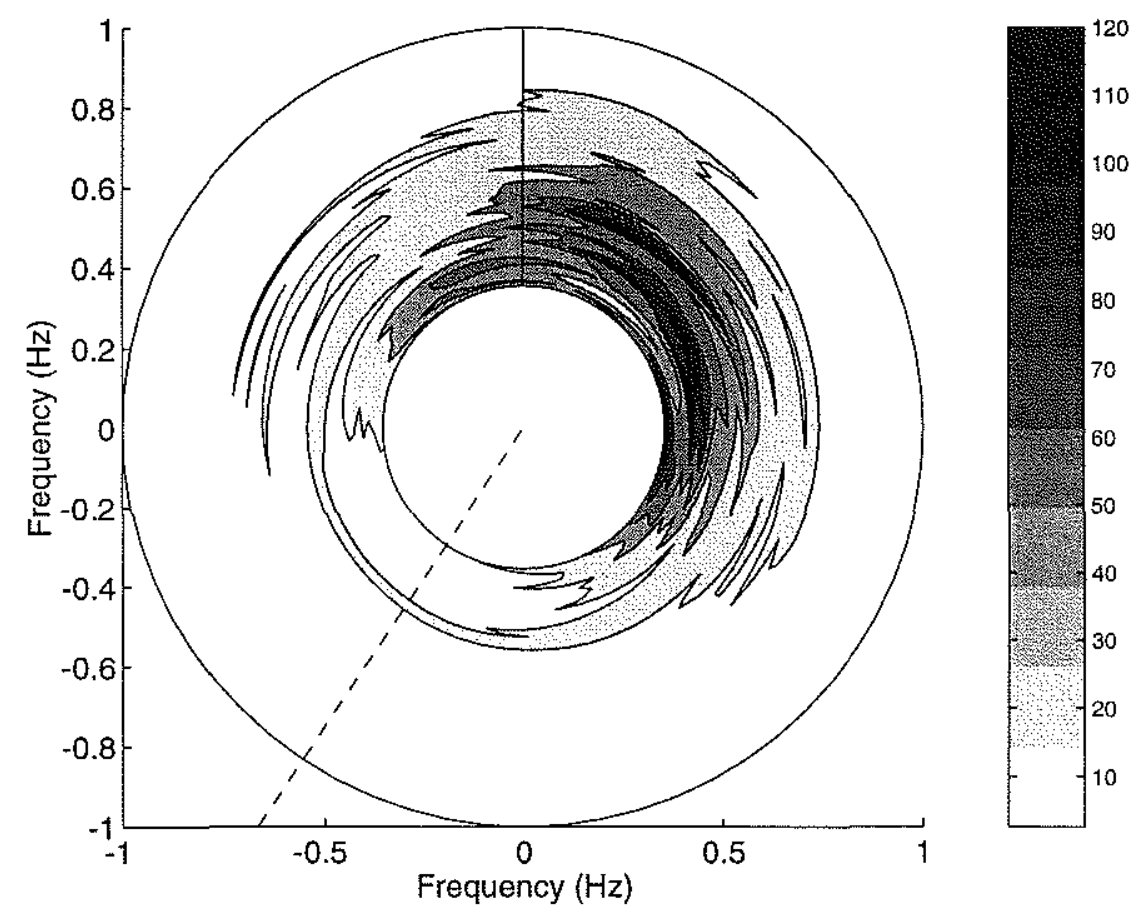

Figure 4-14: Capacitive Wire Wave Gage Two-Dimensional Frequency Spectrum from Data Set 10281315. The spectral estimate has units of $\mathrm{cm}^{2} /\left(\mathrm{Hz}^{2}\right)$ and gets darker as the energy level increases. North is pointing toward the top of the page and East is pointing to the right. The dashed line from the edge into the center of the plot shows the mean wind direction during the data set. Energy is only shown between the bands of $0.35 \mathrm{~Hz}$ and 1 $\mathrm{Hz}$. This estimate is based on the first 21.8 minutes of the data set. 


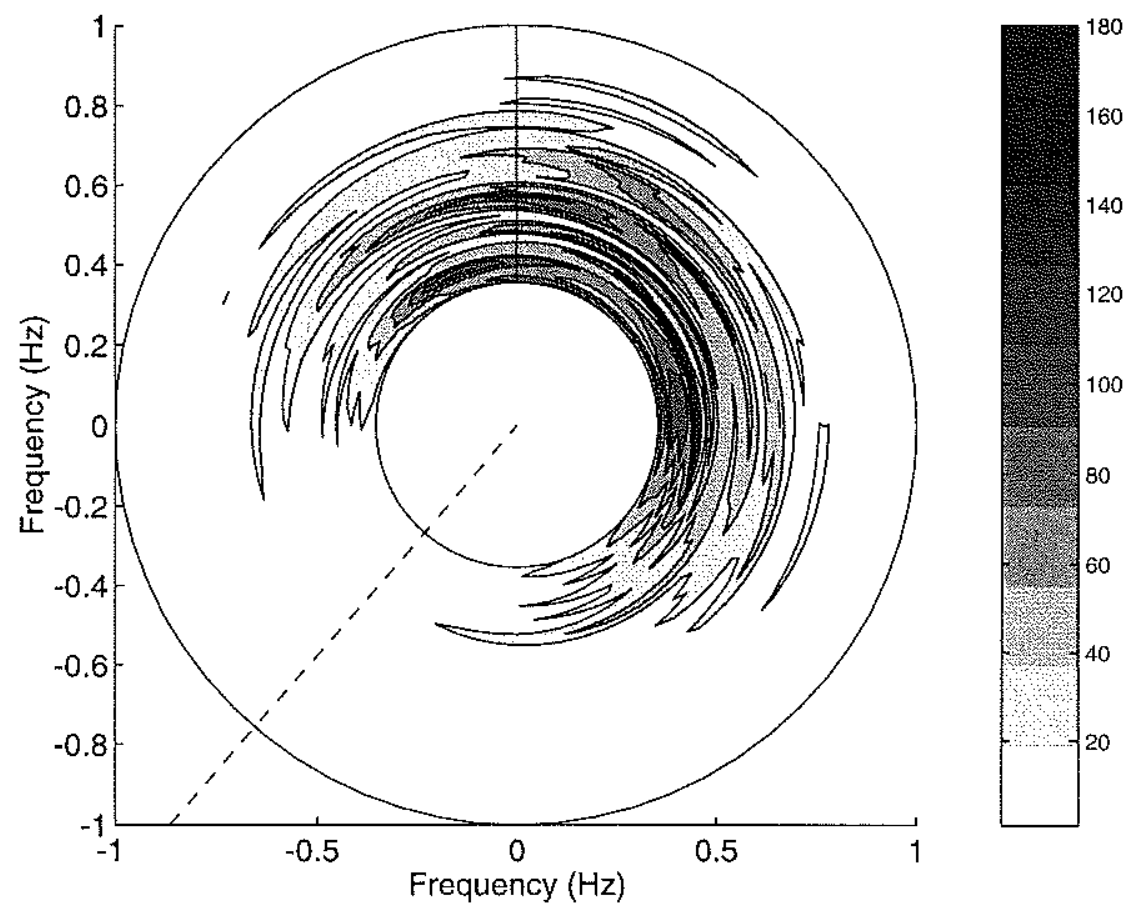

Figure 4-15: Capacitive Wire Wave Gage Two-Dimensional Frequency Spectrum from Data Set 10281344. The spectral estimate has units of $\mathrm{cm}^{2} /\left(\mathrm{Hz}^{2}\right)$ and gets darker as the energy level increases. North is pointing toward the top of the page and East is pointing to the right. The dashed line from the edge into the center of the plot shows the mean wind direction during the data set. Energy is only shown between the bands of $0.35 \mathrm{~Hz}$ and 1 $\mathrm{Hz}$. This estimate is based on the first 10.9 minutes of the data set. 

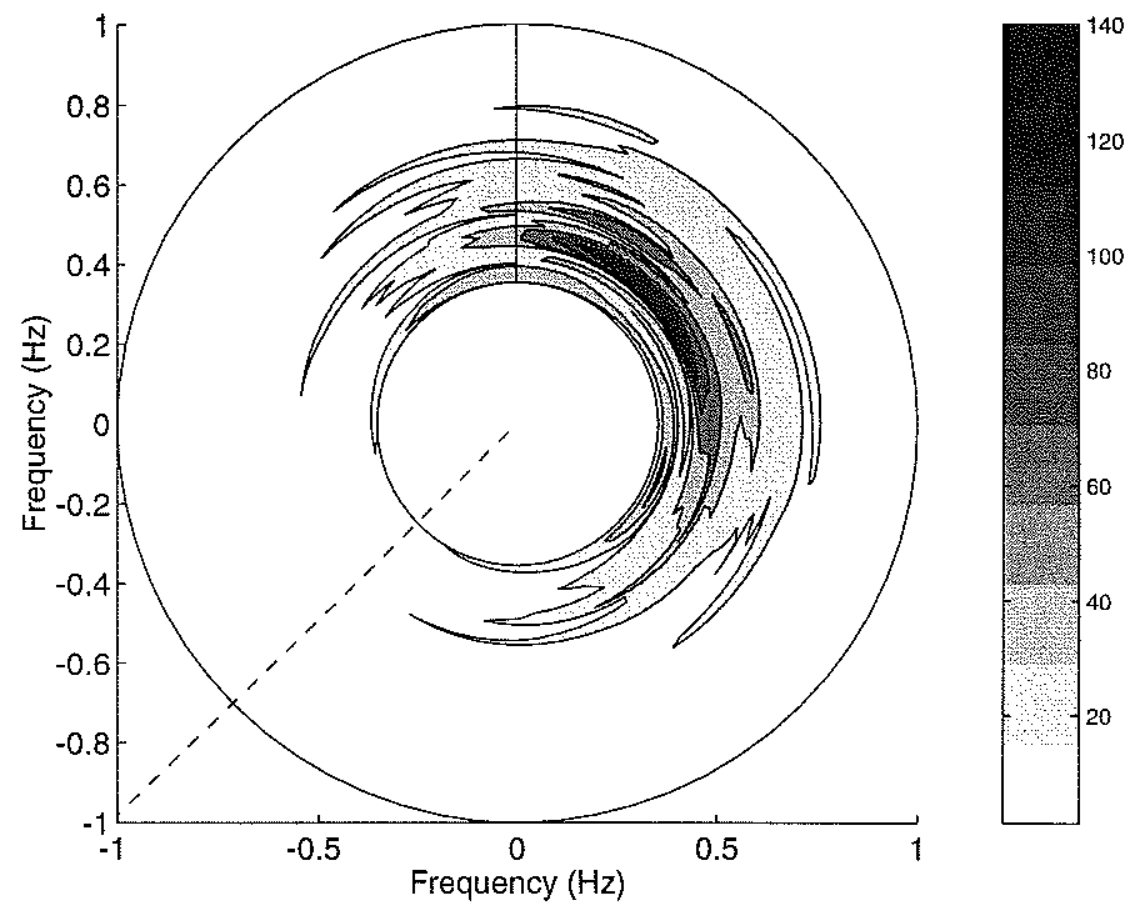

Figure 4-16: Capacitive Wire Wave Gage Two-Dimensional Frequency Spectrum from Data Set 10281414. The spectral estimate has units of $\mathrm{cm}^{2} /\left(\mathrm{Hz}^{2}\right)$ and gets darker as the energy level increases. North is pointing toward the top of the page and East is pointing to the right. The dashed line from the edge into the center of the plot shows the mean wind direction during the data set. Energy is only shown between the bands of $0.35 \mathrm{~Hz}$ and 1 $\mathrm{Hz}$. This estimate is based on the first 10.9 minutes of the data set. 
is widely used for long-wave spectral estimation formulations.

\section{Scatterometer Measurements}

The scatterometer data was processed as follows. The raw output files constitute a time series of voltages collected as the instrument rotates $180^{\circ}+$ clockwise and then $180^{\circ}+$ counterclockwise. First the raw data was separated into periods corresponding to specific sectors of rotation. The angular rotation was measured using a potentiometer mounted on the motor axis. The power for each polarization was calculated for each sector by squaring the in-phase and quadrature measured signals and then adding them together. Each consecutive arc traveled the opposite direction from the previous one, so the power from alternate data periods was averaged. Then each was shifted so the same geographic directional sectors were aligned and then they were averaged together. The resulting directional power averages, one for $\mathrm{HH}$ polarization and one for $\mathrm{VV}$, were referenced to magnetic North by comparing the known rotation rate and total rotation angle of the scatterometer with its position when mounted on the tower. The uncertainty in absolute direction was about $5^{\circ}$. It should be noted that the time between each illumination by the scatterometer of a specific location on the water surface was not equal. This was due to the reversal of direction of the scatterometer scan. Even though the ocean surface is not truly ergodic either on the day of the experiment or in general, the uneven spacing of the data samples which are averaged is assumed not to affect the resulting directional power plots.

Figures 4-17, 4-18, and 4-19 show the averaged backscattered power measured by the scatterometer for both polarizations during each of the data sets. The total averaging time for each data set is approximately 26 minutes. The data represents only the $180^{\circ}$ of rotation when the scatterometer was viewing open water (the extra $15^{\circ}$ at each end of the rotation has been removed for clarity). The data was not entirely circular, meaning there may have been small variations in the phase shift of the output signals, so corrections were made including a general correction to equilibrate overall gain between the I and Q channels for each polarization. The description of these modifications can be found in an internal report by J. R. Jensen [35] at the Johns Hopkins University Applied Physics Laboratory. 


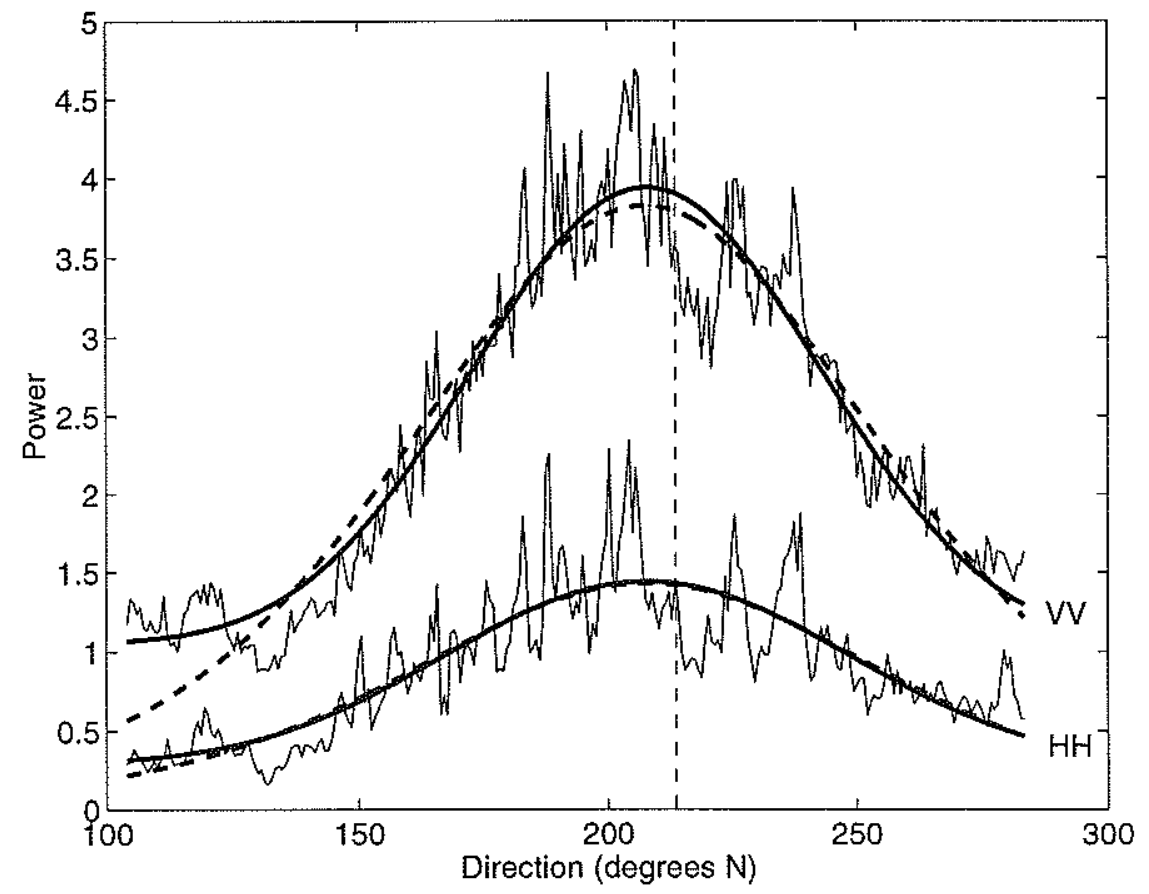

Figure 4-17: Directional X-band Scatterometer Return from Data Set 10281315. The power is calculated using $\mathrm{I}^{2}+\mathrm{Q}^{2}$ where $\mathrm{I}$ and $\mathrm{Q}$ are measured in volts. The thick solid line represents a best fit of the data to the function $\cos ^{2 s}\left(\frac{\theta-\theta_{\max }}{2}\right)$, while the thick dashed line represents a best fit of the data to the function $\cosh ^{-2}\left(\beta\left(\theta-\theta_{\max }\right)\right)$. The $s$ and $\beta$ values for the $\mathrm{HH}$ and VV polarizations are $4.02,0.879$ and $5.31,0.890$, respectively. The data points shown are 85 point averages. The thin vertical dashed line is the mean wind direction during the data set of $\left(214^{\circ}\right)$ 


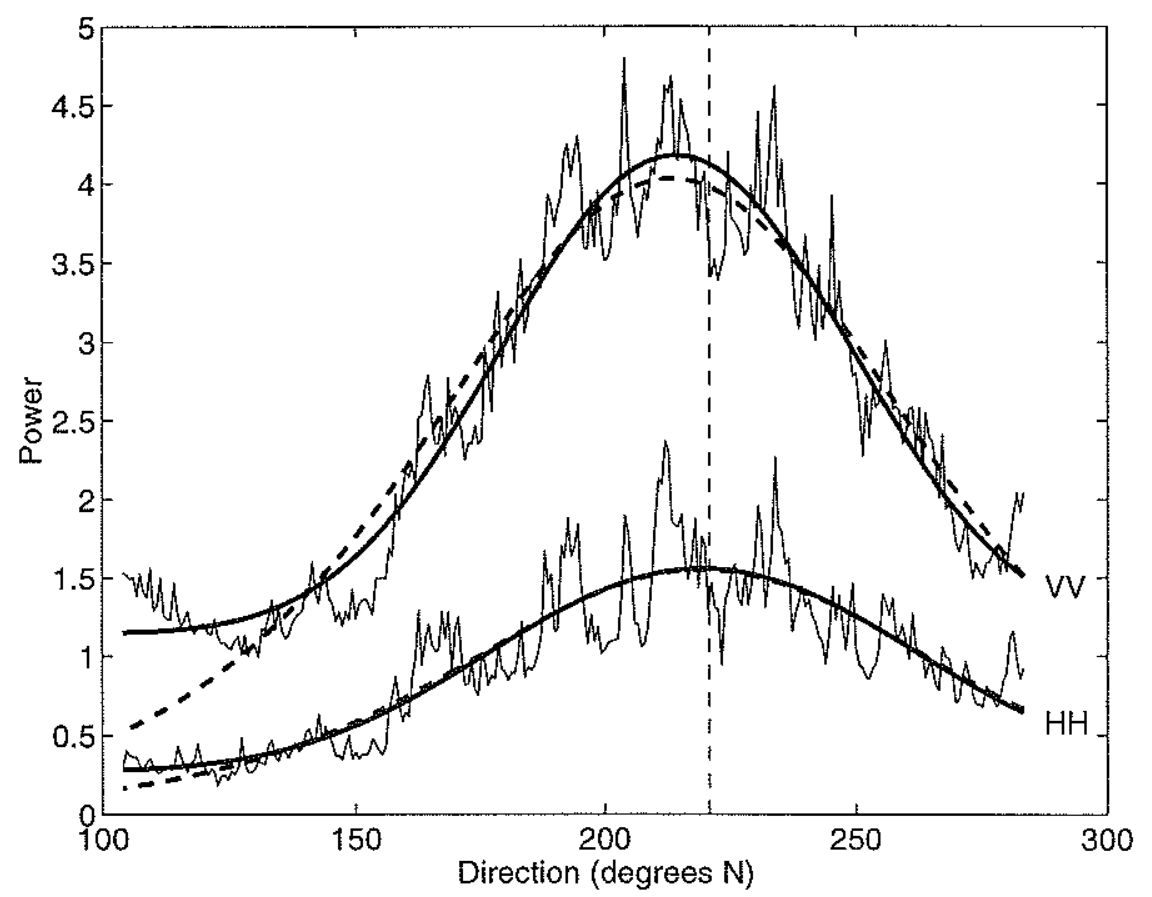

Figure 4-18: Directional X-band Scatterometer Return from Data Set 10281344. The power is calculated using $\mathrm{I}^{2}+\mathrm{Q}^{2}$ where $\mathrm{I}$ and $\mathrm{Q}$ are measured in volts. The thick solid line represents a best fit of the data to the function $\cos ^{2 s}\left(\frac{\theta-\theta_{\max }}{2}\right)$, while the thick dashed line represents a best fit of the data to the function $\cosh ^{-2}\left(\beta\left(\theta-\theta_{\max }\right)\right)$. The $s$ and $\beta$ values for the $\mathrm{HH}$ and VV polarizations are $3.79,0.883$ and $5.44,0.878$, respectively. The data points shown are 85 point averages. The thin vertical dashed line is the mean wind direction during the data set of $\left(221^{\circ}\right)$. 


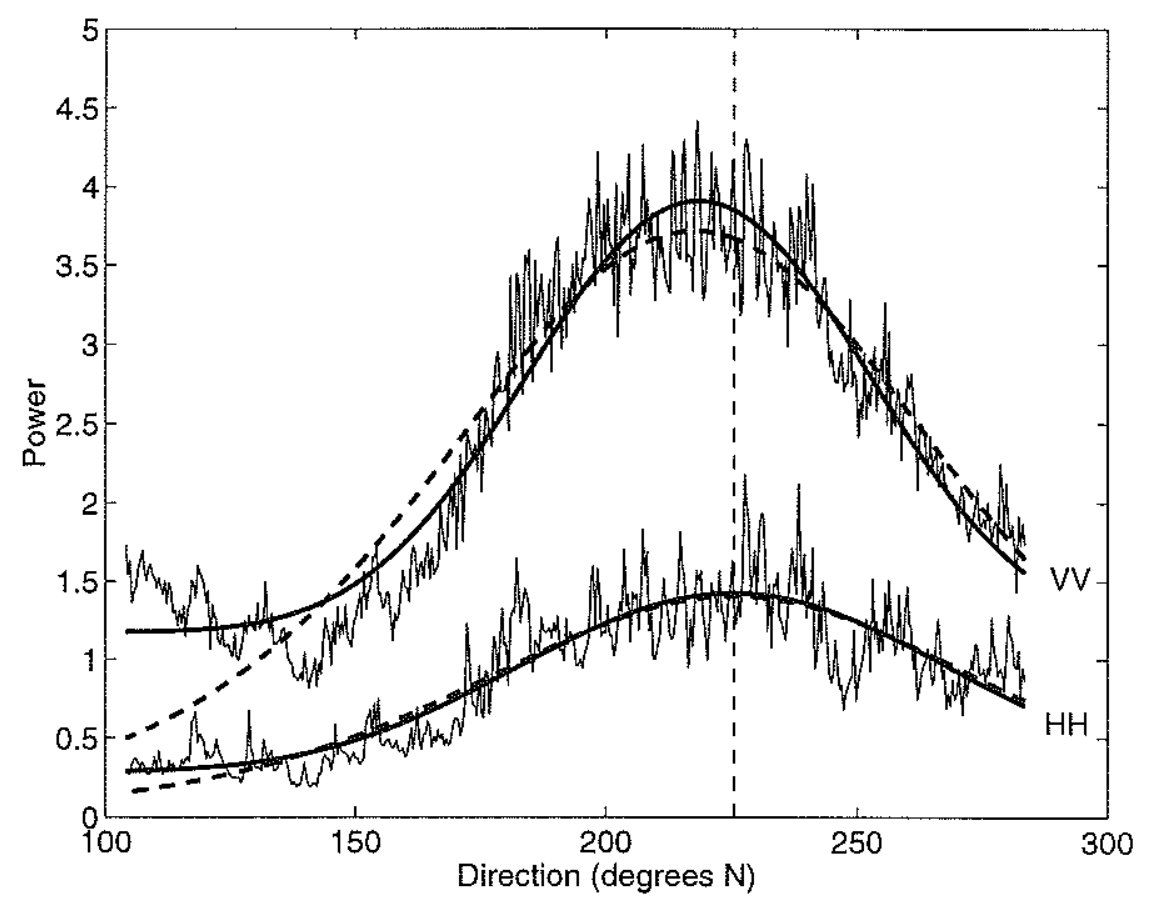

Figure 4-19: Directional X-band Scatterometer Return from Data Set 10281414. The power is calculated using $\mathrm{I}^{2}+\mathrm{Q}^{2}$ where $\mathrm{I}$ and $\mathrm{Q}$ are measured in volts. The thick solid line represents a best fit of the data to the function $\cos ^{2 s}\left(\frac{\theta-\theta_{\max }}{2}\right)$, while the thick dashed line represents a best fit of the data to the function $\cosh ^{-2}\left(\beta\left(\theta-\theta_{\max }\right)\right)$. The $s$ and $\beta$ values for the $\mathrm{HH}$ and $\mathrm{VV}$ polarizations are $3.72,0.831$ and $5.73,0.839$, respectively. The data points shown are 53 point averages. The thin vertical dashed line is the mean wind direction during the data set of $\left(226^{\circ}\right)$. 


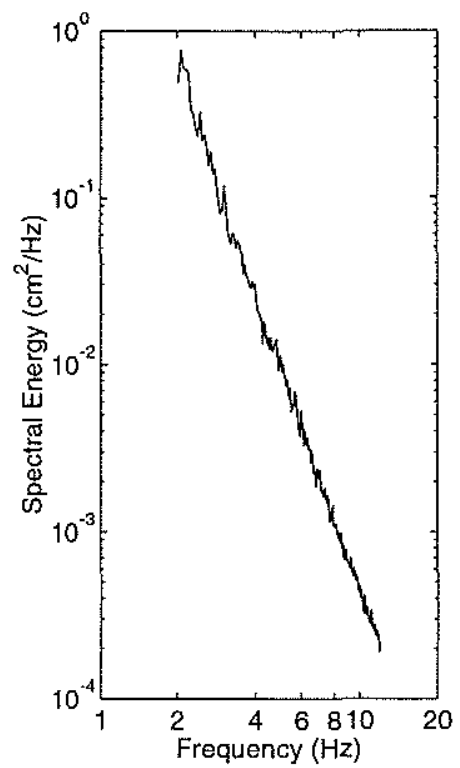

(a)

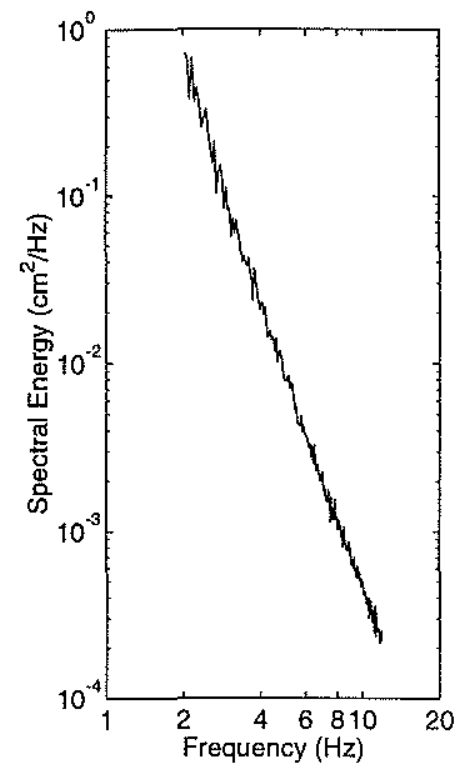

(b)

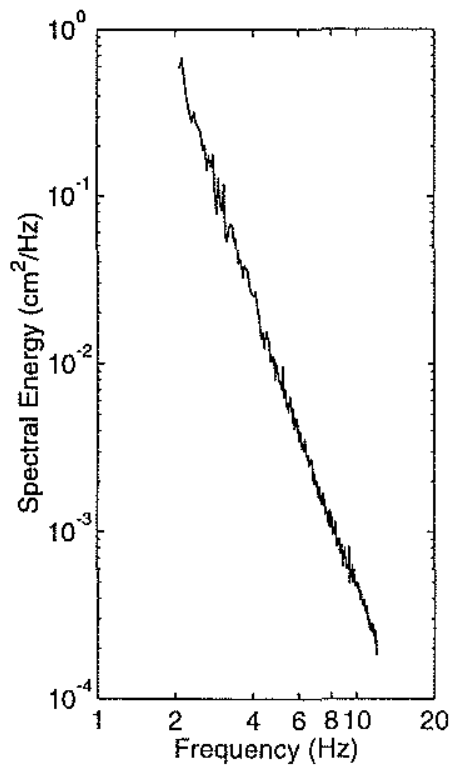

(c)

Figure 4-20: Circular Wire Wave Gage Frequency Spectrum Averaged from 16 Single Wire Spectra from Data Sets 10281415, 10281445, and 10281515. Subfigure (a) is from data set $10281415,(b)$ is from data set 10281445 , and (c) is from data set 10281515.

\section{Circular Wire Wave Gage Measurements}

The frequency spectra from approximately 21 minutes of calibrated time series data from the three different circular wire wave gage data sets are shown in Figure 4-20. Each spectrum is an average of the frequency spectra from the first sixteen channels of the instrument. All the data set file names are based on the month, day, hour, and minute of collection corresponding to Eastern Daylight Time (EDT), however, the circular wire wave gage used Eastern Standard Time (EST, where EST-1 = EDT), so while the data sets were collected at 1315,1345 , and $1415 \mathrm{EDT}$, their file names are 10281415, 10281445, and 10281515, respectively.

Two-dimensional wave height spectra are presented in Figures 4-21, 4-22, and 4-23, where each spectrum was calculated from data averaged over approximately 21 minutes. It should be noted that the compass directions on these plots are not standard: North is 
upwards on the page, but West is to the right. Additionally, the spectrum is symmetric through the origin because of its definition as being the Fourier transform of an autocorrelation which is, itself, symmetric through the origin. Because of this symmetry only half of the spectrum is shown in the two-dimensional spectrum plots.

A one-dimensional wavenumber spectrum can be generated from each of the two-dimensional spectral plots by integrating out the angular dimensions, such that given a symmetric twodimensional spectrum of the form $\Phi(k, \theta)$ with $\theta$ only given over a $180^{\circ}$ range, (Figures 4-21 through 4-23 can be converted to this form), then an omnidirectional spectrum can be generated using

$$
S(k)=2 \int_{0}^{\pi} \Phi(k, \theta) k d \theta
$$

The omnidirectional spectra calculated in this manner from the circular wire wave gage two-dimensional wavenumber spectra are plotted in Figure 4-24.

A more detailed look at the directional spectrum plots can be obtained by taking circular slices of the spectrum. Figure 4-25 shows some of these slices, each depicting the actual directional distribution of the energy at a given wavelength. The white areas in the twodimensional wavenumber spectral plots and the points along the directional energy slices which are equal to zero represent areas where the energy estimate is either zero or negative. The processing algorithms allow negative energy due to small negative side lobes caused by filtering, but negative energy is not physically consistent, so any negative energy areas were set to zero. In general, the magnitude of the negative energy lies within the magnitude of the uncertainty of the spectral estimate.

\subsection{Comparison of Data Processing Methods}

In order to verify that the analysis method being used by the circular wire wave gage does indeed reproduce the appropriate estimate of the spectrum, it was compared to the data processing used by a scanning laser slope gage (SLSG) which also has the ability to measure the two-dimensional wavenumber frequency. If an SLSG calibrated slope time series data is used to estimate the slope spectrum using the circular wire wave gage processing, and 


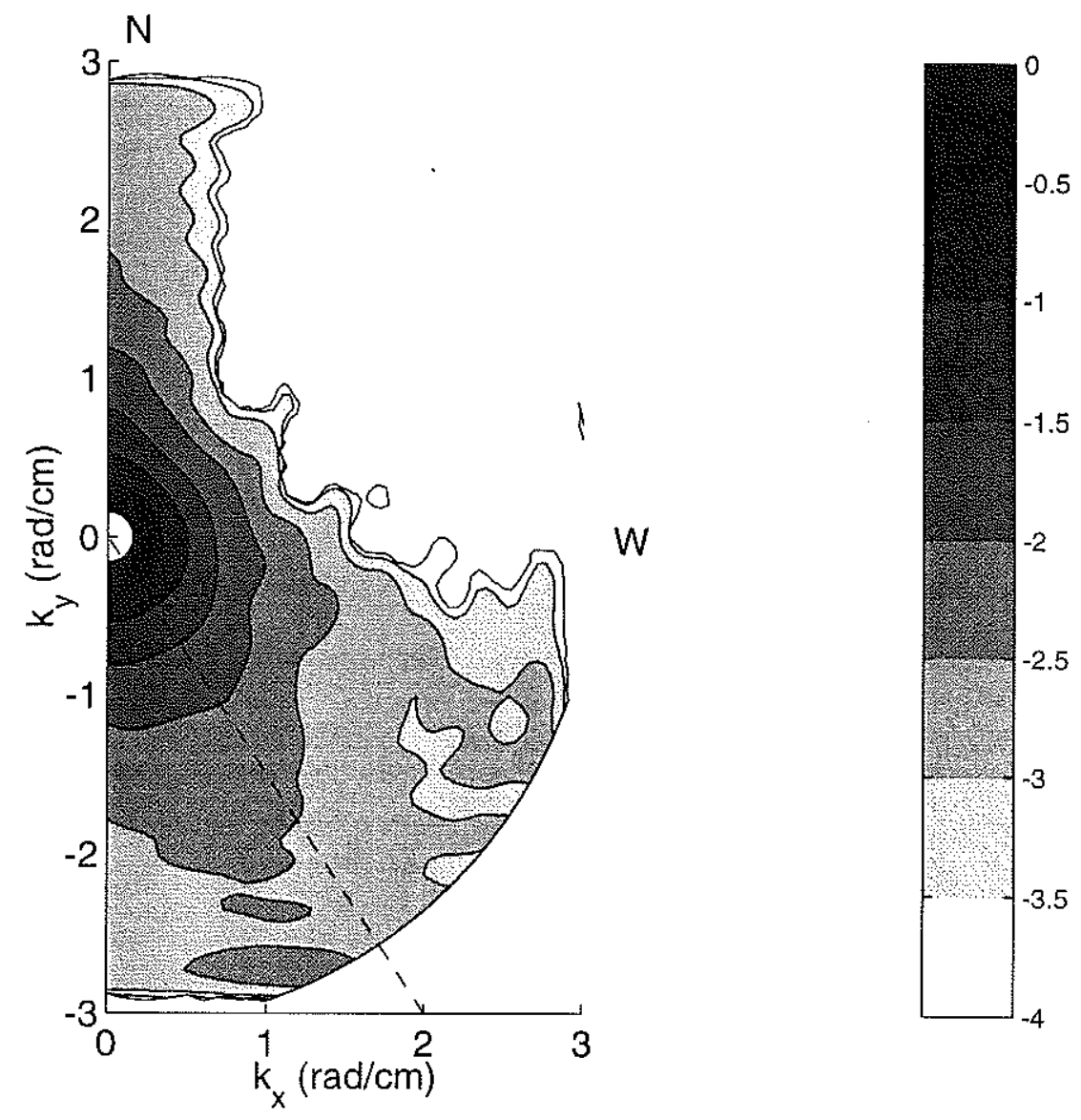

Figure 4-21: Circular Wire Wave Gage Two-dimensional Wave Height Spectrum from Data Set 10281415 (corresponding to 1315 on October 28, 1998). The spectral energy, $\Phi\left(k_{x}, k_{y}\right)$, has units of $\mathrm{cm}^{2} /(\mathrm{rad} / \mathrm{cm})^{2}$ and the colorbar scale reflects the spectral energy plotted as $\log _{10}\left(\Phi\left(k_{x}, k_{y}\right)\right)$. The darker the color, the higher the energy level. North is pointing toward the top of the page and West is pointing to the right. The dashed line is the mean wind direction as measured at the BUZM3 tower over the approximate time period that this data set was collected. This estimate is based on the first 21 minutes of the data set. 


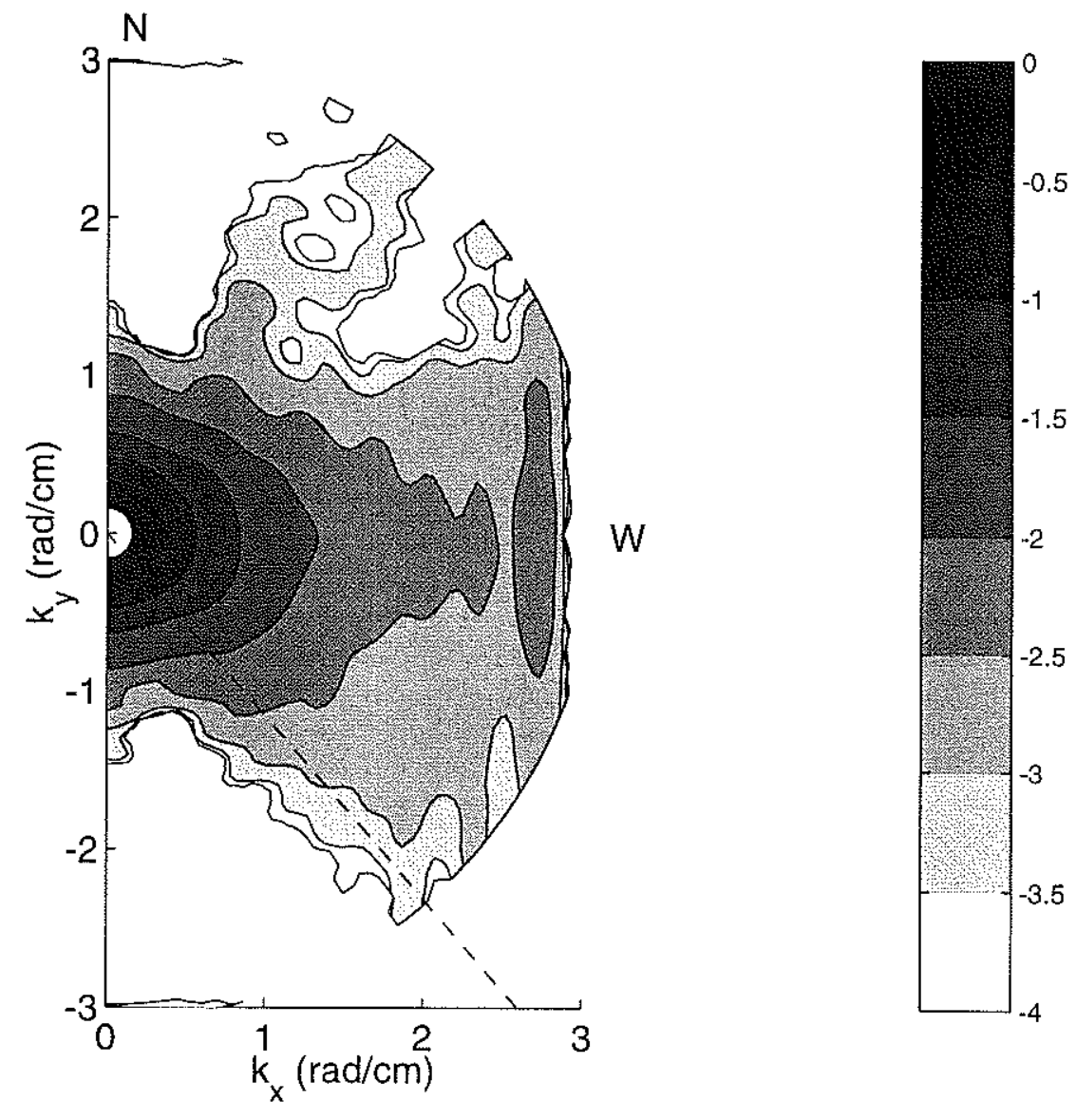

Figure 4-22: Circular Wire Wave Gage Two-dimensional Wave Height Spectrum from Data Set 10281445 (corresponding to 1345 on October 28, 1998). The spectral energy, $\Phi\left(k_{x}, k_{y}\right)$, has units of $\mathrm{cm}^{2} /(\mathrm{rad} / \mathrm{cm})^{2}$ and the colorbar scale reflects the spectral energy plotted as $\log _{10}\left(\Phi\left(k_{x}, k_{y}\right)\right)$. The darker the color, the higher the energy level. North is pointing toward the top of the page and West is pointing to the right. The dashed line is the mean wind direction as measured at the BUZM3 tower over the approximate time period that this data set was collected. This estimate is based on the first 21 minutes of the data set. 


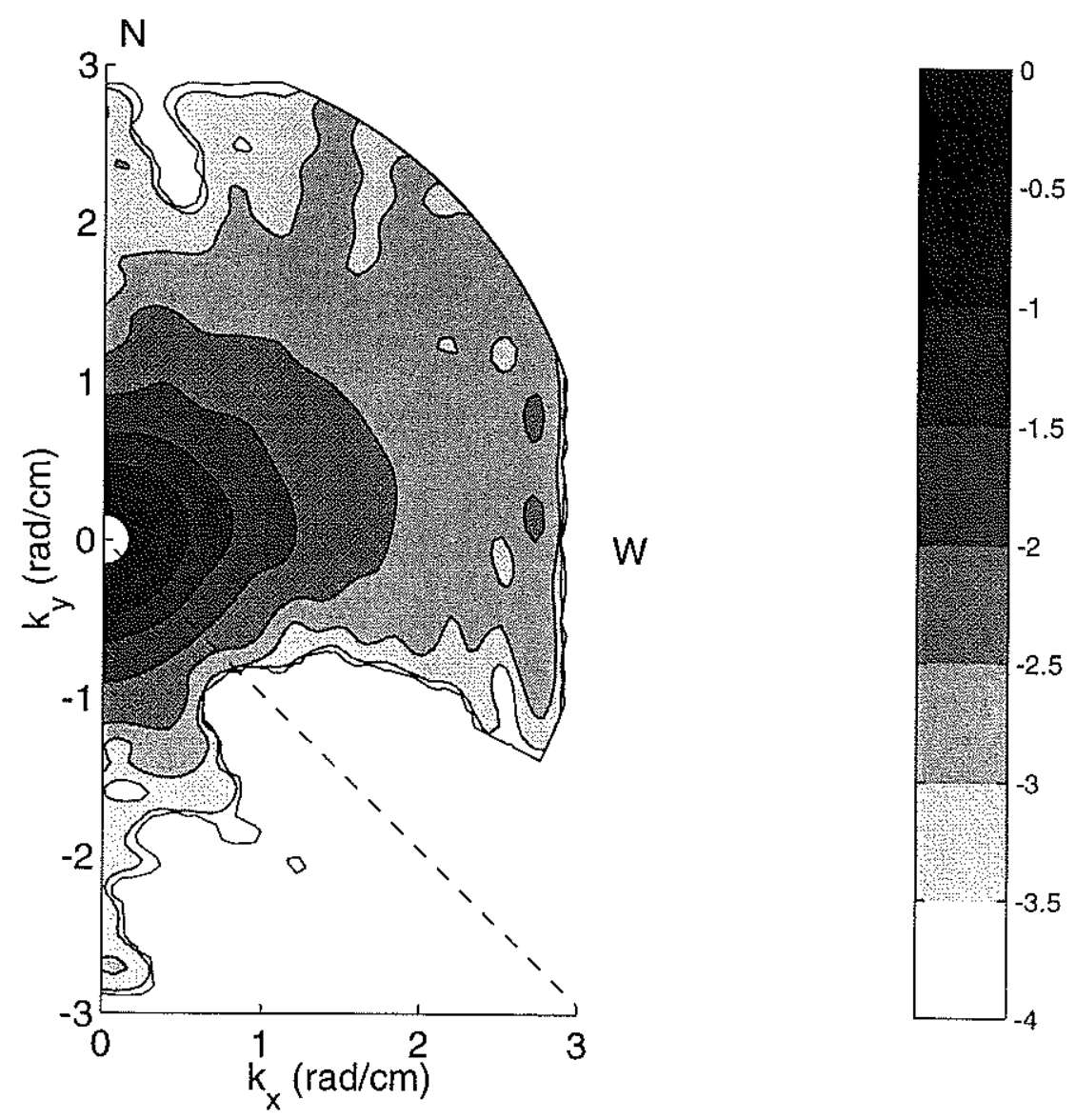

Figure 4-23: Circular Wire Wave Gage Two-dimensional Wave Height Spectrum from Data Set 10281515 (corresponding to 1415 on October 28, 1998). The spectral energy, $\Phi\left(k_{x}, k_{y}\right)$, has units of $\mathrm{cm}^{2} /(\mathrm{rad} / \mathrm{cm})^{2}$ and the colorbar scale reflects the spectral energy plotted as $\log _{10}\left(\Phi\left(k_{x}, k_{y}\right)\right)$. The darker the color, the higher the energy level. North is pointing toward the top of the page and West is pointing to the right. The dashed line is the mean wind direction as measured at the BUZM3 tower over the approximate time period that this data set was collected. This estimate is based on the first 21 minutes of the data set. 


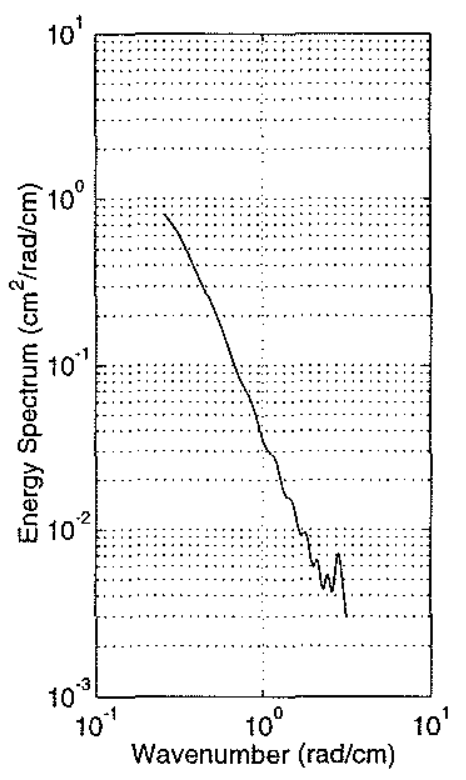

(a)

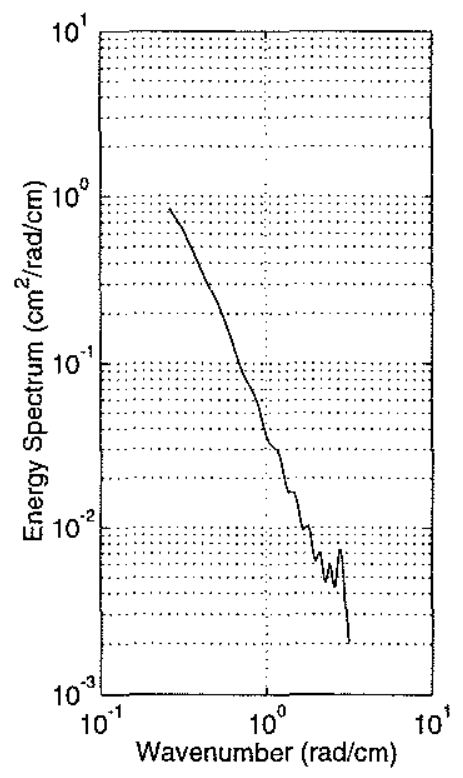

(b)

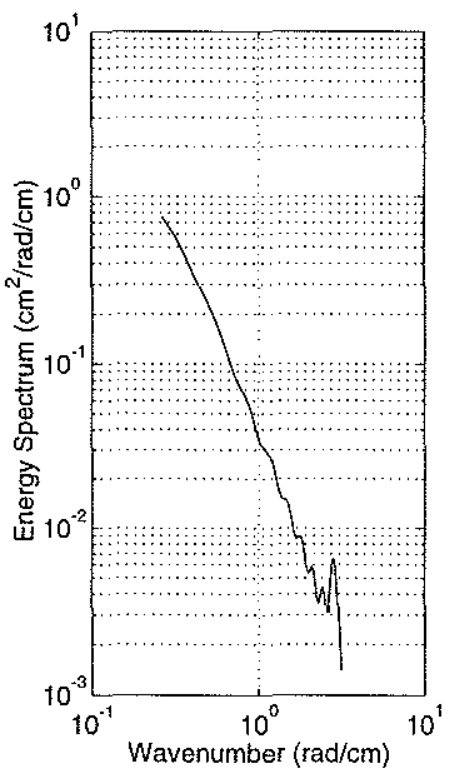

(c)

Figure 4-24: Omnidirectional Spectra from the Circular Wire Wave Gage Data Sets 10281415, 10281445, and 10281515. Each spectrum was generated using 21 minutes of data. Subfigure (a) is from data set 10281415, (b) is from data set 10281445, and (c) is from data set 10281515 . 


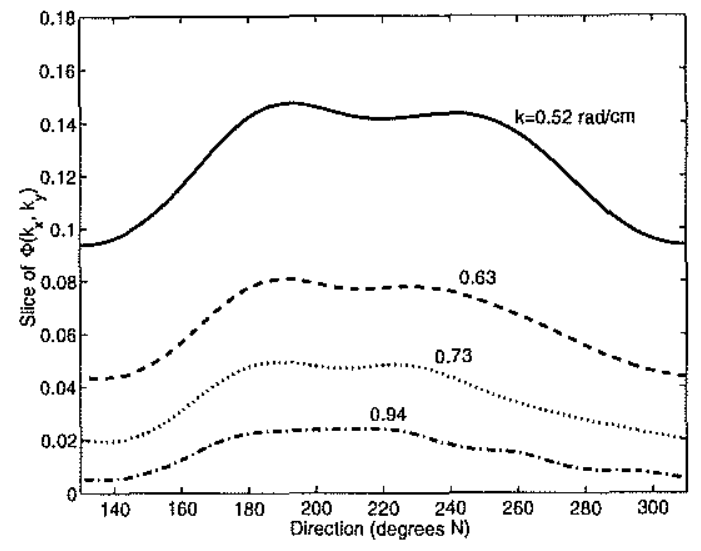

(a)

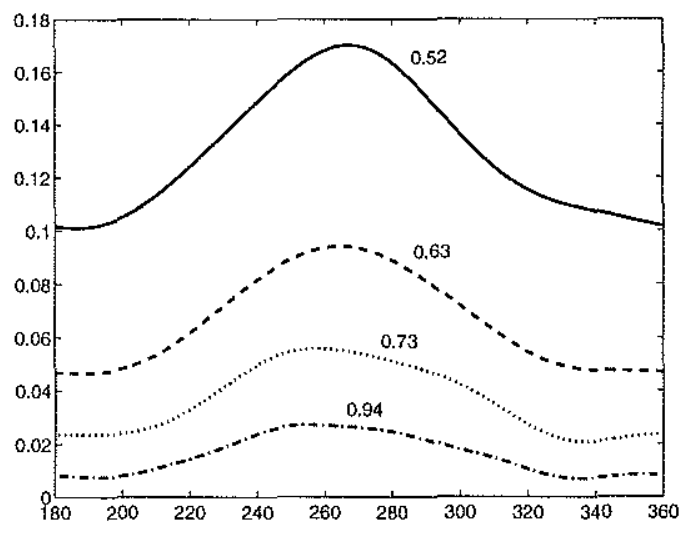

(c)

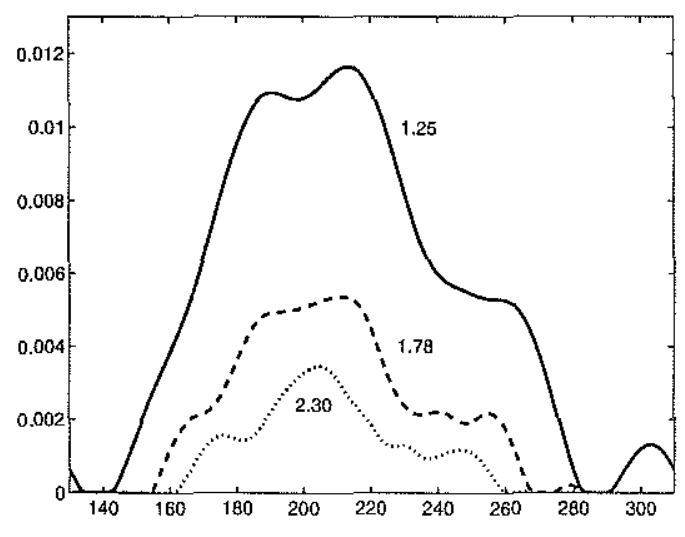

(b)

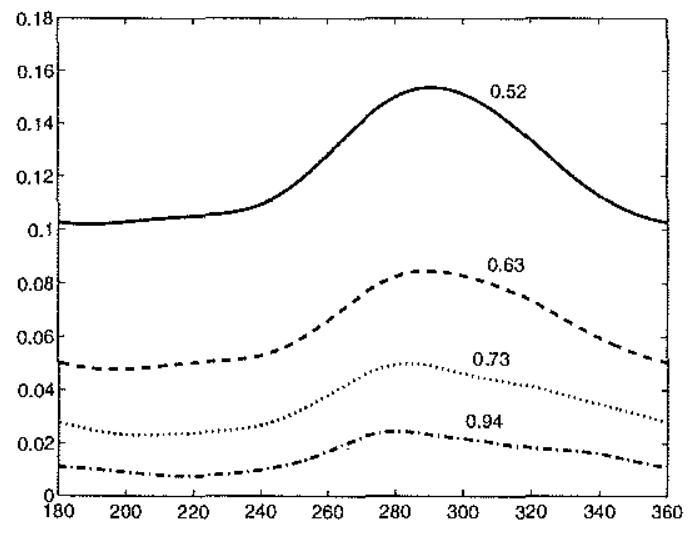

(d)

Figure 4-25: Directional Slices of Circular Wire Wave Gage Two-Dimensional Wavenumber Spectra from Data Sets 10281415, 10281445, and 10281515. The axes on subfigures (b) (d) are the same units as those in (a). Note that the y-axis scale of subfigure (b) is not the same as the others, however, and the $x$-axis has been shifted for (b) and (d). The numbers next to each curve represent the magnitude of the wavenumber at which the slice was taken and are given in $\mathrm{rad} / \mathrm{cm}$. Subfigures (a) and (b) are from data set 10281415, (c) is from data set 10281445 , and (d) is from data set 10281515 . 
the shape of this spectrum compares well with the shape of the slope spectrum estimated using the SLSG processing, then it can be said that the two methods, given the same input data, generate the same or similar estimates of the spectrum, thus validating the CWWG processing methods.

The Scanning Laser Slope Gage was developed in the early 90's by Dr. Erik Bock at the Woods Hole Oceanographic Institution and Dr. Tetsu Hara at the University of Rhode Island to measure the three-dimensional wavenumber-frequency slope spectrum of capillarygravity waves. It was designed to be deployed from a catamaran-type platform with two subassemblies: a laser pod which produces a collimated laser beam which is rotated in a circle producing a circular scan, and a head unit that measures the angular deflection of the incoming light beam using an array of photodiodes. The laser pod is located under the water with the beam centerline facing the air-water interface, and the head unit is lined up vertically above it. Both are mounted forward of the bow of the deployment platform. When the beam from the laser pod breaches the air-water interface it is refracted according to Snell's law. The angular deflection of the refracted beam is used to determine the surface slope at the point the beam left the water surface relative to the position of the SLSG. The result is a slope measurement at each successive position of the laser beam around the circle it traces. For the data presented in this section each scan was collected at $138.8 \mathrm{~Hz}$.

The three-dimensional spectrum is estimated by calculating the Fourier transform of the autocorrelation function, or covariance, of the surface slope. Using the same definitions as were used in equation 2.3 , the sea surface slope can be represented by

$$
\frac{\partial \eta}{\partial x}=s_{1}(x, y, t) \quad \frac{\partial \eta}{\partial y}=s_{2}(x, y, t)
$$

where $\eta=(x, t)=(x, y, t)$ is the sea surface displacement. The autocorrelation function of the $i$ th component of the surface slope can then be defined as

$$
R_{i}^{s l}(\xi, \zeta, \tau)=\overline{s_{i}(x, y, t) s_{i}(x+\xi, y+\zeta, t+\tau)}, \quad i=1,2
$$

From this definition, one can generate the two-sided, three-dimensional, wavenumber-frequency 
slope spectrum using the following relationship

$$
\Phi_{i}^{s l}\left(k_{1}, k_{2}, \omega\right)=\frac{1}{8 \pi^{3}} \iiint R_{i}^{s l} \exp \left[-i k_{1} \xi-i k_{2} \zeta-i \omega \tau\right] d \xi d \zeta d \tau, \quad i=1,2
$$

where the wavenumber vector is $k=(k \cos \theta, k \sin \theta)=\left(k_{1}, k_{2}\right)$, and $\omega$ is the wave frequency. The total slope spectrum is the sum of the two components,

$$
\Phi^{s l}\left(k_{1}, k_{2}, \omega\right)=\Phi_{1}^{s l}\left(k_{1}, k_{2}, \omega\right)+\Phi_{2}^{s l}\left(k_{1}, k_{2}, \omega\right)
$$

The two-sided wavenumber spectrum can be obtained by simply integrating the threedimensional spectrum in the frequency domain,

$$
\begin{aligned}
\Phi^{s l}(k, \theta) & =\int_{-\infty}^{\infty} \Phi^{s l}(k, \theta, \omega) d \omega \\
\Phi^{s l}\left(k_{1}, k_{2}\right) & =\int_{-\infty}^{\infty} \Phi^{s l}\left(k_{1}, k_{2}, \omega\right) d \omega
\end{aligned}
$$

For further information on the physical design and data analysis development for the SLSG please reference [8] from which the above description was taken.

The Fourier transform described in equation 4.5 cannot be calculated using straightforward fast Fourier transform (FFT) techniques for the same reason that the similar equation for estimating the circular wire wave gage two-dimensional surface height wavenumber spectrum cannot be: the data points in autocorrelation space are unevenly distributed. The circular wire wave gage processing overcomes this limitation by fitting a two-dimensional surface to the autocorrelation function and then estimating the spectrum. The processing algorithm used for the SLSG uses a different method. Since the SLSG data is sampled in evenly spaced increments in time, the temporal portion of the spectral integral is performed first using FFT methods. The resulting two-dimensional spatial integral is then numerically integrated using finite area elements associated with each lag coordinate. The finite areas are different for each spatial lag magnitude. A detailed description of this technique is given in the dissertation of Mete Uz [66] from the University of Rhode Island.

Two sets of data were obtained from Dr. Hara with the help of one of his graduate 
students, Hua Wei. The first data set is the fully processed three-dimensional slope spectrum in 6 minute segments from the CoOP '97 experiment. The second data set was composed of the calibrated time series slope data for the same 6 minute segments used to generate the first data set. To compare the processing algorithms, which will be referred to as SLSGproc and CWWGproc, the second data set was modified so as to allow it to be processed using the CWWGproc. The modifications included subsampling it spatially by using only every third point around the 15 centimeter diameter sampling circle used by the SLSG. The raw SLSG data is collected at 150 points around this circle, so the second data set was composed of only 50 sample points for each scan. This subsampling was necessary for two reasons, the first being to reduce the CWWGproc processing time which increases significantly as the number of points around the sampling circle becomes larger, and the second was to attempt to make the data set simulate something very similar to what the CWWG would have measured had it been actually sampling the identical wave field as the SLSG. The second data set was also bandlimited in frequency. This was done, again, to simulate CWWG conditions because during the CWWG calibration the measured wave heights are bandlimited before they are used to estimate the spectrum.

SLSG data from Julian day 198 in 1997 was processed with the SLSGproc algorithms and the resulting three-dimensional spectrum $\Phi^{s l}\left(k_{x}, k_{y}, \omega\right)$ was reduced to a two-dimensional spectrum by integrating out the frequency variable as explained in equation 4.8. The resulting slope spectrum representing a total of 12 minutes of sampling is shown in Figure 426. The same slope data was then processed using the CWWGproc algorithms after being filtered as described in the previous section. The resulting slope spectrum representing the same 12 minutes of data is shown in Figure 4-27. The wind velocity during this 12 minute segment of data collected by the SLSG was approximately $8.7 \mathrm{~m} / \mathrm{s}$ at 18 meters above the sea surface. When the total mean square slope (mss) was determined from each slope spectrum estimate by calculating the area underneath each spectrum, there was a significant discrepancy between the CWWGproc and SLSGproc results. The CWWGproc results gave a mss of 0.033 while the SLSGproc results gave a mss of $4.08 * 10^{-6}$. For the given wind speed during this data set of $8.3 \mathrm{~m} / \mathrm{s}$ at 10 meters $^{3}$ the expected mean square

\footnotetext{
${ }^{3}$ Adjusted from the 18 meter wind speed using the Charnock model.
} 

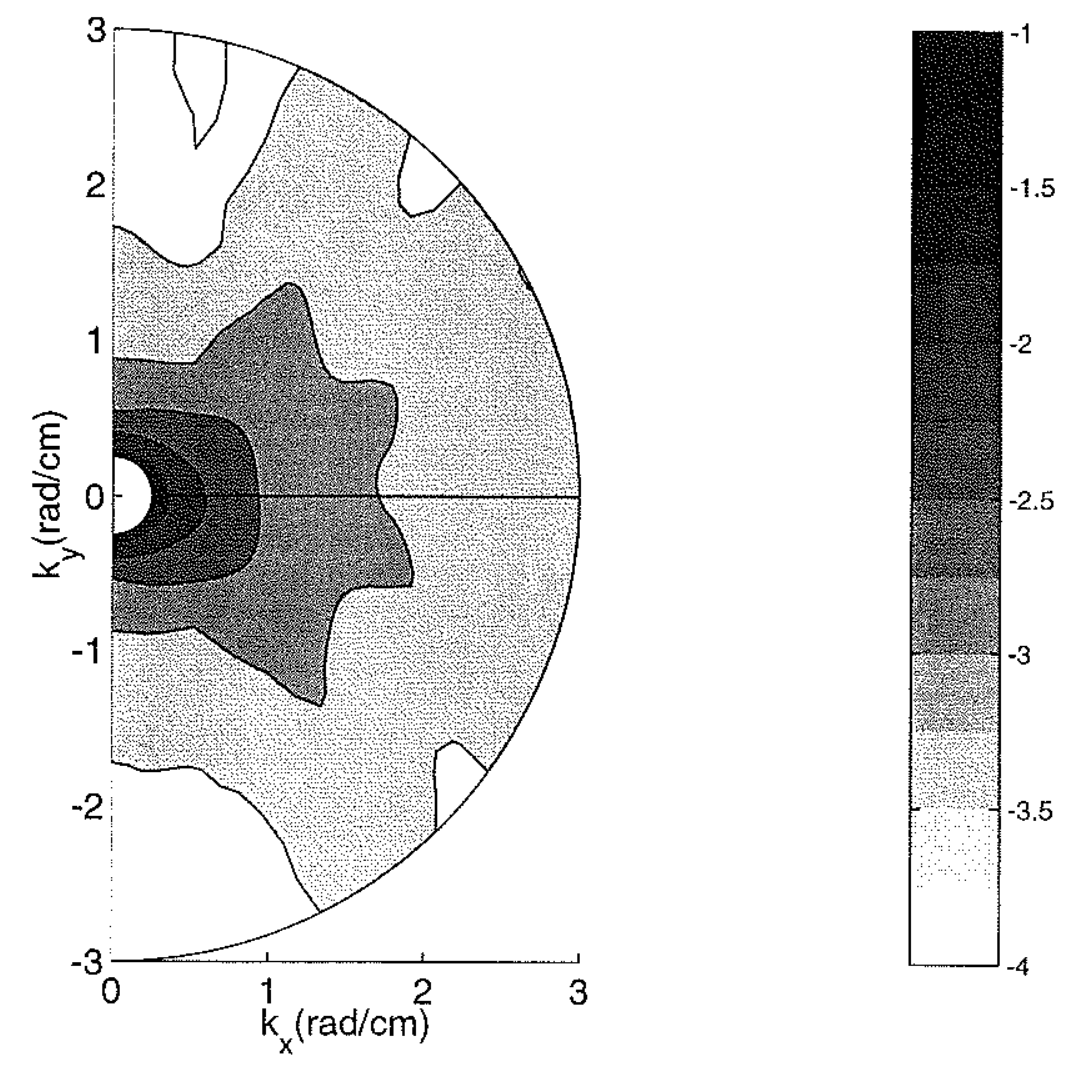

Figure 4-26: Scanning Laser Slope Gage Normalized Two-Dimensional Slope Spectrum Processed with SLSGproc from Data Sets ds023255.198 and ds023909.198. The spectrum is plotted as $\log _{10}\left(\Phi^{s l}\right)$. The $\Phi^{s l}$ units are in $(\mathrm{rad})^{2} /(\mathrm{rad} / \mathrm{cm})^{2}$. The magnitude of the spectrum was normalized as discussed in section 4.2 . 

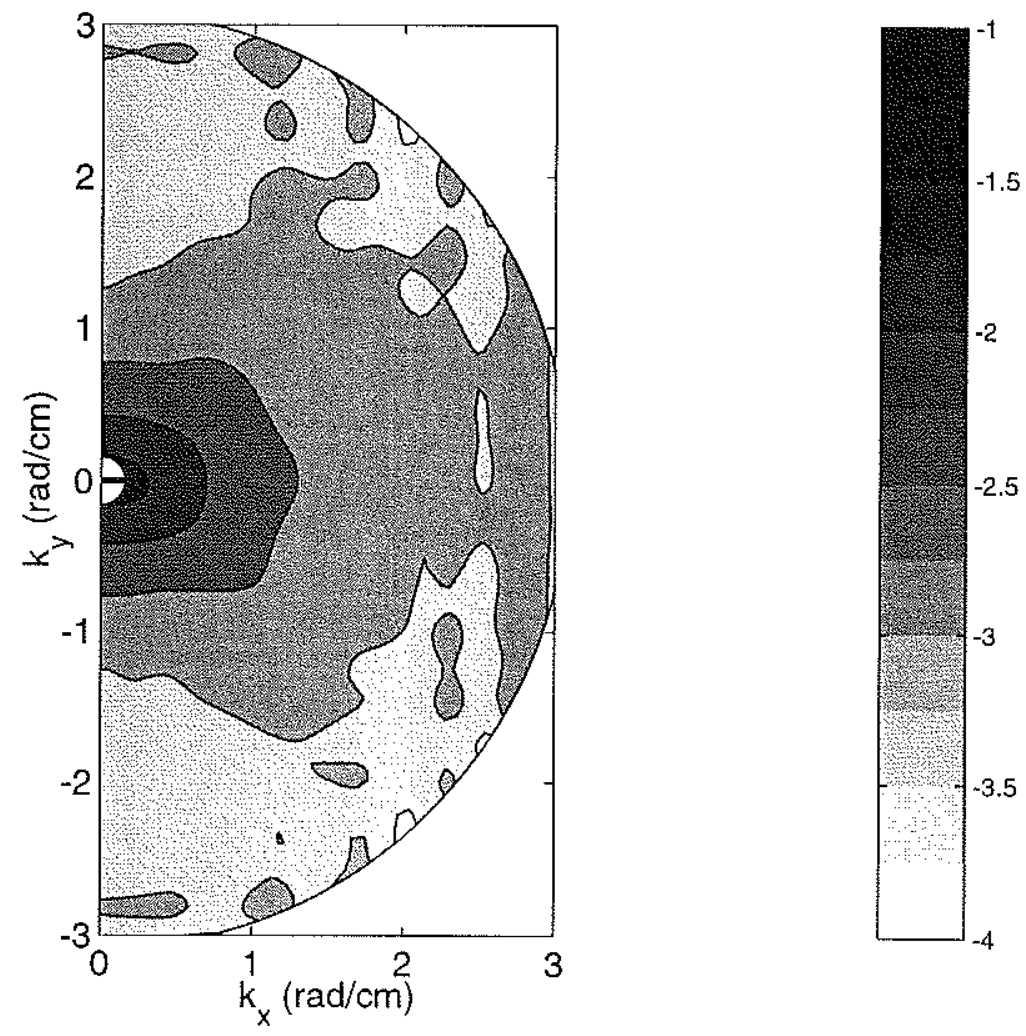

Figure 4-27: Scanning Laser Slope Gage Two-Dimensional Slope Spectrum Processed with CWWGproc from Data Sets ds023255.198 and ds023909.198. The spectrum is plotted as $\log _{10}\left(\Phi^{s l}\right)$. The $\Phi^{s l}$ units are in $(\mathrm{rad})^{2} /(\mathrm{rad} / \mathrm{cm})^{2}$. 
slope should be between approximately 0.03 and 0.06 [30]. After a discussion with Dr. Bock it was determined that the SLSGproc results contained a calibration error which accounted for the much lower than expected mean square slope value. This calibration error affects only the magnitude of the spectrum and not the angular distribution. Since the primary purpose of this processing method comparison was to assess the ability of the CWWGproc to accurately reproduce the directional characteristics of a spectrum, the magnitude of the spectrum generated by the SLSGproc algorithms was normalized. This normalization was implemented by integrating out the angular variable in both the CWWGproc and SLSGproc slope spectra resulting in a one-dimensional (1-D) slope spectrum as a function of wavenumber for each method. The SLSGproc 1-D spectrum was then multiplied by a constant to make the area underneath it equal to the area underneath the CWWGproc 1-D spectrum.

The two spectra shown in the logarithmic plots in Figures 4-26 and 4-27 are very similar (the logarithmic scale significantly enhances small differences). A closer look at their shapes is shown in Figure 4-28 which compares constant wavenumber slices from each of the processing methods. As can be seen, the CWWGproc at the lower wavenumbers does not appear to reproduce the narrowness of the spectrum which is evident in the SLSGproc results. It also seems to smooth the undulations visible in SLSGproc spectrum. This is expected given that the input data for the CWWGproc algorithms is a subset of the SLSGproc input data (only every third data point is used) so the angular resolution of the CWWGproc results should be less than for the SLSGproc results. Additionally, the smoothing window used in the CWWGproc data has a width of about $0.4 \mathrm{rad} / \mathrm{cm}$ which would also account for some smoothing of the spectrum.

The key purpose for comparing the shapes of the spectra using the two processing methods was to show that given the same input data, the two algorithms produced approximately the same result. The above figures show this to be generally true. It will be assumed, therefore, that based on this comparison, the CWWGproc algorithm for generating the two-dimensional spectrum is corroborated although the CWWGproc methods show a tendency to broaden the spectrum at low wavenumbers. 


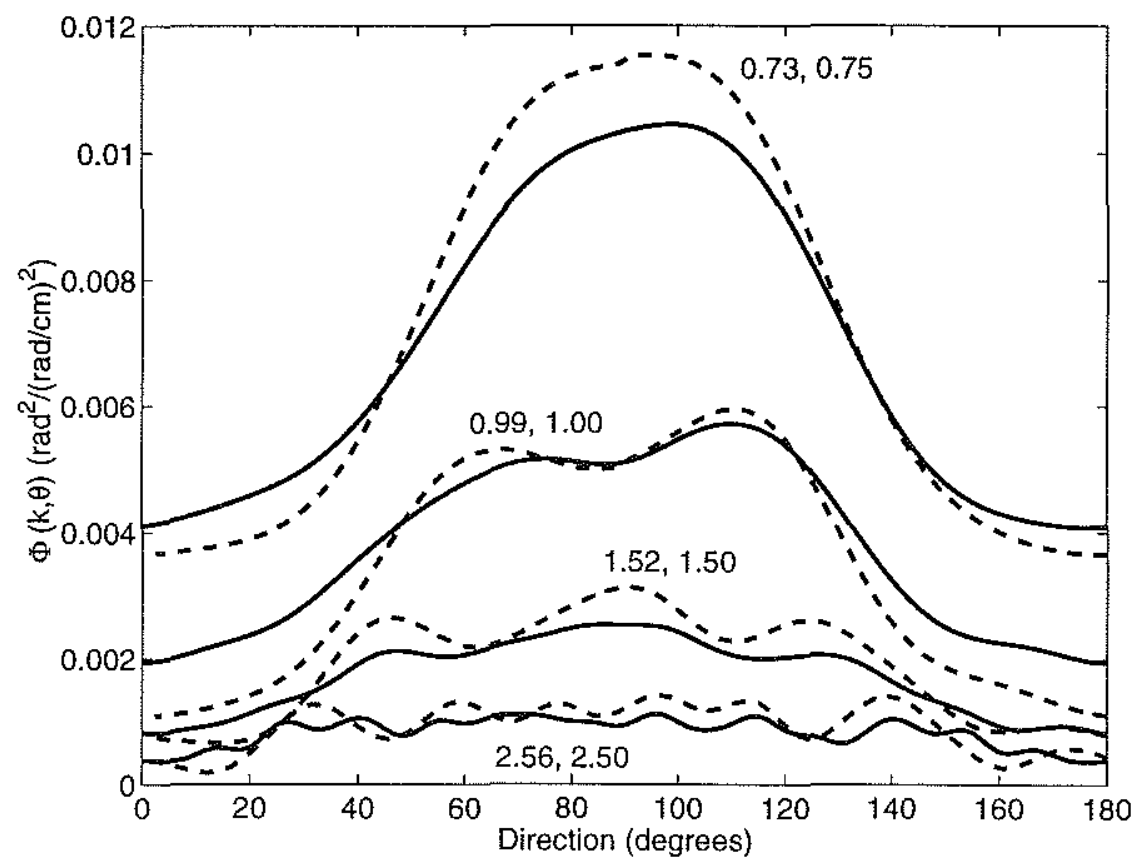

Figure 4-28: Directional Comparison Between SLSGproc and CWWGproc. The solid curves represent slices of the spectrum from the CWWGproc and the dashed curves represent slices of the spectrum from the SLSGproc. The two numbers next to each curve are the wavenumbers (in $\mathrm{rad} / \mathrm{cm}$ ) at which the slice is taken from the CWWGproc and SLSGproc spectra, respectively. The slices are not taken at the exact same wavenumbers because the spectral estimates are generated at a limited number of wavenumbers which are different for each processing method. 


\subsection{Discussion}

It is important to view the data presented in this chapter as a body of knowledge about wind and wave conditions at the Buzzards Bay Tower on October 28, 1998 (except for the last section about the scanning laser slope gage). Consistency in the data is therefore important. Evidence of this exists between the sonic anemometer measurements and the tower anemometer data: both show similar evolution of the wind direction over the course of the experiment from about $210^{\circ}$ to $225^{\circ} \mathrm{N}$. The scatterometer measurements also indicate a change in wind direction evidenced by the fact that the peak energy level shifts with the mean wind direction very consistently. Additionally, the long wave data measured by the capacitive wire wave gage indicates that the long wave energy is propagating primarily from the Southwest toward the Northeast. (It should be noted that in the meteorologic community wind direction is reported as the direction from which the wind is blowing, while in the surface wave community wave energy is most often reported as the direction in which the waves are propagating.) Finally, the single wire frequency spectra calculated from both the capacitive wire wave gage wires and the circular wire wave gage wires shows a remarkable match in both magnitude and slope over the ranges in which they both measure data as shown in Figure 4-29. This supports the accurate calibration of both instruments, as the frequency spectrum is a very reliable and consistent measure of ocean surface energy density. This also supports the assertion that the general statistical measures collected with the circular wire wave gage are the same as those collected at the tower even though they were not exactly co-located. The differences between the scatterometer directional measurements and the CWWG directional measurements will be discussed in the following chapter.

How this body of data both with its consistencies and questions relates to the current state of knowledge in the fields of short surface wave theory and ocean backscatter modeling will be discussed in the following chapter. 


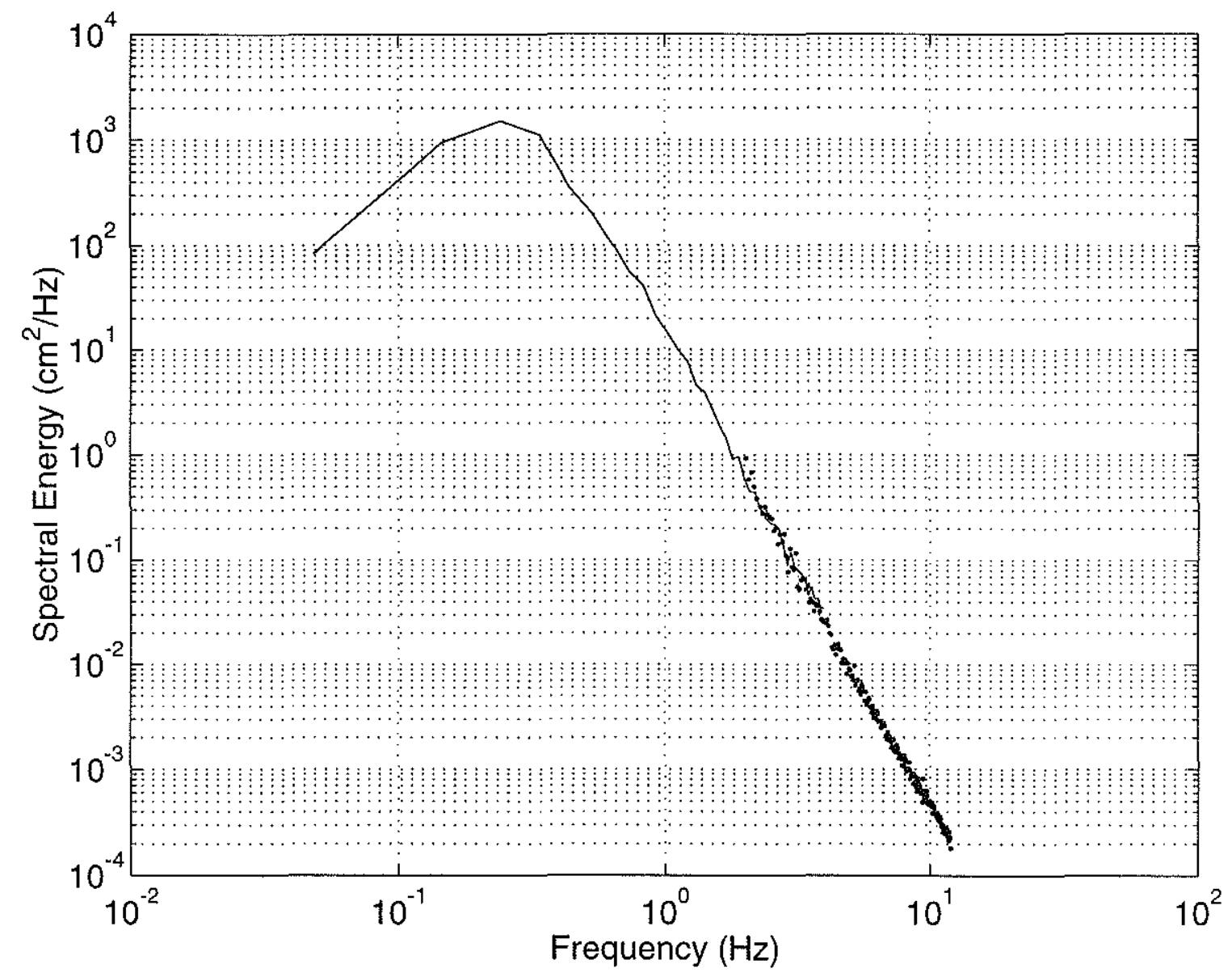

Figure 4-29: Comparison of the Frequency Spectrum as Measured by the Capacitive Wire Wave Gage and the Circular Wire Wave Gage. The solid line is the frequency spectrum from the capacitive wire wave gage data set 10281414 (see Figure 4-13). The dots are the frequency spectrum from the circular wire wave gage data set 10281515 (see Figure 4-20). 


\section{Chapter 5}

\section{The Wave Height Spectrum and Scatterometry}

The data presented in the last chapter offer a unique opportunity to study and compare in situ measurements of high-resolution two-dimensional wavenumber spectra and electromagnetic backscatter return collected co-temporally. No currently published experiment or study has accomplished this. The results from this experiment are mixed. The scatterometer data compares well with current theories and other scatterometer measurements, but the circular wire wave gage two-dimensional spectrum results compare favorably with only some of the current spectral models. And with respect to the angular alignment of the wave gage energy distribution with the wind direction, the data is inconsistent with the expectation that the wind and short waves are generally well aligned. This chapter will focus on comparing the separate pieces of the Buzzards Bay Tower data with current models, and will then treat the data as a collective unit to compare with models which attempt to use portions of the data (such as a two-dimensional wavenumber spectrum) as input to more complex models (such as electromagnetic scattering models); the goal being to explain as much of the deviation from the current theories as possible. 


\subsection{The Wave Height Spectrum}

Frequency and wavenumber representations of the surface wave height spectrum provide similar information about the energy distribution of surface waves, but they are collected and often modeled in different ways, especially in the gravity-capillary region of the spectrum where Doppler shifting makes transformations between the two representations ambiguous. This section is therefore broken into two subsections, the frequency spectrum and the wavenumber spectrum, in order to show the evolution of current models in each area.

\subsubsection{Frequency Spectrum}

The omnidirectional frequency spectrum is one of the easiest statistical descriptions of surface wave height to determine because it can be calculated from a single time series of surface wave height measurements. Phillips [48] was the first to present a widely accepted model for the analytical form of this spectrum. He theorized that if the duration and fetch of the wind are large, then the smaller-scale components of the wind-generated wave field may be in a condition of equilibrium determined by the requirements for attachment of the crests of the waves. His model is based on a dimensional analysis of this idea of an equilibrium range and is valid for the rear face (high frequency portion) of the spectrum. It takes the form

$$
S(\omega) \sim \alpha g^{2} \omega^{-5}, \quad \omega_{p e a k} \ll \omega \ll \frac{2 g}{u_{*}}
$$

where $g$ is the acceleration due to gravity, $\omega$ is frequency in $\mathrm{rad} / \mathrm{s}, u_{*}$ is the wind friction velocity in $\mathrm{m} / \mathrm{s}$, and $\alpha$ is a parameter independent of $\omega$ and environmental conditions, which is obtained by fitting experimental data.

While data at the time appeared to substantiate this theory, further study indicated that $\alpha$ actually depended in some manner on other parameters such as fetch and wind speed or friction velocity. Pierson and Moskowitz [49] extended the Phillip's spectrum to cover the long wave portion of the spectrum and modeled it after their own data for fully 
developed seas:

$$
S(\omega)=\alpha g^{2} \omega^{-5} \exp \left(-\beta\left(\omega_{0} / \omega\right)^{4}\right), \quad \omega_{0}=g / U
$$

where $U$ is the wind speed at 19.5 meters above the sea surface and $\alpha$ and $\beta$ are constants to be determined. The next major advance came with the analysis of data from the Joint North Sea Wave Project (JONSWAP) conducted in 1968 and 1969 which included fetch-limited seas. The resulting analysis prompted the modification of the Pierson-Moskowitz spectrum by multiplying it by a 'peak enhancement' factor $\gamma^{\delta}$ [27] where $\gamma$ is a fitted parameter describing the degree of peakedness and $\delta$ is an exponential dependent on frequency (a variable), peak frequency of the spectrum (a constant), and a fitting parameter $\sigma_{0}$ which describes the width of the peak region.

It had been accepted by this time that the spectrum was a function of some combination of energy balancing factors such as input energy from wind, energy transfer between waves of different wavelength, and viscous dissipation. Most models after this time include some form of fetch or wave age dependence. Continuing analysis and at-sea experimentation in the 60's and 70's lead to a more fundamental modification of the basic Phillips spectrum, however. Much of this newer data supported a slope of $\omega^{-4}[62,46,6]$. Donelan, Hamilton, and Hui [17] (DHH) provided the next wide-reaching and in-depth study of surface waves based on both lake (Lake Ontario) and laboratory (CCIW) experimentation in the late 70's. Their conclusions have been widely accepted as the basis upon which current models are tested. Their study gave strong evidence to support the newer $\omega^{-4}$ power law and was used to generate a model which modified the JONSWAP spectrum to account for the different power law. It takes the form

$$
S(\omega)=\alpha g^{2} \omega^{-4} \omega_{\text {peak }}^{-1} \exp \left[-\left(\frac{\omega_{\text {peak }}}{\omega}\right)^{4}\right] \gamma^{\Gamma}
$$

with

$$
\Gamma=\exp \left[-\left(\omega-\omega_{p e a k}\right)^{2} / 2 \sigma^{2} \omega_{p e a k}^{2}\right]
$$

where the four parameters of the model are $\omega_{\text {peak }}$, the peak frequency of the spectrum, $\alpha$, 
a fitted rear face variable, $\gamma$, a fitted peak enhancement factor, and $\sigma$, a fitted peak width variable. Donelan et al. [17] also give approximate relationships between the fitted variables and the phase speed at the peak of the spectrum as well as the wind speed.

An example of the frequency spectrum measured by the circular wire wave gage (CWWG) and the capacitive wire wave gage is compared with the Donelan, Hamilton, and Hui spectrum in Figure 5-1. It is evident from this comparison that the measured spectrum near the spectral peak is closer to the $\mathrm{DHH} \omega^{-4}$, but at frequencies higher than about $1 \mathrm{~Hz}$ the slope increases to near $\omega^{-5}$. It should be noted, however, that the DHH work only modeled the spectrum up to frequencies approximately three times the spectral peak and the extension out to much higher frequencies was done for illustrative purposes only. It stands to reason

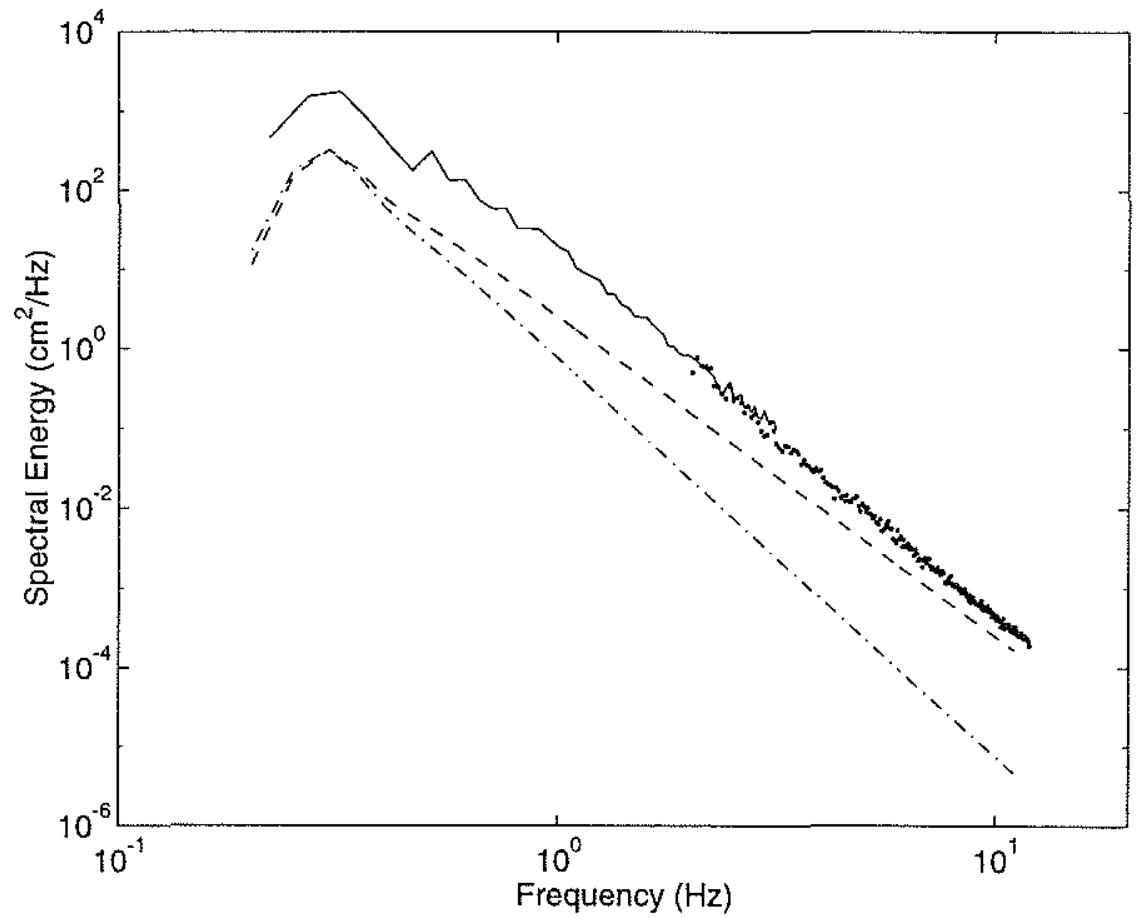

Figure 5-1: Comparison of the DHH Frequency Spectrum Model with a Frequency Spectrum Measured at the Buzzards Bay Tower. The solid line is the frequency spectrum from the capacitive wire wave gage data set 10281315 (see Figure 4-13). The dots are the frequency spectrum from the circular wire wave gage data set 10281415 (see Figure 4-24). The dashed line is the DHH spectral model given in equation 5.3. The dash-dot line is the DHH spectrum modified to use an $\omega^{-5}$ rear face slope (similar to the original JONSWAP spectral model). 
that the resulting difference between the measured spectrum and the DHH model simply indicates that at the higher frequencies, other factors besides wind speed and the peak phase speed (which are used as indicators of wave age) may be affecting the spectrum, or that the energy balances may be different. From a dimensional standpoint, if the slope of the measured omnidirectional wavenumber spectra of -2.5 (shown in Figure 4-24) is transformed into frequency space using the dispersion relation and no Doppler shifting, the resulting frequency spectrum would have a slope of $\omega^{-4}$, which indicates that some of the steepening in the frequency spectrum may be the result of Doppler shifting, although there are other physical mechanisms at these frequencies that could affect the spectral slope including wind drift and ambient current [17]. There have been convincing arguments and measurements in the literature, however, that support a frequency spectral slope of somewhere between -4 and -5 , but the limited amount of wavenumber and meteorological data available with this data set do not allow for suggested modifications to or specific support of any current models except in the matter of the overall slope of the frequency spectrum.

Most of the frequency spectrum models developed after the mid-80's were not based on direct measurements of the frequency spectrum, but were produced using indirect methods involving transformations or integrations of either a one- or two-dimensional wavenumber spectrum or a more complex three-dimensional spectrum $(\Phi(k, \omega))$. These models are based on measurements or analytical development in wavenumber space, so they will be presented in the following section.

\subsubsection{One- and Two-Dimensional Wavenumber Spectra}

It has been more difficult to obtain experimental measurements of the wavenumber spectrum due to the need for extensive spatial simultaneous sampling of wave elevations. As a result, the earliest models of the wavenumber spectrum were generated by transforming a frequency spectrum model using some form of the dispersion relation. The problem with this method arises at high frequencies due to Doppler shifting of the short waves components by the long wave orbital velocities causing transformations to become ambiguous. Instrumentation has been developed over the last 10 or so years which has finally allowed the wavenumber spectrum at higher frequencies to be measured directly, which has lead to more robust 
wavenumber spectrum models. The circular wire wave gage discussed in this thesis is one such instrument.

There are two commonly used forms for describing the two-dimensional wavenumber spectrum, the direct form and the spreading function form. The direct form itself has two representations: $\Phi\left(k_{x}, k_{y}\right)=\Phi(k)$ and $\Phi(k, \theta)$. The total mean square wave height energy, $\overline{\eta^{2}}$, can be obtained from either form using the following equation

$$
\overline{\eta^{2}}=\int_{-\infty}^{\infty} \int_{-\infty}^{\infty} \Phi(k) d k=\int_{-\pi}^{\pi} \int_{0}^{\infty} \Phi(k, \theta) k d k d \theta
$$

where $k=\left(k_{x}, k_{y}\right)$ is the wavenumber vector, $k=|k|$ is the magnitude of the wavenumber vector, and $\theta$ is the angle of the vector $k$. The omnidirectional spectrum shall be defined as

$$
S(k)_{d e f}=\int_{-\pi}^{\pi} \Phi(k, \theta) k d \theta
$$

describing the wave height spectral density as a function of wavenumber, $k$, for all directions. The spreading function form of representation is:

$$
\Phi(k, \theta)=\Phi\left(k, \theta_{\max }\right) D(k, \theta)
$$

where $D(k, \theta)$ is a non-dimensional spreading function used to model the azimuthal distribution of energy and is conventionally presented with $D(k, 0) \equiv 1$ for all $k{ }^{1}$ where the wind is assumed to be sufficiently steady so that the dominant wind waves are aligned with the mean wind direction $(\theta=0)$. The function $\Phi\left(k, \theta_{\max }\right)$ will be called the "wavenumber spectral slice in the peak energy direction' in this text and represents the distribution of energy purely as a function of wavenumber. A number of directional spreading functions, $D(k, \theta)$ were described in detail in Chapter 2, but for thoroughness Tables 5.1 and 5.2 list these spreading functions again and include their associated high frequency wavenumber spectral slice models, $\Phi\left(k, \theta_{\max }\right)$.

The two-dimensional wave height spectra generated using the CWWG data represent the directional distribution of energy in a purely spatial domain (lacking any fre-

\footnotetext{
${ }^{1}$ This definition is different from spreading functions which require a unity polar integral.
} 
Year Reference

1990 Banner [3]

1994 Apel [1]

1996 Caudal et al. [9]
Spreading Function

$$
\begin{aligned}
& D(k, \theta)=\cosh ^{-2}\left[\beta\left(\theta-\theta_{\max }\right)\right] \\
& \beta= \begin{cases}2.28\left(k / k_{p}\right)^{-0.65}, & 0.97<k / k_{p}<2.56 \\
10^{-0.4+0.839 \exp \left[-0.567 \ln \left(k / k_{p}\right)\right]}, & k / k_{p}>2.56\end{cases}
\end{aligned}
$$

$$
\begin{gathered}
D(k, \theta)=\exp \left\{-\left(\theta-\theta_{\text {upwind }}\right)^{2} /\left[2 \theta_{s}^{2}(k)\right]\right\} \\
1 /\left[2 \theta_{s}^{2}(k)\right]=0.14+5.0\left(k / k_{p}\right)^{-1.3}
\end{gathered}
$$

$$
\begin{aligned}
D(k, \theta) & =\exp \left(-\alpha \theta^{2}\right) \\
\alpha & =0.14+5.0\left(k / k_{p}\right)^{-1.3}+\delta, \quad 0.9<k<1180 \\
\delta & =10^{(-0.029 U+0.064) X^{2}+(0.127 U+0.050) X+(-0.126 U-0.930)} \\
X & =\log _{10}(k)
\end{aligned}
$$

1997 Elfouhaily et al. [19] ${ }^{a} \quad D(k, \theta)=(2 \pi)^{-1}[1+\Delta(k) \cos (2 \theta)]$

$$
\Delta(k)=\tanh \left[0.173+4\left(c / c_{p}\right)^{2.5}+0.565 u^{*}(0.23 / c)^{2.5}\right]
$$

1997 Romeiser et al. [55] $\quad D(k, \theta)=\exp \left[-\theta^{2} /\left(2 \delta^{2}\right)\right]$

$$
\begin{gathered}
1 /\left(2 \delta^{2}\right)=0.14+0.5[1-\exp (-k U / 400)]+ \\
5 \exp [2.5-2.6 \ln (U)-1.3 \ln (k)]
\end{gathered}
$$

Table 5.1: Directional Spreading Functions from Various Models. $k$ is the wavenumber in $\mathrm{rad} / \mathrm{m}, k_{p}$ is the wavenumber at the peak of the wave height spectrum in $\mathrm{rad} / \mathrm{m}, U$ is the wind speed at 10 meters above the water surface in $\mathrm{m} / \mathrm{s}, c$ is the phase speed in $\mathrm{m} / \mathrm{s}, c_{p}$ is the phase speed at the peak of the wave height spectrum in $\mathrm{m} / \mathrm{s}, \theta$ is the azimuthal direction in radians, and $u^{*}$ is the friction velocity in $\mathrm{m} / \mathrm{s}$.

\footnotetext{
${ }^{a}$ The Elfouhaily spreading function model does not conform to the typical definition for the spreading function given in equation 5.6 in the sense that $D(k, 0) \neq 1$ for all $k$. Elfouhaily does choose, however, to compare his (non-normalized) spreading function and the resulting omnidirectional spectrum (as defined in equation 5.5) to other spreading functions and omnidirectional spectra in the literature which do require $D(k, 0)=0$, so for the purposes of comparison herein, the model he presents in his paper will be used unchanged.
} 
Year Reference Wavenumber Spectral Slice in the Peak Energy Direction

1990 Banner $[3] \quad \Phi\left(k, \theta_{\max }\right)=0.45 \times 10^{-4}\left(U / c_{p}\right)^{1 / 2} k^{-4}, \quad k$ in cycles $/$ meter

1994 Apel [1] $\Phi\left(k, \theta_{\max }\right)=0.00195 \exp \left[-\left(k_{p} / k\right)^{2}\right] J_{p} H_{i} k^{-4}$

$$
\begin{aligned}
& J_{p}=1.7^{\exp \left[-\left(\sqrt{k}-\sqrt{k_{p}}\right)^{2} /\left(0.32 k_{p}\right)\right]} \\
& H_{i}=\left\{\left[1+(k / 100)^{2}\right]^{-1}+\exp \{\ln [-49.5+34.5(1-\exp (-U / 4.7))]\}\right. \\
& \left.0.8 k \cosh [(k-400) / 450]^{-1}\right\} \exp \left(-k^{2} / 6283^{2}\right)
\end{aligned}
$$

1997 Elfouhaily $\Phi\left(k, \theta_{\max }\right)=\left[B_{l}+B_{h}\right] k^{-4}$

et al. [19]

$$
\begin{aligned}
& B_{h}=0.00115\left[1+3 \ln \left(u^{*} / 0.23\right)\right] c^{-1} \exp \left[-0.25(k / 371-1)^{2}\right] \\
& B_{l}=0.003 \sqrt{U / c_{p}} c_{p} / c \exp \left[-1.25\left(k_{p} / k\right)^{2}\right] \\
& \quad\left[1.7+6 \log \left(U / c_{p}\right)\right]^{\exp \left\{-\left[\sqrt{k / k_{p}}-1\right]^{2}\left\{0.16+0.64\left(U / c_{p}\right)^{-3}\right]^{-1}\right\}} \\
& \quad \exp \left[-0.316 U / c_{p}\left(\sqrt{k / k_{p}}-1\right)\right]
\end{aligned}
$$

1997 Romeiser

$$
\Phi\left(k, \theta_{\max }\right)=P_{L}(k, U) W_{H}(k) U^{\beta(k)} k^{-4}
$$

et al. [55]

$$
\begin{aligned}
& P_{L}=0.000195 \exp \left\{-\left(k_{p}^{2} / k^{2}\right)+0.53 \exp \left[-\left(\sqrt{k}-\sqrt{k_{p}}\right)^{2} /\left(0.32 k_{p}\right)\right]\right\} \\
& W_{H}=\left[1+(k / 280)^{7.2}\right]^{0.5}\left[1+(k / 75)^{2.2}\right]^{-1}\left[1+(k / 1300)^{3.2}\right]^{-2} \\
& \quad \exp \left(-k^{2} / 8885^{2}\right) \\
& \beta=\left[1-\exp \left(-k^{2} / 183^{2}\right)\right] \exp (-k / 183)+ \\
& \quad[1-\exp (-k / 33)] \exp \left\{-[(k-140) / 220]^{2}\right\}
\end{aligned}
$$

Table 5.2: High Frequency Wavenumber Spectral Slice in the Peak Energy Direction from Various Models. $k$ is the wavenumber in $\mathrm{rad} / \mathrm{m}$ except where noted, $k_{p}$ is the wavenumber at the peak of the wave height spectrum in $\mathrm{rad} / \mathrm{m}, U$ is the wind speed at 10 meters above the water surface in $\mathrm{m} / \mathrm{s}, c$ is the phase speed in $\mathrm{m} / \mathrm{s}, c_{p}$ is the phase speed at the peak of the wave height spectrum in $\mathrm{m} / \mathrm{s}, \theta$ is the azimuthal direction in radians, and $u^{*}$ is the friction velocity in $\mathrm{m} / \mathrm{s}$. 
quency/temporal information); so the spectral energies in directions differing by $180^{\circ}$ are the same. Guissard [22] considers this and explains that there are actually two possible interpretations of the notion of a spectrum which lead to different functions that do not have the same angular behavior: one is symmetric (the wave height spectrum), the other is not. He continues on to explain that for the purposes of electromagnetic scattering models, although there are often upwind-downwind asymmetries in backscattered return, this difference cannot be attributed to the contribution of the spectrum through linear Bragg scattering mechanisms. This is because these mechanisms are directly determined by the wave height spectrum which is, as defined, symmetric. So the alternate (non-symmetric) form of the spectrum, while useful, is not appropriate for use in linear scattering models. ${ }^{2}$ For the purposes of consistency in the spectral comparisons between the CWWG data and the models, therefore, only the (symmetric) wave height spectrum will be used. The spreading models presented in Table 5.1 are not constrained to be symmetric, though, so a symmetrized or folded spreading function is used instead. This folded spreading function is defined as

$$
D_{s}^{*}(k, \theta)=0.5[D(k, \theta)+D(k, \theta-\pi)]
$$

where the spreading functions $D(k, \theta)$ are those given in Table 5.1. Finally, to permit effective comparison between the models and the CWWG data, since the resulting folded spectra of the previously non-symmetric models will have somewhat smaller magnitudes at $D_{s}^{*}(k, 0),{ }^{3}$ all of the spreading models and the CWWG azimuthal slice data have been normalized as a function of $k$ by their upwind magnitude for consistency with the previous convention that $D(k, 0)=1$. This normalized spreading function will be defined as

$$
D_{s}(k, \theta)=D_{s}^{*}(k, \theta) / \max \left[D_{s}^{*}\left(k, \theta_{\max }\right)\right]
$$

\footnotetext{
${ }^{2}$ Guissard does offer some possible non-Bragg mechanisms to explain the upwind-downwind asymmetry in his paper. It should be noted that some very recent and more complicated scattering models than are presented in this thesis do attempt to incorporate a method to account for this asymmetry while still including the symmetric wave height spectrum dependencies required for linear Bragg scattering theory.

${ }^{3}$ This is true because the 'upwind' magnitude of $D(k, \theta)$ is constrained to be equal to 1 , but the 'downwind' magnitude of $D(k, \theta)$ is much less than 1 (i.e. the non-symmetry) so the sum of the 'upwind' and 'downwind' components multiplied by $1 / 2$ will be less than 1 .
} 
where the maximum is calculated for each value of $k$ and occurs when $\theta_{\max }=0$ for each of the models and where $\theta_{\max }=$ the maximum of the measured wave height spectrum for the circular wire wave gage data.

Figures 5-2, 5-3, and 5-4 compare the omnidirectional spectrum and wavenumber spectral slice models with the data collected with the circular wire wave gage. The Banner model is not shown in either the two-dimensional spectral slice plot or the omnidirectional plot because it is nearly identical to the Apel model. The omnidirectional spectra calculated from the models were evaluated numerically using

$$
S(k)=\int_{-\pi}^{\pi} \Phi\left(k, \theta_{\max }\right) D_{s}(k, \theta) k d \theta
$$

A Caudal model spectrum in the dominant wind direction is not listed in the table or shown in the plots because Caudal et al. did not propose their own form of the spectrum, instead they suggested using Apel's $\Phi\left(k, \theta_{\max }\right)$, which is what was used to calculate the $S(k)$ integral which is shown for the Caudal model. Finally, the CWWG data has a resolution down to approximately $0.2 \mathrm{rad} / \mathrm{cm}$ due to instrument and processing constraints. The windowing used during the processing, therefore, causes the slight dip in the spectra near the low wavenumber cutoff.

It is apparent from Figures 5-2 through 5-4 that the Romeiser et al. model comes closest to accurately describing the spectral values calculated from the circular wire wave gage data both in magnitude and slope. Interestingly, at wavenumbers above about 1 or 2 $\mathrm{rad} / \mathrm{cm}$ the CWWG spectral slice data begins to reduce its slope. This could be explained a number of ways. Banner [3] presented some analytical results indicating that the slope of the spectrum near the spectral peak can reduce the slope of the high frequency end of the spectrum through long wave spatial (wavelength) modulation of the short waves. He also showed that ambient surface currents can have the same effect. Thus, given the likelihood of strong currents in the location of the experiment, there is a possibility that the surface current velocities were larger than the phase velocities of the short waves, leading to what may be a significant effect on the expected spectral slope and energy distribution. Another explanation could be increased instrumentation error at these smaller amplitude waves. 

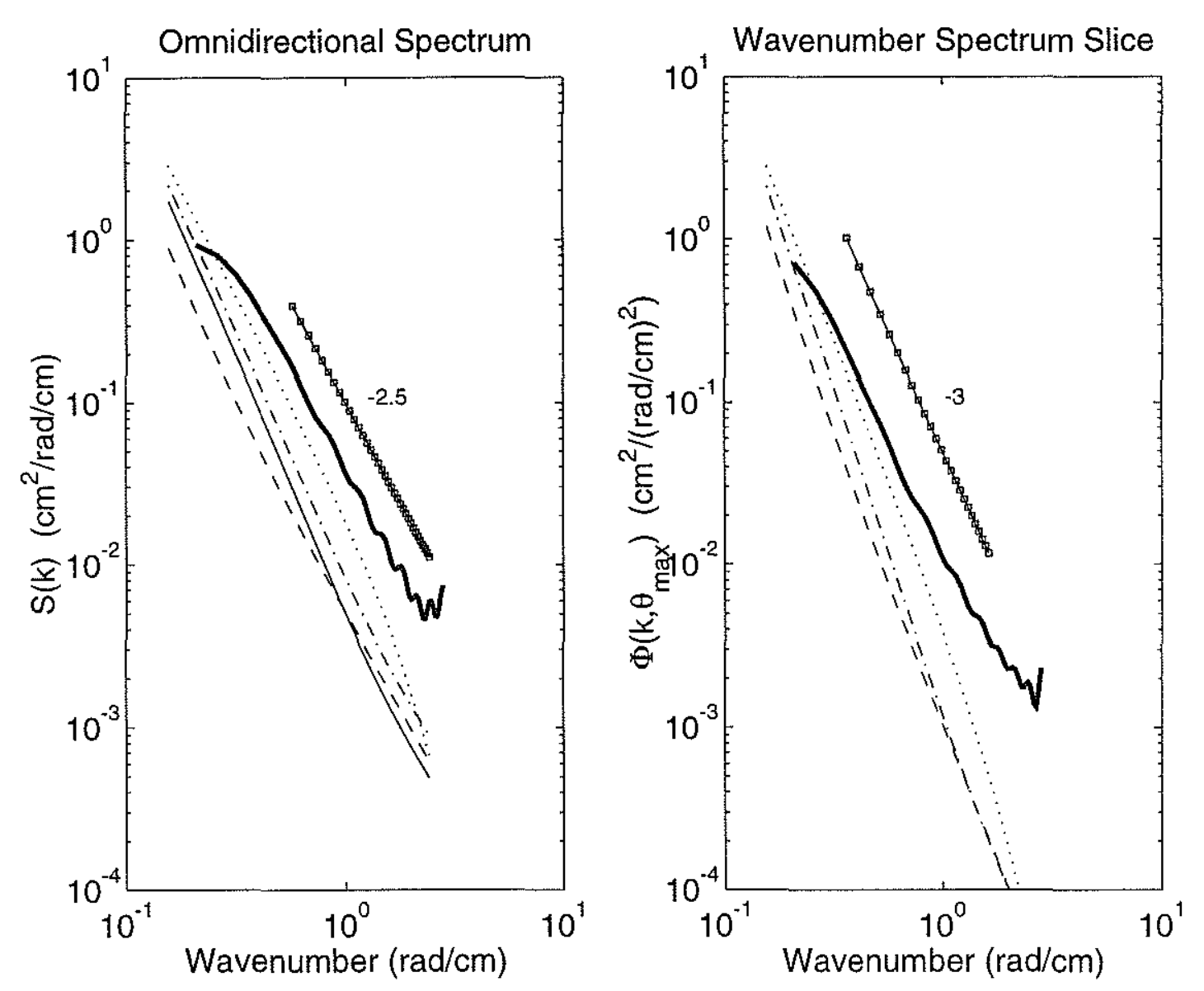

Figure 5-2: Comparison of the Omnidirectional Spectrum Models and Wavenumber Spectrum Slice Models with Data Collected at the Buzzards Bay Tower (10281415). The thick solid line is a slice of the two-dimensional wavenumber frequency spectrum in the maximum spectral direction as measured with the circular wire wave gage (CWWG). The CWWG measurements are averaged over 10 minutes for the omnidirectional spectrum, and 20 minutes for the spectral slice. The thin lines represent the model functions: ....... $=$ Apel, $\cdots=$ Elfouhaily et al., and $\cdots=$ Romeiser et al. The thin solid line in the omnidirectional spectrum plot is the Caudal et al. model. The solid line with the small square data points is a reference curve for each plot showing a particular wavenumber slope. 

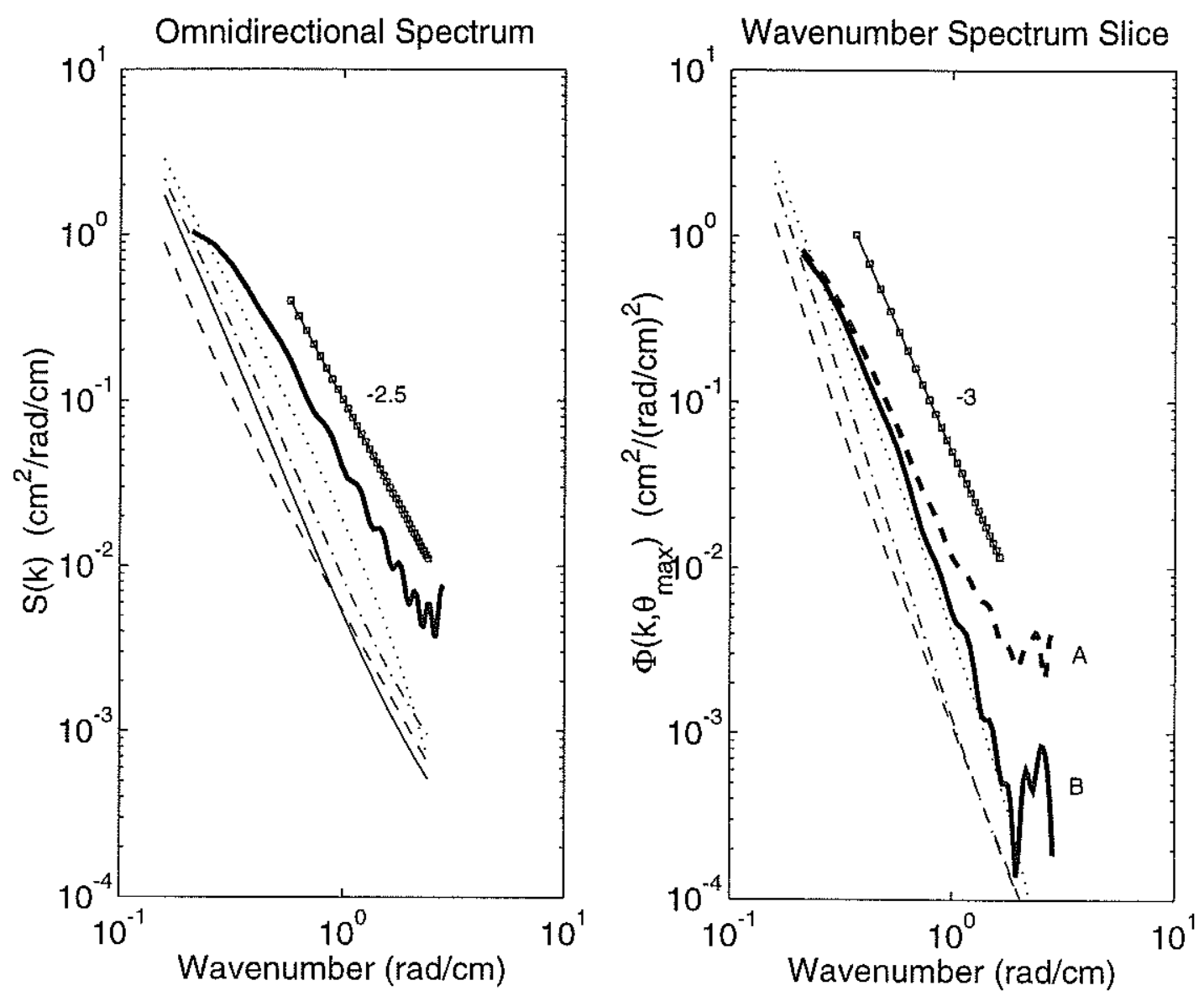

Figure 5-3: Comparison of the Omnidirectional Spectrum Models and Wavenumber Spectrum Slice Models with Data Collected at the Buzzards Bay Tower (10281445). The thick solid and thick dashed lines are slices of the two-dimensional wavenumber frequency spectrum as measured with the circular wire wave gage (CWWG). The solid line (B) is a slice in the mean wind direction, and the dashed line $(\mathrm{A})$ is a slice in the maximum spectral direction. The CWWG measurements are averaged over 10 minutes for the omnidirectional spectrum, and 20 minutes for the spectral slice. The thin lines represent the model functions: $-\cdots=$ Apel, $-\cdots=$ Elfouhaily et al., and $\cdots=$ Romeiser et al. The thin solid line in the omnidirectional spectrum plot is the Caudal et al. model. The solid line with the small square data points is a reference curve for each plot showing a particular wavenumber slope. 

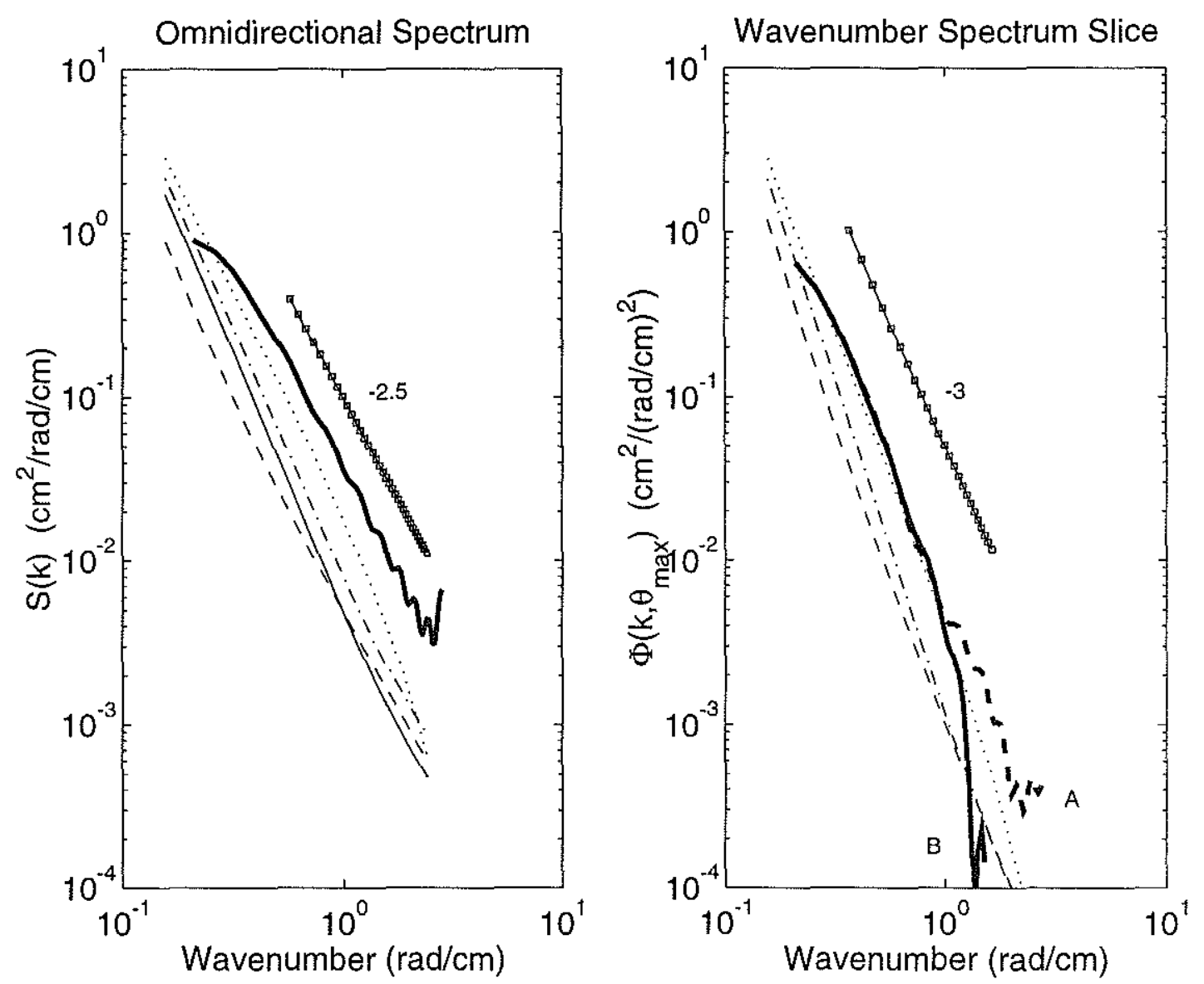

Figure 5-4: Comparison of the Omnidirectional Spectrum Models and Wavenumber Spectrum Slice Models with Data Collected at the Buzzards Bay Tower (10281515). The thick solid and thick dashed lines are slices of the two-dimensional wavenumber frequency spectrum as measured with the circular wire wave gage (CWWG). The solid line (B) is a slice in the mean wind direction, and the dashed line $(A)$ is a slice in the maximum spectral direction. The CWWG measurements are averaged over 10 minutes for the omnidirectional spectrum, and 20 minutes for the spectral slice. The thin lines represent the model functions: $\cdots \cdot \cdots=$ Apel, $\cdots=$ Elfouhaily et al., and $\cdots=$ Romeiser et al. The thin solid line in the omnidirectional spectrum plot is the Caudal et al. model. The solid line with the small square data points is a reference curve for each plot showing a particular wavenumber slope. 
The high wavenumber tail of the spectral slice in the maximum spectral direction (as well as those in the mean wind direction) shift between 20 minute data sets in a nonuniform manner, however, indicating that instrumentation noise is probably not entirely responsible for the variations. The Elfouhaily et al. model is the only other model shown which comes close to reproducing the spectral slopes of the at sea data. Interestingly, and not yet interpreted, is that the Romeiser model is based on fitting a model to radar backscatter data, while the Elfouhaily model is based on wave tank measurements. The Apel model is based on both scatterometer and tank data, but is valid only for fully developed seas, so it is not unexpected that it generates too steep a spectral slope. Likewise, the Caudal model is also too steep, and while it was based on scatterometer data, it is not limited to only fully developed seas. Another reason for the slope inconsistencies between the CWWG data and the models in the spectral slice graphs may be the sensitivity that the models have to the location of the spectral peak. The frequency of the spectral peak at the Buzzards Bay Tower, found using the capacitive wire wave gage, may differ from the true frequency of the spectral peak by up to $5 \%$ because the location of the spectral peak is a random variable which is generally under sampled (especially during limited duration experiments such as this one) as was explained by Donelan and Pierson in 1983 [16]. The key information in these plots is that the CWWG omnidirectional spectra support the -2.5 to -3 slopes suggested in the current models, but that the CWWG wavenumber spectral slices support a slope of -3 which is smaller than the -4 model slopes.

Figures 4-21, 4-22, and 4-23 from Chapter 4 show that the wind and the peak energy of the measured wave height spectra are not always well aligned. To see this in detail, Figures 5-5, 5-6, and 5-7 show slices of the two-dimensional wavenumber spectrum at a constant wavenumber (resulting in a circular slice of the 2-D wavenumber spectrum) for both the non-dimensional normalized form of the spreading models, $D_{s}(k, \theta)$, and the normalized CWWG data. In all of these plots the peaks of the spreading models are centered on the mean wind direction, which is their standard orientation, while the data is presented as measured.

These plots show a significant shifting of the peak location over the course of the experiment. Although the central measure studied in this thesis is the spectral width, a digression 

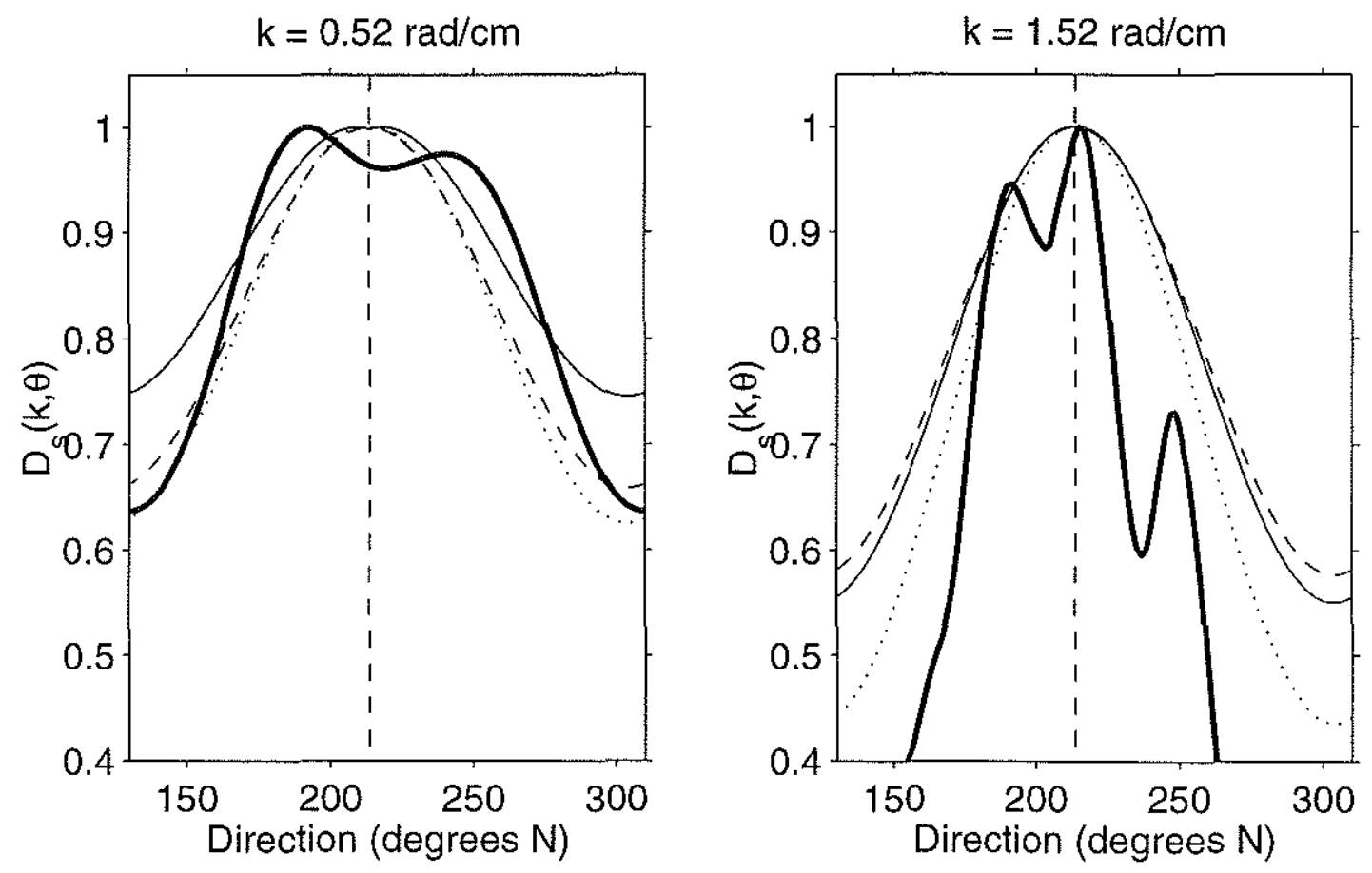

Figure 5-5: Comparison of the Normalized Directional Spreading Models with Data Collected at the Buzzards Bay Tower (10281415). The thick solid line in each plot is a constant wavenumber slice (a circular ring) of the two-dimensional wavenumber frequency spectrum measured by the circular wire wave gage (CWWG). The CWWG measurements are averaged over 20 minutes. The wavenumber above each plot is the wavenumber at which the slice was taken. The thin lines represent the model functions: - - - = Elfouhaily et al., $\cdots=$ Romeiser et al., and - = Caudal et al. The Apel spreading model is not included because it is effectively isotropic at these wavenumbers when symmetrized using equation 5.7 . The plots are angularly centered near the maximum experimental data peak, but the model directional slices are centered on the mean wind direction which is shown as a vertical dashed line at $213^{\circ}$. All the models and the CWWG data are normalized as described in equation 5.8 

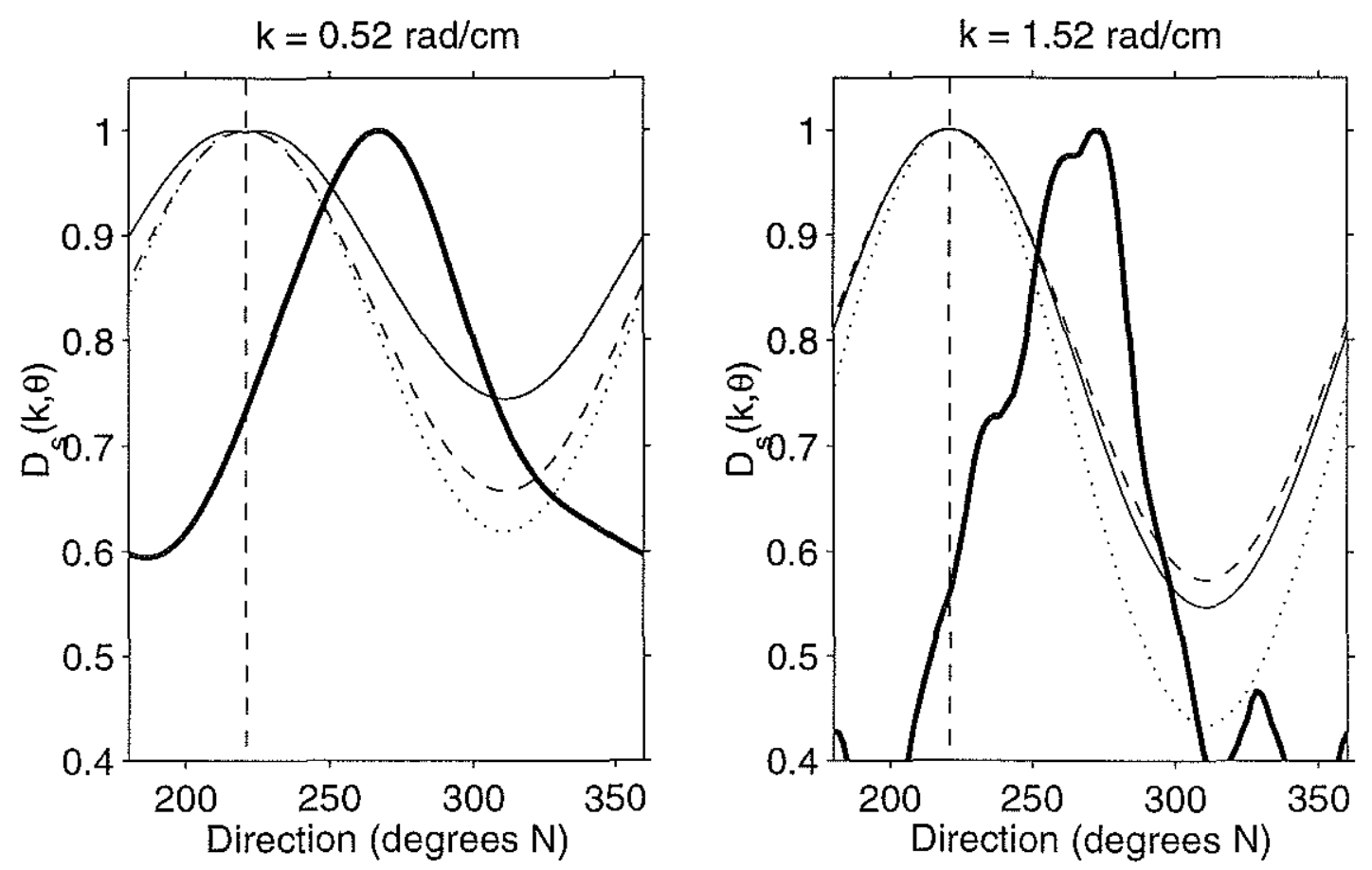

Figure 5-6: Comparison of the Normalized Directional Spreading Models with Data Collected at the Buzzards Bay Tower (10281445). The thick solid line in each plot is a constant wavenumber slice (a circular ring) of the two-dimensional wavenumber frequency spectrum measured by the circular wire wave gage (CWWG). The CWWG measurements are averaged over 20 minutes. The wavenumber above each plot is the wavenumber at which the slice was taken. The thin lines represent the model functions: - - = Elfouhaily et al., $\cdots=$ Romeiser et al., and - = Caudal et al. The Apel spreading model is not included because it is effectively isotropic at these wavenumbers when symmetrized using equation 5.7 . The plots are angularly centered near the maximum experimental data peak, but the model directional slices are centered on the mean wind direction which is shown as a vertical dashed line at $221^{\circ}$. All the models and the CWWG data are normalized as described in equation 5.8 

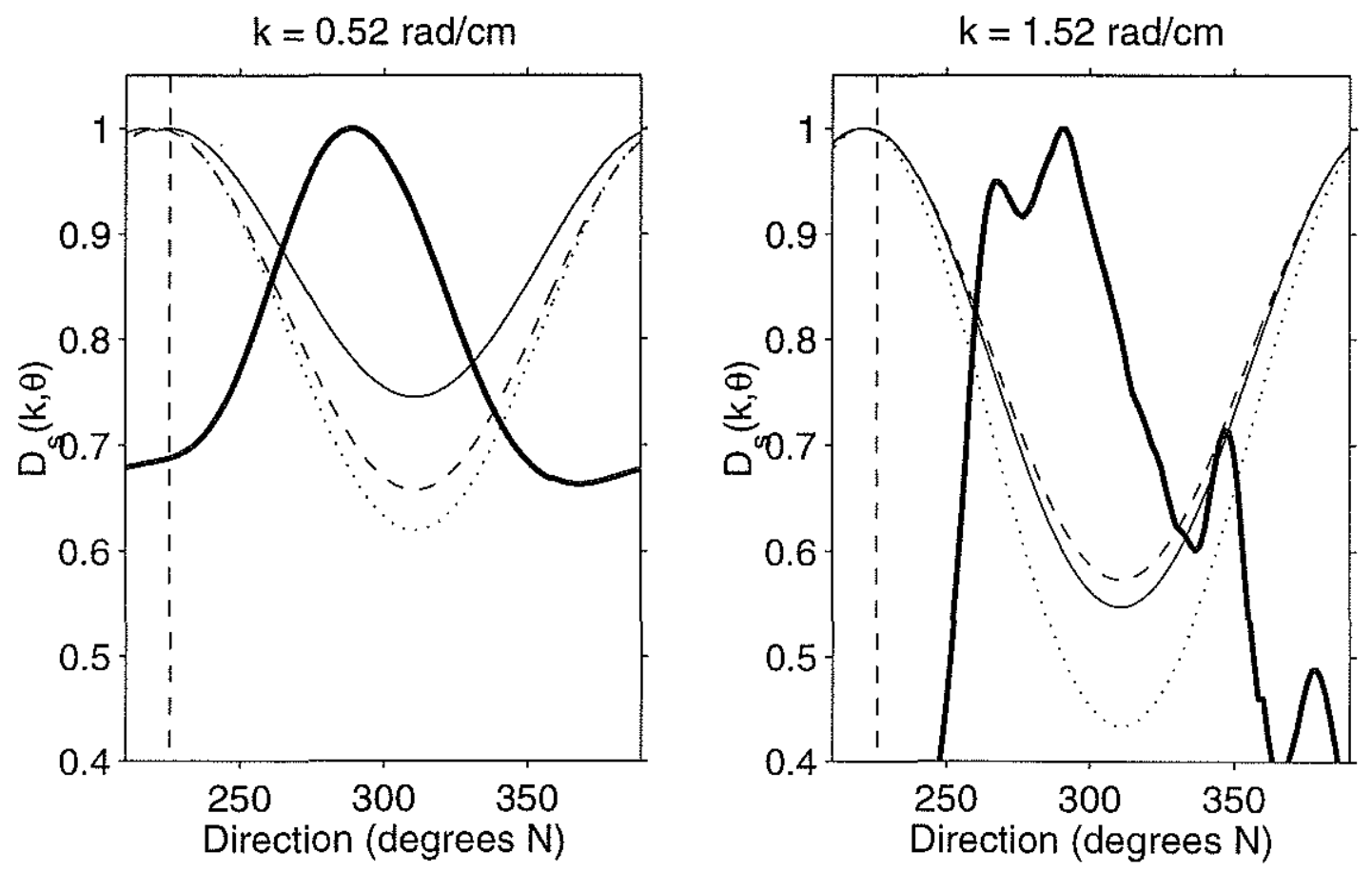

Figure 5-7: Comparison of the Normalized Directional Spreading Models with Data Collected at the Buzzards Bay Tower (10281515). The thick solid line in each plot is a constant wavenumber slice (a circular ring) of the two-dimensional wavenumber frequency spectrum measured by the circular wire wave gage (CWWG). The CWWG measurements are averaged over 20 minutes. The wavenumber above each plot is the wavenumber at which the slice was taken. The thin lines represent the model functions: - - = Elfouhaily et al., $\cdots=$ Romeiser et al., and - = Caudal et al. The Apel spreading model is not included because it is effectively isotropic at these wavenumbers when symmetrized using equation 5.7 . The plots are angularly centered near the maximum experimental data peak, but the model directional slices are centered on the mean wind direction which is shown as a vertical dashed line at $226^{\circ}$. All the models and the CWWG data are normalized as described in equation 5.8 
is presented here to consider possible explanations for the shift in energy direction. There are a number of possible explanations for this energy shift: one, the compass data was not incorporated properly, and two, the meteorological conditions at the location of the buoy shifted over time and were not represented well by measurements made at the Buzzards Bay Tower. With respect to the first, the compass data collected on the buoy was used to reference the local measurements, which shifted as the buoy floated in the waves, to a geographic coordinate system. It was further verified that this referencing was done properly, and the actual orientation of the buoy over the course of the experiment was assessed and is shown in Figure 5-8. These plots show that while the buoy did rotate over the course of the experiment, it did not progressively rotate further and further in one direction which could have affected the continual shift of the peak energy location through possible errors in the processing. With respect to the second possible explanation, it is curious that the first data set is fairly well aligned with the wind measurements made at the tower, but that as the buoy moved further from the tower, the following two data sets show a systematic rotation of the peak energy. The winds further from the tower may actually have shifted, but it is difficult to believe that within three nautical miles of the tower they would have shifted by up to $90^{\circ}$. Another possible explanation was mentioned earlier as a possible explanation for the unexpectedly low wavenumber slope in the wavenumber slice plots - surface currents. These currents could have affected the directional properties of the short waves, but it is difficult to explain the long term shifting unless the buoy was caught in some kind of quasistable eddy of sorts. Unfortunately, there is no way to know for sure as no surface wind or surface current measurements were collected at or near the buoy. Given that the CWWG instrument and processing errors which could have caused these spurious results have been rigorously checked, the precise reason for this behavior is currently unknown.

Figures 5 5, 5-6, and 5-7 illustrate the variability in the peak location of the CWWG data well, but it is difficult to determine if the widths of the measured spectra and the models are comparable, so a calculation of spectral width as a function of wavenumber was generated. A mean square angular width was defined as

$$
W i d t h(k)=\left[\frac{\int\left(\theta-\theta_{C G}\right)^{2} \Phi(k, \theta) d \theta}{\int \Phi(k, \theta) d \theta}\right]^{1 / 2}
$$




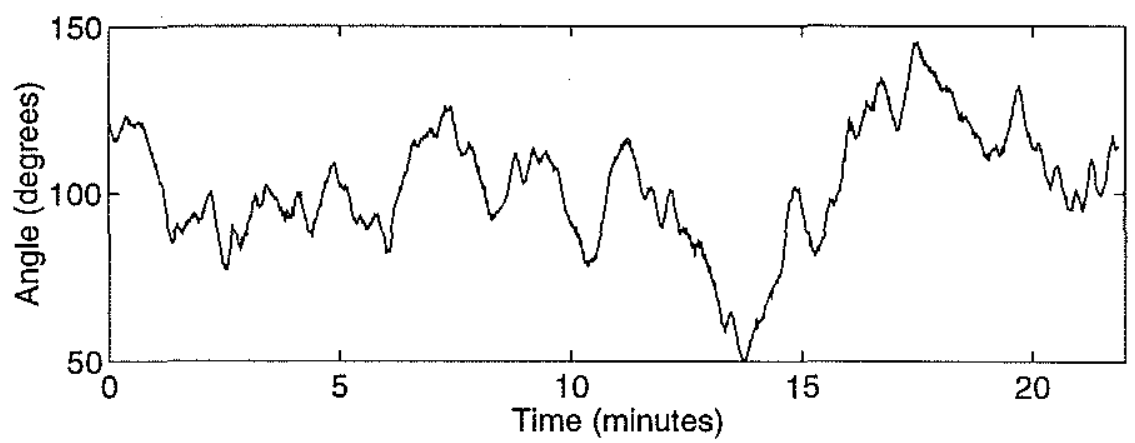

(a)

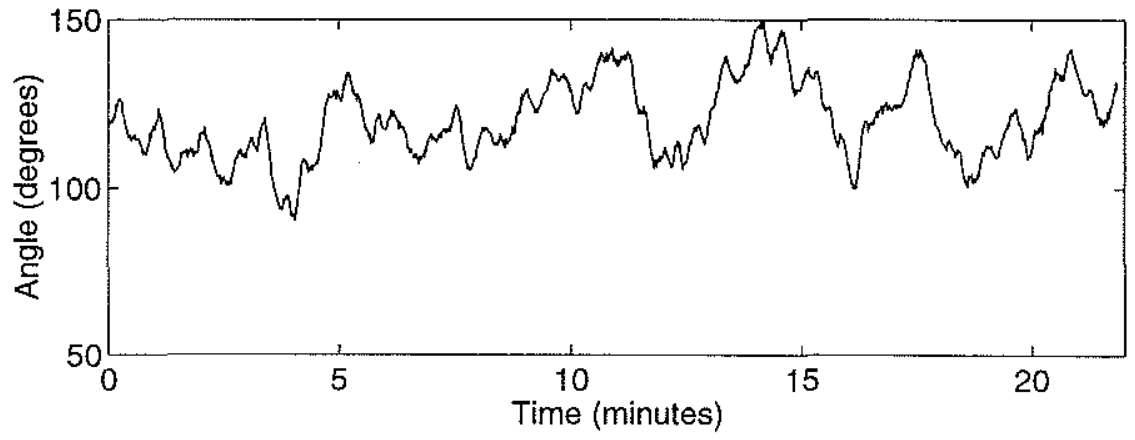

(b)

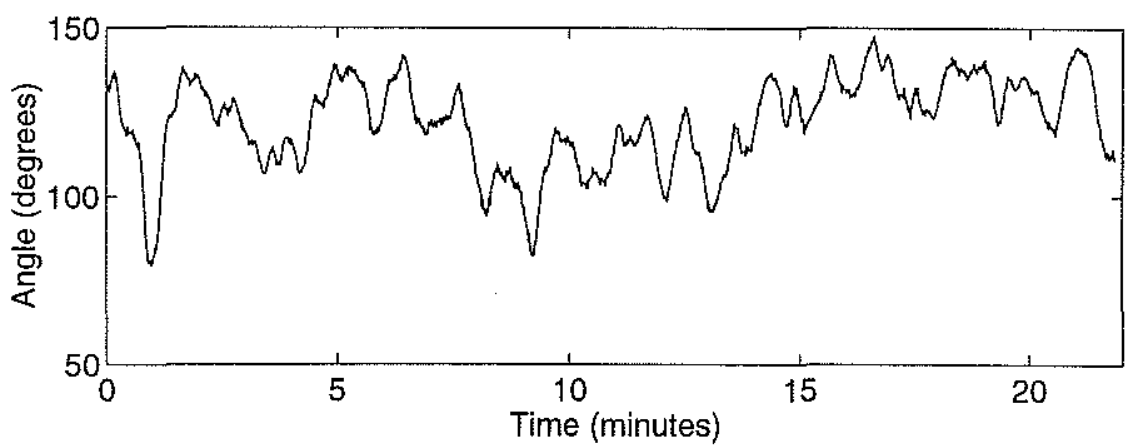

(c)

Figure 5-8: Buoy Orientation. (a) is from data set 10281415, (b) is from data set 10281445, and (c) is from data set 10281515. The angles given are the direction that the compass was facing, but the compass was not aligned with the wind vane on the buoy, so these angles do not correspond to the wind directions presented elsewhere in this thesis. 


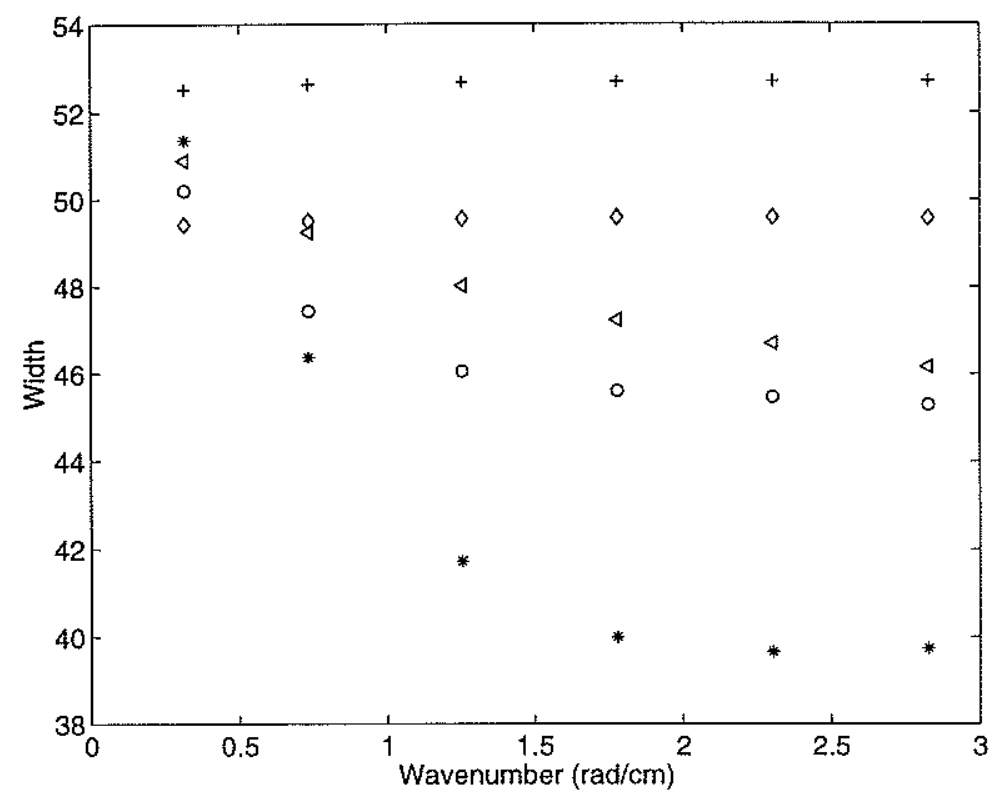

Figure 5-9: Comparison of the Widths of the Directional Spreading Models with Data Collected at the Buzzards Bay Tower (10281415). * = CWWG data, $+=$ Banner model, $\circ=$ Romeiser model, $\triangleleft=$ Caudal model, $\diamond=$ Elfouhaily model The widths are shown in degrees. 20 minutes of data were used to generate these width measurements.

where

$$
\theta_{C G}=\frac{\int \theta \Phi(k, \theta) d \theta}{\int \Phi(k, \theta) d \theta}
$$

and $\Phi(k, \theta)$ is the two-dimensional wavenumber spectrum.

Figure 5-9 shows a calculation of the widths of the CWWG data and the models for data set 10281415. This Figure clearly illustrates that using this measure of width, the CWWG data is narrower than the models predict except at the lowest wavenumber bin. Specifically, the Romeiser and Caudal models propose a slight narrowing of the spectrum as a function of wavenumber, while the Banner and Elfouhaily models do not. The circular wire wave gage data, thus, supports an even greater narrowing of the spectrum than the Romeiser and Caudal models.

Figures 5-5, 5-6, and 5-7 show some abberations in the measured spectra at the higher wavenumbers (they are not very smooth), though. To verify that the width measurements 
have converged and would not simply get wider if more data were averaged, a measure of width as a function of averaging time was generated and is shown in Figure 5-10. An issue that is raised by the plots in Figure 5-10 is the significant (up to $50^{\circ}$ ) variability of the width measurements for short averaging times. Inspection of the two-dimensional wavenumber spectra for these short time period averages indicates that the large widths are not generally indicative of actually broad spectra, but rather are primarily generated by multi peaked spectra which have energy distributed in two distinct regions. It is possible that a different measure of width, say a $3-\mathrm{dB}$ estimator may show a different kind of variability, but some method would still need to be developed to deal with the multi peaked distribution.

\subsection{Electromagnetic Scattering Models}

The scatterometer data collected at the tower offers a slightly different opportunity to see how well the collected data agrees with models which use the surface wave height spectra as an input parameter. The data collected by the X-band scatterometer at the tower exhibits similar azimuthal distribution as other scatterometer data sets available. Figure 511 shows the Buzzards Bay Tower scatterometer azimuthal measurements on a decibel scale $\left[\mathrm{dB}=10 * \log _{10}\right.$ (power)] for the 10281315 data set. The upwind-to-crosswind ratio is approximately 6 for the HH polarization and between 5 and 6 for the VV polarization. Similar ratios exist for the other two scatterometer data sets at the tower. These upwindto-crosswind ratios are nearly identical to those found by Masuko et al. [44] for similar meteorological conditions and incidence angles. Additionally, Feindt et al. [20] include a plot (their Figure 16) of upwind-to-downwind ratios as a function of wavenumber which accurately predicts the ratios measured by the scatterometer at the tower, however they show a large scatter in the data. Interestingly, even accounting for the scatter in the data, the Feindt plot shows an increasing upwind-to-crosswind $\sigma_{o}$ ratio as a function of Bragg wavenumber, indicating a narrowing trend in the scatterometer azimuthal data at higher scatterometer frequencies.

A detailed description of the form of the electromagnetic scattering model relevant to the experimental measurements presented herein was given in Chapter 2. A rather generic form 


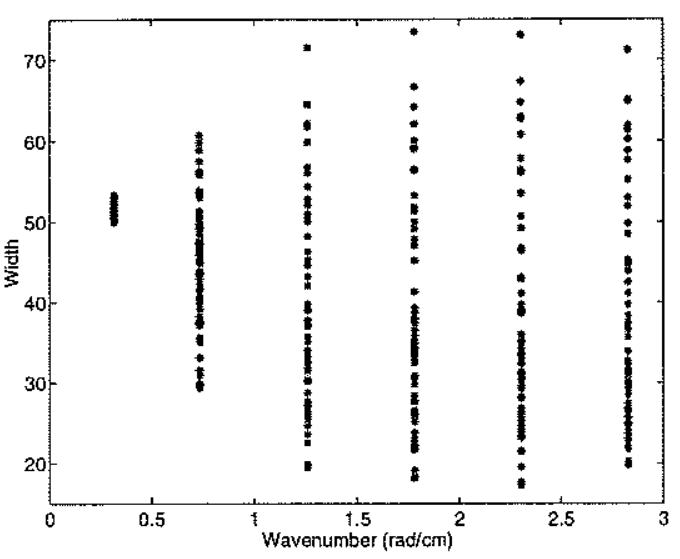

(a) 1 minute averages

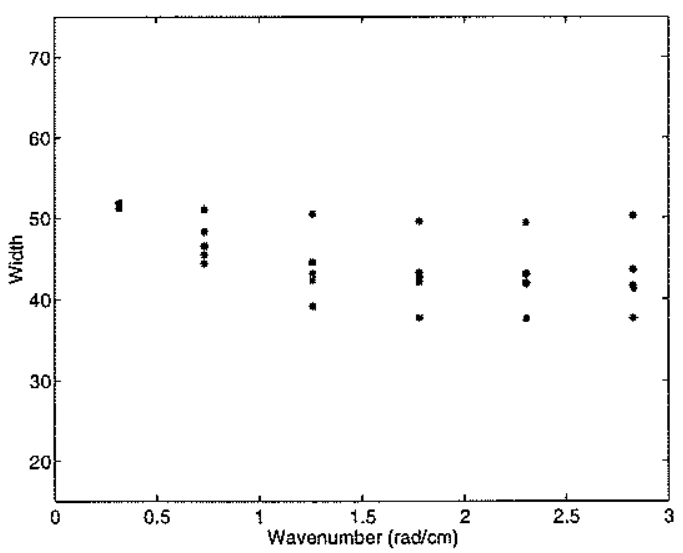

(c) 10 minute averages

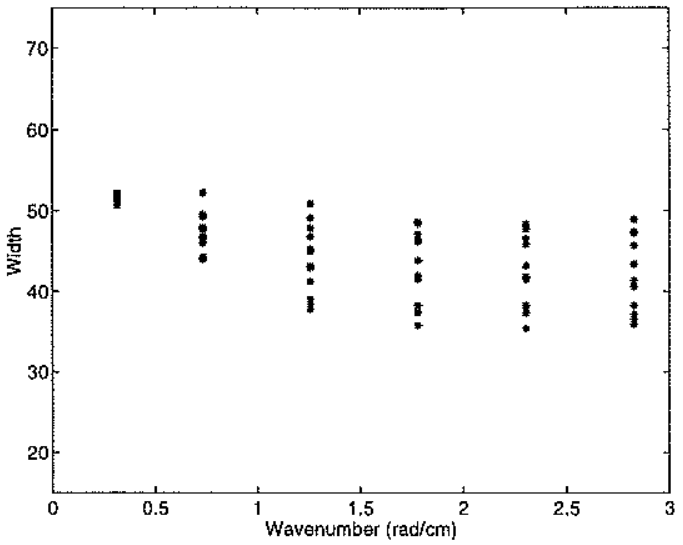

(b) 5 minute averages

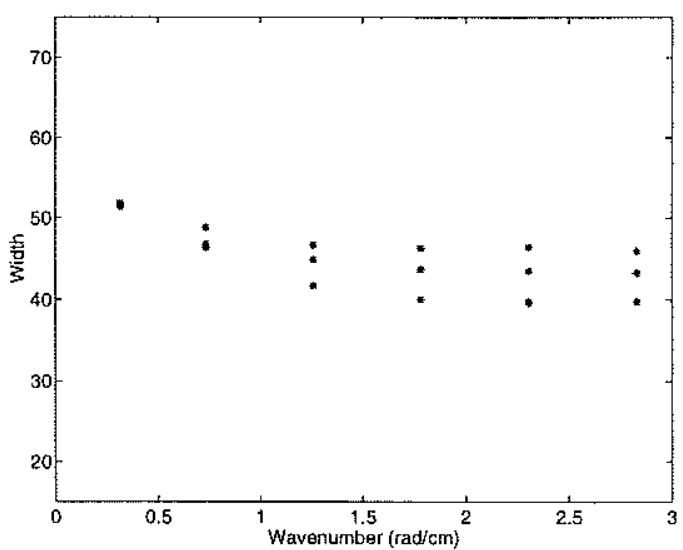

(d) 20 minute averages

Figure 5-10: Convergence of Circular Wire Wave Gage Width Measurements. The width values are calculated using all three spectral data sets (10281415, 10281445, and 10281515). 


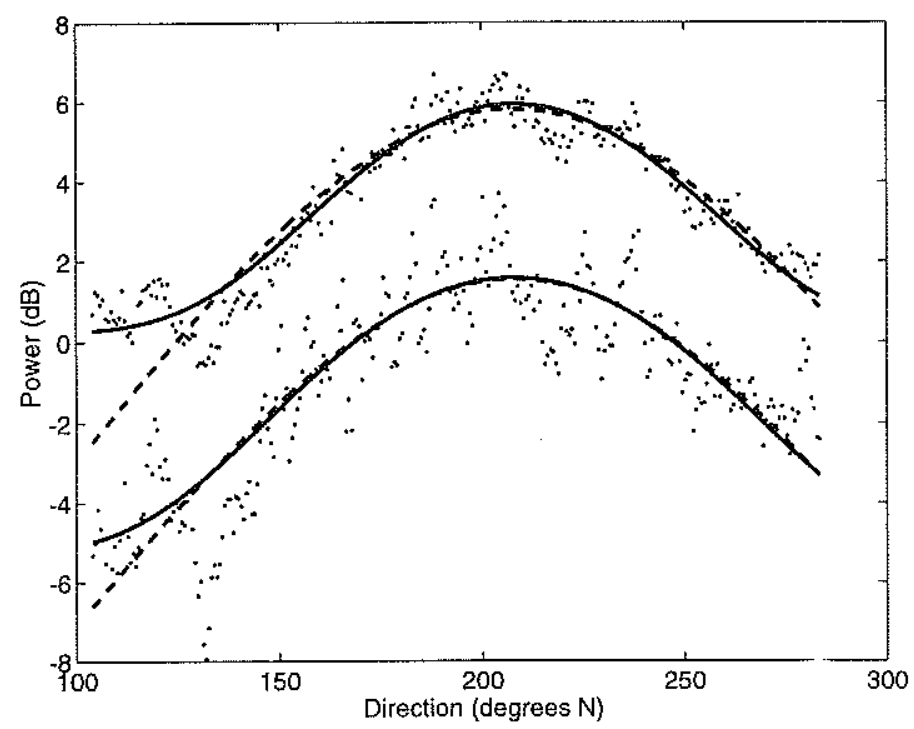

Figure 5-11: Directional X-Band Scatterometer Return from Data Set 10281315. This plot is a replica of Figure 4-17 except that it is shown on a decibel scale. The upper curve and data points are VV polarization and the lower fitted curves and data points are for $\mathrm{HH}$ polarization. The power has units of $\mathrm{I}^{2}+\mathrm{Q}^{2}$ where $\mathrm{I}$ and $\mathrm{Q}$ are measured in volts. The solid line represents a best fit of the data to the function $\cos ^{2 s}\left(\frac{\theta-\theta_{\max }}{2}\right)$, while the dashed line represents a best fit of the data to the function $\cosh ^{-2}\left(\beta\left(\theta-\theta_{\text {max }}\right)\right)$. The $s$ and $\beta$ values for the HH and VV polarizations are given in the Figure 4-17 caption. The data points shown are 85 point averages. 
of this model for linear wave theory which includes the now well established Bragg scattering foundation along with a tilt modulation factor is repeated here with the normalized radar backscatter cross section (NRCS) being given as

$$
\sigma_{0}(\phi, \theta)=8 \pi k_{0}^{4} \int G\left(\phi, \gamma_{L x}, \gamma_{L y}\right) \Gamma\left(\phi^{\prime}, \theta\right) P\left(\gamma_{L x}, \gamma_{L y}\right) d \gamma_{L x} d \gamma_{L y}
$$

where $\phi$ is the incidence angle, $\theta$ is the azimuthal look direction, $k_{0}$ is the electromagnetic wavenumber, $G\left(\phi, \gamma_{L x}, \gamma_{L y}\right)$ is a non-dimensional polarization dependent geometric reflection coefficient, $\Gamma\left(\phi^{\prime}, \theta\right)$ is the folded surface displacement wavenumber spectrum evaluated at the Bragg wavelength, and $P\left(\gamma_{L x}, \gamma_{L y}\right)$ is the probability density of the long wave slopes, $\gamma_{L x}$ and $\gamma_{L y}$. The only two azimuthal dependencies given in this equation are within the folded wavenumber spectrum and the long wave probability distribution.

Using Lyzenga's [41] simplification of Plant's [50] Gaussian approximation of the slope probability distribution detailed in Chapter 2, it was concluded that a skewed probability distribution does have the ability to impact the angular dependence of the NRCS. Using extrapolated data from the CWWG two-dimensional wavenumber spectrum for the shape and magnitude of the short wave input to the scattering model, and using the long wave measurements from the capacitive wire wave gage to estimate the long wave slopes, the output of the modeled scatterometer return with the tilting is narrower than the simple Bragg scattering model return without tilting effects, but the effect is minimal.

Based on the simplified scattering model in equation 5.12 then, the primary cause of the breadth of the scattered return is the width of the wave height spectrum. This is supported by the Buzzards Bay Tower data as shown in Figures 5-12 and 5-13. Both figures show width calculations for both the scatterometer data, the corresponding CWWG data, and the previously described models. As can be seen, the width of the scatterometer data is on the same scale as the widths measured by the CWWG data, but it is still narrower than current spectral models predict. It can be concluded, therefore, that the circular wire wave gage data shows support for the current scattering theories based primarily on Bragg scattering. 


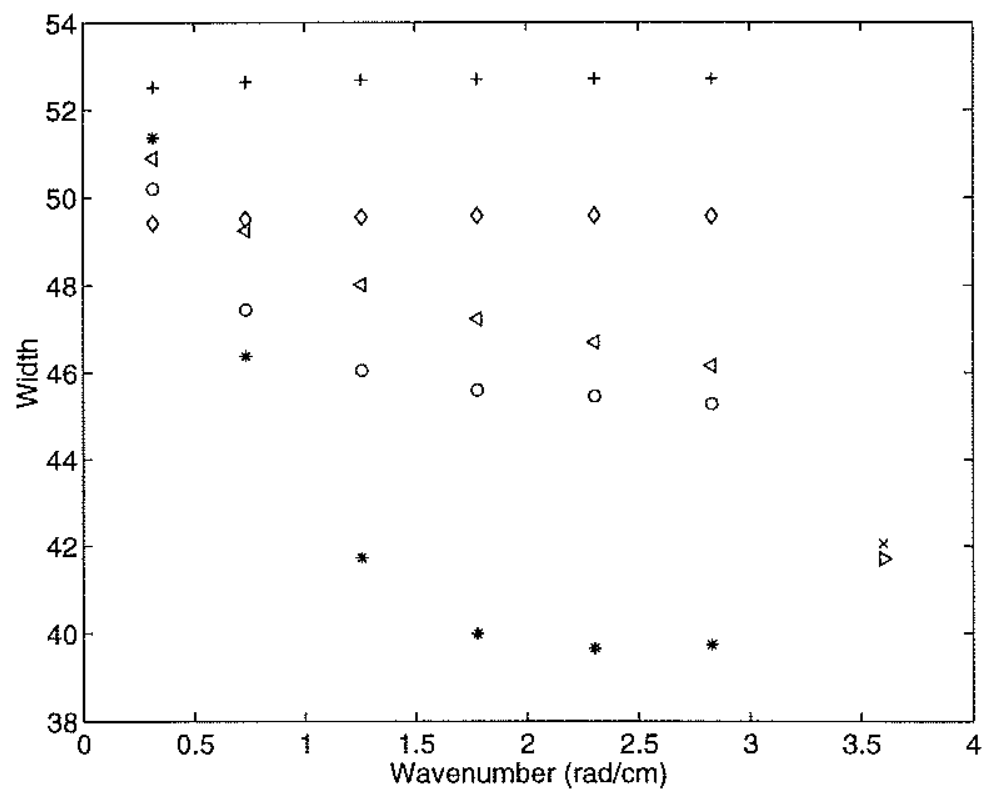

Figure 5-12: Comparison of the Widths Between Spreading Models, the Circular Wire Wave Gage, and the X-Band Scatterometer (10281415(CWWG) and 10281315(scat)). * = CWWG data, $+=$ Banner model, $\circ=$ Romeiser model, $\triangleleft=$ Caudal model, $\diamond=$ Elfouhaily model, $\triangleright=\mathrm{HH}$ scatterometer return width, and $x=\mathrm{VV}$ scatterometer return width. The scatterometer widths are plotted at their approximate Bragg wavenumber. The widths are shown in degrees. 


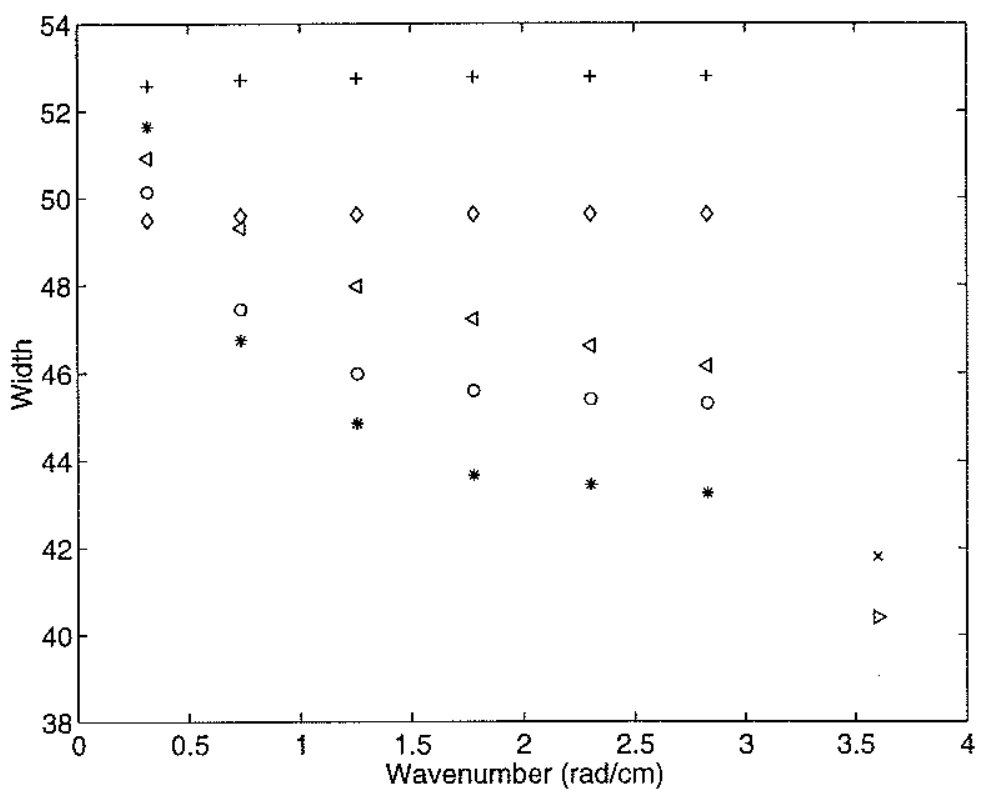

Figure 5-13: Comparison of the Widths Between Spreading Models, the Circular Wire Wave Gage, and the X-Band Scatterometer (10281445(CWWG) and 10281344(scat))). * = CWWG data, $+=$ Banner model, $\circ=$ Romeiser model, $\triangleleft=$ Caudal model, $\diamond=$ Elfouhaily model, $\triangleright=\mathrm{HH}$ scatterometer return width, and $x=$ VV scatterometer return width. The scatterometer widths are plotted at their approximate Bragg wavenumber. The widths are shown in degrees. 


\subsection{Conclusions}

The one- and two-dimensional wavenumber spectral data presented in this and the previous chapter agree with only some of the current model predictions. Specifically, the measured one-dimensional frequency spectra show a slope which increases from $\omega^{-4}$ to near $\omega^{-5}$ at the higher wavenumbers. This agrees with the DHH spectral model at the low frequency end, but behaves much more like earlier models predicting the steeper slope of -5 . However, experimental data in the literature at these frequencies is not consistent and it is believed that the slope of the frequency spectrum lies somewhere between -4 and -5 depending on environmental conditions. Meanwhile, the omnidirectional wavenumber spectrum plots show the data supporting the -2.5 to -3 slopes which are typical in three of the models shown. But in the wavenumber spectral slice plots the CWWG data shows a gradually decreasing slope which is probably associated with physical processes, such as surface currents, which are not incorporated into the models presented here. The wavenumber spectral slice models, thus, predict a slope of near -4 , but the CWWG data never gets below about -3. The cause for the differences between the measured and the modeled frequency and wavenumber slopes are not well understood, but it is believed that both the orbital velocities of the long waves and possible strong surface currents may be somewhat responsible. There is not enough evidence within these data sets to argue this conclusively, however, and further investigation of the surface currents at the Buzzards Bay Tower may shed some light on these discrepancies.

The measured width of the spectra show a narrowing as a function of wavenumber which is supported, to a slightly lesser degree, by only two of the models presented herein. The other models discussed do not support any narrowing as a function of wavenumber. Additionally, the X-band scatterometer data measured at the tower compare very well to other available experimental scatterometer data sets. It was also shown that a simplified composite surface model based on Bragg scattering could reproduce the width of the normalized radar cross-section as measured by the X-band scatterometer at the Buzzards Bay Tower, when given as input an interpolated form of the two-dimensional wave height spectrum.

The only other in situ data avaliable at these wavenumbers was published by Hwang et al. in 1996 [31] and Hara et al. in 1998 [24]. Both Hwang and Hara used scanning laser 
slope gages. These instruments measured slope instead of wave height, and both were able to calculate what is known as a saturation spectrum. The saturation spectrum is generally defined as $k^{4} * S(k)$ where $S(k)$ is the omnidirectional wave height spectrum, or as $k^{2} * S^{s l}(k)$ where $S^{s l}(k)$ is the omnidirectional wave slope spectrum. This spectrum is often used instead of the wave height spectrum for comparative purposes because if it is assumed that there is a saturation limit in the slope of the energy distribution of $k^{4}$ as described in Chapter 2, then the saturation spectrum should have zero slope. The saturation spectra from these two papers at wind friction velocities similar to those experienced during the Buzzards Bay Tower experiment are about two times smaller than the CWWG saturation spectra, but they show similar wavenumber slopes. Field data collected by these authors show large scatter in magnitude of the spectrum, however, and it is not believed that the Buzzards Bay Tower omnidirectional spectrum data constitute any significant divergence from the previous experimental data, especially since the other surface conditions for these experiments are not well matched to those experienced at the Buzzards Bay Tower. Given that these data sets were collected in differing ocean conditions it isn't appropriate, unfortunately, to compare the directional distribution of energy from them with the CWWG data.

Incidentally, a concern about the interpretation of the data with respect to the current models exists because of the shifting of the peak energy direction away from the mean wind direction. As mentioned previously, this may be due to some currently unknown error in the instrument, a true shift in the mean wind direction near the buoy, or a significant surface current effect, but there is nothing to either support or refute any one of these explanations, given that there were no local wind or surface current measurements made at the buoy. The shift may also indicate that at these short wavelengths there is the possibility of significant amounts of energy propagating in directions other than the mean wind direction, but this is hard to explain over the course of a 20 minute average. It is important to note that circular wire wave gage measures the short wave distribution over a patch of the sea surface which is small with respect to the long waves. The resulting averaged spectra represent an estimate of the variable energy distribution over a very small spatial domain. The statistical variability of this process at these scales is not well understood, and without additional and 
more lengthy studies it is difficult to draw any firm conclusions about why the averaged energy distribution is not well aligned with wind measurements in the synoptic area.

While the data presented in this thesis is not comprehensive enough to make conclusive recommendations for slight modification to some of the current models to account for the small variations which are shown in both magnitude of the spectrum and its width, it does indicate that further studies need to be conducted. Specifically, further experimental data with both radars and in situ instruments capable of measuring the two-dimensional wavenumber spectrum over a significant amount of time should be performed. Only through more thorough investigation will the discrepancies between the models and data of this nature finally be resolved. 


\section{Chapter 6}

\section{Summary}

Short waves on the ocean surface play an important role is surface electromagnetic (e.m.) scattering. Electromagnetic scattering theory is a well developed field, but the short wavelength portion of the surface wave field has only recently been experimentally explored, and a single, consistent model of the wave height spectrum has yet to be developed. A new instrument was developed to measure the height of waves with $2-30 \mathrm{~cm}$ wavelengths at an array of locations which can be post-processed to generate an estimate of the surface wave height spectrum. This instrument was deployed in an experiment which gathered not only in situ measurements of the two-dimensional wave height spectrum, but also coincident scatterometer measurements. This thesis describes the development of this instrument and the comparison of the data which was collected during this experiment with current spectral and electromagnetic surface theory models.

This work began with a description of surface wave theory including a basic description of surface wave propagation and current theories specifying the two-dimensional energy distribution out to the gravity-capillary region of the spectrum. A presentation of electromagnetic scattering theory was then given. A brief historical outline of its development was given as well as the mathematical basis to understand the current models. This chapter ended with an account of other instruments available which measure surface wave spectra.

Chapter 3 introduced the circular wire wave gage. It talked about the buoy structure and housings, the layout, modification, and purpose of the analog electronics, the data acquisition hardware, the basic operation of the instrument, as well as the post-deployment 
data processing necessary to generate the wave height spectrum estimate.

The last two chapters described the Buzzards Bay Tower experiment and the comparison if the resulting data with current models. The instrumentation usage and layout description was followed with plots and images of all of the meteorological, buoy, and scatterometer data collected. The circular wire wave gage data processing was also compared with that of another directional spectrum measuring instrument to validate its methods. The wave height spectrum as measured by the circular wire wave gage was then compared to the models presented in Chapter 2. It was found that while the omnidirectional spectrum measurements were similar in slope but slightly larger in magnitude than the models, the spectral slice from the in situ data in the dominant wave direction showed variability both in slope and magnitude. This was evident also in the two-dimensional wavenumber spectrum estimates generated from the CWWG data. It was shown that the azimuthal spreading of these spectral plots was only slightly underestimated by the current spectral models. The data collected at the Buzzards Bay Tower was also useful for comparison with current scatterometer models. Specifically, it was shown that the directional spreading measured by the circular wire wave gage when extrapolated out to the Bragg wavelength of the Xband scatterometer used, was very near the corresponding azimuthal width measured by the scatterometer. Given that the azimuthal dependence in most current linear theory composite surface theory scattering models is entirely derived from the spreading of the surface wave height spectrum, this data presents new evidence that these models are very close to accurately modeling the Bragg spreading mechanisms.

\subsection{Future Work}

The circular wire wave gage has provided new information about short wave distribution on a platform that is easy to deploy. There are certainly improvements which would enhance its performance and usefulness for short wave studies, however. These include adding a 'motion-pack' system capable of monitoring pitch, heave, and roll, allowing long wave information to be ascertained which could potentially provide the ability to subsample the data at just the wave peaks or troughs allowing actual in situ measurements of the differences 
between the short surface wave energy distribution over the long wave profile. A wind sensor mounted on the buoy would also help with temporal correlation of the wave data with the wind data. Additionally, surface currents and long wave orbital velocities may affect the short wave spectra as was suggested in Chapter 5, so future deployments incorporating colocated surface current measurements would be very informative. Finally, while twodimensional wavenumber spectra provide very useful data about energy distribution on the ocean surface, this information could be significantly enhanced with the addition of information in the third dimension: time. It is possible to obtain the three-dimensional wave height spectrum from the circular wire wave gage data up to a frequency of nearly 50 $\mathrm{Hz}$ given that each scan is collected at $100 \mathrm{~Hz}$, but analysis and data processing algorithms have not yet been developed for this purpose.

While the Buzzards Bay Tower experiment generated new information about the validity of current wavenumber spectrum and electromagnetic scattering models, it did not provide enough information to generate model corrections or to suggest exactly which processes caused the differences between the experimental data and the models. Additional studies are needed to look at the directional characteristics of short sea waves over a series of varying meteorological conditions in conjunction with azimuthal scattering, specifically because the variability in directional properties of short sea waves may be significant and is highly dependent on wind and wave conditions. 


\section{Bibliography}

[1] John R. Apel. An improved model of the ocean surface wave vector spectrum and its effects of radar backscatter. Journal of Geophysical Research, 99(C8):16,269-16,291, 1994.

[2] M. L. Banner, Ian S. F. Jones, and J. C. Trinder. Wavenumber spectra of short gravity waves. Journal of Marine Research, 198:321-344, 1989.

[3] Michael L. Banner. Equilibrium spectra of wind waves. Journal of Physical Oceanography, 20:966-984, 1990.

[4] F. G. Bass, I. M. Fuks, A. I. Kalmykov, I. E. Ostrovsky, and A. D. Rosenberg. Very high frequency radiowave scattering by a disturbed sea surface, Part I: Scattering from a slightly disturbed boundary. IEEE Transactions on Antennas and Propagation, AP16(5):554-559, 1968.

[5] F. G. Bass, I. M. Fuks, A. I. Kalmykov, I. E. Ostrovsky, and A. D. Rosenberg. Very high frequency radiowave scattering by a disturbed sea surface, Part II: Scattering from an actual sea surface. IEEE Transactions on Antennas and Propagation, AP16(5):560-568, 1968.

[6] J. A. Battjes, T. J. Zitman, and L. H. Holthuijsen. A reanalysis of the spectra observed in JONSWAP. Journal of Physical Oceanography, 17:1288-1295, 1987.

[7] A. W. Bjerkaas and F. W. Riedel. Proposed model for the elevation spectrum of a windroughened sea surface. Technical Report TG 1328, JHU/APL Technical Memorandum, December 1979. 
[8] Erik J. Bock and Tetsu Hara. Optical measurements of capillary-gravity wave spectra using a scanning laser slope gauge. Journal of Atmospheric and Oceanic Technology, $12(2): 395-403,1995$.

[9] G. Caudal and D. Hauser. Directional spreading function of the sea wave spectrum at short scale, inferred from multifrequency radar observations. Journal of Geophysical Research, 101(C7):16,601-16,613, 1996.

[10] Rick Chapman and F. M. Monaldo. A novel wave height sensor. Journal of Atmospheric and Oceanic Technology, 12(1):190-196, 1995.

[11] H. Charnock. Wind stress on a water surface. Quarterly Journal of the Royal Meteorological Society, 81:639-640, 1955.

[12] David Joel Chen. Designing wave-measuring instruments. Master's thesis, Massachusetts Institute of Technology, 1994.

[13] John P. Comstock, editor. Principles of Naval Architecture. The Society of Naval Architects and Marine Engineers, New York, 1967.

[14] C. Cox and W. Munk. Statistics of the sea surface derived from sun glitter. Journal of Marine Research, 13:198-227, 1954.

[15] Russ E. Davis and Lloyd A. Regier. Methods for estimating directional wave spectra from multi-element arrays. Journal of Marine Research, 35(3):453-477, 1977.

[16] M. A. Donelan and Willard J. Pierson. The sampling variability of estimates of spectra of wind-generated waves. Journal of Geophysical Research, 88(C7):4381-4392, 1983.

[17] Mark A. Donelan, J. Hamilton, and W. H. Hui. Directional spectra of wind-generated waves. Philosophical Transacations of the Royal Society of London, A 315:509-562, 1985.

[18] Mark A. Donelan and Willard J. Pierson. Radar scattering and equilibrium ranges in wind-generated waves with application to scatterometry. Journal of Geophysical Research, 92(C5):4971-5029, 1987. 
[19] T. Elfouhaily, B. Chapron, and K. Katsaros. A unified directional spectrum for long and short wind-driven waves. Journal of Geophysical Research, 102(C7):15781-15796, 1997.

[20] Folkart Feindt, Volkmar Wismann, Werner Alpers, and William C. Keller. Airborne measurements of the ocean radar cross section at $5.3 \mathrm{GHz}$ as a function of wind speed. Radio Science, 21(5):845-856, 1986.

[21] R. M. Goldstein, F. Li, J. Smith, R. Pinkel, and T. P. Barnett. Remote sensing of ocean waves: The Surface Wave Process Program experiment. Journal of Geophysical Research, 99(C4):7945-7950, 1994.

[22] A. Guissard. Directional spectrum of the sea surface and wind scatterometry. International Journal of Remote Sensing, 14(8):1615-1633, 1993.

[23] Kurt A. Hanson. Open-ocean observations of the modulation of gravity-capillary waves by long gravity waves. Master's thesis, University of Rhode Island, Graduate School of Oceanography, 1996.

[24] Tetsu Hara, Erik J. Bock, James B. Edson, and Wade R. McGillis. Observation of short wind waves in coastal waters. Journal of Physical Oceanography, 28:1425-1438, 1998.

[25] Tetsu Hara and William J. Plant. Hydrodynamic modulation of short wind-wave spectra by long waves and its measurement using microwave backscatter. Journal of Geophysical Research, 99(C5):9767-9784, 1994.

[26] D. E. Hasselmann, M. Dunckel, and J. A. Ewing. Directional wave spectra observed during JONSWAP 1973. Journal of Physical Oceanography, 10:1264-1280, 1980.

[27] K. Hasselmann, D. B. Ross, P. Muller, and W. Sell. A parametric wave prediction model. Journal of Physical Oceanography, 6:200-228, 1976.

[28] L. H. Holthuijsen. Observations of the directional distribution of ocean-wave energy in fetch-limited conditions. Journal of Physical Oceanography, 13:191-207, 1983. 
[29] B. A. Hughes, H. L. Grant, and R. W. Chappell. A fast response surface-wave slope meter and measured wind-wave moments. Deep-Sea Research, 24:1211-1223, 1977.

[30] Paul A. Hwang. A study of the wavenumber spectra of short water waves in the ocean. Part II: Spectral model and mean square slope. Journal of Atmospheric and Oceanic Technology, 14:1174-1186, 1997.

[31] Paul A. Hwang, Serhad Atakturk, Mark A. Sletten, and Dennis B. Trizna. A study of the wavenumber spectra of short water waves in the ocean. Journal of Physical Oceanography, 26(7):1266-1285, 1996.

[32] Paul A. Hwang, Dennis B. Trizna, and Jin Wu. Spatial measurements of short wind waves using a scanning laser slope sensor. Dynamics of the Ocean and Atmosphere, 20:1-23, 1993.

[33] Bernd Jähne. Energy balance in small-scale waves - An experimental approach using optical slope measuring technique and image processing. In G. J. Komen and W. A. Oost, editors, Radar Scattering from Modulated Wind Waves, pages 105-120. Kluwer Academic Publishers, Norwell, MA, 1989.

[34] Bernd Jähne and Klaus S. Riemer. Two-dimensional wave number spectra of smallscale water surface waves. Journal of Geophysical Research, 95(C7):11,531-11,546, 1990.

[35] J. R. Jensen. Correcting phase circularity errors in radar data. Technical Report STR-89-16, Johns Hopkins University, Applied Physics Laboratory, April 1989.

[36] A. T. Jessup, W. K. Melville, and W. C. Keller. Breaking waves affecting microwave backscatter, 1. Detection and verification. Journal of Geophysical Research, 96(C11):20,547-20,559, 1991.

[37] Blair Kinsman. Wind Waves: Their Generation and Propagation on the Ocean Surface. Prentice-Hall, Inc., New Jersey, 1965. 
[38] P. A. Lange, B. Jähne, J. Tschiersch, and I. Ilmberger. Comparison between and amplitude-measuring wire and a slope-measuring laser water wave gauge. Review of Scientific Instruments, 53(5):651-655, 1982.

[39] P. H. Y. Lee, J. D. Barter, K. L. Beach, C. L. Hindman, B. M. Lake, H. Rungaldier, J. C Shelton, A. B. Williams, R. Yee, and H. C Yuen. X-band microwave backscattering from ocean waves. Journal of Geophysical Research, 100(C2):2591-2611, 1995.

[40] M. S. Longuet-Higgins, D. E. Cartwright, and N. D. Smith. Observations of the directional spectrum of sea waves using the motions of a floating buoy. In Ocean Wave Spectra, pages 111-136. Prentice Hall, New Jersey, 1963.

[41] David R. Lyzenga. Interaction of short surface and electromagnetic waves with ocean fronts. Journal of Geophysical Research, 96(C6):10765-10772, 1991.

[42] David R. Lyzenga, A. L. Maffett, and R. A. Shuchman. The contribution of wedge scattering to the radar cross section of the ocean surface. IEEE Transactions on Geoscience and Electronics, GE-21:502-505, 1993.

[43] Stanislaw R. Massel. Ocean Surface Waves: Their Physics and Prediction. World Scientific Publishing Co., New Jersey, 1996.

[44] Harunobu Masuko, Ken'ichi Okamoto, Masanobu Shimada, and Shuntaro Niwa. Measurement of microwave backscattering signatures of the ocean surface using $\mathrm{X}$ band and $\mathrm{Ku}$ band airborne scatterometers. Journal of Geophysical Research, 91(C11):13,065$13,083,1986$.

[45] L. F. McGoldrick. A sensitive linear capacitance-to-voltage converter, with applications to surface wave measurements. Review of Scientific Instruments, 42(3):359-361, 1971.

[46] Hisashi Mitsuyasu. Measurement of the high-frequency spectrum of ocean surface waves. Journal of Physical Oceanography, 7:882-891, 1977.

[47] Marc Perlin, Huanjay Lin, and Chao-Lung Ting. On parasitic capillary waves generated by steep gravity waves: An experimental investigation with spatial and temporal measurements. Journal of Fluid Mechanics, 255:597-620, 1993. 
[48] O. M. Phillips. The equilibrium range in the spectrum of wind-generated waves. Journal of Marine Research, 4:426-434, 1958.

[49] Willard J. Pierson and Lionel Moskowitz. A proposed spectral form for fully developed wind seas based on the similarity theory of S. A. Kitaigorodskii. Journal of Geophysical Research, 69(24):5181-5190, 1964.

[50] William J. Plant. Two-scale model of short wind-generated waves and scatterometry. Journal of Geophysical Research, 91(C9):10,735-10,749, 1986.

[51] William J. Plant. Bragg scattering of electromagnetic waves from the air/sea interface. In G. L. Geernaert and W. J. Plant, editors, Surface Waves and Fluxes, volume II, pages 41-108. Kluwer Academic Publishers, the Netherlands, 1990.

[52] William J. Plant and William C. Keller. Evidence of Bragg scattering in microwave Doppler spectra of sea return. Journal of Geophysical Research, 95(C9):16,299-16,310, 1990.

[53] William J. Plant, William C. Keller, Vahid Hesany, Tetsu Hara, Erik Bock, and Mark A. Donelan. Bound waves and Bragg scattering in a wind-wave tank. Journal of Geophysical Research, 104(C2):3243-3263, 1999.

[54] S. O. Rice. Reflections of electromagnetic waves from slightly rough surfaces. Communications in Pure Applied Mathematics, 4:351-378, 1951.

[55] Roland Romeiser and Werner Alpers. An improved composite surface model for the radar backscattering cross section of the ocean surface 1 . Theory of the model and optimization/validation by scatterometer data. Journal of Geophysical Research, 102(C11):25,237-25,250, 1997.

[56] Roland Romeiser and Werner Alpers. An improved composite surface model for the radar backscattering cross section of the ocean surface 2. Model response to surface roughness variations and the radar imaging of underwater bottom topography. Journal of Geophysical Research, 102(C11):25,251-25,267, 1997. 
[57] J. A. Saxton and J. A. Lane. Electrical properties of sea water. Wireless Engineering, $29: 169-275,1952$.

[58] Anke Schmidt and Mingquan Bao. The modulation of radar backscatter by long ocean waves: A quadratically nonlinear process? Journal of Geophysical Research, 103(C3):5551-5562, 1998.

[59] Dale L. Schuler. Remote sensing of directional gravity wave spectra and surface currents using a microwave dual-frequency radar. Radio Science, 13(2):321-331, 1978.

[60] William M. Siebert. Circuits, Signals, and Systems. The MIT Press, Cambridge, Massachusetts, 1986.

[61] Nicole Marie Suoja. Development of a directional wave gage for short sea waves. Master's thesis, Massachusetts Institute of Technology, 1996.

[62] Y. Toba. Local balance in the air-sea boundary process III: On the spectrum of wind waves. Journal of the Oceanographic Society of Japan, 29:209-225, 1973.

[63] G. Tober, R. C. Anderson, and O. H. Shemdin. Laser instrument for detecting water ripple slopes. Applied Optics, 12(3):788-794, 1973.

[64] Dennis B. Trizna. A model for Brewster angle damping and multipath effects on the microwave radar sea echo at low grazing angles. IEEE Transactions on Geoscience and Remote Sensing, 35(5):1232-1244, 1997.

[65] Fawwaz T. Ulaby, Richard K. Moore, and Adrian K. Fung. Micorwave Remote Sensing, Active and Passive, volume II. Addison-Wesley Publishing Company, Reading, MA, 1982.

[66] B. Mete Uz. Gravity-capillary waves and their influence on air-sea interaction under non-stationary conditions. PhD dissertation, University of Rhode Island, Graduate School of Oceanography, 1999.

[67] G. R. Valenzuela. Theories for the interaction of electromagnetic and ocean waves - A review. Boundary-Layer Meteorology, 13:61-85, 1978. 
[68] E. J. Walsh, D. W. Hancock III, D. E. Hines, R. N. Swift, and J. F. Scott. Directional wave spectra measured with the surface contour radar. Journal of Physical Oceanography, 15:566-592, 1985.

[69] J. W. Wright. A new model for sea clutter. IEEE Transactions on Antennas and Propagation, AP-16(2):217-223, 1968.

[70] Jin Wu, S. P. Haimbach, and Y.-H. L. Hsu. Scanner for measuring fine sea-surface structures. Review of Scientific Instruments, 52:1246-1251, 1981.

[71] Xin Zhang and Charles S. Cox. Measuring the two-dimensional structure of a wavy water surface optically: A surface gradient detector. Experiments in Fluids, 17:225-237, 1994. 


\section{Appendix A}

\section{Spectral Model References}

Table A.1: Chronological Listing of Spectral Model References (1950's - 1990's).

exp. = experimental, spread. = azimuthal spreading, $\mathrm{spec} . \quad=$ spectrum Most of the articles references here are from one of the following journals: Journal of Geophysical Research, Journal of Physical Oceanography, Journal of Marine Research, or the Journal of Fluid Mechanics. Full reference information for some of these articles can be found in the bibliography.

\begin{tabular}{|l|l|l|}
\hline Author(s) & Development & Output Form \\
\hline Phillips [1958] & dimensional analysis & $\omega \mathrm{spec}$ \\
Cox [1958] & & \\
Pierson \& Moskowitz [1964] & exp. (Moskowitz data) & $\omega$ spec. \\
Ewing [1969] & & \\
Valenzuela et al. [1971] & & \\
Black [1974] & theoretical & $\omega$ spec. \\
Tyler et al. [1974] & exp. (SAR fit) & $2 \mathrm{D} \mathrm{k} \mathrm{spec.}$ \\
Stillwell et al. [1974] & & \\
Mitsuyasu et al. [1975] & exp. (cloverleaf buoy) & 2D $\omega$ \& spread. \\
Kitaigorodskii et al. [1975] & theoretical & $\omega \&$ k spec. \\
\hline
\end{tabular}

continued on next page 
continued from previous page

\begin{tabular}{|c|c|c|}
\hline Author(s) & Development & Output Form \\
\hline Larson \& Wright [1975] & exp. (tank/e.m. backsct.) & \\
\hline Long \& Huang [1976] & & \\
\hline Plant \& Wright [1977] & & \\
\hline $\mathrm{Wu}[1977]$ & & 2D slope spec \\
\hline Regier et al. [1977] & & \\
\hline Mitsuyasu [1977] & & \\
\hline Reece, Jr. [1978] & & \\
\hline Bjerkaas \& Riedel [1979] & theoretical \& experimental & $\omega \& \mathrm{k} \mathrm{spec}$ \\
\hline Lleonart et al. [1980] & & \\
\hline Hasselman et al. [1980] & exp. (JONSWAP/pitch-roll buoy) & 2D $\omega \&$ spread. \\
\hline Kahama [1981] & & \\
\hline Huang et al. [1981] & exp. (JONSWAP) & k spec. \\
\hline Fung \& Lee [1982] & experimental (Radar) & $\omega \& \mathrm{k}$ spec. \\
\hline Kitaigorodskii [1983] & theoretical \& experimental & \\
\hline Holthuijsen [1983] & exp. (N. Sea/stereophotography) & $2 \mathrm{D}$ k \& spread. \\
\hline Tang \& Shemdin [1983] & exp. (MARSEN/laser slope follow) & $\omega$ spec. \\
\hline Komen et al. [1984] & theoretical & 2D $\omega$ spec. \\
\hline Jackson et al. [1985b] & & \\
\hline Hwang [1985] & exp. (Atlantic/laser slope) & $2 \mathrm{D} \mathrm{k}$ spectrum \\
\hline Phillips $[1985]$ & theoretical \& experimental & $2 \mathrm{D} \mathrm{k}$ spectrum \\
\hline Donelan et al. [1985] & exp. (Lake Ontario/wave staff fit) & $\omega$ spec., spread. \\
\hline Haimback \& Wu [1986] & exp. (Lab/scanning slope gage) & prob. dist. spread. \\
\hline Donelan \& Pierson [1987] & exp. & \\
\hline Atakturk et al. [1987] & exp. (Lake WA./wave gage) & $\omega$ spec \\
\hline Shemdin \& Hwang [1988] & exp. (MARSEN \& TOWARD/laser) & slope, $\omega$ spec. \\
\hline Shemdin et al. [1988] & exp. (TOWARD/stereophoto.) & $2 \mathrm{D} \mathrm{k} \mathrm{spec.}$ \\
\hline
\end{tabular}


continued from previous page

\begin{tabular}{|c|c|c|}
\hline Author(s) & Development & Output Form \\
\hline Banner et al. [1989] & exp. (BHP platform/stereophoto) & $2 \mathrm{D} \mathrm{k} \mathrm{spec}$ \\
\hline Jahne [1989] & exp. (Delft tank/opt grad \& sl) & slope $\omega \& \mathrm{k}$ spec. \\
\hline Banner [1990] & exp. (previous data) & 1D spec, spread. \\
\hline Jahne \& Riemer [1990] & exp. (Delft tank/optical grad.) & 2D k slope spec. \\
\hline \multicolumn{3}{|l|}{ Bock \& Hara [1992] } \\
\hline \multicolumn{3}{|l|}{ Klinke \& Jahne [1992] } \\
\hline Jackson et al. [1992] & $\exp$. (radar) & mean sq. slope \\
\hline Apel $[1994]$ & exp. (Donelan, Banner, Jahne) & k spec. \\
\hline Caudal \& Proud'hom [1994] & theoretical dvlpmnt of $\sigma_{\circ}$ & $\mathrm{k}$ spec. anisotropy \\
\hline Young [1994] & theoretical dvlpmnt of array config. & spread. \\
\hline Hara et al. [1994] & exp. comparison btw gage \& radar & $\omega$-k slope spec. \\
\hline Hara \& Plant [1994] & exp. (SAXON \& MARSEN/radar) & $\mathrm{MTF}$ \\
\hline Zhang [1995] & exp. (Scripps tank/optical grad.) & k spec \\
\hline Young et al. [1995] & exp. (Lk. George, AU wave gage) & direc. spread. \\
\hline Liu \& Yan [1995] & theoretical dvlpmnt/ERS-1 comp. & k spec. \& spread. \\
\hline Caudal \& Hauser [1996] & exp. (JONSWAP, Donelan) & direc. spread. \\
\hline \multicolumn{3}{|l|}{ Kitaigorodskii [1996] } \\
\hline Hwang et al. [1996] & exp. (HiRes ARI) & k spec. \\
\hline Elfouhaily et al. [1997] & exp. (JONSWAP, Phillips, Kitai.) & 2-D k spec. \\
\hline Hara et al. [1997] & exp. (Canada flume/laser slope) & $\omega$-k sl spec., spread. \\
\hline Hwang [1997] & exp. (Atlantic/laser slope, radar) & k spec. \\
\hline Romeiser et al. [1997] & exp. (airborne scat.) & k spec., spread. \\
\hline Hanson et al. [1997] & exp. (catamaran/laser slope) & $2 \mathrm{D} \omega$ spec. \\
\hline
\end{tabular}




\section{Appendix B}

\section{Scattering Model References}

Table B.1: Chronological Listing of Scattering Model References (1950's - 1990's). exp. = experimental, spread. = azimuthal spreading, spec. $=$ spectrum, theo. $=$ theoretical Most of the articles references here are from one of the following journals: Journal of Geophysical Research, Journal of Physical Oceanography, Journal of Marine Research, IEEE Transactions on Geoscience and Remote Sensing, or the Journal of Fluid Mechanics. Full reference information for some of these articles can be found in the bibliography.

\begin{tabular}{|l|l|l|}
\hline Author(s) & Development & Frequency(s) \\
\hline Rice [1951] & theoretical (perturbation method) & \\
Wright [1966] & experimental & X-band \\
Barrick et al. [1968] & theoretical (composite surf. theory) & \\
Wright [1968] & theoretical \& exp. (tank) & P,L,C,X-band \\
Bass et al. [1968] & theoretical (perturbation method) & \\
Valenzuela \& Laing [1970] & & \\
Wright \& Keller [1971] & & \\
Long [1974] & & \\
Klein \& Swift [1977] & & \\
\hline
\end{tabular}

continued on next page 
continued from previous page

\begin{tabular}{|c|c|c|}
\hline Author(s) & Development & Frequency(s) \\
\hline Brown [1978] & \multirow{6}{*}{ full theo. development } & \multirow{6}{*}{$400-8910 \mathrm{MHz}$} \\
\hline Valenzuela [1978] & & \\
\hline Jones et al. [1978] & & \\
\hline Alpers \& Hasselmann [1978] & & \\
\hline Alpers et al. [1981] & & \\
\hline Fung \& Lee [1982] & & \\
\hline Plant \& Keller [1983] & \multirow[t]{6}{*}{ exp. (MARSEN), MTF } & \multirow[t]{12}{*}{$\mathrm{X}, \mathrm{L}$-band } \\
\hline Lyzenga et al. [1983] & & \\
\hline Wentz et al. [1984] & & \\
\hline Kwoh \& Lake [1984] & & \\
\hline Keller et al. [1985] & & \\
\hline Hasselmann et al. [1985] & & \\
\hline Durden \& Vesecky [1985] & \multirow[t]{6}{*}{ exp. (RADSCAT \& others) } & \\
\hline Plant $[1986]$ & & \\
\hline Masuko et al. [1986] & & \\
\hline Holliday et al. [1986] & & \\
\hline Fung \& Pan [1987] & & \\
\hline Pan \& Fung [1987] & & \\
\hline Donelan \& Pierson Jr. [1987] & \multirow[t]{4}{*}{ full theo. development/data sigma-o } & \multirow[t]{4}{*}{$\mathrm{L}, \mathrm{C}, \mathrm{X}, \mathrm{Ka}$ band } \\
\hline Phillips [1988] & & \\
\hline Wetzel $[1990]$ & & \\
\hline Brown [1990] & & \\
\hline Plant $[1990]$ & \multirow[t]{2}{*}{ exp. (ocean, lake/Doppler spec.) } & \multirow[t]{2}{*}{$1.5-14.0 \mathrm{GHz}$} \\
\hline Trizna et al. [1991] & & \\
\hline Giovanangeli et al. [1991] & \multirow[t]{2}{*}{ exp. (azimuthal tank studies) } & \multirow[t]{2}{*}{$36 \mathrm{GHz}$} \\
\hline Jessup et al. [1991a] & & \\
\hline
\end{tabular}

continued on next page 


\begin{tabular}{|c|c|c|}
\hline Author(s) & Development & Frequency(s) \\
\hline Jessup et al. [1991b] & & \\
\hline Kasilingam et al. [1992] & & \\
\hline Ebuchi et al. [1993] & $\exp .(a b)$ & \\
\hline Wetzel [1993] & & \\
\hline Trizna et al. [1993] & & \\
\hline Apel [1994] & & $5.5-35 \mathrm{GHz}$ \\
\hline Hara \& Plant [1994] & exp. (SAXON \& MARSEN), MTF & \\
\hline Liu \& Pierson [1994] & & \\
\hline Plant et al. [1994a] & & \\
\hline Lee et al. [1995] & exp. (Scotland/Doppler spec.) & $\mathrm{X}$-band \\
\hline Trizna [1996] & & X-band \\
\hline Trizna [1997] & theoretical (Brewster angle) & $\mathrm{L}, \mathrm{S}, \mathrm{C}, \mathrm{X}, \mathrm{Ku}, \mathrm{Ka}$-band \\
\hline Romeiser \& Alpers [1997] & theoretical & \\
\hline Plant [1997] & exp. (SAXON), Doppler & X-band \\
\hline
\end{tabular}




\section{Appendix $\mathrm{C}$}

\section{Wave Measuring Instrument}

\section{References}

Table C.1: Chronological Listing of Wave Measuring Instrument References (1950's-1990's). exp. = experimental, spread. = azimuthal spreading, spec. = spectrum The Output Form column also indicates whether the work is based primarily on field experiments or whether it is based on laboratory work. Most of the articles references here are from one of the following journals: Journal of Geophysical Research, Journal of Phys ical Oceanography, Journal of Marine Research, or the Journal of Fluid Mechanics. Full reference information for some of these articles can be found in the bibliography.

\begin{tabular}{|l|l|l|}
\hline Author(s) & Instrument Type & Output Form \\
\hline Cox \& Munk [1954] & Aerial Photogrammetry & field, slope stats. \\
Cote et al. [1960] & Aerial Photogrammetry & \\
Barber [1961] & Array Directional Resolution & theoretical development \\
Longuet-Higgins et al. [1963] & Floating Buoy & field \\
Prettyman et al. [1969] & Laser Slope Gage & \\
Stillwell [1969] & Single Image Photography & \\
Wu et al. [1969] & & \\
\hline
\end{tabular}

continued on next page 
continued from previous page

\begin{tabular}{|c|c|c|}
\hline Author(s) & Instrument Type & Output Form \\
\hline Garret [1969] & Capacitive Probe & field, f spec. \\
\hline McGoldrick [1970] & Capacitive Probe & \\
\hline Barrick [1972] & High Frequency (HF) Radar & \\
\hline Tober et al. [1973] & Laser Slope Gage & lab, $\omega$ slope spec. \\
\hline Sturm \& Sorrell [1973] & Laser Slope Gage / 1 pt. & lab \\
\hline Sugimori [1975] & Stereo Photography & \\
\hline Hughes et al. [1977] & Laser Slope Gage & \\
\hline Palm et al. [1977] & Laser Slope Gage & \\
\hline Davis \& Regier, L. [1977] & Wire Array Design (resistive) & field, $\omega$-k spec. \\
\hline Schuler, D. [1978] & Dual Frequency Radar & field \\
\hline Lubard et al. [1980] & Opt. grad. vs. Laser Slope & \\
\hline Wu et al. [1981] & Laser Scanning Slope Gage & \\
\hline Lange et al. [1982] & Ampl. vs. Slope Comparison & lab, $\omega$ slope spec. \\
\hline Tang \& Shemdin [1983] & Optical Slope Gage (on wave follower) & field, f spec. \\
\hline Keller \& Gotwols [1983] & Optical & \\
\hline Holthuijsen [1983] & Stereo Photography & \\
\hline Donelan et al. [1985] & Capacitive Wire Wave Array & lab/field, 2D k spec. \\
\hline Haimbach \& Wu [1985] & Optical Scanner & \\
\hline Hasselman et al. [1985] & Synthetic Aperture Radar & \\
\hline Walsh et al. [1985] & Surface Contour Radar & \\
\hline Ebuchi et al. [1987] & Specular Scattering & lab \\
\hline Shemdin et al. [1988] & Stereophotography & field, $2 \mathrm{D} \mathrm{k}$ spec. \\
\hline Smith \& McCardle [1990] & Capacitive Multiwire Buoy & field, $2 \mathrm{D} \mathrm{k}$ spec. \\
\hline Jahne \& Riemer [1990] & Surface Light Gradient & lab \\
\hline Lyzenga et al. [1991] & 2-ant. SAR & \\
\hline Li et al. [1993] & Laser Slope Gage & lab/field, 2D k spec \\
\hline
\end{tabular}

continued on next page 
continued from previous page

\begin{tabular}{|l|l|l|}
\hline Author(s) & Instrument Type & Output Form \\
\hline Hwang et al. [1993] & Laser Slope Gage & lab, 2D k spec \\
Chapman \& Monaldo [1993] & Capacitive Wire Wave Gage & lab/field \\
Goldstein et al. [1994] & SAR/Acoustic Doppler & field, 2D $\omega$ spec. \\
Zhang \& Cox [1994] & Surface Gradient Detector & lab, 2D k sl. spec. \\
Sletten et al. [1994] & Polarimetric Radar & lab \\
Bock \& Hara [1995] & Laser Slope Gage & lab/field, $\omega$-k spec. \\
\hline
\end{tabular}




\section{Appendix D}

\section{Analog Electronics Interface Board Electrical Diagram}

The diagram shown in Figure D-1 represents the electrical schematic for the circular wire wave gage analog electronics interface board. This board is located in the central analog electronics housing and acts as the interface between the wave wires, the analog electronics, and the data acquisition system located in a separate housing. 


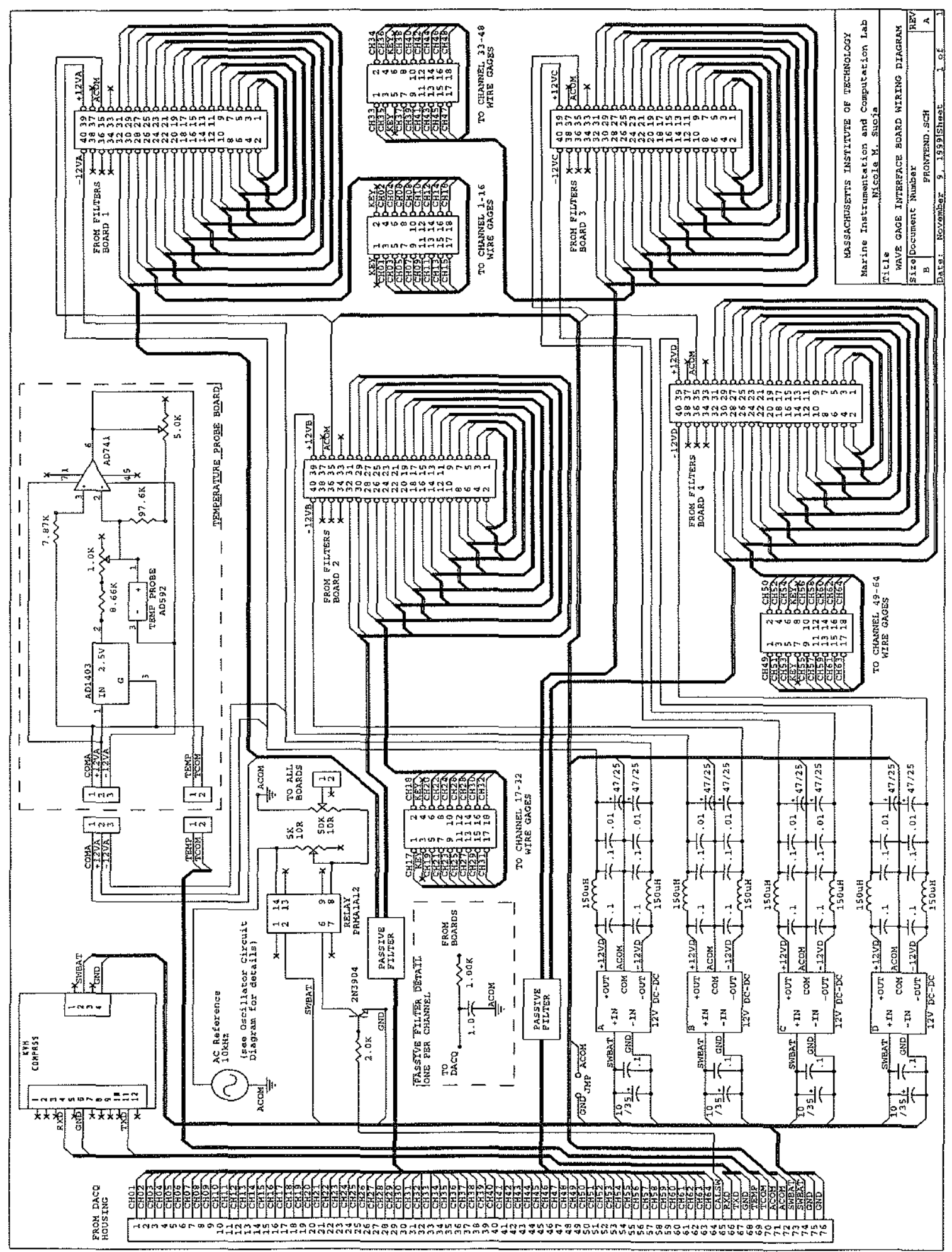

Figure D-1: Analog Electronics Interface Board 


\section{Appendix E}

\section{Oscillator Circuit Electrical}

\section{Diagram}

The diagram shown in Figure E-1 represents the electrical schematic for the circular wire wave gage oscillator. This small board is mounted on the larger wiring interface board in the central analog electronics housing, and its output is the buffered high-frequency carrier signal used by all the channels. This signal is referred to as the $10 \mathrm{kHz}$ sine wave input on the other circular wire wave gage analog circuit diagrams. 


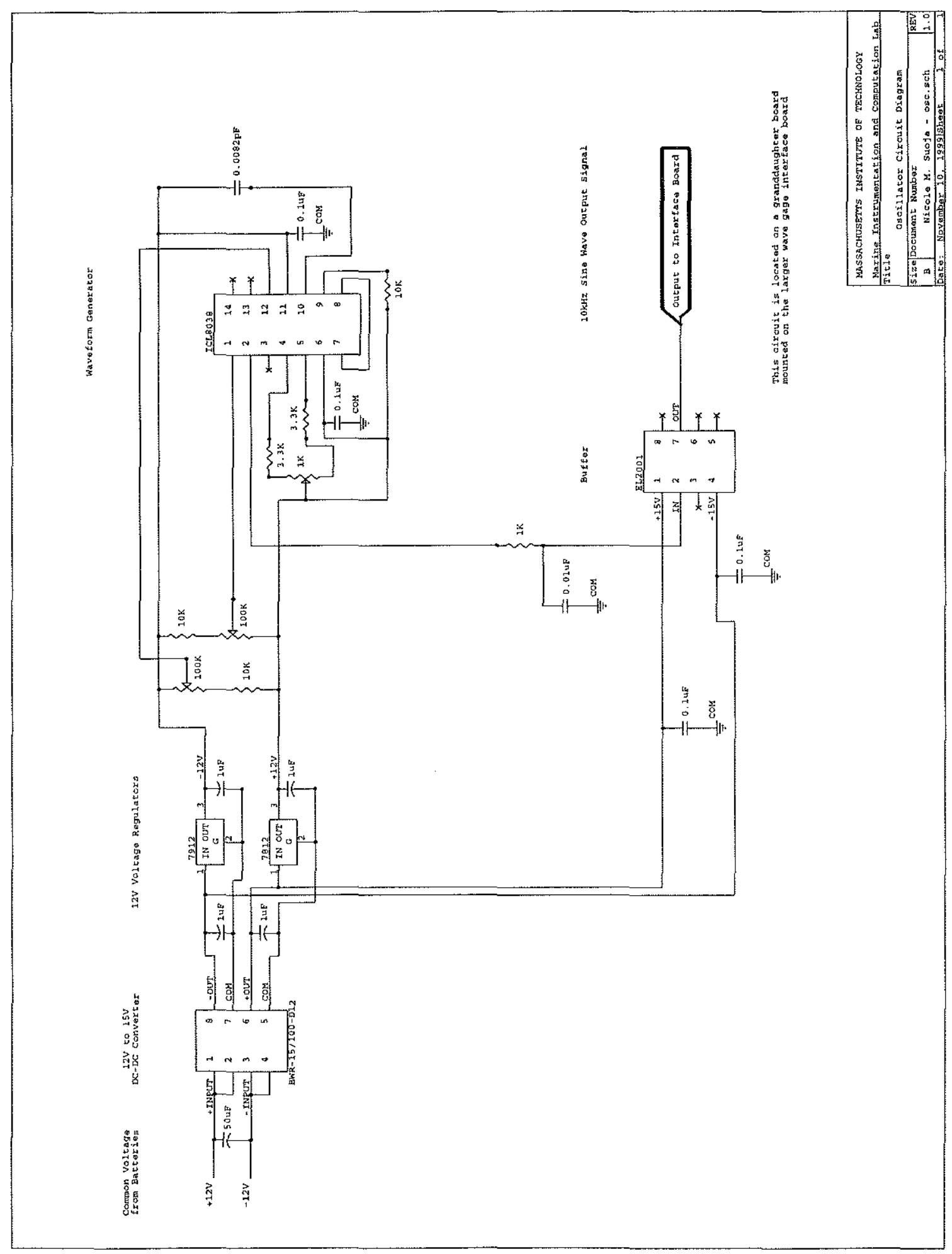

Figure E-1: Oscillator Circuit Diagram 


\section{Appendix $\mathbf{F}$}

\section{Data Acquisition Design Criteria}

The data acquisition system was designed to meet the following criteria

- Sample a set of data channels at $100 \mathrm{~Hz}$.

- Collect (scan) each set of 64 channels as quickly as possible (needs to be collected quickly enough that 'stationarity' of the scan can be assumed)

- Store collected data from a typical day's deployment on board the buoy

- Provide means to download the data from the buoy to another computer without having to disassemble the data acquisition system

- Sample the on-board compass at least once every second

- Sample the temperature probe at least once every 5 to 8 seconds

- Sample the battery voltage regularly and shut off if below a specified cutoff level

- Minimize power consumption so as to minimize the number of batteries needed

- Acquisition system must fit inside an 8-inch diameter aluminium housing

- Provide easy-to-use command line operation of data acquisition program which allows for calibration and for user to define data set length, interval between data sets, and absolute start time 


\section{Appendix G}

\section{Data Acquisition Hardware}

\section{Schematic}

The diagram shown in Figure G-1 represents the general schematic for the circular wire wave gage data acquisition system. The PC104 form-factor motherboard and associated hardware are mounted to vertical struts which are secured to the endcap of the data acquisition aluminum housing. 


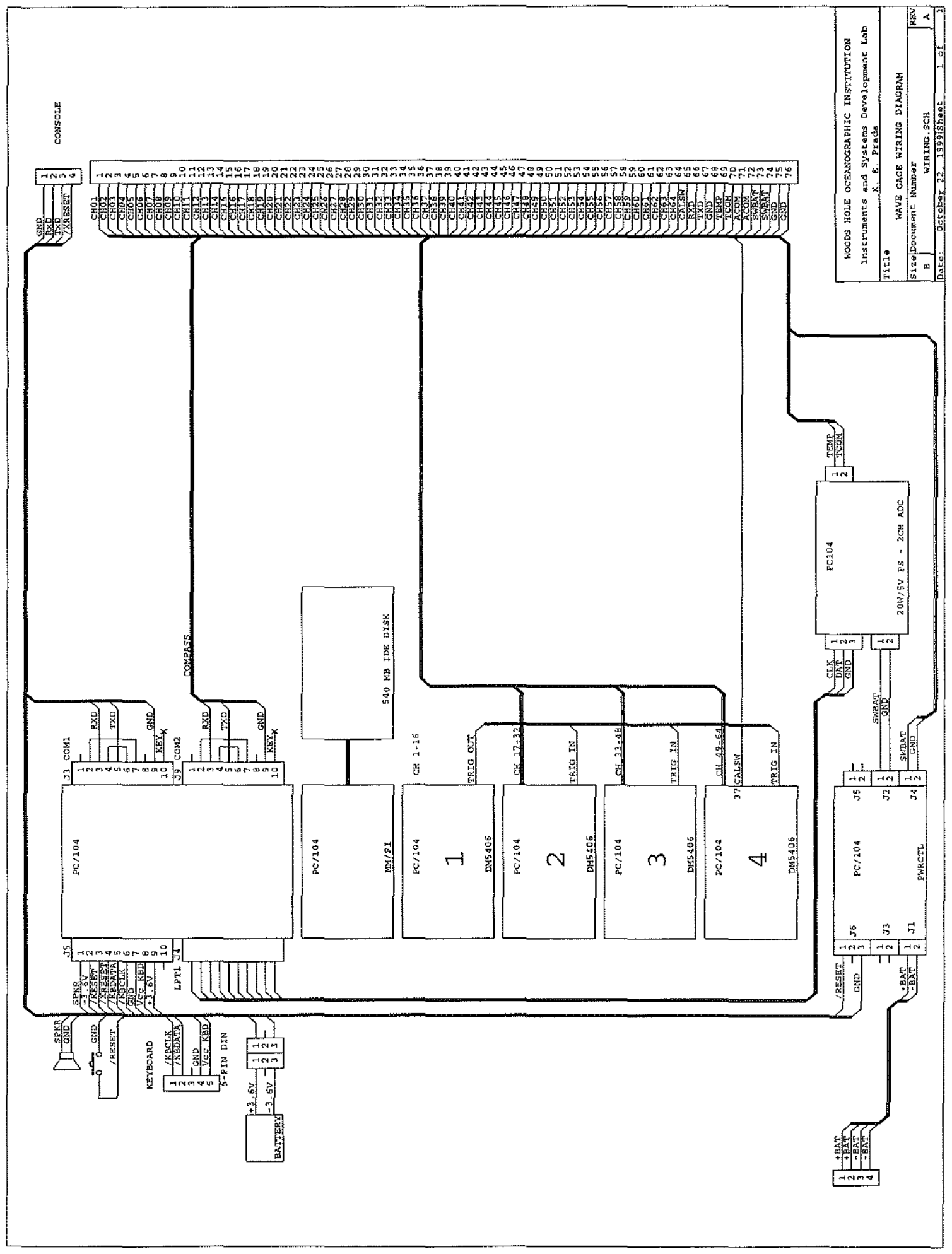

Figure G-1: Data Acquisition Hardware Schematic 


\section{Appendix $\mathbf{H}$}

\section{Data Acquisition Software}

The following two programs control the general data acquisition of the circular wire wave gage. The first program is wave. $c$ which manages the actual analog to digital conversion and data storage. The second program, setup.c, directs the watchdog timer to turn the instrument on and off based on user input.

\section{H.1 Wave.c}

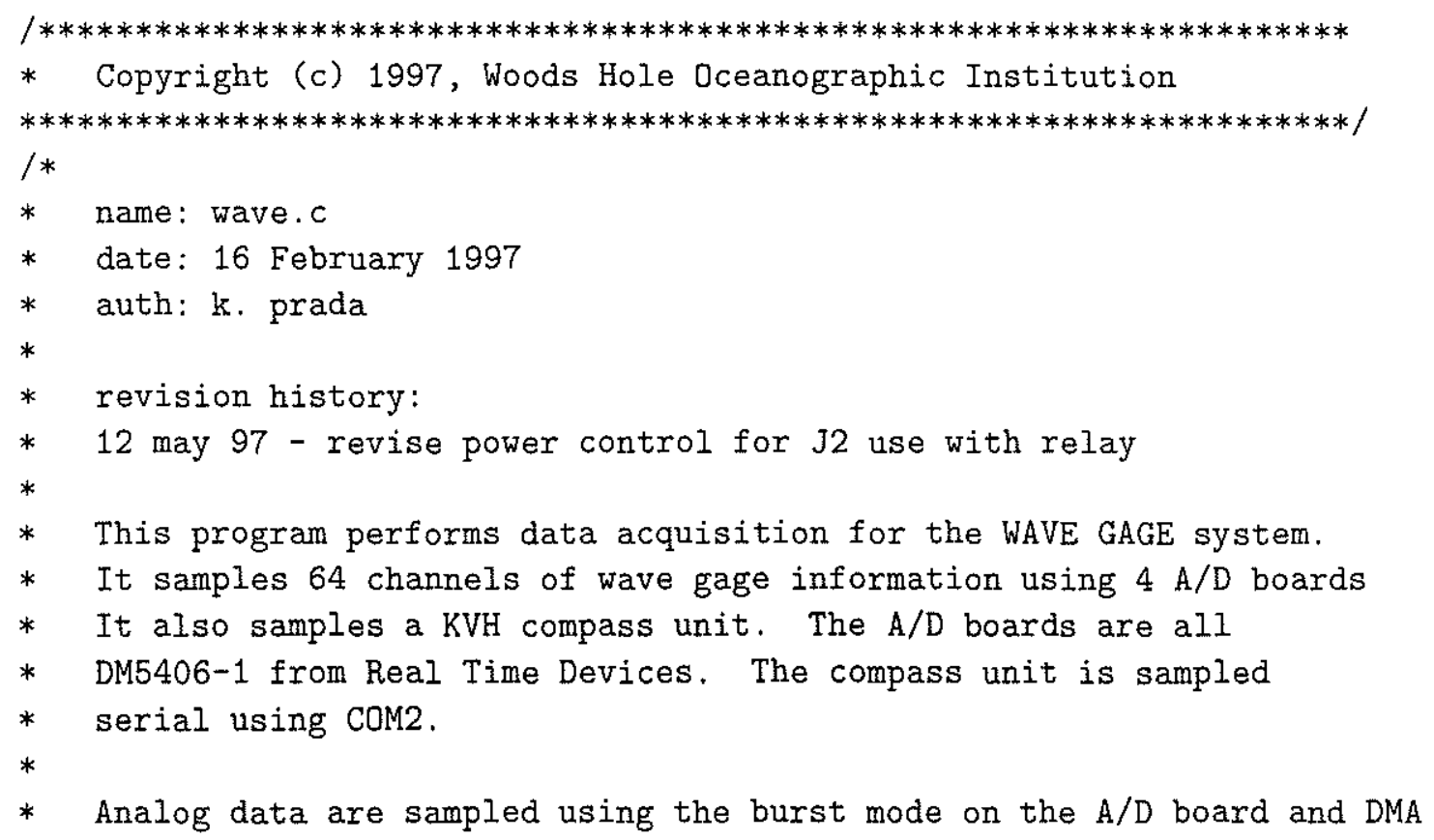




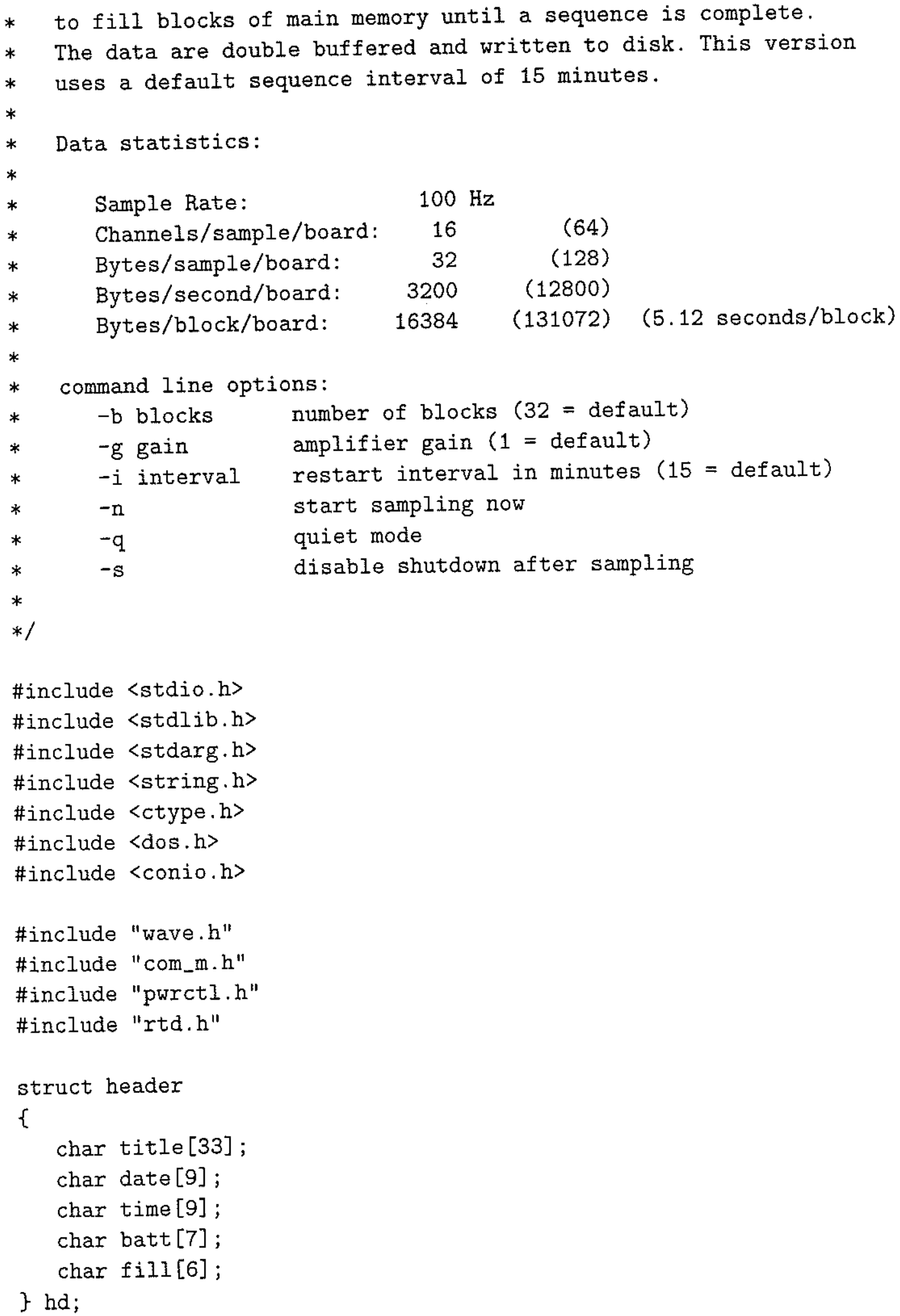




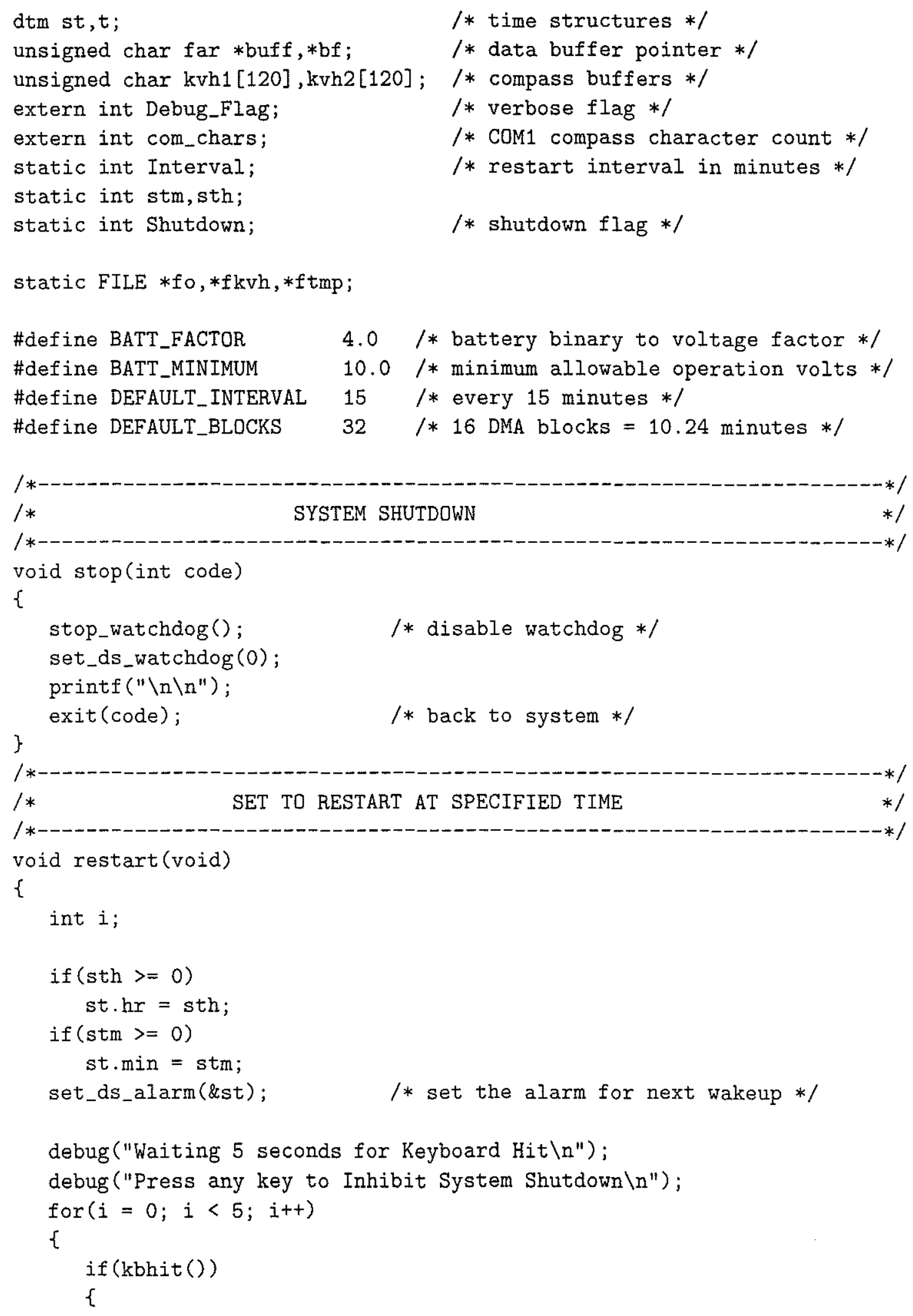




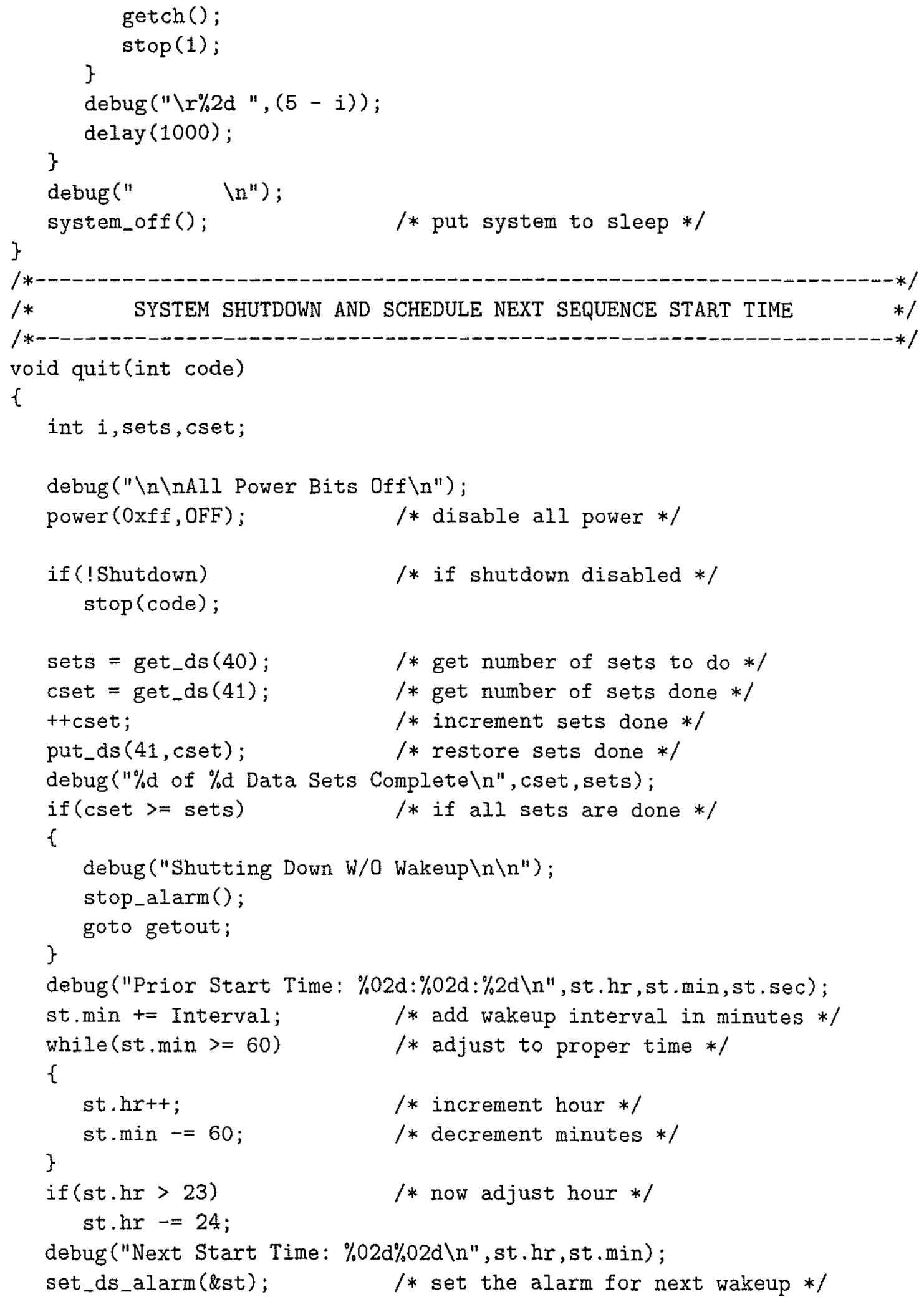




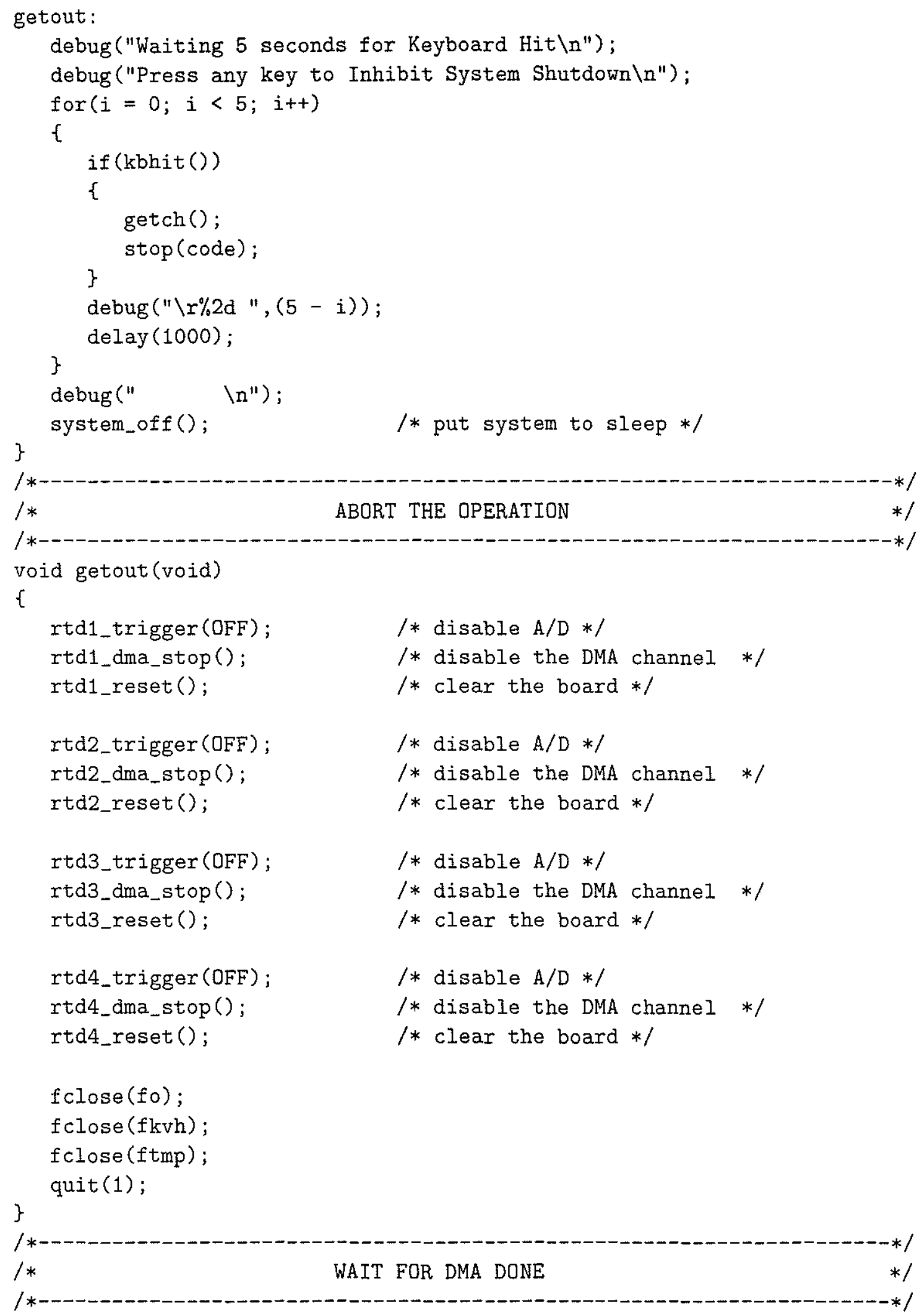




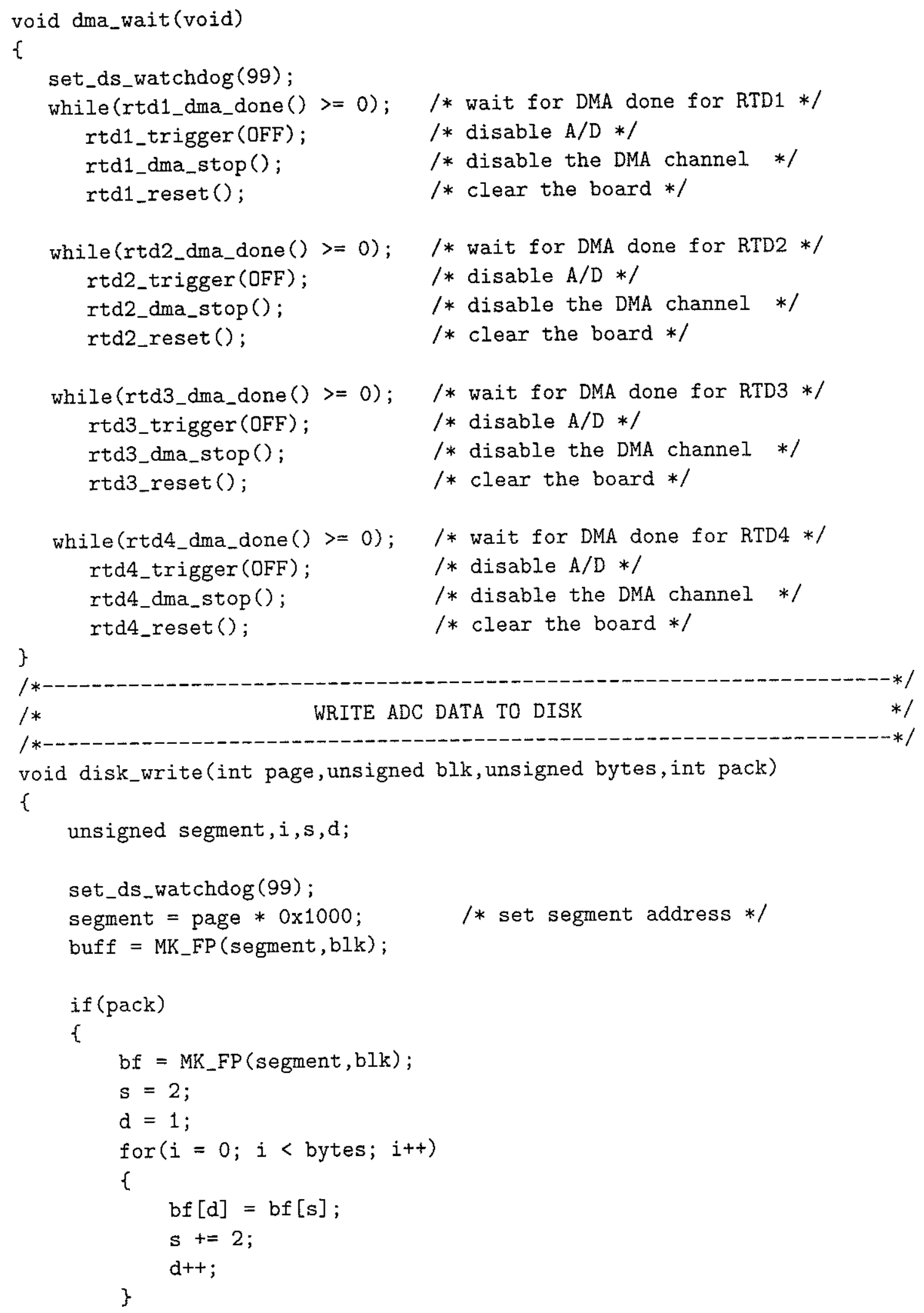




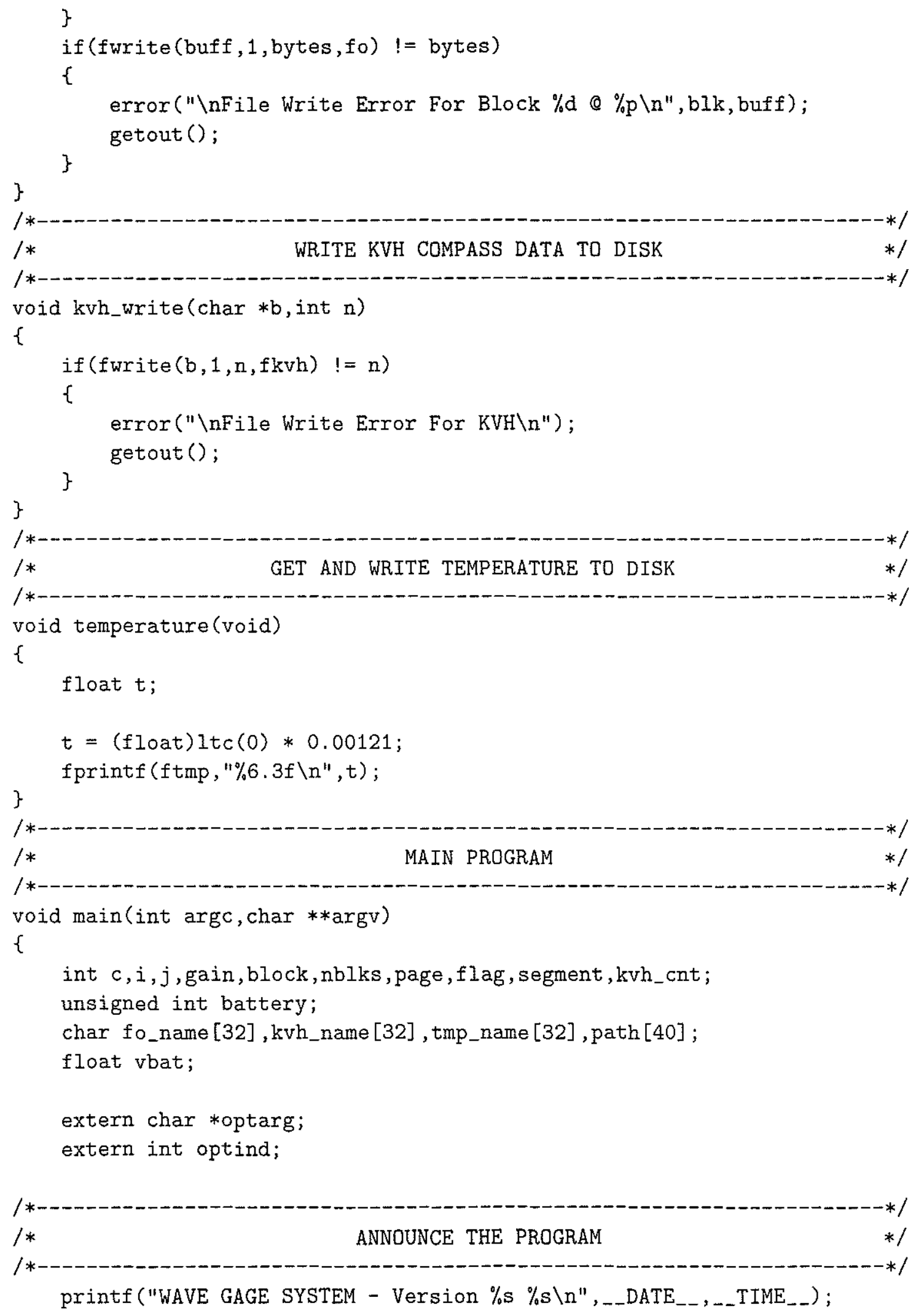




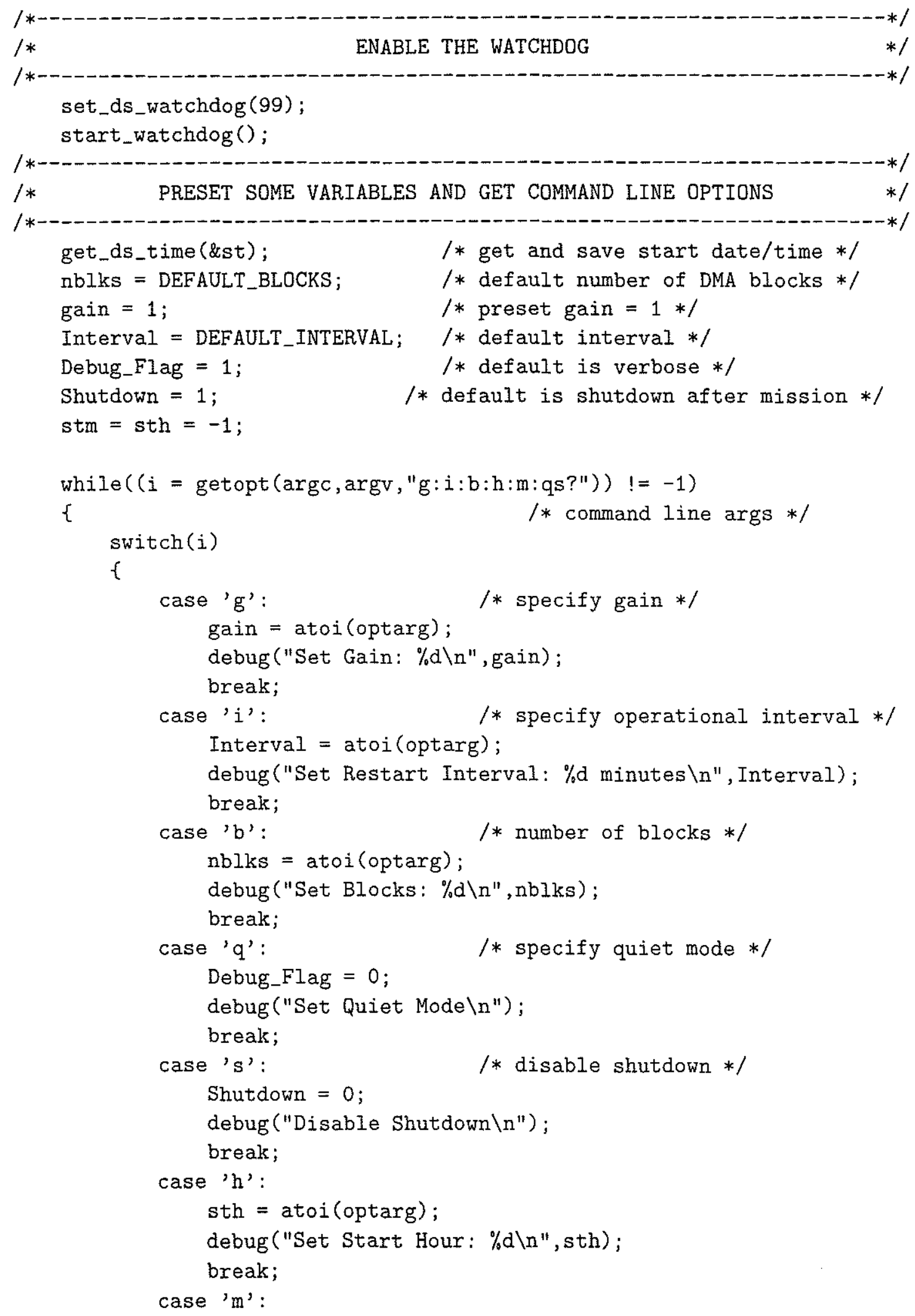




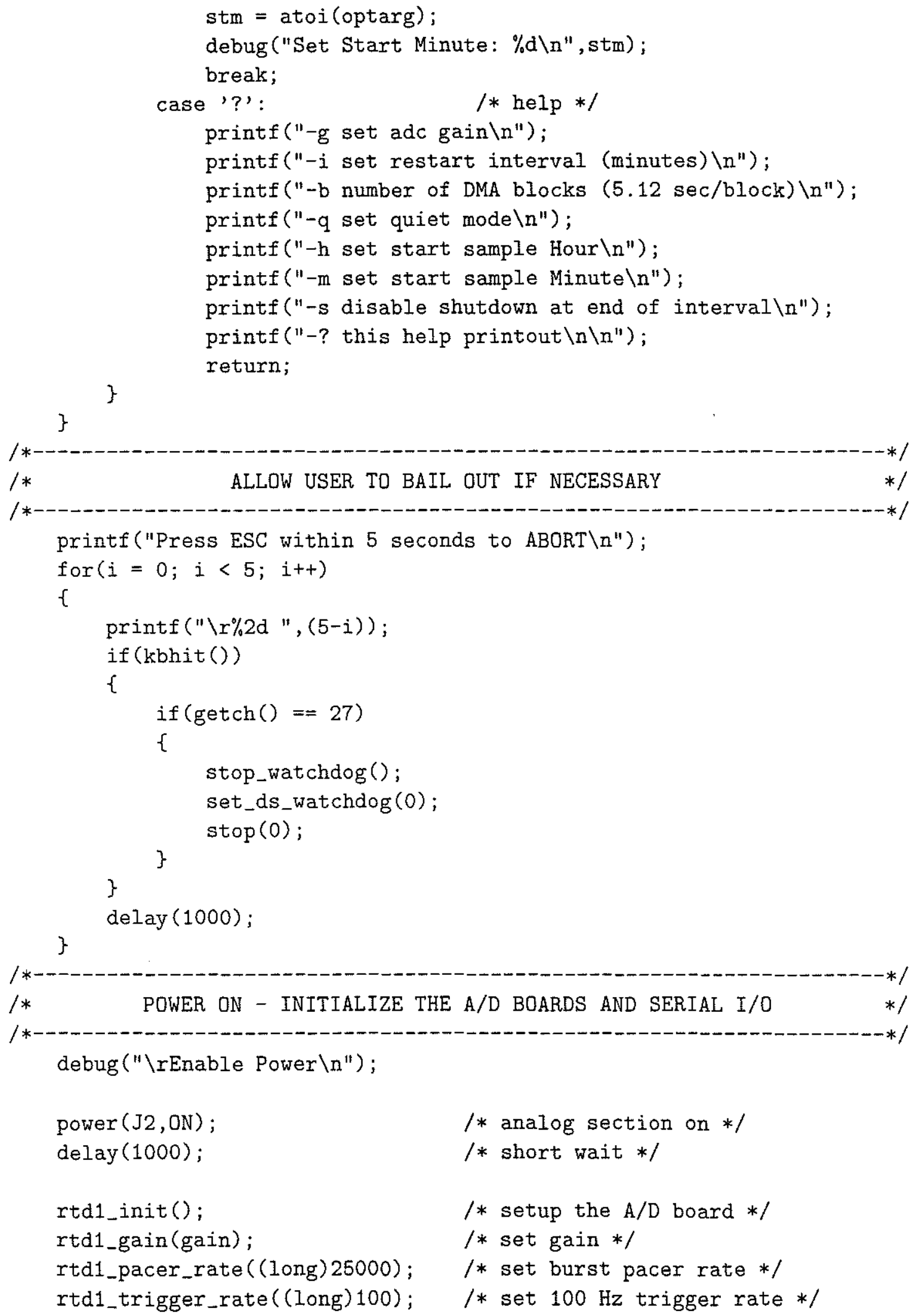


rtd2_init();

rtd2_gain (gain);

rtd2_pacer_rate ( (long) 25000);

rtd2_trigger_rate((long) 100);

rtd3_init();

rtd3_gain(gain);

rtd3_pacer_rate ( (long) 25000);

rtd3_trigger_rate ((long) 100);

rtd4_init();

rtd4_gain(gain);

rtd4_pacer_rate ((long) 25000);

rtd4_trigger_rate((long) 100);

kvh_init();
/* setup the A/D board */

$/ *$ set gain $* /$

/* set burst pacer rate */

$/ *$ set $100 \mathrm{~Hz}$ trigger rate $* /$

$/ *$ setup the $\mathrm{A} / \mathrm{D}$ board $* /$

$/ *$ set gain $* /$

/* set burst pacer rate */

$/ *$ set $100 \mathrm{~Hz}$ trigger rate $* /$

/* setup the $\mathrm{A} / \mathrm{D}$ board $* /$

$/ *$ set gain $* /$

/* set burst pacer rate $* /$

$/ *$ set $100 \mathrm{~Hz}$ trigger rate $* /$

/* init the KVH compass port */

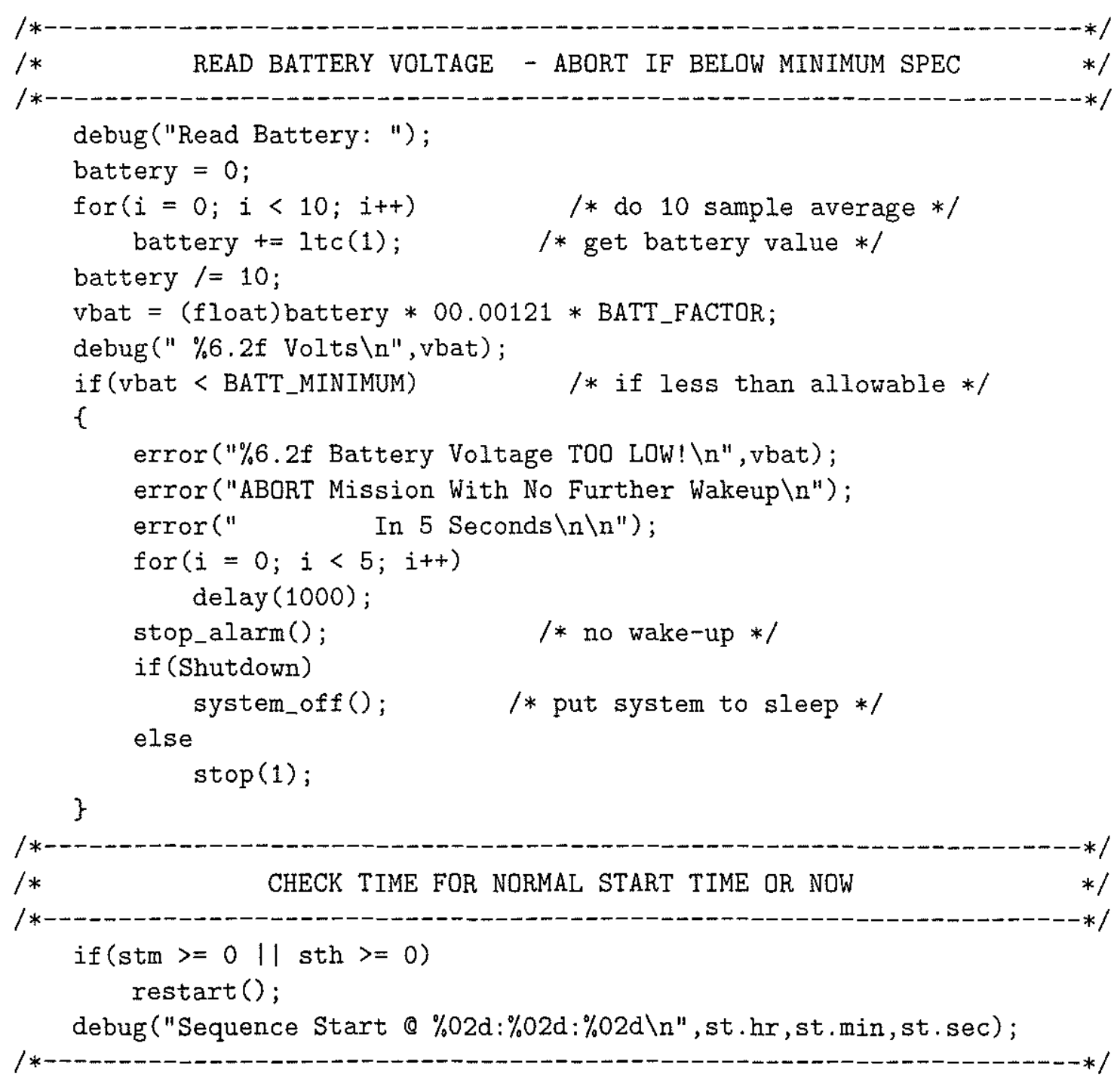




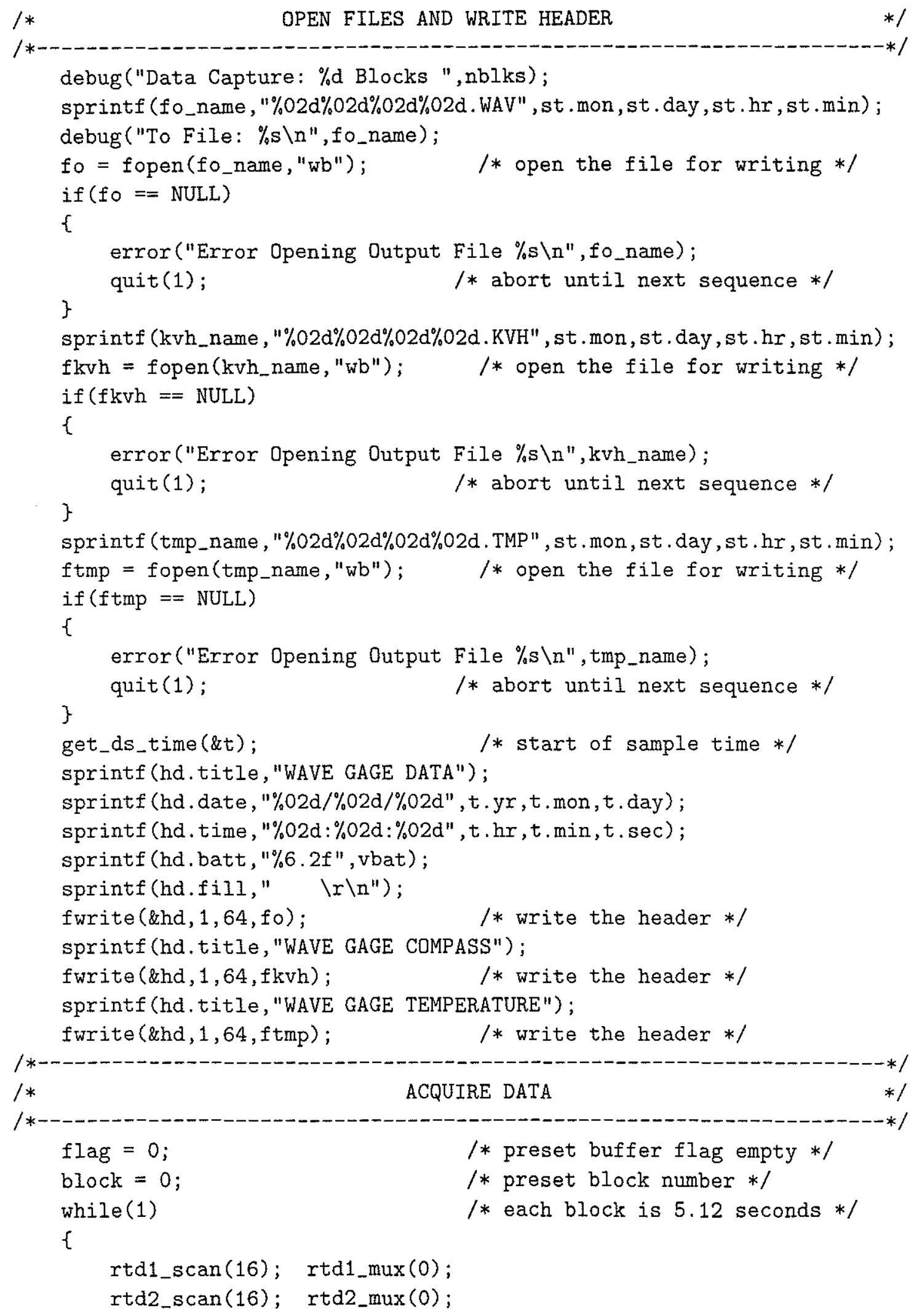




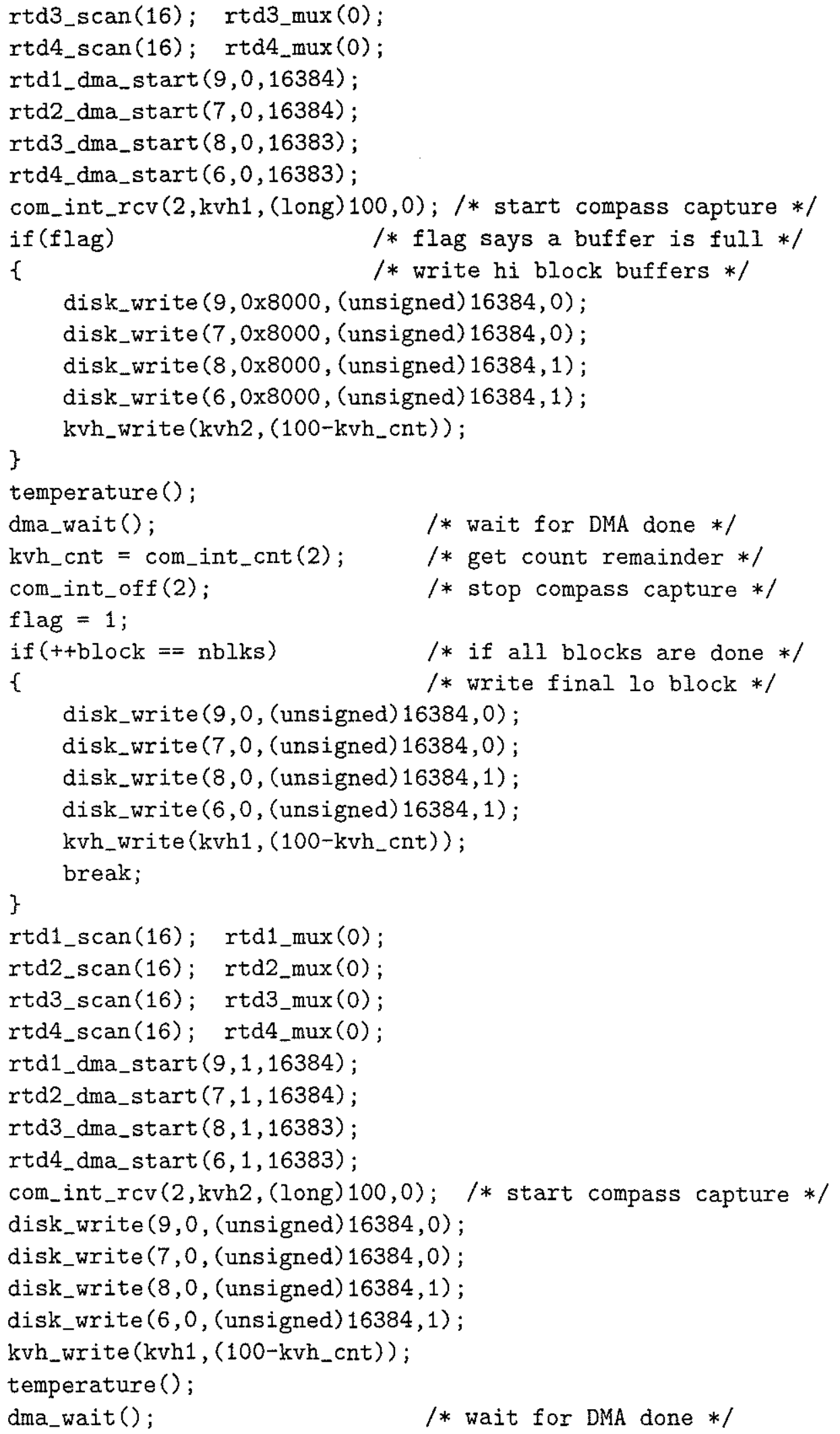




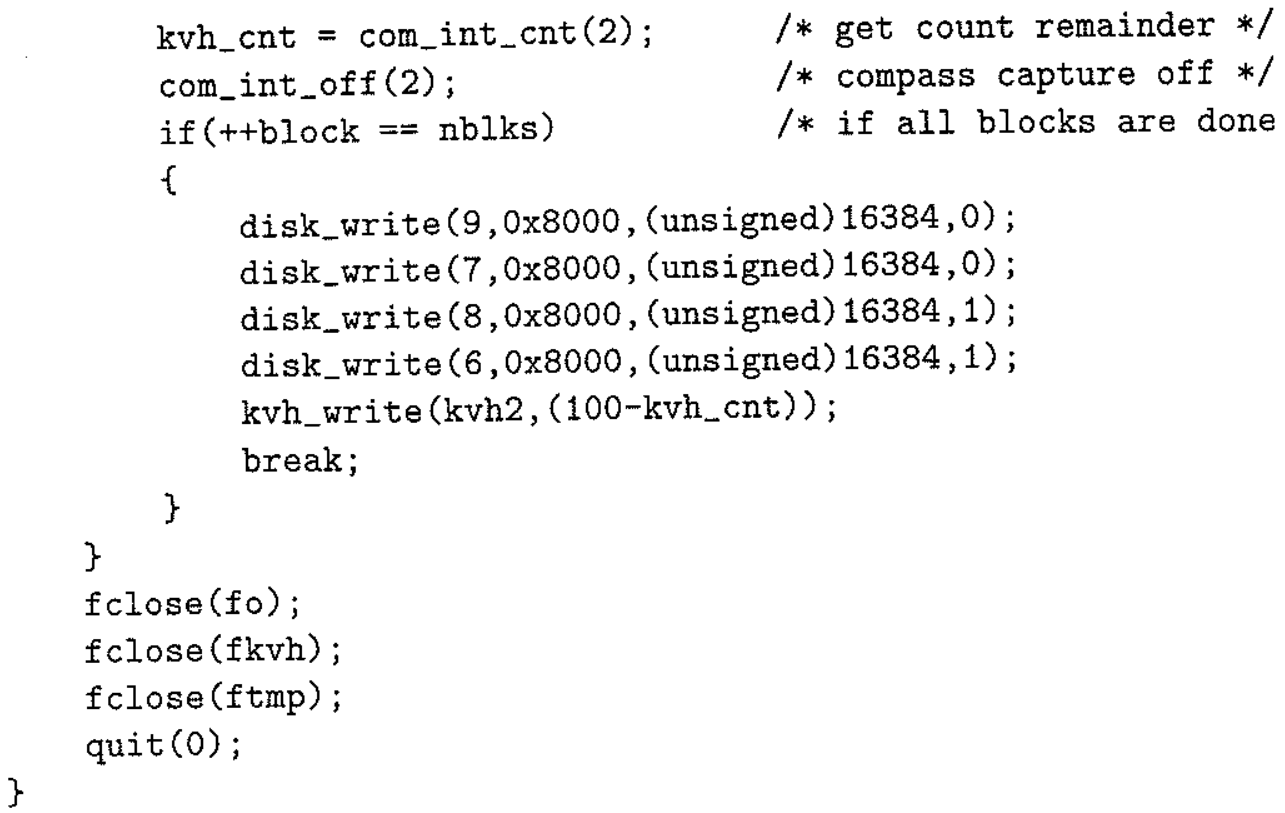

\section{H.2 Startup.c}

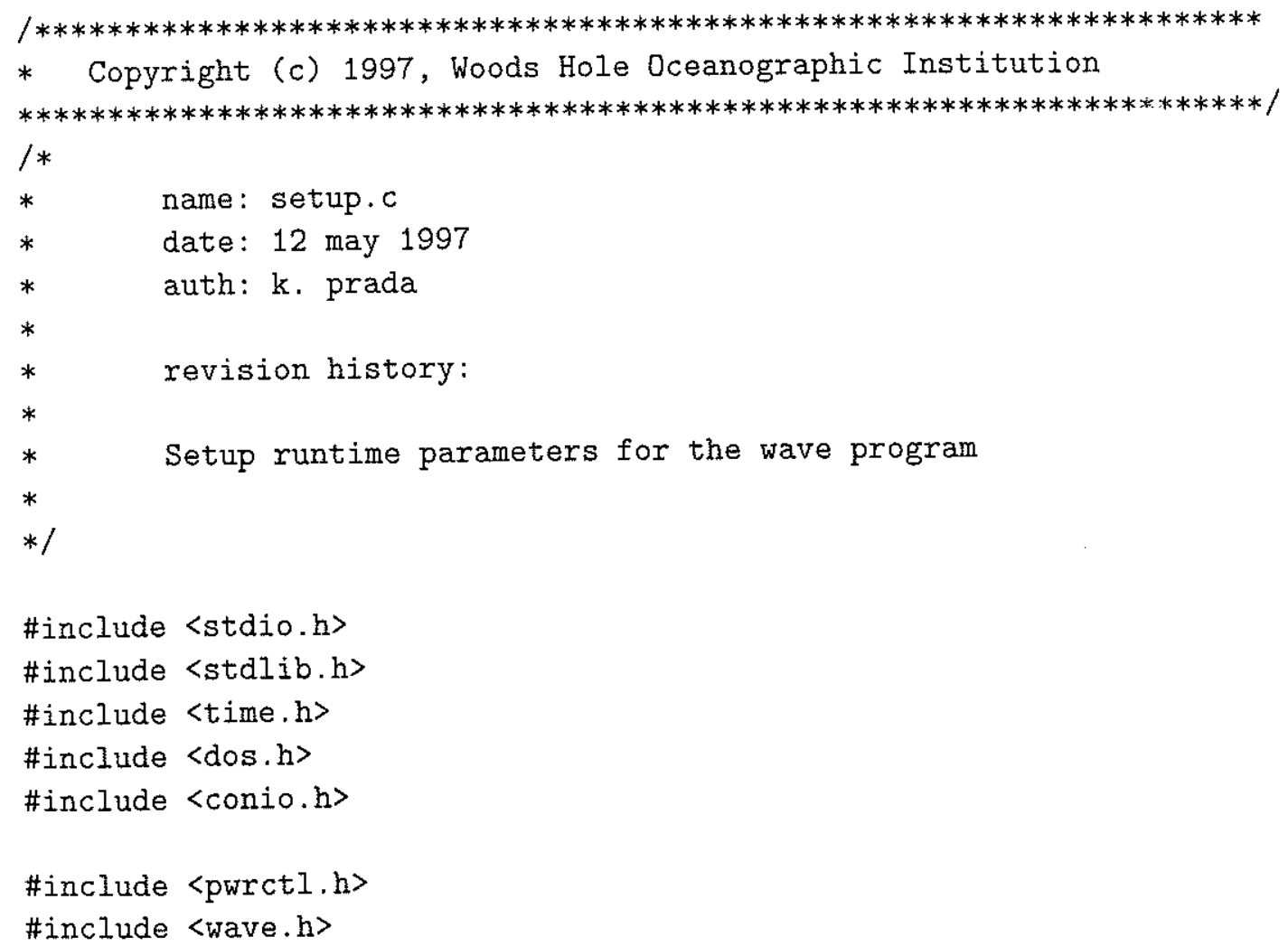




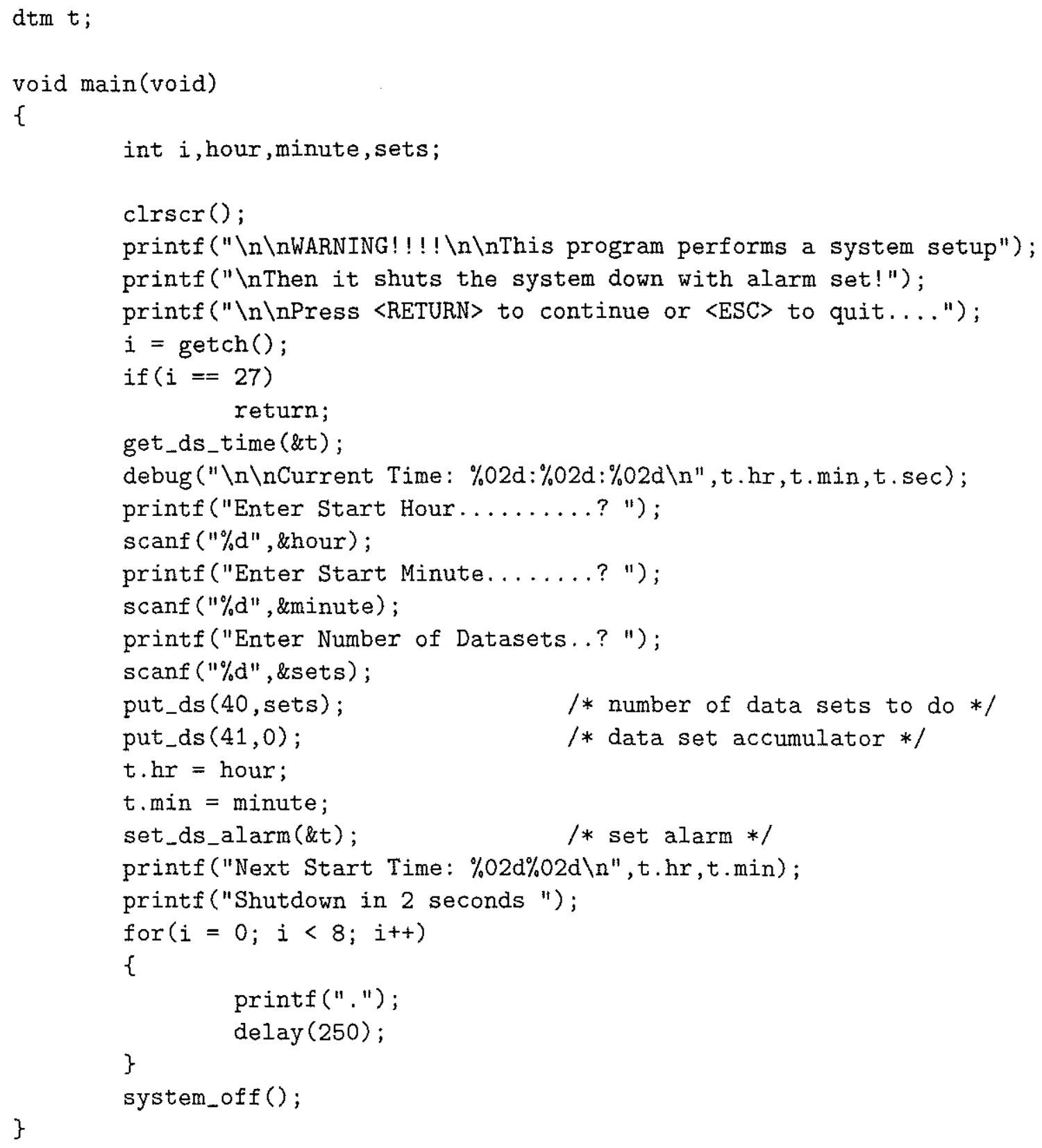




\section{Appendix I}

\section{Capacitive Wire Calibration}

The capacitive wire wave gage was calibrated using simple linear calibration curves which were generated by connecting a known capacitance to each of the electronics modules and measuring their voltage outputs the results of which are shown in Table I 1. Straight lines were fit to the calibration curves generating offsets and slopes for each wire. Additionally, when the full 63 " of wave wire 1 was submerged in saltwater, the output voltage was 835 volts. This conversion factor of length/voltage was used to generate a maximum capacitance value for each wire. It was assumed that the maximum capacitance for each wire was Identical because the wires were manufactured at the same time and were all cut to the same length. The calibration is accomplished by multiplying the measured output voltage from each capacitive wire by the calibration slope, adding the offset, and then multiplying by the conversion factor of $19.17 \mathrm{~cm} / \mathrm{uF}$.

\begin{tabular}{c|cccccc} 
Capacitance, 5\% & Wire 1 & Wire 2 & Wire 3 & Wire 4 & Wire 5 & Wire 6 \\
\hline $0(\mathrm{uF})$ & 0.02 (volts) & 0.01 & 0.02 & 0.01 & 0.01 & 0.02 \\
1 & 1.02 & 1.01 & 102 & 1.01 & 102 & 101 \\
2 & 2.02 & 2.02 & 2.03 & 2.02 & 2.04 & 2.03 \\
3 & 2.96 & 2.95 & 2.97 & 2.96 & 2.96 & 2.97 \\
4 & 4.03 & 4.02 & 4.04 & 4.03 & 4.02 & 4.04 \\
5 & 5.01 & 5.00 & 5.02 & 5.01 & 5.04 & 5.02
\end{tabular}

Table I.1: Calibration Data for Capacative Wire Wave Gage 